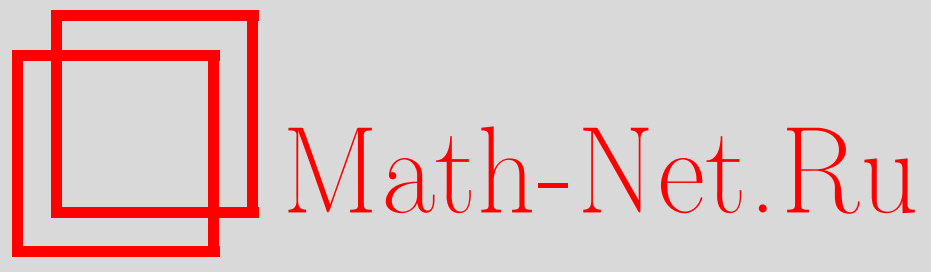

А. Б. Александров, В. В. Пеллер, Операторно липшицевы функции, УМН, 2016, том 71, выпуск 4, 3-106

DOI: https://doi.org/10.4213/rm9729

Использование Общероссийского математического портала Math-Net.Ru подразумевает, что вы прочитали и согласны с пользовательским соглашением http://www . mathnet.ru/rus/agreement

Параметры загрузки:

IP : 54.209 .52 .79

26 апреля 2023 г., $17: 36: 46$

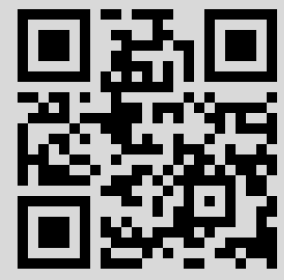




\section{Операторно липшицевы функции}

\section{А. Б. Александров, В. В. Пеллер}

Целью обзора является подробное изучение операторно липшицевых функций. Непрерывная функция $f$ на вещественной прямой $\mathbb{R}$ называется операторно липшицевой, если $\|f(A)-f(B)\| \leqslant$ const $\|A-B\|$ для любых самосопряжённых операторов $A$ и $B$. Приводятся достаточные условия и необходимые условия для операторной липшицевости. Изучается также класс операторно дифференцируемых функций на $\mathbb{R}$. Далее рассматривается класс операторно липшицевых функций на замкнутых подмножествах плоскости, а также вводится класс коммутаторно липшицевых функций на таких подмножествах. Для изучения этих классов функций важную роль играют двойные операторные интегралы и мультипликаторы Шура.

Библиография: 77 названий.

Ключевые слова: функции от операторов, операторно липшицевы функции, операторно дифференцируемые функции, самосопряжённые операторы, нормальные операторы, разделённая разность, двойные операторные интегралы, мультипликаторы Шура, дробно-линейные преобразования, классы Бесова, меры Карлесона.

DOI: $10.4213 / \mathrm{rm} 9729$

\section{СоДЕРЖАНИЕ}

1. Введение........................................ 4

2. Предварительные сведения и обозначения ................... 7

Глава I. Операторно липшицевы функции на прямой и на окружности. По

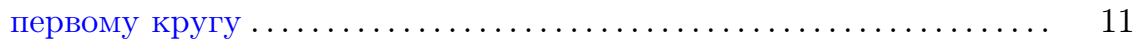

1.1. Элементарные примеры операторно липшицевых функций ......... 12

1.2. Операторная липшицевость в сравнении с операторной дифференци-

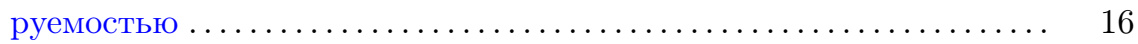

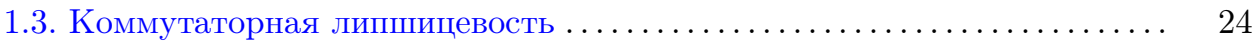

1.4. Операторные неравенства Бернштейна..................... 25

1.5. Необходимые условия для операторной липшицевости........... 27

1.6. Достаточное условие для операторной липшицевости

в терминах классов Бесова ............................ 31

1.7. Операторно гёльдеровы функции ....................... 32

Исследование первого автора выполнено при поддержке РФФИ (грант № 14-01-00198); исследование второго автора выполнено при поддержке гранта NSF DMS 130092.

(C) А. Б. АлЕксандров, В. В. ПеллеР, 2016 
1.8. Гёльдеровские функции при возмущениях операторами класса Шаттена-фон Неймана .................................... 35

Глава II. Мультипликаторы Шура и двойные операторные интегралы... 36

2.1. Дискретные мультипликаторы Шура ...................... 36

2.2. Описание дискретных мультипликаторов Шура .................. 39

2.3. Двойные операторные интегралы . . . . . . . . . . . . . . . . 43

Глава III. Операторно липшицевы функции на подмножествах плоскости 47

3.1. Операторно липшицевы и коммутаторно липшицевы функции на замкнутых подмножествах плоскости ................... 47

3.2. Ограниченные и неограниченные нормальные операторы.......... 55

3.3. Разделённые разности и коммутаторная липшицевость ........... 57

3.4. Мультипликаторы Шура и операторная липшицевость ............ 61

3.5. Роль двойных операторных интегралов .................... 63

3.6. Ядерная липшицевость и ядерно-коммутаторная липшицевость . . . . 69

3.7. Операторно липшицевы функции на плоскости. Достаточное условие 71

3.8. Достаточное условие коммутаторной липшицевости в терминах инте-

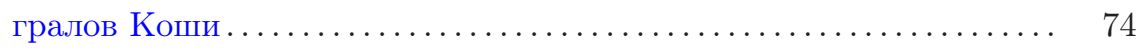

3.9. Коммутаторно липшицевы функции в круге и в полуплоскости ..... 76

3.10. Операторно липшицевы функции и дробно-линейные преобразования 79



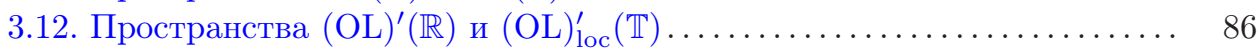

3.13. Вокруг достаточного условия Арази-Бартона-Фридмана.......... 90

3.14. В каких случаях имеет место равенство $\mathrm{OL}(\mathfrak{F})=\operatorname{Lip}(\mathfrak{F})$ ? . . . ..... 98

Заключительные замечания .............................. 99

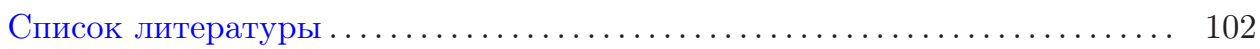

\section{1. Введение}

Одна из важнейших задач теории возмущений состоит в исследовании, насколько изменятся функции $f(A)$ от оператора $A$ при малых возмущениях оператора. В частности, естественным образом возникает задача описать класс непрерывных функций $f$ на вещественной прямой $\mathbb{R}$ таких, что справедливо неравенство

$$
\|f(A)-f(B)\| \leqslant \text { const }\|A-B\|
$$

для произвольных (ограниченных) самосопряжённых операторов $A$ и $B$ в гильбертовом пространстве. Такие функции называются операторно липшицевъми. Напомним, что функции от самосопряжённых (нормальных) операторов определяются как интегралы этих функций по спектральным мерам операторов (см. [68]).

Класс операторно липшицевых функций на $\mathbb{R}$ мы будем обозначать символом $\mathrm{OL}(\mathbb{R})$. Отметим, что если $f$ - операторно липшицева функция, то неравенство (1.1) справедливо и для неограниченных самосопряжённых операторов $A$ и $B$ с ограниченной разностью (см. теорему 3.2 .1 ниже), причём с той же самой константой. Минимальное значение этой константы является, по определению, нормой $\|f\|_{\mathrm{OL}}=\|f\|_{\mathrm{OL}(\mathbb{R})}$ функции $f$ в пространстве $\mathrm{OL}(\mathbb{R})$ (строго говоря, полунормой, которая становится нормой после отождествления функций, отличающихся на постоянную функцию). 
Ясно, что если $f$ - операторно липшицева функция, то она липшищева, т. е.

$$
|f(x)-f(y)| \leqslant \text { const }|x-y|
$$

при всех вещественных $x$ и $y$ (мы будем использовать символ $\operatorname{Lip}(\mathbb{R})$ для обозначения класса липшицевых функций на $\mathbb{R}$ ). Обратное утверждение неверно. Ю. Б. Фарфоровская в [25] построила пример липшицевой функции, не являющейся операторно липшицевой. Позже в работах [45] и [34] было установлено, что липшицева функция $x \mapsto|x|$ не операторно липшицева.

Операторно липшицевы функции играют важную роль в теории операторов и математической физике. В частности, они возникают при изучении применимости формулы следов Лифшица-Крейна:

$$
\operatorname{trace}(f(A)-f(B))=\int_{\mathbb{R}} f^{\prime}(t) \xi(t) d t
$$

(см. [41]). Здесь $A$ и $B$ - самосопряжённые операторы в гильбертовом пространстве такие, что оператор $A-B$ ядерный (т. е. $A-B \in \boldsymbol{S}_{1}$ ), а $\xi$ - функция класса $L^{1}(\mathbb{R})$ (функиия спектрального сдвига), которая определяется только операторами $A$ и $B$. Ясно, что правая часть равенства (1.2) имеет смысл для любой липшицевой функции $f$. Что касается левой части, то, как показывает пример Фарфоровской [26], условия $A-B \in \boldsymbol{S}_{1}$ и $f \in \operatorname{Lip}(\mathbb{R})$ не гарантируют того, что $f(A)-f(B) \in \boldsymbol{S}_{1}$, и, стало быть, для применимости формулы следов (1.2) для всех пар самосопряжённых операторов с ядерной разностью необходимо наложить более сильное условие на $f$. По крайней мере, функция $f$ должна обладать следующим свойством:

$$
A-B \in \boldsymbol{S}_{1} \Rightarrow f(A)-f(B) \in \boldsymbol{S}_{1}
$$

для самосопряжённых операторов $A$ и $B$. Для функций $f$ на $\mathbb{R}$ свойство (1.3) имеет место для произвольных (не обязательно ограниченных) самосопряжённых операторов в том и только том случае, если функция $f$ операторно липшицева (см. теорему 3.6.5 ниже). Оказывается (см. недавнюю работу [64]), что операторная липшицевость функции $f$ не только необходима для справедливости формулы следов (1.2) для произвольных (не обязательно ограниченных) самосопряжённых операторов $A$ и $B$ с ядерной разностью, но и достаточна.

Класс операторно липшицевых функций обладает некоторыми специфическими свойствами. Так, например, операторно липшицевы функции всюду дифференцируемы, но не обязательно непрерывно дифференцируемы (см. теорему 3.3.3 и пример 7 в разделе 1.1 ).

Оказывается, что операторная липшицевость может быть охарактеризована в терминах мультипликаторов Шура (см. раздел 3.3). Мы увидим, что непрерывная функция $f$ на $\mathbb{R}$ является операторно липшицевой тогда и только тогда, когда она всюду дифференцируема и разделённая разность $\mathfrak{D} f$,

$$
(\mathfrak{D} f)(x, y) \stackrel{\text { def }}{=} \frac{f(x)-f(y)}{x-y}, \quad x, y \in \mathbb{R},
$$

является мультипликатором Шура.

Аналогичным образом можно рассмотреть задачу для функций на окружности и унитарных операторов. Непрерывная функция $f$ на единичной окружности $\mathbb{T}$ называется операторно липшищевой, если $\|f(U)-f(V)\| \leqslant$ const $\|U-V\|$ для произвольных унитарных операторов $U$ и $V$. 
В главе I этого обзора мы обсудим необходимые условия и достаточные условия для операторной липшицевости функций на прямой $\mathbb{R}$ и окружности $\mathbb{T}$. Отметим, что в случае самосопряжённых операторов ключевую роль играет неравенство

$$
\|f(A)-f(B)\| \leqslant \text { const } \sigma\|f\|_{L^{\infty}}\|A-B\|
$$

для любых самосопряжённых операторов $A$ и $B$ с ограниченной разностью и любой ограниченной функции $f$ на $\mathbb{R}$, преобразование Фурье которой сосредоточено на $[-\sigma, \sigma], \sigma>0$. Это неравенство было получено в работах [56] и [58]. Позже в работе [10] было установлено, что неравенство (1.4) справедливо с константой 1.

По аналогии с операторно липшицевыми функциями естественно рассмотреть операторно гёльдеровы функции. Пусть $0<\alpha<1$. Функцию $f$ на $\mathbb{R}$ называют операторно гёлъдеровой порядка $\alpha$, если имеет место неравенство

$$
\|f(A)-f(B)\| \leqslant \text { const }\|A-B\|^{\alpha}
$$

для произвольных самосопряжённых операторов $A$ и $B$ в гильбертовом пространстве. Однако (см. раздел 1.7) здесь ситуация сильно отличается от случая операторно липшицевых оценок: функция $f$ является операторно гёльдеровой порядка $\alpha$ в том и только том случае, когда она входит в класс $\Lambda_{\alpha}(\mathbb{R})$ гёльдеровых функиий порядка $\alpha$, т. е. $|f(x)-f(y)| \leqslant$ const $|x-y|^{\alpha}, x, y \in \mathbb{R}$.

В главе II мы обсудим двойные операторные интегралы, т. е. выражения вида

$$
\iint \Phi(x, y) d E_{1}(x) T d E_{2}(y)
$$

Здесь $\Phi$ - ограниченная измеримая функция, $T$ - ограниченный линейный оператор в гильбертовом пространстве, а $E_{1}$ и $E_{2}$ - спектральные меры. Двойные операторные интегралы появились в работе Ю. Л. Далецкого и С. Г. Крейна [23] и подверглись систематическому изучению в работах М. Ш. Бирмана и М.3. Соломяка [19]-[21]. Уже в этих работах обнаружилось, какую важную роль двойные операторные интегралы играют в теории возмущений. Двойные операторные интегралы для произвольных ограниченных операторов $T$ определены в случае, когда функция $\Phi$ является мультипликатором Шура по отношению к $E_{1}$ и $E_{2}$. В главе II мы изучаем пространство таких мультипликаторов Шура. При этом мы сначала изучаем так называемые дискретные мультипликаторы Шура, а затем, с их помощью, - мультипликаторы Шура по отношению к спектральным мерам.

Далее, в главе III мы рассмотрим класс OL(F) операторно липшищевых функциц на произвольном замкнутом подмножестве $\mathfrak{F}$ комплексной плоскости $\mathbb{C}$, который состоит из непрерывных функций $f$ на $\mathfrak{F}$ таких, что

$$
\left\|f\left(N_{1}\right)-f\left(N_{2}\right)\right\| \leqslant \text { const }\left\|N_{1}-N_{2}\right\|
$$

для произвольных нормальных операторов $N_{1}$ и $N_{2}$, спектры которых содержатся в $\mathfrak{F}$. Мы также подробно изучаем класс коммутаторно липиицевых функций на $\mathfrak{F}$, т. е. непрерывных функций $f$ на $\mathfrak{F}$ таких, что

$$
\left\|f\left(N_{1}\right) R-R f\left(N_{2}\right)\right\| \leqslant \text { const }\left\|N_{1} R-R N_{2}\right\|
$$

для любого ограниченного оператора $R$ и произвольных нормальных операторов $N_{1}$ и $N_{2}$ со спектрами в $\mathfrak{F}$. Для изучения этих классов функций мы используем результаты главы II. 
При исследовании операторно липшицевых функций на всей плоскости, как и в случае самосопряжённых операторов, ключевую роль играет следующее обобщение неравенства (1.4):

$$
\left\|f\left(N_{1}\right)-f\left(N_{2}\right)\right\| \leqslant \text { const } \sigma\|f\|_{L^{\infty}}\left\|N_{1}-N_{2}\right\|
$$

для произвольных нормальных операторов $N_{1}$ и $N_{2}$ с ограниченной разностью и для всякой ограниченной функции $f$ на $\mathbb{R}^{2}$, преобразование Фурье которой сосредоточено в $[-\sigma, \sigma] \times[-\sigma, \sigma]$. Заметим, что доказательство неравенства (1.4), полученное в [56] и [58], не распространяется на случай нормальных операторов, и в [14] был найден новый метод получения таких оценок.

Мы также получим найденное в [3] достаточное условие для коммутаторной липшицевости функций, заданных на собственных замкнутых подмножествах плоскости, в терминах интегралов Коши мер на дополнении этого множества. С помощью этого условия мы выводим достаточное условие Арази-Бартмана-Фридмана [15] для коммутаторной липшицевости функций, аналитических в круге, а также его аналог для полуплоскости.

Наконец, мы приведём в главе III результаты, группирующиеся вокруг результатов Э. Киссина и В.С. Шульмана [39] о свойствах коммутаторно липшицевых функций на единичной окружности $\mathbb{T}$, допускающих аналитическое продолжение в единичный круг $\mathbb{D}$.

В завершающем разделе "Заключительные замечания" кратко обсуждаются результаты, не вошедшие в обзор.

Авторы выражают искреннюю благодарность В. С. Шульману за полезные замечания.

\section{2. Предварительные сведения и обозначения}

1. Классы Бесова. Пусть $w$ - бесконечно дифференцируемая функция на $\mathbb{R}$ такая, что

$$
w \geqslant 0, \quad \operatorname{supp} w \subset\left[\frac{1}{2}, 2\right], \quad \text { и } \quad w(s)=1-w\left(\frac{s}{2}\right) \quad \text { при } s \in[1,2] .
$$

Определим функции $W_{n}, n \in \mathbb{Z}$, на $\mathbb{R}^{d}$ равенством

$$
\left(\mathscr{F} W_{n}\right)(x)=w\left(\frac{\|x\|}{2^{n}}\right), \quad n \in \mathbb{Z}, \quad x=\left(x_{1}, \ldots, x_{d}\right), \quad\|x\| \stackrel{\text { def }}{=}\left(\sum_{j=1}^{d} x_{j}^{2}\right)^{1 / 2},
$$

где $\mathscr{F}$ - преобразование Фуръе, определённое на $L^{1}\left(\mathbb{R}^{d}\right)$ равенством

$$
\begin{aligned}
(\mathscr{F} f)(t) & =\int_{\mathbb{R}^{d}} f(x) e^{-\mathrm{i}(x, t)} d x, \quad x=\left(x_{1}, \ldots, x_{d}\right), \\
t & =\left(t_{1}, \ldots, t_{d}\right), \quad(x, t) \stackrel{\text { def }}{=} \sum_{j=1}^{d} x_{j} t_{j} .
\end{aligned}
$$

Ясно, что $\sum_{n \in \mathbb{Z}}\left(\mathscr{F} W_{n}\right)(t)=1, t \in \mathbb{R}^{d} \backslash\{0\}$.

Каждому медленно растущему распределению $f$ из $\mathscr{S}^{\prime}\left(\mathbb{R}^{d}\right)$ поставим в соответствие последовательность $\left\{f_{n}\right\}_{n \in \mathbb{Z}}$,

$$
f_{n} \stackrel{\text { def }}{=} f * W_{n} .
$$


Формальный ряд $\sum_{n \in \mathbb{Z}} f_{n}$, являющийся разложением типа Винера-Пэли функции $f$, не обязательно сходится к $f$. Сначала мы определим (однородный) класс Бесова $\dot{B}_{p, q}^{s}\left(\mathbb{R}^{d}\right), s \in \mathbb{R}, 0<p, q \leqslant \infty$, как пространство распределений $f$ таких, что

$$
\left\{2^{n s}\left\|f_{n}\right\|_{L^{p}}\right\}_{n \in \mathbb{Z}} \in \ell^{q}(\mathbb{Z}), \quad\|f\|_{B_{p, q}^{s}} \stackrel{\text { def }}{=}\left\|\left\{2^{n s}\left\|f_{n}\right\|_{L^{p}}\right\}_{n \in \mathbb{Z}}\right\|_{\ell^{q}(\mathbb{Z})} .
$$

В соответствии с этим определением $\dot{B}_{p, q}^{s}\left(\mathbb{R}^{d}\right)$ содержит все полиномы; при этом $\|f\|_{B_{p, q}^{s}}=0$ для всякого полинома $f$. Кроме того, распределение $f$ определено последовательностью $\left\{f_{n}\right\}_{n \in \mathbb{Z}}$ единственным образом с точностью до полинома. Легко видеть, что ряд $\sum_{n \geqslant 0} f_{n}$ сходится в $\mathscr{S}^{\prime}\left(\mathbb{R}^{d}\right)^{1}$. Однако ряд $\sum_{n<0} f_{n}$, вообще говоря, может расходиться. Можно доказать тем не менее, что ряды

$$
\sum_{n<0} \frac{\partial^{r} f_{n}}{\partial x_{1}^{r_{1}} \cdots \partial x_{d}^{r_{d}}} \quad \text { при } \quad r_{j} \geqslant 0, \quad 1 \leqslant j \leqslant d, \quad \sum_{j=1}^{d} r_{j}=r,
$$

равномерно сходятся в $\mathbb{R}^{d}$, если $r \in \mathbb{Z}_{+}$и $r>s-d / p$. Отметим, что при $q \leqslant 1$ ряды в (2.4) сходятся равномерно при более слабом условии $r \geqslant s-d / p$.

Теперь мы можем определить модифицированное (однородное) пространство Бесова $B_{p, q}^{s}\left(\mathbb{R}^{d}\right)$. Будем говорить, что $f \in B_{p, q}^{s}\left(\mathbb{R}^{d}\right)$, если выполнено условие $(2.3)$ и

$$
\frac{\partial^{r} f}{\partial x_{1}^{r_{1}} \cdots \partial x_{d}^{r_{d}}}=\sum_{n \in \mathbb{Z}} \frac{\partial^{r} f_{n}}{\partial x_{1}^{r_{1}} \cdots \partial x_{d}^{r_{d}}} \quad \text { при } \quad r_{j} \geqslant 0, \quad 1 \leqslant j \leqslant d, \quad \sum_{j=1}^{d} r_{j}=r,
$$

в пространстве $\mathscr{S}^{\prime}\left(\mathbb{R}^{d}\right)$, где $r$ - минимальное неотрицательное целое число такое, что $r>s-d / p(r \geqslant s-d / p$, если $q \leqslant 1)$. Теперь функция $f$ однозначно определяется последовательностью $\left\{f_{n}\right\}_{n \in \mathbb{Z}}$ с точностью до полинома степени меньше $r$. При этом полином $g$ входит в $B_{p, q}^{s}\left(\mathbb{R}^{d}\right)$ тогда и только тогда, когда $\operatorname{deg} g<r$.

В случае $p=q$ мы используем обозначение $B_{p}^{s}\left(\mathbb{R}^{d}\right)$ вместо $B_{p, p}^{s}\left(\mathbb{R}^{d}\right)$.

Рассмотрим теперь шкалу $\Lambda_{\alpha}\left(\mathbb{R}^{d}\right), \alpha>0$, классов Гёльдера-Зигмунда. Их можно определить равенством $\Lambda_{\alpha}\left(\mathbb{R}^{d}\right) \stackrel{\text { def }}{=} B_{\infty}^{\alpha}\left(\mathbb{R}^{d}\right)$.

Классы Бесова допускают много других описаний. Приведём описание в терминах конечных разностей. Для $h$ из $\mathbb{R}^{d}$ определим разностный оператор $\Delta_{h}$ равенством $\left(\Delta_{h} f\right)(x)=f(x+h)-f(x), x \in \mathbb{R}^{d}$.

Пусть $s>0, m \in \mathbb{Z}$ и $m-1 \leqslant s<m$. Пусть $p, q \in[1,+\infty]$. Класс Бесова $B_{p, q}^{s}\left(\mathbb{R}^{d}\right)$ можно определить как множество функций $f$ из $L_{\mathrm{loc}}^{1}\left(\mathbb{R}^{d}\right)$ таких, что

$$
\int_{\mathbb{R}^{d}}|h|^{-d-s q}\left\|\Delta_{h}^{m} f\right\|_{L^{p}}^{q} d h<\infty, \quad q<\infty ; \quad \sup _{h \neq 0} \frac{\left\|\Delta_{h}^{m} f\right\|_{L^{p}}}{|h|^{s}}<\infty, \quad q=\infty .
$$

Однако при этом определении классы Бесова могут содержать полиномы более высоких степеней, чем в случае определения в терминах свёрток с функциями $W_{n}$.

\footnotetext{
13десь и далее считаем, что в $\mathscr{S}^{\prime}\left(\mathbb{R}^{d}\right)$ введена слабая топология $\sigma\left(\mathscr{S}^{\prime}\left(\mathbb{R}^{d}\right), \mathscr{S}\left(\mathbb{R}^{d}\right)\right)$.
} 
Пространство $B_{p, q}^{s}$ может быть определено в терминах интеграла Пуассона. Пусть $P_{d}(x, t)$ - ядро Пуассона на $\mathbb{R}_{+}^{d+1} \stackrel{\text { def }}{=}\left\{(x, t): x \in \mathbb{R}^{d}, t>0\right\}$, т. е. $P_{d}(x, t)=$ $c_{d} t\left(|x|^{2}+t^{2}\right)^{-(d+1) / 2}, c_{d}=\pi^{-(d+1) / 2} \Gamma((d+1) / 2)$. С каждой функцией $f$ из $L^{1}\left(\mathbb{R}^{d},(\|x\|+1)^{-(d+1)} d x\right)$ можно связать интеграл Пуассона $\mathcal{P} f$ :

$$
(\mathcal{P} f)(x, t)=\int_{\mathbb{R}^{d}} P_{d}(x-y, t) f(y) d y .
$$

Тогда для любого целого положительного $m$ имеет место равенство

$$
\frac{\partial^{m}(\mathcal{P} f)}{\partial t^{m}}(x, t)=\int_{\mathbb{R}^{d}} \frac{\partial^{m} P_{d}(x-y, t)}{\partial t^{m}} f(y) d y .
$$

Заметим, что интеграл в (2.5) имеет смысл для всех функций $f$, принадлежащих пространству $L^{1}\left(\mathbb{R}^{d},(\|x\|+1)^{-(d+m+1)} d x\right)$, что позволяет корректно определить $\frac{\partial^{m}}{\partial t^{m}} \mathcal{P} f$.

Пусть $m \in \mathbb{Z}, m-1 \leqslant s<m, 1 \leqslant p, q \leqslant+\infty$. Мы можем определить $B_{p, q}^{s}$ как множество всех функций $f \in L^{1}\left(\mathbb{R}^{d},(\|x\|+1)^{-(d+m+1)} d x\right)$ таких, что

$$
\begin{aligned}
\left(\int_{0}^{\infty} t^{(m-s) q-1}\left\|\left(\frac{\partial^{m}}{\partial t^{m}} \mathcal{P} f\right)(\cdot, t)\right\|_{L^{p}\left(\mathbb{R}^{d}\right)}^{q} d t\right)^{1 / q}<+\infty, & q<+\infty, \\
\sup _{t>0} t^{m-s}\left\|\left(\frac{\partial^{m}}{\partial t^{m}} \mathcal{P} f\right)(\cdot, t)\right\|_{L^{p}\left(\mathbb{R}^{d}\right)}<+\infty, & q=+\infty .
\end{aligned}
$$

При этом определении классы Бесова тоже могут содержать полиномы более высоких степеней, чем в случае определения в терминах свёрток с функциями $W_{n}$. Отметим ещё, что это определение в терминах интеграла Пуассона при определённых условиях и оговорках работает и в том случае, когда $p<1$ или $q<1$.

Перейдём теперь к классам Бесова функций на единичной окружности $\mathbb{T}$. Пусть $w$ - функция, удовлетворяющая условиям (2.1). Определим тригонометрические полиномы $W_{n}, n \geqslant 0$, равенством

$$
W_{n}(\zeta) \stackrel{\text { def }}{=} \sum_{j \in \mathbb{Z}} w\left(\frac{|j|}{2^{n}}\right) \zeta^{j}, \quad n \geqslant 1, \quad W_{0}(\zeta) \stackrel{\text { def }}{=} \sum_{\{j:|j| \leqslant 1\}} \zeta^{j}, \quad \zeta \in \mathbb{T} .
$$

Если $f$ - распределение на $\mathbb{T}$, положим $f_{n}=f * W_{n}, n \geqslant 0$, и будем говорить, что $f$ входит в класс Бесова $B_{p, q}^{s}(\mathbb{T}), s \in \mathbb{R}, 0<p, q \leqslant \infty$, если

$$
\left\{2^{n s}\left\|f_{n}\right\|_{L^{p}}\right\}_{n \geqslant 0} \in \ell^{q} .
$$

Пусть $s \in \mathbb{R}, s>\max \{0,1 / p-1\}, m$ - целое положительное число такое, что $m>\max \{s, s+1 / p-1\}$. Тогда обобщённая функция $f$ на окружности $\mathbb{T}$ принадлежит пространству $B_{p, q}^{s}(\mathbb{T})$ в том и только том случае, когда

$$
\begin{array}{r}
\int_{0}^{1} r\left(1-r^{2}\right)^{(m-s) q-1}\left\|\frac{\partial^{m}}{\partial r^{m}}((\mathcal{P} f)(r \zeta))\right\|_{L^{p}(\mathbb{T})}^{q} d r<+\infty, \quad q<+\infty, \\
\sup _{r \in[0,1)}\left(1-r^{2}\right)^{m-s}\left\|\frac{\partial^{m}}{\partial r^{m}}((\mathcal{P} f)(r \zeta))\right\|_{L^{p}(\mathbb{T})}<+\infty, \quad q=+\infty,
\end{array}
$$

где $\mathcal{P} f$ обозначает интеграл Пуассона обобщённой функции $f$. 
В определениях классов Бесова в терминах интеграла Пуассона мы рассматривали производную порядка $m$ по переменной $t$ в первом случае и по переменной $r$ - во втором. Хорошо известно, что в обоих случаях мы получили бы равносильное определение, если бы потребовали конечность аналогичных выражений для всевозможных (в том числе и смешанных) частных производных порядка $m$.

Более подробную информацию о классах Бесова можно найти в [52] и [74].

2. Классы Шаттена-фон Неймана. Для ограниченного линейного оператора $T$ в гильбертовом пространстве его сингулярные числа $s_{j}(T), j \geqslant 0$, определяются равенством

$$
s_{j}(T) \stackrel{\text { def }}{=} \inf \{\|T-R\|: \operatorname{rank} R \leqslant j\} .
$$

Класс Шаттена-фон Неймана $\boldsymbol{S}_{p}, 0<p<\infty$, состоит по определению из операторов $T$, для которых

$$
\|T\|_{S_{p}} \stackrel{\text { def }}{=}\left(\sum_{j \geqslant 0}\left(s_{j}(T)\right)^{p}\right)^{1 / p}<\infty .
$$

При $p \geqslant 1$ это - нормированный идеал операторов в гильбертовом пространстве. Класс $\boldsymbol{S}_{1}$ называется классом ядерных операторов. Если $T$ - ядерный оператор в гильбертовом пространстве $\mathscr{H}$, то его след $\operatorname{trace} T$ определяется равенством trace $T \stackrel{\text { def }}{=} \sum_{j \geqslant 0}\left(T e_{j}, e_{j}\right)$, где $\left\{e_{j}\right\}_{j \geqslant 0}-$ ортонормированный базис в $\mathscr{H}$. Правая часть не зависит от выбора базиса.

Класс $\boldsymbol{S}_{2}$ называется классом Гилъберта-Шмидта. Он образует гильбертово пространство со скалярным произведением $(T, R)_{\boldsymbol{S}_{2}} \stackrel{\text { def }}{=} \operatorname{trace}\left(T R^{*}\right)$.

При $p \in(1, \infty)$ двойственное пространство $\left(\boldsymbol{S}_{p}\right)^{*}$ можно изометрическим образом отождествить с пространством $\boldsymbol{S}_{p^{\prime}}, 1 / p+1 / p^{\prime}=1$, с помощью билинейной формы $\langle T, R\rangle \stackrel{\text { def }}{=} \operatorname{trace}(T R)$. Пространство, сопряжённое с $\boldsymbol{S}_{1}$, можно отождествить с пространством ограниченных линейных операторов с помощью этой же билинейной формы, в то время как пространство, сопряжённое с пространством компактных операторов, отождествляется с $\boldsymbol{S}_{1}$.

Отсылаем читателя к [28] за более подробной информацией.

3. Операторы Ганкеля. Для функции $\varphi$ класса $L^{2}$ на единичной окружности $\mathbb{T}$ оператор Ганкеля $H_{\varphi}$ определяется на плотном в пространстве Харди $H^{2}$ множестве полиномов равенством $H_{\varphi} f \stackrel{\text { def }}{=} \mathbb{P}_{-} \varphi f$, где $\mathbb{P}_{-}$- ортогональный проектор из $L^{2}$ на $H_{-}^{2} \stackrel{\text { def }}{=} L^{2} \ominus H^{2}$. По теореме Нехари оператор $H_{\varphi}$ продолжается до ограниченного оператора из $H^{2}$ в $H_{-}^{2}$ тогда и только тогда, когда существует функция $\psi$ класса $L^{\infty}$ на $\mathbb{T}$, коэффициенты Фурье $\widehat{\psi}(n)$ которой удовлетворяют равенству $\widehat{\psi}(n)=\widehat{\varphi}(n)$ при $n<0$. В свою очередь, по теореме Ч. Феффермана последнее свойство эквивалентно тому, что $\mathbb{P}_{-} \varphi$ входит в класс ВМО.

Оператор Ганкеля $H_{\varphi}$ входит в класс Шаттена-фон Неймана $\boldsymbol{S}_{p}$ в том и только том случае, когда функция $\mathbb{P}_{-} \varphi$ входит в класс Бесова $B_{p}^{1 / p}(\mathbb{T})$. При $p \geqslant 1$ это было доказано в работе [54], а при $p \in(0,1)$ - в [55] (см. также [53] и [69], где даны другие доказательства при $p<1)$. 
Легко видеть, что оператор $H_{\varphi}$ имеет в базисах $\left\{z^{j}\right\}_{j \geqslant 0}$ и $\left\{\bar{z}^{k}\right\}_{k \geqslant 1}$ матрицу $\{\widehat{\varphi}(-j-k-1)\}_{j \geqslant 0, k>1}$. Такие матрицы, т. е. матрицы вида $\left\{\alpha_{j+k}\right\}_{j, k \geqslant 0}$, называются ганкелевыми. Критерий принадлежности операторов Ганкеля классу $\boldsymbol{S}_{p}$ может быть переформулирован так: оператор в пространстве $\ell^{2}$ с ганкелевой матрицей $\left\{\alpha_{j+k}\right\}_{j, k \geqslant 0}$ входит в класс $\boldsymbol{S}_{p}, p>0$, в том и только том случае, когда функция $\sum_{j \geqslant 0} \alpha_{j} z^{j}$ входит в класс Бесова $B_{p}^{1 / p}(\mathbb{T})$.

Мы отсылаем читателя к монографии [60] за доказательствами перечисленных выше результатов и более подробной информацией об операторах Ганкеля.

4. Обозначения. Приведём список некоторых обозначений, используемых в обзоре:

$\mathrm{OL}(\mathfrak{F})$ - пространство операторно липшицевых функций на замкнутом подмножестве $\mathfrak{F}$ комплексной плоскости $\mathbb{C}$;

$\mathrm{CL}(\mathfrak{F})$ - пространство коммутаторно липшицевых функций на замкнутом подмножестве $\mathfrak{F}$ комплексной плоскости $\mathbb{C}$;

$\mathrm{OD}(\mathbb{R})$ - пространство операторно дифференцируемых функций на $\mathbb{R} ;$

$\mathcal{B}\left(\mathscr{H}_{1}, \mathscr{H}_{2}\right)$ - пространство ограниченных линейных операторов из гильбертова пространства $\mathscr{H}_{1}$ в гильбертово пространство $\mathscr{H}_{2}, \mathcal{B}(\mathscr{H}) \stackrel{\text { def }}{=} \mathcal{B}(\mathscr{H}, \mathscr{H})$;

$\mathcal{B}_{\mathrm{sa}}(\mathscr{H})$ - пространство ограниченных самосопряжённых операторов в гильбертовом пространстве $\mathscr{H}$;

$\boldsymbol{m}$ - нормированная мера Лебега на единичной окружности $\mathbb{T}$;

$\boldsymbol{m}_{2}$ - мера Лебега на плоскости.

\section{Глава I. Операторно липшицевы функции на прямой и на окружности. По первому кругу}

В этой вводной главе мы рассмотрим операторно липшицевы функции на вещественной прямой $\mathbb{R}$ и на единичной окружности $\mathbb{T}$. Позже, в главе III, мы подвергнем класс операторно липшицевых функций более подробному изучению, а также перейдём к изучению операторно липшицевых функций на замкнутых подмножествах комплексной плоскости $\mathbb{C}$.

Мы используем обозначение $\mathrm{OL}(\mathbb{R})$ для класса операторно липшицевых функций на $\mathbb{R}$; для функции $f$ из $\mathrm{OL}(\mathbb{R})$ положим

$\|f\|_{\mathrm{OL}(\mathbb{R})} \stackrel{\text { def }}{=} \sup \left\{\frac{\|f(A)-f(B)\|}{\|A-B\|}: A, B-\right.$ самосопряжённые операторы, $\left.A \neq B\right\}$.

Аналогичным образом вводится пространство OL(T) операторно липшицевых функций на $\mathbb{T}$, при этом самосопряжённые операторы заменяются на унитарные.

Оказывается, что класс $\mathrm{OL}(\mathbb{R})$ имеет несколько необычные свойства. В частности, функции из этого класса должны быть дифференцируемы всюду на $\mathbb{R}$, а также должны иметь производную на бесконечности, т. е. должен существовать предел $\lim _{|t| \rightarrow \infty} f(t) / t$ (см. теорему 3.3.3 ниже). Заметим, что отсюда вытекает упомянутый во введении результат Макинтоша-Като: функция $x \mapsto|x|$ не является операторно липшицевой. С другой стороны, функции класса $\mathrm{OL}(\mathbb{R})$ не обязаны быть непрерывно дифференцируемыми. В частности, 
функция $x \mapsto x^{2} \sin (1 / x)$, не будучи непрерывно дифференцируемой, является операторно липшицевой (см. теорему 1.1.4 ниже).

Эту главу мы начнём с элементарных примеров операторно липшицевых функций (см. раздел 1.1).

Мы введём в разделе 1.2 класс операторно дифференцируемых функций и класс локально операторно дифференцируемых функций. Оказывается, что при определении этих классов неважно, рассматривать дифференцируемость в смысле Гато или в смысле Фреше. Мы увидим, что (локально) операторно дифференцируемые функции должны быть непрерывно дифференцируемыми и что операторно дифференцируемые функции обязательно должны быть операторно липшицевыми. Однако не всякая операторно липшицева функция операторно дифференцируема.

Помимо операторно липшицевых функций мы рассмотрим в разделе 1.3 коммутаторно липшицевы функции, т. е. функции $f$ на $\mathbb{R}$ такие, что

$$
\|f(A) R-R f(B)\| \leqslant \text { const }\|A R-R B\|
$$

для любых самосопряжённых операторов $A$ и $B$ (снова неважно, ограниченных или не обязательно ограниченных) и для любого ограниченного оператора $R$. Коммутаторно липшицева норма $\|f\|_{\mathrm{CL}(\mathbb{R})}$ функции $f$ определяется как минимальная константа, при которой неравенство справедливо. Аналогичным образом можно определить коммутаторно липшицевы функции на единичной окружности, если мы заменим самосопряжённые операторы на унитарные.

Оказывается, что для функций на прямой (равно как и для функций на окружности) класс коммутаторно липшицевых функций совпадает с классом операторно липиицевых функций. В главе III мы увидим, что для функций на произвольном замкнутом подмножестве плоскости $\mathbb{R}^{2}$ это уже не так.

Мы получим в этой главе достаточное условие для операторной липшицевости на прямой и на окружности (см. раздел 1.6), равно как и необходимое (см. раздел 1.5), и сравним их друг с другом.

Было бы также естественно рассмотреть класс операторно гёльдеровых функицй порядка $\alpha, 0<\alpha<1$, т. е. класс функций $f$ таких, что

$$
\|f(A)-f(B)\| \leqslant \text { const }\|A-B\|^{\alpha}
$$

для самосопряжённых операторов $A$ и $B$ в гильбертовом пространстве. Однако этот термин оказывается коротко живущим, ибо всякая функщия $f$ на $\mathbb{R}$ класса Гёлъдера порядка а с необходимостью является операторно гёлъдеровой порядка $\alpha$ (см. раздел 1.7).

\section{1. Элементарные примеры операторно липшицевых функций}

В этом разделе мы приведём примеры операторно липшицевых функций на прямой и простые достаточные условия для операторной липшицевости.

Пример 1. При всех $\lambda$ из $\mathbb{C} \backslash \mathbb{R}$ функция $(\lambda-x)^{-1}$ является операторно липшицевой на $\mathbb{R}$ и $\left\|(\lambda-x)^{-1}\right\|_{\mathrm{OL}(\mathbb{R})}=|\operatorname{Im} \lambda|^{-2}$.

ДокАзАтельство. Из резольвентного тождества Гильберта

$$
(\lambda I-A)^{-1}-(\lambda I-B)^{-1}=(\lambda I-A)^{-1}(A-B)(\lambda I-B)^{-1}
$$


мгновенно вытекает, что $\left\|(\lambda-x)^{-1}\right\|_{\mathrm{OL}(\mathbb{R})} \leqslant|\operatorname{Im} \lambda|^{-2}$. Остаётся заметить, что $\left\|(\lambda-x)^{-1}\right\|_{\mathrm{OL}(\mathbb{R})} \geqslant\left\|(\lambda-x)^{-1}\right\|_{\operatorname{Lip}(\mathbb{R})}=|\operatorname{Im} \lambda|^{-2}$.

Пример $1^{\prime}$. При всех $\lambda$ из $\mathbb{C} \backslash \mathbb{T}$ функция $(\lambda-z)^{-1}$ является операторно липшицевой на $\mathbb{T}$ и $\left\|(\lambda-z)^{-1}\right\|_{\mathrm{OL}(\mathbb{T})}=(|\lambda|-1)^{-2}$.

ПримеР 2. Функция $x \mapsto \log (1+\mathrm{i} x)$ является операторно липшицевой на $\mathbb{R}$ и $\|\log (1+\mathrm{i} x)\|_{\mathrm{OL}(\mathbb{R})}=1$. Здесь $\log$ обозначает главную ветвь логарифма. этому

ДокаЗАтельство. Ясно, что $\log (1+\mathrm{i} x)=\int_{0}^{+\infty}\left(\frac{1}{1+t}-\frac{1}{1+t+\mathrm{i} x}\right) d t$. По-

$$
\begin{aligned}
\|\log (1+\mathrm{i} x)\|_{\mathrm{OL}(\mathbb{R})} & \leqslant \int_{0}^{+\infty}\left\|\frac{1}{1+t}-\frac{1}{1+t+\mathrm{i} x}\right\|_{\mathrm{OL}(\mathbb{R})} d t \\
& =\int_{0}^{+\infty}\left\|\frac{1}{1+t+\mathrm{i} x}\right\|_{\mathrm{OL}(\mathbb{R})} d t=\int_{0}^{+\infty} \frac{d t}{(1+t)^{2}}=1 .
\end{aligned}
$$

C другой стороны, неравенство $\|\log (1+\mathrm{i} x)\|_{\mathrm{OL}(\mathbb{R})} \geqslant 1$ очевидно, поскольку $\|\log (1+\mathrm{i} x)\|_{\mathrm{OL}(\mathbb{R})} \geqslant\|\log (1+\mathrm{i} x)\|_{\operatorname{Lip}(\mathbb{R})}=1$.

Аналогичным образом можно доказать, что при всех $\lambda$ из $\mathbb{C} \backslash \mathbb{R}$ имеет место равенство $\|\log (\lambda-x)\|_{\mathrm{OL}(\mathbb{R})}=|\operatorname{Im} \lambda|^{-1}$, где $\log (\lambda-x)$ обозначает любую регулярную на $\mathbb{R}$ ветвь функции $\log (\lambda-z)$.

ПримеР 3. Функция $\operatorname{arctg}$ операторно липшицева и $\|\operatorname{arctg}\|_{\mathrm{OL}(\mathbb{R})}=1$.

ДокАзАтельство. Достаточно проверить, что $\|\operatorname{arctg}\|_{\mathrm{OL}(\mathbb{R})} \leqslant 1$. Для этого заметим, что $\operatorname{arctg} x=\operatorname{Im} \log (1+\mathrm{i} x), x \in \mathbb{R}$.

ПримеР 4. При любом натуральном $n$ верно равенство $\left\|(\lambda-x)^{-n}\right\|_{\mathrm{OL}(\mathbb{R})}=$ $n|\operatorname{Im} \lambda|^{-n-1}$ для всех $\lambda \in \mathbb{C} \backslash \mathbb{R}$.

ДокАЗАТЕЛЬСтво. Подставляя $X=(\lambda I-A)^{-1}$ и $Y=(\lambda I-B)^{-1}$ в элементарное тождество

получаем

$$
X^{n}-Y^{n}=\sum_{k=1}^{n} X^{n-k}(X-Y) Y^{k-1}
$$

$$
(\lambda I-A)^{-n}-(\lambda I-B)^{-n}=\sum_{k=1}^{n}(\lambda I-A)^{k-n}\left((\lambda I-A)^{-1}-(\lambda I-B)^{-1}\right)(\lambda I-B)^{1-k} .
$$

Следовательно, для любых самосопряжённых операторов $A$ и $B$ имеем:

$$
\begin{aligned}
\left\|(\lambda I-A)^{-n}-(\lambda I-B)^{-n}\right\| \\
\quad \leqslant \sum_{k=1}^{n}\left\|(\lambda I-A)^{k-n}\right\| \cdot\left\|(\lambda I-A)^{-1}-(\lambda I-B)^{-1}\right\| \cdot\left\|(\lambda I-B)^{1-k}\right\| \\
\quad \leqslant \sum_{k=1}^{n}|\operatorname{Im} \lambda|^{k-n}|\operatorname{Im} \lambda|^{-2}\|A-B\| \cdot|\operatorname{Im} \lambda|^{1-k}=n|\operatorname{Im} \lambda|^{-n-1}\|A-B\| .
\end{aligned}
$$

Таким образом, мы доказали, что $\left\|(\lambda I-x)^{-n}\right\|_{\mathrm{OL}(\mathbb{R})} \leqslant n|\operatorname{Im} \lambda|^{-n-1}$. Остаётся заметить, что $\left\|(\lambda-x)^{-n}\right\|_{\mathrm{OL}(\mathbb{R})} \geqslant\left\|(\lambda-x)^{-n}\right\|_{\operatorname{Lip}(\mathbb{R})}=n|\operatorname{Im} \lambda|^{-n-1}$. 
ПРимеР 5. Функция $x \mapsto e^{\mathrm{i} a x}, a \in \mathbb{R}$, является операторно липшицевой, и $\left\|e^{\mathrm{i} a x}\right\|_{\mathrm{OL}(\mathbb{R})}=|a|$.

ДоказАтельство. Снова достаточно установить только оценку сверху. Можно считать, что $a=1$. Пусть $A$ и $B$ - самосопряжённые операторы. Тогда

$$
\left(e^{\mathrm{i} t A} e^{-\mathrm{i} t B}\right)^{\prime}=\mathrm{i} A e^{\mathrm{i} t A} e^{-\mathrm{i} t B}-\mathrm{i} e^{\mathrm{i} t A} e^{-\mathrm{i} t B} B=\mathrm{i} e^{\mathrm{i} t A}(A-B) e^{-\mathrm{i} t B},
$$

откуда следует, что

$$
\begin{aligned}
\left\|e^{\mathrm{i} A}-e^{\mathrm{i} B}\right\| & =\left\|e^{\mathrm{i} A} e^{-\mathrm{i} B}-I\right\|=\left\|\mathrm{i} \int_{0}^{1} e^{\mathrm{i} t A}(A-B) e^{-\mathrm{i} t B} d t\right\| \\
& \leqslant \int_{0}^{1}\left\|e^{\mathrm{i} t A}(A-B) e^{-\mathrm{i} t B}\right\| d t=\int_{0}^{1}\|A-B\| d t=\|A-B\| .
\end{aligned}
$$

Во всех приведённых выше примерах имеет место равенство $\|f\|_{\mathrm{OL}(\mathbb{R})}=$ $\left\|f^{\prime}\right\|_{L^{\infty}(\mathbb{R})}$, что скорее является исключением, чем правилом.

Из примера 5 мгновенно вытекает следующее утверждение.

ТеОрема 1.1.1. Пусть $f$ - первообразная преобразования Фуръе $\mathscr{F} \mu$ комплексной борелевской меры $\mu$ на $\mathbb{R}$. Тогда $f \in \mathrm{OL}(\mathbb{R})$ u $\|f\|_{\mathrm{OL}(\mathbb{R})} \leqslant\|\mu\|$.

ДокАзАТЕЛьство. Можно считать, что $f(0)=0$. Тогда

$$
\begin{aligned}
f(x) & =\int_{0}^{x}(\mathscr{F} \mu)(t) d t=\int_{0}^{x}\left(\int_{\mathbb{R}} e^{-\mathrm{i} s t} d \mu(s)\right) d t \\
& =\int_{0}^{1}\left(\int_{\mathbb{R}} x e^{-\mathrm{i} s t x} d \mu(s)\right) d t=\mathrm{i} \int_{\mathbb{R}} \frac{e^{-\mathrm{i} s x}-1}{s} d \mu(s) .
\end{aligned}
$$
Следовательно, $\|f\|_{\mathrm{OL}(\mathbb{R})} \leqslant \int_{\mathbb{R}}\left\|\frac{e^{-\mathrm{i} s x}-1}{s}\right\|_{\mathrm{OL}(\mathbb{R})} d|\mu|(s) \leqslant \int_{\mathbb{R}} d|\mu|(s)=\|\mu\|$. Тео-
рема доказана.

СлеДСтвиЕ 1.1.2. Пусть $f \in C^{1}(\mathbb{R})$. Предположим, что функиия $f^{\prime}$ является положительно определённой. Тогда $\|f\|_{\mathrm{OL}(\mathbb{R})}=\|f\|_{\operatorname{Lip}(\mathbb{R})}=f^{\prime}(0)$.

ДокАЗАтельство. В силу классической теоремы Бохнера (см., например, [77]) положительно определённую функцию $f^{\prime}$ можно представить в виде $f^{\prime}=\mathscr{F} \mu$, где $\mu$ - конечная борелевская положительная мера н $\mathbb{R}$. Остаётся заметить, что $\|\mu\|=f^{\prime}(0)=\left|f^{\prime}(0)\right| \leqslant\|f\|_{\text {Lip }(\mathbb{R})} \leqslant\|f\|_{\mathrm{OL}(\mathbb{R})} \leqslant\|\mu\|$, где последнее неравенство вытекает из теоремы 1.1.1. Следствие доказано.

По существу все приведённые выше в этом разделе примеры явного вычисления полунормы в пространстве $\mathrm{OL}(\mathbb{R})$ так или иначе основаны на следствии 1.1.2. Тем не менее можно построить пример функции $f \in \mathrm{OL}(\mathbb{R})$ такой, что $\|f\|_{\mathrm{OL}(\mathbb{R})}=\|f\|_{\operatorname{Lip}(\mathbb{R})}=f^{\prime}(0)$ и $f$ не удовлетворяет условиям следствия 1.1.2.

$\mathrm{C}$ другой стороны, если $\|f\|_{\mathrm{OL}(\mathbb{R})}=\|f\|_{\mathrm{Lip}(\mathbb{R})}=(f(a)-f(0)) / a=1$ при $a \in \mathbb{R}$, $a \neq 0$, то $f(x)=x+f(0)$ при всех $x \in \mathbb{R}$.

Пример 5 допускает ещё одно обобщение, так называемое операторное неравенство Бернштейна. Об этом речь пойдёт в разделе 1.4. В частности, в разделе 1.4 будет показано, что $L^{\infty}(\mathbb{R}) \cap \mathscr{E}_{\sigma} \subset \mathrm{OL}(\mathbb{R})$, где символом $\mathscr{E}_{\sigma}$ обозначается пространство целых функций экспоненциального типа не выше $\sigma$.

Рассмотрим примеры операторно липшицевых функций на единичной окружности $\mathbb{T}$. 
ПримеР 6. Пусть $n \in \mathbb{Z}$. Тогда $\left\|z^{n}\right\|_{\mathrm{OL}(\mathbb{T})}=|n|$ для всех $n \in \mathbb{Z}$.

ДоказАтельство. Достаточно рассмотреть случай $n>0$, а тогда всё сводится к проверке неравенства $\left\|U^{n}-V^{n}\right\| \leqslant n\|U-V\|$ для любых унитарных операторов $U$ и $V$. Чтобы его доказать, достаточно подставить $X=U$ и $Y=V$ в тождество (1.1.1).

Из этого примера мгновенно вытекает аналог теоремы 1.1.1 для окружности.

ТеОРема 1.1.3. Пусть $f$ - непрерьвная функиия на единичной окружности $\mathbb{T}$ такая, ито $\sum_{n \in \mathbb{Z}}|n| \cdot|\widehat{f}(n)|<\infty$. Тогда

$$
f \in \mathrm{OL}(\mathbb{T}), \quad\|f\|_{\mathrm{OL}(\mathbb{T})} \leqslant \sum_{n \in \mathbb{Z}}|n| \cdot|\widehat{f}(n)| .
$$

Отметим, что более сильные результаты будут приведены в разделе 1.6.

ПримеР 7. Функция $x \mapsto x^{2} \sin (1 / x)$ является операторно липшицевой. Чтобы убедиться в этом, докажем следующую теорему.

Tеорема 1.1.4. Пусть $f \in \mathrm{OL}(\mathbb{R})$ u $f(0)=0$. Положим $g(x)=x^{2} f\left(x^{-1}\right)$, если $x \neq 0, u g(0)=0$. Тогда $g \in \mathrm{OL}(\mathbb{R}) u$

$$
\frac{1}{3}\|f\|_{\mathrm{OL}(\mathbb{R})} \leqslant\|g\|_{\mathrm{OL}(\mathbb{R})} \leqslant 3\|f\|_{\mathrm{OL}(\mathbb{R})} .
$$

ДокАзАтельство. Достаточно доказать второе неравенство, поскольку первое сводится ко второму. Можно считать, что $\|f\|_{\mathrm{OL}(\mathbb{R})}=1$. Как мы отмечали во введении к этой главе, для функций на прямой операторная липшицевость эквивалентна коммутаторной липшицевости и соответствующие нормы совпадают: $\|f\|_{\mathrm{OL}(\mathbb{R})}=\|f\|_{\mathrm{CL}(\mathbb{R})}$ (см. разделы $\left.1.3,3.1\right)$. Поэтому достаточно доказать, что неравенство

$$
\|f(A) R-R f(A)\| \leqslant\|A R-R A\|
$$

для любого ограниченного оператора $R$ и любого ограниченного самосопряжённого оператора $A$ влечёт неравенство $\|g(A) R-R g(A)\| \leqslant 3\|A R-R A\|$ для любого оператора $R$ и любого самосопряжённого оператора $A$. Предположим, что оператор $A$ обратим. В этом случае достаточно доказать неравенство

$$
\left\|A^{2} f\left(A^{-1}\right) R-R A^{2} f\left(A^{-1}\right)\right\| \leqslant 3\|A R-R A\|
$$

для любого ограниченного оператора $R$ и любого обратимого самосопряжённого оператора $A$. Имеем

$$
\begin{aligned}
f\left(A^{-1}\right) A^{2} R-R A^{2} f\left(A^{-1}\right)= & f\left(A^{-1}\right) A(A R-R A)+f\left(A^{-1}\right) A R A \\
& -A R A f\left(A^{-1}\right)+(A R-R A) A f\left(A^{-1}\right) .
\end{aligned}
$$

Ясно, что $\left\|A f\left(A^{-1}\right)\right\| \leqslant \sup _{t \neq 0}\left|t^{-1} f(t)\right| \leqslant\|f\|_{\operatorname{Lip}(\mathbb{R})} \leqslant\|f\|_{\mathrm{OL}(\mathbb{R})}=1$. Поэтому

$$
\left\|f\left(A^{-1}\right) A(A R-R A)\right\| \leqslant\|A R-R A\|, \quad\left\|(A R-R A) A f\left(A^{-1}\right)\right\| \leqslant\|A R-R A\| .
$$

Подставляя в (1.1.3) операторы $A R A$ и $A^{-1}$, получаем

$$
\left\|f\left(A^{-1}\right) A R A-A R A f\left(A^{-1}\right)\right\| \leqslant\left\|A^{-1} A R A-A R A A^{-1}\right\|=\|A R-R A\|,
$$


откуда мгновенно следует (1.1.4). Дабы рассмотреть общий случай, достаточно заметить, что для любого положительного числа $\delta$ найдётся обратимый самосопряжённый оператор $A_{\delta}$ такой, что $A A_{\delta}=A_{\delta} A$ и $\left\|A-A_{\delta}\right\|<\delta$. Тогда при всех $\delta>0$

$$
\begin{aligned}
\|g(A) R-R g(A)\| \leqslant & \left\|g(A)-g\left(A_{\delta}\right)\right\| \cdot\|R\|+\left\|g\left(A_{\delta}\right) R-R g\left(A_{\delta}\right)\right\| \\
& +\left\|g\left(A_{\delta}\right)-g(A)\right\| \cdot\|R\| \\
\leqslant & 2 \delta\|R\| \cdot\|g\|_{\operatorname{Lip}(\mathbb{R})}+3\left\|A_{\delta} R-R A_{\delta}\right\| \\
\leqslant & 6 \delta\|R\| \cdot\|f\|_{\operatorname{Lip}(\mathbb{R})}+3\|A R-R A\|+6 \delta\|R\| \\
\leqslant & 3\|A R-R A\|+12 \delta\|R\| .
\end{aligned}
$$

Теорема доказана.

ЗАмечАниЕ. Теперь ясно, ввиду примера 5, что функция $g$, определённая равенством $g(x)=x^{2} \sin (1 / x)$, операторно липшицева. Функиия $g$ даёт нам пример операторно липшищевой функции, не являющейся непрерывно дифберенцируемой. Вопрос о существовании таких функций был поставлен в [76] и решён в [36]. То, что функция $g$ операторно липшицева на любом конечном интервале, было показано в [38]. Напомним (см. теорему 3.3 .3 ниже), что всякая операторно липшицева функция на $\mathbb{R}$ должна быть дифференцируемой в каждой точке.

Отметим ещё, что в [3] доказано, что множество на прямой является множеством точек разрыва производной операторно липшицевой функции в том и только том случае, когда оно является множеством первой категории типа $F_{\sigma}$. Другими словами, множества точек разрыва производных операторно липшицевых функций устроены так же, как множества точек разрыва функций первого класса Бэра.

В разделе 3.10 теорема 1.1.4 обобщается на случай произвольных дробно-линейных преобразований.

\section{2. Операторная липшицевость в сравнении с операторной дифференцируемостью}

Пусть $H$ - функция на подмножестве $\Lambda$ вещественной прямой $\mathbb{R}$ со значениями в банаховом пространстве $X$. Функция $H$ называется липшицевой, если существует неотрицательное число $c$ такое, что

$$
\|H(s)-H(t)\|_{X} \leqslant c|s-t|, \quad s, t \in \Lambda .
$$

Множество всех таких функций обозначим через $\operatorname{Lip}(\Lambda, X)$. Пусть $\|H\|_{\operatorname{Lip}(\Lambda, X)}$ обозначает наименьшую константу $c$, удовлетворяющую условию (1.2.1). Как обычно, мы полагаем $\|H\|_{\operatorname{Lip}(\Lambda, X)} \stackrel{\text { def }}{=} \infty$, если $H \notin \operatorname{Lip}(\Lambda, X)$.

Пусть $f$ - непрерывная функция на вещественной прямой $\mathbb{R}$. C каждым самосопряжённым оператором $A$ и каждым ограниченным самосопряжённым оператором $K$ мы связываем функцию $H_{f, A, K}, H_{f, A, K}(t)=f(A+t K)-f(A)$, заданную при всех $t$ из $\mathbb{R}$ таких, что $f(A+t K)-f(A) \in \mathcal{B}(\mathscr{H})$.

Заметим, что если $f \in \mathrm{OL}(\mathbb{R})$, то

$$
H_{f, A, K} \in \operatorname{Lip}(\mathbb{R}, \mathcal{B}(\mathscr{H})) \quad \text { и } \quad\left\|H_{f, A, K}\right\|_{\operatorname{Lip}(\mathbb{R}, \mathcal{B}(\mathscr{H}))} \leqslant\|K\| \cdot\|f\|_{\mathrm{OL}(\mathbb{R})} .
$$


Легко видеть, что имеет место следующее утверждение.

Лемма 1.2.1. Пусть $f$-непрерывная функиия на $\mathbb{R}$. Тогда

$$
\begin{aligned}
\|f\|_{\mathrm{OL}(\mathbb{R})} & =\sup \left\{\left\|H_{f, A, K}\right\|_{\operatorname{Lip}(\mathbb{R}, \mathcal{B}(\mathscr{H}))}: A, K \in \mathcal{B}_{\mathrm{sa}}(\mathscr{H}),\|K\|=1\right\} \\
& =\sup \left\{\left\|H_{f, A, K}\right\|_{\operatorname{Lip}(\mathbb{R}, \mathcal{B}(\mathscr{H}))}: K \in \mathcal{B}_{\mathrm{sa}}(\mathscr{H}),\|K\|=1, A^{*}=A\right\} .
\end{aligned}
$$

Нам понадобится следующее хорошо известное элементарное утверждение. Для удобства читателя мы приведём здесь одно из возможных доказательств.

ЛЕмма 1.2.2. Пусть $H$ - функиия со значениями в банаховом пространстве $X$, заданная на невырожденном промежутке $\Lambda, \Lambda \subset \mathbb{R}$. Тогда

$$
\|H\|_{\operatorname{Lip}(\Lambda, X)}=\sup _{t \in \Lambda} \varlimsup_{h \rightarrow 0} \frac{\|H(t+h)-H(t)\|_{X}}{|h|} .
$$

ДокАзАтельство. Неравенство “”” очевидно. Чтобы доказать противоположное неравенство, достаточно убедиться в том, что неравенство (1.2.1) справедливо, как только $c$ удовлетворяет условию

$$
c>\sup _{t \in \Lambda} \varlimsup_{h \rightarrow 0} \frac{\|H(t+h)-H(t)\|_{X}}{|h|} .
$$

Зафиксируем такое число $c$ и произвольную точку $t$ из $\Lambda$. Пусть $\Lambda_{t}-$ множество всех точек $s$ из $\Lambda$, удовлетворяющих неравенству (1.2.1). Из (1.2.2) мгновенно вытекает, что множество $\Lambda_{t}$ является одновременно открытым и замкнутым в $\Lambda$. Кроме того, $\Lambda_{t} \neq \varnothing$, ибо $t \in \Lambda$. Следовательно, $\Lambda_{t}=\Lambda$ в силу связности промежутка $\Lambda$. Лемма доказана.

ТеОрема 1.2.3. Пусть $f$ - непрерывная функция на вещественной прямой $\mathbb{R}$. Предположим, что

$$
\varlimsup_{t \rightarrow 0} \frac{\|f(A+t K)-f(A)\|}{|t|}<+\infty
$$

для любого (не обязательно ограниченного) самосопряжённого оператора $A$ и любого ограниченного самосопряжённого оператора $K$. Тогда $f \in \mathrm{OL}(\mathbb{R})$.

ДокАЗАТЕЛьСтво. Из лемм 1.2.1 и 1.2.2 легко вытекает, что

$$
\|f\|_{\mathrm{OL}(\mathbb{R})}=\sup \left\{\varlimsup_{t \rightarrow 0} \frac{\|f(A+t K)-f(A)\|}{|t|}: A, K \in \mathcal{B}_{\mathrm{sa}}(\mathscr{H}),\|K\|=1\right\} .
$$

Таким образом, если предположить, что $\|f\|_{\mathrm{OL}(\mathbb{R})}=\infty$, то для каждого числа $n$ из $\mathbb{Z}_{+}$найдутся операторы $A_{n}, K_{n} \in \mathcal{B}_{\text {sa }}(\mathscr{H})$ такие, что $\left\|K_{n}\right\|=1$ и

$$
\varlimsup_{t \rightarrow 0} \frac{\left\|f\left(A_{n}+t K_{n}\right)-f\left(A_{n}\right)\right\|}{|t|}>n .
$$

Рассмотрим в гильбертовом пространстве $\ell^{2}(\mathscr{H})$ самосопряжённые операторы $\mathscr{A}, \mathcal{K}$, определённые равенствами

$$
\begin{aligned}
\mathscr{A}\left(x_{0}, x_{1}, x_{2}, \ldots\right)=\left(A_{0} x_{0}, A_{1} x_{1}, A_{2} x_{2}, \ldots\right), & \left(x_{0}, x_{1}, x_{2}, \ldots\right) \in \ell^{2}(\mathscr{H}), \\
\mathcal{K}\left(x_{0}, x_{1}, x_{2}, \ldots\right)=\left(K_{0} x_{0}, K_{1} x_{1}, K_{2} x_{2}, \ldots\right), & \left(x_{0}, x_{1}, x_{2}, \ldots\right) \in \ell^{2}(\mathscr{H}) .
\end{aligned}
$$


Тогда

$$
\varlimsup_{t \rightarrow 0} \frac{\|f(\mathscr{A}+t \mathcal{K})-f(\mathscr{A})\|}{|t|} \geqslant \varlimsup_{t \rightarrow 0} \frac{\left\|f\left(A_{n}+t K_{n}\right)-f\left(A_{n}\right)\right\|}{|t|}>n
$$

для любого целого неотрицательного числа $n$, и мы приходим к противоречию. Теорема доказана.

ЗАмечАниЕ. Из доказательства теоремы 1.2.3 можно увидеть, что имеют место следующие равенства:

$$
\begin{aligned}
\|f\|_{\mathrm{OL}(\mathbb{R})} & =\sup \left\{\varlimsup_{t \rightarrow 0} \frac{\|f(A+t K)-f(A)\|}{|t|}: A, K \in \mathcal{B}_{\mathrm{sa}}(\mathscr{H}),\|K\|_{\mathcal{B}_{\mathrm{sa}}(\mathscr{H})}=1\right\} \\
& =\sup \left\{\left\|H_{f, A, K}\right\|_{\mathrm{Lip}(\mathbb{R})}: A, K \in \mathcal{B}_{\mathrm{sa}}(\mathscr{H}),\|K\|_{\mathcal{B}_{\mathrm{sa}}(\mathscr{H})}=1\right\} .
\end{aligned}
$$

Предваряя следующую теорему, заметим, что функция $H_{f, A, K}$ дифференцируема при всех самосопряжённых операторах $A$ и $K$ тогда и только тогда, когда она дифференцируема в нуле для любых самосопряжённых операторов $A$ и $K$ (как обычно, оператор $K$ предполагается ещё и ограниченным).

Доказательство следующей теоремы использует теорему 3.5.6 главы III.

Теорема 1.2.4. Пусть $f$-непрерывная функиия на $\mathbb{R}$. Тогда включение

(a) $f \in \mathrm{OL}(\mathbb{R})$

равносильно выполнению каждого из следующих условий для любого самосопряжённого оператора $A$ и любого ограниченного самосопряжённого оператоpa $K$ :

(b) $H_{f, A, K} \in \operatorname{Lip}(\mathbb{R}, \mathcal{B}(\mathscr{H}))$;

(c) функиия $H_{f, A, K}$ дифференцируема как функция из $\mathbb{R}$ в пространство $\mathcal{B}(\mathscr{H})$, наделённое слабой операторной топологией;

(d) функиия $H_{f, A, K}$ дифферениируема как функиия из $\mathbb{R}$ в пространство $\mathcal{B}(\mathscr{H})$, наделённое силъной операторной топологией.

ДоказАтельство. Импликации (a) $\Rightarrow(\mathrm{b})$ и $(\mathrm{d}) \Rightarrow(\mathrm{c})$ очевидны. Импликация $(\mathrm{a}) \Rightarrow(\mathrm{d})$ вытекает из теоремы 3.5.6 ниже, а импликации (c) $\Rightarrow$ (a) и (b) $\Rightarrow$ (a) мгновенно следуют из теоремы 1.2.3. Теорема доказана.

Обозначим символом $\mathrm{OL}_{\mathrm{loc}}(\mathbb{R})$ пространство непрерывных на $\mathbb{R}$ функций $f$ таких, что $f \mid[-a, a] \in \mathrm{OL}([-a, a])$ для всех $a>0$, и символом $\operatorname{Lip}_{\text {loc }}(\mathbb{R}, \mathcal{B}(\mathscr{H}))$ пространство непрерывных на $\mathbb{R}$ функций $f$ таких, что $f \mid[-a, a] \in \operatorname{Lip}([-a, a]$, $\mathcal{B}(\mathscr{H}))$ для всех $a>0$. Все результаты этого раздела имеют естественные аналоги и для этих пространств.

ТеОРема 1.2.5. Пусть $f$ - непрерывная функция на вещественной прямой $\mathbb{R}$. Предположим, что

$$
\varlimsup_{t \rightarrow 0} \frac{\|f(A+t K)-f(A)\|}{|t|}<\infty
$$

для любых $A, K \in \mathcal{B}_{\mathrm{sa}}(\mathscr{H})$. Тогда $f \in \mathrm{OL}_{\mathrm{loc}}(\mathbb{R})$.

ДоказАтельство. Предположим, что $f \notin \mathrm{OL}_{\mathrm{loc}}(\mathbb{R})$. Тогда $f \notin \mathrm{OL}([-a, a])$ для некоторого числа $a>0$. Следовательно, для любого числа $c \geqslant 0$ существуют операторы $A, K$ из $\mathcal{B}_{\mathrm{sa}}(\mathscr{H})$ такие, что $\|A\| \leqslant a,\|A+K\| \leqslant a$ 
и $\|f(A+K)-f(A)\|>c\|K\|$. Повторяя рассуждения доказательства теоремы 1.2.3, мы придём к противоречию, построив самосопряжённые операторы $\mathscr{A}$ и $\mathscr{A}+\mathcal{K}$ такие, что

$$
\|\mathscr{A}\| \leqslant a, \quad\|\mathscr{A}+\mathcal{K}\| \leqslant a \quad \text { и } \quad \varlimsup_{t \rightarrow 0} \frac{\|f(\mathscr{A}+t \mathcal{K})-f(\mathscr{A})\|}{|t|}=\infty .
$$

Теорема доказана.

ТеОрема 1.2.6. Пусть $f$ - непрерывная функиия на $\mathbb{R}$. Тогда следующие утверждения равносильны:

(a) $f \in \mathrm{OL}_{\mathrm{loc}}(\mathbb{R})$;

(b) $H_{f, A, K} \in \operatorname{Lip}_{\text {loc }}(\mathbb{R}, \mathcal{B}(\mathscr{H}))$ для любъх $A, K \in \mathcal{B}_{\mathrm{sa}}(\mathscr{H})$;

(c) для любых $A, K$ из $\mathcal{B}_{\mathrm{sa}}(\mathscr{H})$ функиия $H_{f, A, K}$ дифферениируема как функиия из $\mathbb{R}$ в пространство $\mathcal{B}(\mathscr{H})$, наделённое слабой операторной топологией;

(d) для любых $A, K$ из $\mathcal{B}_{\mathrm{sa}}(\mathscr{H})$ функиия $H_{f, A, K}$ дифференцируема как функиця из $\mathbb{R}$ в пространство $\mathcal{B}(\mathscr{H})$, наделённое сильной операторной топологией.

Эта теорема может быть доказана аналогично теореме 1.2.4, только вместо теоремы 1.2.3 нужно использовать теорему 1.2.5.

Отметим, что в работе [37] показано, что условие (а) в теореме 1.2.6 эквивалентно дифференцируемости по норме по всем компактным направлениям для ограниченных самосопряжённых операторов.

Из теоремы 1.2 .4 следует, что если $f$ - непрерывная функция на $\mathbb{R}$, то $f \in \mathrm{OL}(\mathbb{R})$ в том и только том случае, когда для любого самосопряжённого оператора $A$ и любого ограниченного самосопряжённого оператора $K$ существует предел

$$
\lim _{t \rightarrow 0} \frac{1}{t}(f(A+t K)-f(A)) \stackrel{\text { def }}{=} \boldsymbol{d}_{f, A} K
$$

в сильной операторной топологии. Из теоремы 3.5.6 главы III следует также, что $\boldsymbol{d}_{f, A}-$ ограниченный линейный оператор, действующий из $\mathcal{B}_{\mathrm{sa}}(\mathscr{H})$ в $\mathcal{B}(\mathscr{H})$.

Аналогичные утверждения имеют место и для функций $f \in \mathrm{OL}_{\mathrm{loc}}(\mathbb{R})$, с той лишь разницей, что теперь оператор $A$ должен быть ещё и ограниченным.

Из теоремы 1.2.6 вытекает, что если $f$ - непрерывная функция на $\mathbb{R}$, то $f \in \mathrm{OL}_{\text {loc }}(\mathbb{R})$ в том и только том случае, когда для любых операторов $A, K$ из $\mathcal{B}_{\mathrm{sa}}(\mathscr{H})$ предел (1.2.5) существует в сильной операторной топологии, при этом $\boldsymbol{d}_{f, A}$ - ограниченный линейный оператор из $\mathcal{B}_{\text {sa }}(\mathscr{H})$ в $\mathcal{B}(\mathscr{H})$.

TeOpema 1.2.7. Пусть $f \in \mathrm{OL}_{\mathrm{loc}}(\mathbb{R})$. Тогда

$$
\|f\|_{\mathrm{OL}(\mathbb{R})}=\sup _{A \in \mathcal{B}_{\mathrm{sa}}(\mathscr{H})}\left\|\boldsymbol{d}_{f, A}\right\|=\sup \left\{\left\|\boldsymbol{d}_{f, A}\right\|: A-\text { самосопряжё̈нный оператор }\right\} .
$$

Как обычно, равенство $\|f\|_{\mathrm{OL}(\mathbb{R})}=\infty$ означает, что $f \notin \mathrm{OL}(\mathbb{R})$.

ДокАЗАТЕЛЬСТво. Достаточно воспользоваться леммой 1.2.1.

ТеОрема 1.2.8. Пусть $f$-непрерывная функиия на $\mathbb{R}$. Предположим, что для любого самосопряжённого оператора $A$ и любого ограниченного самосопряжённого оператора $K$ предел (1.2.5) существует по операторной норме. Тогда $f \in \mathrm{OL}(\mathbb{R}) \cap C^{1}(\mathbb{R})$, отображсение $K \mapsto f(A+K)-f(A)\left(K \in \mathcal{B}_{\mathrm{sa}}(\mathscr{H})\right)$ дифберениируемо по Фреше в точке 0 при каждом самосопряжённом операторе $A$ и его дифференииал в точке $\mathbf{0}$ равен $\boldsymbol{d}_{f, A}$. 
Доказательство. Включение $f \in \mathrm{OL}(\mathbb{R})$ вытекает из теоремы 1.2.4. Из теоремы 3.5.6 следует, что $\boldsymbol{d}_{f, A}$ - ограниченный линейный оператор, действующий из $\mathcal{B}_{\mathrm{sa}}(\mathscr{H})$ в $\mathcal{B}(\mathscr{H})$. Проверим дифферениируемость по Фреше, т. е. что $\boldsymbol{d}_{f, A}$ - линейный ограниченный оператор (уже доказано) и $\lim _{t \rightarrow 0} t^{-1} \| f(A+$ $t K)-f(A)-t \boldsymbol{d}_{f, A} K \|=0$ равномерно по всем $K$ из единичной сферы пространства $\mathcal{B}_{\text {sa }}(\mathscr{H})$.

Для этого достаточно убедиться в том, что

$$
\lim _{n \rightarrow \infty} \frac{1}{t_{n}}\left\|f\left(A+t_{n} K_{n}\right)-f(A)-t_{n} \boldsymbol{d}_{f, A} K_{n}\right\|=0
$$

для любой последовательности ненулевых вещественных чисел $\left\{t_{n}\right\}_{n \geqslant 0}$, стремящейся к нулю, и для любой последовательности самосопряжённых операторов $\left\{K_{n}\right\}_{n \geqslant 0}$ такой, что $\left\|K_{n}\right\|=1$ при всех $n$.

Рассмотрим в гильбертовом пространстве $\ell^{2}(\mathscr{H})$ самосопряжённый оператор $\mathscr{A}$ и ограниченный самосопряжённый оператор $\mathcal{K}$, определённые равенствами (1.2.3) при $A_{n}=A$ и (1.2.4). Применяя условие теоремы к операторам $\mathscr{A}$ и $\mathcal{K}$, получаем:

$$
\lim _{n \rightarrow \infty} \frac{1}{t_{n}}\left\|f\left(\mathscr{A}+t_{n} \mathcal{K}\right)-f(\mathscr{A})-t_{n} \boldsymbol{d}_{f, \mathscr{A}} \mathcal{K}\right\|=0 .
$$

Ясно, что $\boldsymbol{d}_{f, \mathscr{A}} \mathcal{K}$ - это ортогональная сумма операторов $\boldsymbol{d}_{f, A} K_{n}, n \geqslant 0$, и, таким образом, (1.2.6) является следствием равенства (1.2.7).

Докажем, наконец, что $f \in C^{1}(\mathbb{R})$. Проверим непрерывность функции $f^{\prime}$ в произвольной точке $t_{0}$. Пусть $A$ - оператор умножения на $x$ в $L^{2}\left(\left[x_{0}-1\right.\right.$, $\left.\left.x_{0}+1\right]\right)$. Положим $K \stackrel{\text { def }}{=} I$. Тогда по условию теоремы существует предел $\lim _{t \rightarrow 0} t^{-1}(f(A+t I)-f(A))$ по операторной норме. Следовательно, существует предел $\lim _{t \rightarrow 0} t^{-1}(f(x+t)-f(x))=f^{\prime}(x)$ в $L^{\infty}\left(\left[x_{0}-1, x_{0}+1\right]\right)$, откуда $f \in$ $C^{1}\left(t_{0}-1, t_{0}+1\right)$. Теорема доказана.

ОПРЕДЕЛЕНИЕ. Функция $f$, удовлетворяющая условиям теоремы 1.2 .8 , называется операторно дифберениируемой. Обозначим символом $\mathrm{OD}(\mathbb{R})$ множество всех операторно дифференцируемых функций на $\mathbb{R}$.

Напомним, что для функций, определённых на банаховых пространствах, есть разные понятия дифференцируемости: существование слабой производной по Гато, существование дифференциала Гато, дифференцируемость по Фреше. Однако, как видно из теоремы 1.2.8, в случае операторной дифференцируемости функций на прямой все эти определения эквивалентны. Отметим, что эквивалентность операторной дифференцируемости по Фреше и существования дифференциала Гато, являющегося линейным ограниченным оператором, доказана в работе [37].

Следующее утверждение можно доказать примерно так же, как теорему 1.2.8.

Теорема 1.2.9. Пусть $f$-непрерывная функиия на $\mathbb{R}$. Предположим, что для любых $A, K \in \mathcal{B}_{\mathrm{sa}}$ предел (1.2.5) существует по операторной норме. Тогда $f \in \mathrm{OL}_{\mathrm{loc}}(\mathbb{R}) \cap C^{1}(\mathbb{R})$, отображение $K \mapsto f(A+K)-f(A), K \in \mathcal{B}_{\mathrm{sa}}$, дифберениируемо по Фреше в точке $\mathbf{0}$ при всех $A \in \mathcal{B}_{\text {sa }}$ и его дифференииал в точке $\mathbf{0}$ равен $\boldsymbol{d}_{f, A}$. 
Если функция $f$ удовлетворяет условиям теоремы 1.2 .9 , то говорят, что она локально операторно дифферениируема, и пишут $f \in \mathrm{OD}_{\mathrm{loc}}(\mathbb{R})$.

Обратим внимание на то, что теоремы 1.2.8 и 1.2.9 утверждают, в частности, что если $f \in \mathrm{OD}_{\mathrm{loc}}(\mathbb{R})$, то функиия $f$ непрерьвно дифферениируема и входит в класс $\mathrm{OL}_{\mathrm{loc}}(\mathbb{R})$, а если $f \in \mathrm{OD}(\mathbb{R})$, mо $f \in \mathrm{OL}(\mathbb{R})$.

ЗАмечАниЕ. Отметим, что, не будучи непрерывно дифференцируемой, функция $g, g(x)=x^{2} \sin (1 / x)$, не может быть операторно дифференцируемой. Таким образом, в теореме 1.1.4 нельзя заменить класс операторно липшицевых функций классом операторно дифференцируемых функций. Действительно, нетрудно проверить, что функция $x \mapsto \sin x=\operatorname{Im} e^{\mathrm{i} x}$ является операторно дифференцируемой.

Нашей ближайшей целью является доказательство непрерывной зависимости (по операторной норме) дифференциала $\boldsymbol{d}_{f, A}$ от оператора $A$ для (локально) операторно дифференцируемых функций $f$. Следующий результат был получен в работе [37].

Теорема 1.2.10. Пусть $f$ - локалъно операторно дифферениируемая функиия, и пусть с > 0. Тогда для любого положительного числа в существует положительное число $\delta$ такое, что $\left\|\boldsymbol{d}_{f, A}-\boldsymbol{d}_{f, B}\right\| \leqslant \varepsilon$, как только $A$ u $B-$ самосопряжённые операторь такие, что $\|A\| \leqslant c,\|B\| \leqslant c u\|A-B\| \leqslant \delta$.

Докажем сначала следующую лемму, полученную в работе [37].

Лемма 1.2.11. Пусть $f$ - локально операторно дифференцируемая функиця. Тогда для любых положительных чисел с и $\varepsilon$ найдётся число $\delta>0$ maкое, что

$$
\left\|f(A+K)-f(A)-\boldsymbol{d}_{f, A} K\right\| \leqslant \varepsilon\|K\|,
$$

как только $A$ и $K$ - самосопряжённъе операторь такие, что $\|K\| \leqslant \delta u$ $\|A\| \leqslant c$.

ДокАЗАТЕЛЬство. Предположим противное. Тогда для некоторых положительных чисел $c$ и $\varepsilon$ найдутся последовательности самосопряжённых операторов $\left\{A_{n}\right\}_{n \geqslant 0}$ и $\left\{K_{n}\right\}_{n \geqslant 0}$ такие, что $\left\|K_{n}\right\| \rightarrow 0,\left\|A_{n}\right\| \leqslant c$ и

$$
\left\|f\left(A_{n}+K_{n}\right)-f\left(A_{n}\right)-\boldsymbol{d}_{f, A_{n}} K_{n}\right\|>\varepsilon\left\|K_{n}\right\|, \quad n \geqslant 0 .
$$

Пусть $\mathscr{A}$ - ограниченный самосопряжённый оператор в $\ell^{2}(\mathscr{H})$, определённый равенством (1.2.3). Тогда $\|A\| \leqslant c$. Поскольку функция $f$ дифференцируема по Фреше в точке $\mathscr{A}$, существует число $\delta>0$ такое, что

$$
\left\|f(\mathscr{A}+K)-f(\mathscr{A})-\boldsymbol{d}_{f, \mathscr{A}} K\right\| \leqslant \varepsilon\|K\|
$$

для любого самосопряжённого оператора $K$ с нормой $\|K\| \leqslant \delta$. Определим теперь оператор $\mathcal{K}_{n}$ в $\ell^{2}(\mathscr{H})$ равенством

$$
\mathcal{K}_{n}\left(x_{0}, x_{1}, x_{2}, \ldots\right)=\left(\mathbf{0}, \ldots, \mathbf{0}, K_{n} x_{n}, \mathbf{0}, \mathbf{0}, \ldots\right), \quad\left(x_{0}, x_{1}, x_{2}, \ldots\right) \in \ell^{2}(\mathscr{H}) .
$$

Применяя неравенство (1.2.9) при достаточно больших $n$, получаем

$$
\begin{aligned}
\left\|f\left(A_{n}+K_{n}\right)-f\left(A_{n}\right)-\boldsymbol{d}_{f, A_{n}} K_{n}\right\| & =\left\|f\left(\mathscr{A}+\mathcal{K}_{n}\right)-f(\mathscr{A})-\boldsymbol{d}_{f, \mathscr{A}} \mathcal{K}_{n}\right\| \\
& \leqslant \varepsilon\left\|\mathcal{K}_{n}\right\|=\varepsilon\left\|K_{n}\right\|,
\end{aligned}
$$

что противоречит неравенству (1.2.8). Лемма доказана. 
ДокаЗАТЕЛЬСТво теоремы 1.2.10. Пусть $c$, $\varepsilon$ и $\delta$ обозначают то же, что в лемме 1.2.11. Рассмотрим самосопряжённые операторы $A$ и $B$ такие, что $\|A\| \leqslant c,\|B\| \leqslant c / 2$ и $\|B-A\| \leqslant \min \{\delta / 2, c / 2\}$. Пусть $K-$ самосопряжённый оператор такой, что $\|K\|=\delta / 2$. Тогда $\|B+K\| \leqslant c,\|B-A\| \leqslant\|K\|$ и $\|B-A+K\| \leqslant 2\|K\|$. Следовательно,

$$
\begin{aligned}
\left\|f(B+K)-f(B)-\boldsymbol{d}_{f, B} K\right\| & \leqslant \varepsilon\|K\|, \\
\left\|f(B)-f(A)-\boldsymbol{d}_{f, A}(B-A)\right\| & \leqslant \varepsilon\|B-A\| \leqslant \varepsilon\|K\|, \\
\left\|f(B+K)-f(A)-\boldsymbol{d}_{f, A}(B-A+K)\right\| & \leqslant \varepsilon\|B-A+K\| \leqslant 2 \varepsilon\|K\| .
\end{aligned}
$$

Используя равенство $\boldsymbol{d}_{f, A}(B-A+K)=\boldsymbol{d}_{f, A}(B-A)+\boldsymbol{d}_{f, A} K$, получаем

$$
\begin{aligned}
\left\|\boldsymbol{d}_{f, B} K-\boldsymbol{d}_{f, A} K\right\| \leqslant \| & \boldsymbol{d}_{f, B} K-f(B+K)+f(B) \| \\
& +\left\|f(B+K)-f(A)-\boldsymbol{d}_{f, A}(B-A+K)\right\| \\
& +\left\|\boldsymbol{d}_{f, A}(B-A)-f(B)+f(A)\right\| \leqslant 4 \varepsilon\|K\|,
\end{aligned}
$$

откуда вытекает, что $\left\|\boldsymbol{d}_{f, B}-\boldsymbol{d}_{f, A}\right\| \leqslant 4 \varepsilon$. Теорема доказана.

Перейдём к случаю операторно дифференцируемых функций. Будем говорить, что не обязательно ограниченные самосопряжённые операторы $A$ и $B$ эквивалентны, если существует оператор $K$ из $\mathcal{B}_{\text {sa }}(\mathscr{H})$ такой, что $B=A+K$. Для операторов из одного класса можно ввести метрику $\operatorname{dist}(A, B) \stackrel{\text { def }}{=}\|B-A\|$.

Теорема 1.2.12. Пусть $f$ - операторно дифберениируемая функиия на $\mathbb{R}$. Тогда отображение $A \mapsto \boldsymbol{d}_{f, A}$ на каждом классе эквивалентности непрерывно в операторной норме.

ЛЕмма 1.2.13. Пусть $f$ - операторно дифберениируемая функиия на $\mathbb{R}$. Тогда для любого положительного числа є найдётся положительное число $\delta$ такое, что

$$
\left\|f(A+K)-f(A)-\boldsymbol{d}_{f, A} K\right\| \leqslant \varepsilon\|K\|
$$

для любого (не обязательно ограниченного) самосопряжённого оператора $A$ и для любого самосопряжённого оператора $K$, норма которого не больше, чем $\delta$.

ДокАЗАТЕЛЬСтво. Предположим противное. Тогда для некоторого числа $\varepsilon>0$ найдутся две последовательности самосопряжённых операторов $\left\{A_{n}\right\}_{n=1}^{\infty}$ и $\left\{K_{n}\right\}_{n=1}^{\infty}$ такие, что $\left\|K_{n}\right\| \rightarrow 0$ и

$$
\left\|f\left(A_{n}+K_{n}\right)-f\left(A_{n}\right)-\boldsymbol{d}_{f, A_{n}} K_{n}\right\|>\varepsilon\left\|K_{n}\right\|
$$

при всех $n \geqslant 1$. Пусть $\mathscr{A}$ и $\mathcal{K}_{n}$ - операторы в пространстве $\ell^{2}(\mathscr{H})$, определённые формулами (1.2.3) и (1.2.10). Поскольку функция $f$ дифференцируема по $\Phi$ реше в точке $\mathscr{A}$, существует положительное число $\delta$ такое, что

$$
\left\|f(\mathscr{A}+K)-f(\mathscr{A})-\boldsymbol{d}_{f, \mathscr{A}} K\right\| \leqslant \varepsilon\|K\|
$$

для всех самосопряжённых операторов $K$ нормы не выше $\delta$. Применяя это неравенство к оператору $\mathcal{K}_{n}$ при достаточно больших $n$, получаем:

$$
\begin{aligned}
& \left\|f\left(A_{n}+K_{n}\right)-f\left(A_{n}\right)-\boldsymbol{d}_{f, A_{n}} K_{n}\right\| \\
& \quad=\left\|f\left(\mathscr{A}+\mathcal{K}_{n}\right)-f(\mathscr{A})-\boldsymbol{d}_{f, \mathscr{A}} \mathcal{K}_{n}\right\| \leqslant \varepsilon\left\|\mathcal{K}_{n}\right\|=\varepsilon\left\|K_{n}\right\|,
\end{aligned}
$$

что противоречит неравенству (1.2.11). Лемма доказана. 
ДоКАЗАТЕЛЬСТВо теоремы 1.2.12. Пусть $\varepsilon$ и $\delta$ обозначают то же, что в лемме 1.2.13. Рассмотрим самосопряжённые операторы $A$ и $B$ такие, что $\|B-A\| \leqslant \delta / 2$. Пусть $K-$ самосопряжённый оператор такой, что $\|K\|=\delta / 2$. Тогда $\|B-A\| \leqslant\|K\|$ и $\|B-A+K\| \leqslant 2\|K\|$. Следовательно,

$$
\begin{aligned}
\left\|f(B+K)-f(B)-\boldsymbol{d}_{f, B} K\right\| & \leqslant \varepsilon\|K\|, \\
\left\|f(B)-f(A)-\boldsymbol{d}_{f, A}(B-A)\right\| & \leqslant \varepsilon\|B-A\| \leqslant \varepsilon\|K\|, \\
\left\|f(B+K)-f(A)-\boldsymbol{d}_{f, A}(B-A+K)\right\| & \leqslant \varepsilon\|B-A+K\| \leqslant 2 \varepsilon\|K\| .
\end{aligned}
$$

Используя равенство $\boldsymbol{d}_{f, A}(B-A+K)=\boldsymbol{d}_{f, A}(B-A)+\boldsymbol{d}_{f, A} K$, получаем:

$$
\begin{aligned}
\left\|\boldsymbol{d}_{f, B} K-\boldsymbol{d}_{f, A} K\right\| \leqslant & \left\|\boldsymbol{d}_{f, B} K-f(B+K)+f(B)\right\| \\
& +\left\|f(B+K)-f(A)-\boldsymbol{d}_{f, A}(B-A+K)\right\| \\
& +\left\|\boldsymbol{d}_{f, A}(B-A)-f(B)+f(A)\right\| \leqslant 4 \varepsilon\|K\|
\end{aligned}
$$

для всех самосопряжённых операторов $K$ таких, что $\|K\|=\delta / 2$, откуда вытекает, что $\left\|\boldsymbol{d}_{f, B}-\boldsymbol{d}_{f, A}\right\| \leqslant 4 \varepsilon$ всякий раз, когда $\|B-A\| \leqslant \delta / 2$. Теорема доказана.

Tеорема 1.2.14. Пусть $f \in \mathrm{OL}_{\mathrm{loc}}(\mathbb{R})$. Тогда функиия $f$ локально операторно дифберениируема в том и только том случае, если отображение $A \mapsto \boldsymbol{d}_{f, A}$ действует непрерьвно как отображение из банахова пространства $\mathcal{B}_{\mathrm{sa}}(\mathscr{H})$ в банахово пространство ограниченных операторов из $\mathcal{B}_{\mathrm{sa}}(\mathscr{H})$ в $\mathcal{B}(\mathscr{H})$.

ДокАЗАТЕЛЬСтво. Из теоремы 1.2 .10 следует, что достаточно проверить только, что непрерывность отображения $A \mapsto \boldsymbol{d}_{f, A}$ влечёт операторную дифференцируемость. Заметим, что $H_{f, A, K}^{\prime}(s)=\boldsymbol{d}_{f, A+s K} K$ (производная берётся в сильной операторной топологии). Следовательно,

$$
f(A+K)-f(A)=\int_{0}^{1}\left(\boldsymbol{d}_{f, A+s K} K\right) d s,
$$

где интеграл понимается в следующем смысле:

$$
(f(A+K)-f(A)) u=\int_{0}^{1}\left(\left(\boldsymbol{d}_{f, A+s K} K\right) u\right) d s
$$

для любого $u \in \mathscr{H}$. Применяя (1.2.12) к оператору $t K$ вместо $K$, получаем:

$$
\frac{1}{t}(f(A+K)-f(A))-\boldsymbol{d}_{f, A} K=\int_{0}^{1}\left(\left(\boldsymbol{d}_{f, A+s t K}-\boldsymbol{d}_{f, A}\right) K\right) d s .
$$

Будем считать, что $\|K\|=1$. Тогда из последнего тождества следует, что

$$
\left\|\frac{1}{t}(f(A+K)-f(A))-\boldsymbol{d}_{f, A} K\right\| \leqslant \int_{0}^{1}\left\|\boldsymbol{d}_{f, A+s t K}-\boldsymbol{d}_{f, A}\right\| d s .
$$

Остаётся заметить, что $\lim _{t \rightarrow 0} \int_{0}^{1}\left\|\boldsymbol{d}_{f, A+s t K}-\boldsymbol{d}_{f, A}\right\| d s=0$ равномерно по всем самосопряжённым операторам $K$ единичной нормы в силу непрерывности отображения $A \mapsto \boldsymbol{d}_{f, A}$. Теорема доказана. 
Аналогичным образом можно доказать следующее утверждение.

Теорема 1.2.15. Пусть $f \in \mathrm{OL}_{\mathrm{loc}}(\mathbb{R})$. Тогда функиия $f$ операторно дифференцируема в том и только том случае, если отображение $A \mapsto \boldsymbol{d}_{f, A}$ непреръвно в операторной норме на каждом классе эквивалентности.

ТЕОРема 1.2.16. Множество $\mathrm{OD}(\mathbb{R})$ является замкнутым подпространством пространства $\mathrm{OL}(\mathbb{R})$.

ДокАЗАТЕЛЬСтво. Нам нужно доказать, что если $\lim _{n \rightarrow \infty} f_{n}=f$ в пространстве $\mathrm{OL}(\mathbb{R})$ и $f_{n} \in \mathrm{OD}(\mathbb{R})$ при всех $n$, то $f \in \mathrm{OD}(\mathbb{R})$. Из теорем 3.5.6 и 3.3.6 следует, что

$$
\left\|\boldsymbol{d}_{f_{n}, A}-\boldsymbol{d}_{f, A}\right\|=\left\|\boldsymbol{d}_{f_{n}-f, A}\right\| \leqslant\left\|\mathfrak{D}\left(f_{n}-f\right)\right\|_{\mathfrak{M}(\mathbb{R} \times \mathbb{R})}=\left\|f_{n}-f\right\|_{\mathrm{OL}(\mathbb{R})} \rightarrow 0
$$

при $n \rightarrow \infty$. Таким образом, $\lim _{n \rightarrow \infty} \boldsymbol{d}_{f_{n}, A}=\boldsymbol{d}_{f, A}$ по норме равномерно по всем самосопряжённым операторам $A$. Теперь можно сослаться на теорему 1.2.15, поскольку непрерывность сохраняется при равномерной сходимости. Теорема доказана.

Отметим здесь, что в случае функций на конечных интервалах замкнутость множества операторно дифференцируемых функций в пространстве операторно липшицевых функций была установлена в [37]; причём там же было показано, что в этом же случае пространство операторно дифференцируемых функций совпадает с замыканием множества полиномов в пространстве операторно липшицевых функций. Отметим также, что вопрос об операторной дифференцируемости дифференцируемых функций был поставлен Видомом в [75].

\section{3. Коммутаторная липшицевость}

Напомним, что непрерывная функция $f$ на $\mathbb{R}$ называется коммутаторно липшицевой, если

$$
\|f(A) R-R f(A)\| \leqslant \text { const }\|A R-R A\|
$$

для любого ограниченного самосопряжённого оператора $A$ и для любого ограниченного оператора $R$. Как и в определении операторно липшицевых функций, если функция $f$ коммутаторно липшицева, то неравенство (1.3.1) справедливо для любого (не обязательно ограниченного) самосопряжённого оператора $A$ и для любого ограниченного оператора $R$ (см. теорему 3.2.1).

Позже мы увидим, что справедливо следующее утверждение.

Теорема 1.3.1. Пусть $f$ - непрерьвная функиия на $\mathbb{R}$. Тогда следующие условия эквивалентны:

(a) $\|f(A)-f(B)\| \leqslant\|A-B\|$ для любых самосопряжённых операторов $A, B$;

(b) $\|f(A) R-R f(A)\| \leqslant\|A R-R A\|$ для любого самосопряжённого оператоpa $A$ и любого ограниченного линейного оператора $R$;

(с) $\|f(A) R-R f(B)\| \leqslant\|A R-R B\|$ для произвольных самосопряжённых операторов $A$ и $B$ и для любого ограниченного линейного оператора $R$.

Операторы вида $f(A) R-R f(B)$ называются квазикоммутаторами. 
Мы выведем теорему 1.3.1 из более общего результата для функций нормальных операторов в разделе 3.1. Заметим, однако, что в случае функций нормальных операторов коммутаторная липшицевость отнюдь не эквивалентна операторной липшицевости.

\section{4. Операторные неравенства Бернштейна}

В этом разделе мы приводим элементарное доказательство результата работы [58] о том, что функции из $L^{\infty}(\mathbb{R})$, преобразование Фурье которых имеет компактный носитель, операторно липшицевы. Более того, мы получим так называемое операторное неравенство Бернштейна с константой 1. Мы следуем подходу работы [10]. Мы также получим аналогичные результаты для функций на окружности.

Отметим, что в разделе 1.6 мы увидим из этих результатов, что достаточным условием для операторной липшицевости является принадлежность классу Бесова $B_{\infty, 1}^{1}(\mathbb{R})$.

Пусть $\sigma>0$. Напомним, что целая функция $f$ называется функцией экспоненииального типа не выше $\sigma$, если для любого положительного числа $\varepsilon$ найдётся число $c>0$ такое, что $|f(z)| \leqslant c e^{(\sigma+\varepsilon)|z|}$ при всех $z \in \mathbb{C}$.

Обозначим через $\mathscr{E}_{\sigma}$ множество всех целых функций экспоненциального типа не выше $\sigma$. Хорошо известно, что

$$
\mathscr{E}_{\sigma} \cap L^{\infty}(\mathbb{R})=\left\{f \in L^{\infty}(\mathbb{R}): \operatorname{supp} \mathscr{F} f \subset[-\sigma, \sigma]\right\} .
$$

Отметим ещё, что пространство $\mathscr{E}_{\sigma} \cap L^{\infty}(\mathbb{R})$ совпадает с множеством всех целых функций $f$ таких, что $f \in L^{\infty}(\mathbb{R})$ и

$$
|f(z)| \leqslant e^{\sigma|\operatorname{Im} z|}\|f\|_{L^{\infty}(\mathbb{R})}, \quad z \in \mathbb{C}
$$

(см., например, [42; с. 97]).

Неравенство Бернштейна (см. [18]) утверждает, что

$$
\sup _{x \in \mathbb{R}}\left|f^{\prime}(x)\right| \leqslant \sigma \sup _{x \in \mathbb{R}}|f(x)|
$$

для любой функции $f$ из $\mathscr{E}_{\sigma} \cap L^{\infty}(\mathbb{R})$. Отсюда вытекает, что

$$
|f(x)-f(y)| \leqslant \sigma\|f\|_{L^{\infty}(\mathbb{R})}|x-y|, \quad f \in \mathscr{E}_{\sigma} \cap L^{\infty}(\mathbb{R}), \quad x, y \in \mathbb{R},
$$

где $\|f\|_{L^{\infty}(\mathbb{R})} \stackrel{\text { def }}{=} \sup _{x \in \mathbb{R}}|f(x)|$.

Бернштейн также доказал в [18] следующее усиление неравенства (1.4.2):

$$
|f(x)-f(y)| \leqslant \beta(\sigma(|x-y|))\|f\|_{L^{\infty}(\mathbb{R})}, \quad f \in \mathscr{E}_{\sigma} \cap L^{\infty}(\mathbb{R}), \quad x, y \in \mathbb{R},
$$

где

$$
\beta(t) \stackrel{\text { def }}{=} \begin{cases}2 \sin (t / 2), & \text { если } 0 \leqslant t \leqslant \pi, \\ 2, & \text { если } t>\pi .\end{cases}
$$

Заметим, что $\beta(t) \leqslant \min \{t, 2\}$ при всех $t \geqslant 0$.

Пусть $X$ - комплексное банахово пространство. Обозначим через $\mathscr{E}_{\sigma}(X)$ пространство всех целых $X$-значных функций $f$ экспоненциального типа не выше $\sigma$, т. е. удовлетворяющих следующему условию: для любого числа $\varepsilon>0$ найдётся число $c>0$ такое, что $\|f(z)\|_{X} \leqslant c e^{(\sigma+\varepsilon)|z|}$ при всех $z \in \mathbb{C}$. 
Неравенство Бернштейна для векторнозначных функций. Пусть $f-$ функиия из $\mathscr{E}_{\sigma}(X) \cap L^{\infty}(\mathbb{R}, X)$, где $\sigma>0$. Тогда

$$
\|f(x)-f(y)\|_{X} \leqslant \beta(\sigma(|x-y|))\|f\|_{L^{\infty}(\mathbb{R}, X)} \leqslant \sigma\|f\|_{L^{\infty}(\mathbb{R}, X)}|x-y|
$$

для всех $x, y \in \mathbb{R}$.

Векторная версия неравенства Бернштейна сводится к скалярной версии при помощи теоремы Хана-Банаха.

Tеорема 1.4.1. Пусть $f \in \mathscr{E}_{\sigma} \cap L^{\infty}(\mathbb{R})$. Тогда

$$
\|f(A)-f(B)\| \leqslant \beta(\sigma(\|A-B\|))\|f\|_{L^{\infty}} \leqslant \sigma\|f\|_{L^{\infty}}\|A-B\|
$$

для любых (ограниченных) самосопряжённых операторов $A$ и B. $B$ частно$c m u,\|f\|_{\mathrm{OL}(\mathbb{R})} \leqslant \sigma\|f\|_{L^{\infty}(\mathbb{R})}$.

ДокАЗАТЕЛЬСтво теОРемы 1.4.1. Пусть $A$ и $B$ - самосопряжённые операторы в гильбертовом пространстве $\mathscr{H}$. Нам нужно доказать, что

$$
\|f(A)-f(B)\| \leqslant \beta(\sigma\|A-B\|)\|f\|_{L^{\infty}} .
$$

Положим $F(z)=f(A+z(B-A))$. Ясно, что $F$ - целая функция со значениями в пространстве операторов $\mathcal{B}(\mathscr{H})$ и $\|F(t)\| \leqslant\|f\|_{L^{\infty}(\mathbb{R})}$ для всех $t \in \mathbb{R}$. Из неравенства фон Неймана (см. [72]) следует, что $F \in \mathscr{E}_{\sigma\|B-A\|}(\mathcal{B}(\mathscr{H}))$. Чтобы закончить доказательство, остаётся применить неравенство Бернштейна (1.4.4) к векторнозначной функции $F$ при $x=0$ и $y=1$. Теорема доказана.

Ранее в работе [58] было доказано, что

$$
\|f\|_{\mathrm{OL}(\mathbb{R})} \leqslant \text { const } \sigma\|f\|_{L^{\infty}(\mathbb{R})}, \quad f \in \mathscr{E}_{\sigma} \cap L^{\infty}(\mathbb{R}),
$$

и, в частности, $\mathscr{E}_{\sigma} \cap L^{\infty}(\mathbb{R}) \subset \mathrm{OL}(\mathbb{R})$. Отсюда следует, что для любой функции $f \in \mathscr{E}_{\sigma} \cap \operatorname{Lip}(\mathbb{R})$ функция $f^{\prime}$ операторно липшицева.

Следующий пример показывает, что $\mathscr{E}_{\sigma} \cap \operatorname{Lip}(\mathbb{R}) \not \subset \mathrm{OL}(\mathbb{R})$. Пример. Рассмотрим функцию $f(x) \stackrel{\text { def }}{=} \int_{0}^{x} \mathrm{Si}(t) d t$, где $\mathrm{Si}$ обозначает ин-
тегральный синус:

$$
\operatorname{Si}(x) \stackrel{\text { def }}{=} \int_{0}^{x} \frac{\sin t}{t} d t
$$

Ясно, что $f \in \mathscr{E}_{1} \cap \operatorname{Lip}(\mathbb{R})$, но функиия $f$ не может быть операторно липшицевой (см. теоремы 3.3 .2 и 3.3 .3 ниже), поскольку не существует предела $\lim _{|x| \rightarrow \infty} x^{-1} f(x)$ (на самом деле $\lim _{x \rightarrow \infty} x^{-1} f(x)=\lim _{x \rightarrow \infty} \operatorname{Si}(x)=\pi / 2=$ $\left.-\lim _{x \rightarrow-\infty} x^{-1} f(x)\right)$.

Интересно отметить, что если функцию $f$ из этого примера чуть “испортить", заменив её функцией $g(x) \stackrel{\text { def }}{=} \int_{0}^{x} \operatorname{Si}(|t|) d t$, то она станет операторно липшицевой. Достаточно убедиться в том, что функция $g(x)-\pi x / 2$ является операторно липшицевой. Это следует из того (см. [21; предложение 7.8]), что производная этой функции принадлежит пространству $L^{2}(\mathbb{R}) \cap \operatorname{Lip}(\mathbb{R})$ (это также можно вывести из теоремы 1.6.4 ниже).

Установим аналоги неравенств Бернштейна для унитарных операторов. 
Лемма 1.4.2. Пусть $U$ и $V$ - унитарные операторъ. Тогда существует самосопряжённый оператор $A$ такой, что $V=e^{\mathrm{i} A} U,\|A\| \leqslant \pi u \beta(\|A\|)=$ $\|U-V\|$.

ДокАЗАтельство. Определим оператор $A$ равенством $A=\arg \left(V U^{-1}\right)$, где функция $\arg$ определена на окружности $\mathbb{T}$ равенством $\arg \left(e^{\mathrm{i} s}\right)=s, s \in[-\pi, \pi)$. Очевидно, что $\beta(\|A\|)=\left\|I-e^{\mathrm{i} A}\right\|=\|U-V\|$. Лемма 1.4.2 доказана.

Теорема 1.4.3. Пусть $f$ - тригонометрический полином степени не более $n$. Тогда для любых унитарных операторов $U u V$

$$
\|f(U)-f(V)\| \leqslant n\|f\|_{L^{\infty}(\mathbb{T})}\|U-V\| .
$$

ДокАзАтельство. Пусть $A$ - самосопряжённый оператор, существование которого утверждается в лемме 1.4.2. Положим $\Phi(z) \stackrel{\text { def }}{=} f\left(e^{\mathrm{i} z A} U\right), z \in \mathbb{C}$, где тем же символом $f$ мы обозначаем аналитическое продолжение в $\mathbb{C} \backslash\{0\}$ тригонометрического полинома $f$. Ясно, что $\Phi$ - целая функция со значениями в $\mathcal{B}(\mathscr{H})$ и $\|\Phi(t)\| \leqslant\|f\|_{L^{\infty}(\mathbb{T})}$ для всех $t \in \mathbb{R}$. Из неравенства фон Неймана (см. [72]) следует, что $\Phi \in \mathscr{E}_{n\|A\|}(\mathcal{B}(\mathscr{H}))$. Применяя векторное неравенство Бернштейна, получаем:

$$
\|f(U)-f(V)\|=\|\Phi(1)-\Phi(0)\| \leqslant \beta(n\|A\|)\|f\|_{L^{\infty}(\mathbb{T})} .
$$

Остаётся заметить, что $\beta(n\|A\|) \leqslant n \beta(\|A\|)=n\|U-V\|$. Теорема доказана.

Отметим, что в [56] доказано, что $\|f(U)-f(V)\| \leqslant$ const $n\|f\|_{L^{\infty}(\mathbb{T})}\|U-V\|$ для любого тригонометрического полинома $f$ степени $n$ и для любых унитарных операторов $U$ и $V$.

ЗАмечАниЕ. Из доказательства теоремы 1.4.3 видно, что

$$
\|f(U)-f(V)\| \leqslant \beta(n\|A\|)\|f\|_{L^{\infty}(\mathbb{T})}=\beta\left(2 n \arcsin \frac{\|U-V\|}{2}\right)\|f\|_{L^{\infty}(\mathbb{T})} .
$$

Эта оценка неулучшаема для функции $f(z)=z^{n}$, поскольку $\sup \left\{\left|z_{1}^{n}-z_{2}^{n}\right|: z_{1} \in \mathbb{T}\right.$, $\left.z_{2} \in \mathbb{T},\left|z_{1}-z_{2}\right|<n\right\}=\beta(2 n \arcsin (\delta / 2)), \delta \in(0,2]$.

\section{5. Необходимые условия для операторной липшицевости}

В этом разделе для функций на прямой и на окружности мы получим необходимые условия для операторной липшицевости. Эти необходимые условия по существу были получены в работах [56] и [58], в которых использовались другие методы. В этом разделе для достижения цели помимо критерия ядерности операторов Ганкеля (см. п. 3 раздела 2), на который также опираются работы [56] и [58], используются и результаты раздела 3.12 настоящего обзора о поведении производных операторно липшицевых функций при дробно-линейных преобразованиях.

Для доказательства следующего результата мы воспользуемся результатами раздела 3.6 о поведении функций от операторов при ядерных возмущениях.

Теорема 1.5.1. Пусть $f$ - операторно липшицева функиия на окружности T. Тогда $f \in B_{1}^{1}(\mathbb{T})$. 
ДокАзАтельство. Ввиду замечания к теореме 3.6.5, функция $f$ обладает следующим свойством: $f(U)-f(V) \in \boldsymbol{S}_{1}$, как только $U$ и $V$ - унитарные операторы такие, что $U-V \in \boldsymbol{S}_{1}$.

Определим операторы $U$ и $V$ в пространстве $L^{2}(\mathbb{T})$ равенствами

$$
U f=\bar{z} f, \quad V f=\bar{z} f-2(f, \mathbf{1}) \bar{z}, \quad f \in L^{2} .
$$

Легко видеть, что $U$ и $V-$ унитарные операторы и $\operatorname{rank}(V-U)=1$. Также нетрудно проверить, что при $n \geqslant 0$ имеет место равенство

$$
V^{n} z^{j}= \begin{cases}z^{j-n}, & j \geqslant n \text { или } j<0, \\ -z^{j-n}, & 0 \leqslant j<n .\end{cases}
$$

Отсюда получаем, что для любой непрерывной функции $f$ на $\mathbb{T}$ справедливо соотношение

$$
\begin{aligned}
&\left((f(V)-f(U)) z^{j}, z^{k}\right)= \sum_{n>0} \widehat{f}(n)\left(\left(V^{n} z^{j}, z^{k}\right)-\left(z^{j-n}, z^{k}\right)\right) \\
&+\sum_{n<0} \widehat{f}(n)\left(\left(V^{n} z^{j}, z^{k}\right)-\left(z^{j-n}, z^{k}\right)\right) \\
&=-2 \begin{cases}\widehat{f}(j-k), & j \geqslant 0, k<0, \\
\widehat{f}(j-k), & j<0, k \geqslant 0, \\
0 & \text { в противном случае. }\end{cases}
\end{aligned}
$$

Таким образом, если $f(U)-f(V) \in \boldsymbol{S}_{1}$, то операторы в $\ell^{2}$ с ганкелевыми матрицами $\{\widehat{f}(j+k)\}_{j \geqslant 0, k \geqslant 1}$ и $\{\widehat{f}(-j-k)\}_{j \geqslant 0, k \geqslant 1}$ входят в класс $\boldsymbol{S}_{1}$. Теперь можно воспользоваться критерием ядерности операторов Ганкеля (см. п. 3 раздела 2) и заключить, что $f \in B_{1}^{1}(\mathbb{T})$. Теорема доказана.

Отметим, что конструкция в доказательстве теоремы 1.5.1 заимствована из [9]. Нам удобно в этом разделе ввести обозначение $\|M\|$ для нормы матрицы $M$.

Чтобы сформулировать следствие теоремы 1.5.1, нам понадобится банахово пространство $(\mathrm{OL})_{\mathrm{loc}}^{\prime}(\mathbb{T})$, которое будет подробно рассмотрено в разделе 3.12 . Здесь мы только отметим, что $(\mathrm{OL})_{\text {lос }}^{\prime}(\mathbb{T})=\left\{f^{\prime}+c \bar{z}: f \in \mathrm{OL}(\mathbb{T}), c \in \mathbb{C}\right\}$ (см. следствие 3.12.6), причём, как всегда в этой статье, производная понимается в комплексном смысле, т. е. $f^{\prime}(\zeta) \stackrel{\text { def }}{=} \lim _{\tau \rightarrow \zeta}(\tau-\zeta)^{-1}(f(\tau)-f(\zeta))$.

СлеДСтвиЕ 1.5.2. Пусть и-интеграл Пуассона функиии $f \in(\mathrm{OL})_{\mathrm{loc}}^{\prime}(\mathbb{T})$. Тогда $\|\nabla u\| \in L^{1}(\mathbb{D}) u\|\| \nabla u\|\|_{L^{1}(\mathbb{D})} \leqslant$ const $\cdot\|f\|_{(\mathrm{OL})_{\text {loc }}^{\prime}(\mathbb{T})} \cdot$

ДоказАтельство. Пусть $f=g^{\prime}$, где $g \in \mathrm{OL}(\mathbb{T})$. Тогда $f \in B_{1}^{0}(\mathbb{T})$, поскольку $g \in B_{1}^{1}(\mathbb{T})$, и достаточно воспользоваться характеризацией класса Бесова $B_{1}^{0}(\mathbb{T})$ в терминах гармонического продолжения (см. раздел 2). Остаётся заметить, что следствие очевидно для функции $f(z)=z^{-1}=\bar{z}$.

Чтобы сформулировать более сильное необходимое условие для операторной липшицевости, нам понадобится понятие меры Карлесона. Пусть $\mu$ - положительная борелевская мера в открытом единичном круге $\mathbb{D}$. Хорошо известная теорема Карлесона утверждает, что класс Харди $H^{p}$ содержится в $L^{p}(\mu)$ 
$(0<p<+\infty)$ в том и только том случае, когда для любой точки $\zeta$ единичной окружности $\mathbb{T}$ и любого положительного числа $r$ выполняется неравенство

$$
\mu\{z \in \mathbb{D}:|z-\zeta|<r\} \leqslant \text { const } \cdot r .
$$

Такие меры $\mu$ называются мерами Карлесона в круге $\mathbb{D}$. Заметим, что условие Карлесона не зависит от $p \in(0,+\infty)$. Подробнее о мерах Карлесона см., например, [50], [51]. Нам понадобится следующая эквивалентная переформулировка условия Карлесона:

$$
\sup _{a \in \mathbb{D}} \int_{\mathbb{D}} \frac{1-|a|^{2}}{|1-\bar{a} z|^{2}} d \mu(z)<+\infty
$$

(см., например, [50; лекция VII]). Заметим, что условие (1.5.1) означает, что $\left\|k_{a}\right\|_{L^{2}(\mu)} \leqslant$ const $\left\|k_{a}\right\|_{H^{2}}$ для всех $a \in \mathbb{D}$, где $k_{a}(z) \stackrel{\text { def }}{=}(1-z \bar{a})^{-1}-$ воспроизводящее ядро гильбертова пространства $H^{2}$.

Обозначим символом $\mathrm{CM}(\mathbb{D})$ пространство всех комплексных мер Радона $\mu$ в $\mathbb{D}$ таких, что $|\mu|$ - мера Карлесона, и символом $\|\mu\|_{\mathrm{CM}(\mathbb{D})}$ норму тождественного оператора вложения из $H^{1}$ в $L^{1}(|\mu|)$. Хорошо известно, что (квази)норма тождественного оператора вложения из $H^{p}$ в $L^{p}(|\mu|)$ равна $\|\mu\|_{\mathrm{CM}(\mathbb{D})}^{1 / p}$ при всех $p \in(0,+\infty)$.

Всё сказанное выше о мерах Карлесона в круге $\mathbb{D}$ имеет естественные аналоги для полуплоскости $\mathbb{C}_{+}$. В этом случае условие Карлесона для положительной борелевской меры $\mu$ в $\mathbb{C}_{+}$переписывается следующим образом:

$$
\mu\left\{z \in \mathbb{C}_{+}:|z-t|<r\right\} \leqslant \text { const } \cdot r
$$

для всех $t \in \mathbb{R}$ и всех $r>0$. Аналогом условия (1.5.1) является неравенство

$$
\sup _{a \in \mathbb{C}_{+}} \int_{\mathbb{C}_{+}} \frac{\operatorname{Im} a}{|z-\bar{a}|^{2}} d \mu(z)<\infty .
$$

В частности, точно так же можно ввести пространство $\mathrm{CM}\left(\mathbb{C}_{+}\right)$и норму в нём.

Пусть $f-$ (обобщённая) функция на единичной окружности $\mathbb{T}$. Обозначим символом $\mathcal{P} f$ интеграл Пуассона функиии $f$.

Teорема 1.5.3. Пусть $f \in(\mathrm{OL})_{\mathrm{loc}}^{\prime}(\mathbb{T})$. Тогда $\|\nabla(\mathcal{P} f)\| d \boldsymbol{m}_{2} \in \mathrm{CM}(\mathbb{D})$.

ДоказАТЕльство. Пусть $f \in(\mathrm{OL})_{\mathrm{loc}}^{\prime}(\mathbb{T})$. Тогда из теоремы 3.12 .10 и следствия 1.5.2 вытекает, что

$$
\int_{\mathbb{D}}\|((\nabla u) \circ \varphi)(z)\| \cdot\left|\varphi^{\prime}(z)\right| d \boldsymbol{m}_{2} \leqslant \text { const }\|f\|_{(\mathrm{OL})_{\mathrm{loc}}^{\prime}(\mathbb{T})}
$$

для любого дробно-линейного автоморфизма единичного круга $\mathbb{D}$, где $u=\mathcal{P} f$. Возьмём в качестве $\varphi$ функцию $\varphi(z)=(1-\bar{a} z)^{-1}(a-z)$, где $a \in \mathbb{D}$. Делая в интеграле (1.5.2) замену переменной $z=\varphi(w)$, получаем

$$
\sup _{a \in \mathbb{D}} \int_{\mathbb{D}}\|(\nabla u)(w)\| \frac{1-|a|^{2}}{|1-\bar{a} w|^{2}} d \boldsymbol{m}_{2}(w) \leqslant \text { const }\|f\|_{(\mathrm{OL})^{\prime}(\mathbb{T})} .
$$

Таким образом, мера $\|\nabla u\| d \boldsymbol{m}_{2}=\|\nabla(\mathcal{P} f)\| d \boldsymbol{m}_{2}$ удовлетворяет условию (1.5.1). Теорема доказана. 
Следующий результат является переформулировкой теоремы 1.5.3.

Teоpema 1.5.4. Пусть $f \in \mathrm{OL}(\mathbb{T})$. Тогда $a^{2}\|\operatorname{Hess} \mathcal{P} f\| d \boldsymbol{m}_{2} \in \mathrm{CM}(\mathbb{D})$.

Перейдём к интегралам Пуассона функций на $\mathbb{R}$. Если $f$ принадлежит пространству $L^{1}\left(\mathbb{R},\left(1+x^{2}\right)^{-1} d x\right)$, то интеграл Пуассона определяется стандартным образом. Нам понадобится интеграл Пуассона функции $f$ такой, что $f^{\prime} \in L^{1}\left(\mathbb{R},\left(1+x^{2}\right)^{-1} d x\right)$. Ясно, что достаточно рассмотреть случай вещественной функции $f$. Пусть $u$ - интеграл Пуассона функции $f^{\prime}$. Обозначим буквой $v$ гармоническую функцию, сопряжённую с $u$. Функция $u+\mathrm{i} v$ имеет первообразную $F$ такую, что граничные значения функции $\operatorname{Re} F$ совпадают с $f$ всюду на $\mathbb{R}$. Функция $F$ не определяется однозначно, поскольку не определена однозначно гармонически сопряжённая функция $v$. Семейство $\{v+c\}_{c \in \mathbb{R}}$ состоит из всех гармонических функций, сопряжённых с функцией $u$. Нужная нам первообразная функции $u+\mathrm{i}(v+c)$ имеет вид $F+c \mathrm{i} z+\mathrm{i} \alpha$, где $\alpha \in \mathbb{R}$. Заметим, что $\operatorname{Re}(F+c \mathrm{i} z+\mathrm{i} \alpha)=\operatorname{Re} F-c y$. Таким образом, естественно определить интеграл Пуассона функции $f$ как класс функций $\{\operatorname{Re} F-c y\}_{c \in \mathbb{R}}$. Поскольку Hess $y=0$, гессиан интеграла Пуассона Hess $\mathcal{P} f$ функции $f$ определяется однозначно.

В следующем утверждении $(\mathrm{OL})^{\prime}(\mathbb{R}) \stackrel{\text { def }}{=}\left\{f^{\prime}: f \in \mathrm{OL}(\mathbb{R})\right\}$.

TeOpema 1.5.5. Пусть $f \in(\mathrm{OL})^{\prime}(\mathbb{R})$. Тогда $\|\nabla \mathcal{P} f\| d \boldsymbol{m}_{2} \in \mathrm{CM}\left(\mathbb{C}_{+}\right)$.

ДокАЗАТЕЛЬСтво. Пусть $f \in(\mathrm{OL})^{\prime}(\mathbb{R})$. Тогда из теоремы 3.12 .9 и следствия 1.5.2 вытекает, что

$$
\int_{\mathbb{D}}\|((\nabla u) \circ \varphi)(z)\| \cdot\left|\varphi^{\prime}(z)\right| d \boldsymbol{m}_{2} \leqslant \text { const }\|f\|_{(\mathrm{OL})^{\prime}(\mathbb{R})}
$$

для любого автоморфизма $\varphi$ из $\operatorname{Aut}(\widehat{\mathbb{C}})$ такого, что $\varphi(\mathbb{D})=\mathbb{C}_{+}$, где $u=\mathcal{P} f$. Возьмём в качестве $\varphi$ функцию $\varphi(z)=(1-z)^{-1}(a-\bar{a} z)$, где $a \in \mathbb{C}_{+}$. Делая в интеграле (1.5.2) замену переменной $z=(w-\bar{a})^{-1}(w-a)$, получаем

$$
\sup _{a \in \mathbb{C}_{+}} \int_{\mathbb{C}_{+}}\|(\nabla u)(w)\| \frac{2 \operatorname{Im} a}{|w-\bar{a}|^{2}} d \boldsymbol{m}_{2}(w) \leqslant \mathrm{const}\|f\|_{(\mathrm{OL})_{\mathrm{loc}}^{\prime}(\mathbb{T})} .
$$

Последнее условие равносильно карлесоновости меры $\|\nabla u\| d \boldsymbol{m}_{2}$. Теорема доказана.

Teоpema 1.5.6. Пусть $f \in \mathrm{OL}(\mathbb{R})$. Тогда $\|\operatorname{Hess} \mathcal{P} f\| d \boldsymbol{m}_{2} \in \mathrm{CM}\left(\mathbb{C}_{+}\right)$.

Необходимые условия операторной липшицевости, приведённые выше, были получены первоначально в работах [56] и [58]. Именно, в работе [56] было доказано, что если $f \in \mathrm{OL}(\mathbb{T})$, то оба оператора Ганкеля $H_{f}$ и $H_{\bar{f}}$ отображают пространство Харди $H^{1}$ в класс Бесова $B_{1}^{1}(\mathbb{T})$ (класс таких функций $f$ обозначен в [56] символом $L$ ). С. Семмес заметил, что $f \in L$ тогда и только тогда, когда мера | Hess $\mathcal{P} f \| d \boldsymbol{m}_{2}$ является карлесоновой; см. [59], где изложено доказательство этой эквивалентности. Аналогичное утверждение имеет место и для функций на $\mathbb{R}$ (см. [58]). В работе [56] также показано, что необходимое условие для операторной липшицевости, обсуждаемое выше, не является достаточным. Более того, оно не является достаточным даже для липшицевости.

\footnotetext{
23десь и далее Hess обозначает гессиан, т. е. матрицу из частных производных второго порядка.
} 
Рассмотрим теперь пространства $\mathbb{P}_{+}\left(b_{\infty}^{-1}(\mathbb{T})\right)$ и $\mathbb{P}_{+}\left(b_{1, \infty}^{-1}(\mathbb{T})\right)$, которые являются замыканиями множества аналитических полиномов в пространствах Бесова $B_{\infty}^{-1}(\mathbb{T})$ и $B_{1, \infty}^{-1}(\mathbb{T})$. Хорошо известно, что эти пространства допускают следующие описания в терминах аналитического продолжения в единичный круг:

$$
\begin{aligned}
\mathbb{P}_{+}\left(b_{\infty}^{-1}(\mathbb{T})\right) & =\left\{h: \lim _{r \rightarrow 1^{-}}(1-r)\|h(r z)\|_{L^{\infty}(\mathbb{T})}=0\right\} \\
\mathbb{P}_{+}\left(b_{1, \infty}^{-1}(\mathbb{T})\right) & =\left\{h: \lim _{r \rightarrow 1^{-}}(1-r)\|h(r z)\|_{L^{1}(\mathbb{T})}=0\right\}
\end{aligned}
$$

В [56] также отмечено, что пространство $\mathbb{P}_{+} L$ является двойственным к пространству аналитических функций $g$ в круге $\mathbb{D}$, допускающих представление

$$
\begin{aligned}
g=\sum_{n} \varphi_{n} \psi_{n}, \quad \text { где } \quad \varphi_{n} \in H^{1}, \quad \psi_{n} \in \mathbb{P}_{+}\left(b_{\infty}^{-1}(\mathbb{T})\right), \\
\\
\sum_{n}\left\|\varphi_{n}\right\|_{H^{1}}\left\|\psi_{n}\right\|_{\mathbb{P}_{+}\left(b_{\infty}^{-1}(\mathbb{T})\right)}<\infty .
\end{aligned}
$$

Очевидно, что такие функции $g$ входят в пространство $\mathbb{P}_{+}\left(b_{1, \infty}^{-1}(\mathbb{T})\right)$, двойственное к которому отождествляется естественным образом с пространством Бесова $\mathbb{P}_{+} B_{\infty, 1}^{1}(\mathbb{T})$ аналитических в $\mathbb{D}$ функций. Тем не менее не всякая функция из $\mathbb{P}_{+}\left(b_{1, \infty}^{-1}(\mathbb{T})\right)$ представима в виде (1.5.4). Иначе мы имели бы равенство $L=B_{\infty, 1}^{1}(\mathbb{T})$, что невозможно, ибо условие $f \in L$, будучи необходимым для операторной липшицевости, не является достаточным.

ЗАмЕчАНИЕ. Отметим здесь, что пространство

$$
L=\left\{f \in \operatorname{BMO}(\mathbb{R}):\|\operatorname{Hess} \mathcal{P} f\| d \boldsymbol{m}_{2} \in \mathrm{CM}\left(\mathbb{C}_{+}\right)\right\}
$$

является предельным пространством шкалы пространств Трибеля-Лизоркина $F_{p, q}^{s}(\mathbb{R})$ и обозначается символом $F_{\infty, 1}^{1}(\mathbb{R})$ (см. $\left.[27 ; \S 5]\right)$. Аналогичным образом определяется пространство Трибеля-Лизоркина $F_{\infty, 1}^{1}(\mathbb{T})$ функций на $\mathbb{T}$. Полученные здесь необходимые условия операторной липшицевости можно переформулировать следующим образом: $\mathrm{OL}(\mathbb{R}) \subset F_{\infty, 1}^{1}(\mathbb{R})$ и $\mathrm{OL}(\mathbb{T}) \subset F_{\infty, 1}^{1}(\mathbb{T})$.

\section{6. Достаточное условие для операторной липшицевости в терминах классов Бесова}

В этом разделе мы покажем, что функции класса Бесова $B_{\infty, 1}^{1}(\mathbb{R})$ (см. раздел 2) являются операторно липшицевыми. Мы также получим аналогичный результат для функций на окружности. Приводимые здесь доказательства отличаются от оригинальных доказательств работ [56] и [58] и основаны на операторных неравенствах Бернштейна (см. раздел 1.4).

Теорема 1.6.1. Пусть $f \in B_{\infty, 1}^{1}(\mathbb{R})$. Тогда функиия $f$ операторно липиицева и

$$
\|f(A)-f(B)\| \leqslant \text { const }\|f\|_{B_{\infty, 1}^{1}}\|A-B\|
$$

для любых самосопряжённых операторов $A, B$ с ограниченной разностью $A-B$. 
ДокАзАтЕльство. Как мы отмечали во введении (см. теорему 3.2.1 ниже), достаточно доказать (1.6.1) для ограниченных самосопряжённых операторов.

Не умаляя общности, мы можем считать, что $f(0)=0$. Рассмотрим функции $f_{n}=f * W_{n}$, определённые равенством (2.2). Пусть $N \in \mathbb{Z}$. Положим $g_{n} \stackrel{\text { def }}{=} f_{n}-f_{n}(0)$. Из определения класса $B_{\infty, 1}^{1}(\mathbb{R})$ (см. раздел 2) вытекает, что $\sum_{n=-\infty}^{\infty} g_{n}^{\prime}=f^{\prime}$, причём ряд сходится равномерно на $\mathbb{R}$. Поэтому ряд $\sum_{n=-\infty}^{\infty} g_{n}$ сходится равномерно на каждом компактном подмножестве в $\mathbb{R}$. Стало быть,

$$
\sum_{n=-\infty}^{\infty} g_{n}(A)=f(A) \quad \text { и } \quad \sum_{n=-\infty}^{\infty} g_{n}(B)=f(B),
$$

причём ряды сходятся абсолютно по операторной норме.

Поскольку очевидно, что $f_{n} \in \mathscr{E}_{2^{n}+1} \cap L^{\infty}(\mathbb{R})$, операторное неравенство Бернштейна (1.4.5) позволяет заключить, что

$$
\begin{aligned}
\|f(A)-f(B)\| & \leqslant\left\|\sum_{n=-\infty}^{\infty}\left(g_{n}(A)-g_{n}(B)\right)\right\|=\left\|\sum_{n=-\infty}^{\infty}\left(f_{n}(A)-f_{n}(B)\right)\right\| \\
& \leqslant \sum_{n=-\infty}^{\infty} 2^{n+1}\left\|f_{n}\right\|_{L^{\infty}}\|A-B\| \leqslant \text { const }\|f\|_{B_{\infty, 1}^{1}}\|A-B\| .
\end{aligned}
$$

Теорема доказана.

Аналогичным образом можно доказать следующий аналог теоремы 1.6.1 для функций на единичной окружности.

Tеорема 1.6.2. Пустъ $f \in B_{\infty, 1}^{1}(\mathbb{T})$. Тогда функиия $f$ операторно липиииева и

$$
\|f(U)-f(V)\| \leqslant \text { const }\|f\|_{B_{\infty, 1}^{1}}\|U-V\|, \quad f \in B_{\infty, 1}^{1}(\mathbb{T}),
$$

для любых унитарных операторов $U$ u $V$.

Следующая формулировка позволяет нам объединить необходимые условия, полученные в разделе 1.5 , с достаточными условиями настоящего раздела.

TEOPEMA 1.6.3. $B_{\infty, 1}^{1}(\mathbb{T}) \subset \mathrm{OL}(\mathbb{T}) \subset F_{\infty, 1}^{1}(\mathbb{T}) u B_{\infty, 1}^{1}(\mathbb{R}) \subset \mathrm{OL}(\mathbb{T}) \subset F_{\infty, 1}^{1}(\mathbb{R})$.

Оказывается, что функции класса $B_{\infty, 1}^{1}(\mathbb{R})$ не только операторно липшицевы, но и операторно дифференцируемы.

Теорема 1.6.4. Пусть $f \in B_{\infty, 1}^{1}(\mathbb{R})$. Тогда $f$ операторно дифферениируема.

Мы отсылаем читателя к работам [58] и [61] за доказательством теоремы 1.6.4.

\section{7. Операторно гёльдеровы функции}

В этом разделе мы обсудим другие приложения операторных неравенств Бернштейна, полученных в разделе 1.4. Мы покажем, что класс операторно гёльдеровых функций порядка $\alpha, 0<\alpha<1$, совпадает с классом гёльдеровых функций порядка $\alpha$. Мы также кратко остановимся на случае произвольных модулей непрерывности. Результаты этого раздела получены в [7] и [8]. Другой 
подход к этим задачам найден в [49], где получены аналогичные результаты для функций класса Гёльдера с несколько худшими константами и несколько более слабый результат для произвольных модулей непрерывности.

Хорошо известно, что (скалярное) неравенство Бернштейна играет важную роль в теории приближений (см., например, [1], [24], [47], [73]). Речь идёт об описании свойств типа гладкости в терминах приближений хорошими функциями. Прямые теоремы теории приближений дают оценки приближений функций из данного функционального пространства $X$ (обычно в том или ином смысле гладких функций) хорошими функциями. Обратные теоремы позволяют по оценкам приближений данной функции $f$ хорошими функциями сделать вывод о принадлежности данной функции тому или иному функциональному пространству. В случае, когда для функционального пространства $X$ прямые теоремы “стыкуются" с обратными теоремами, получается полное описание пространства $X$ в терминах приближений.

В этом разделе мы будем рассматривать функциональные пространства на единичной окружности $\mathbb{T}$ и на вещественной прямой $\mathbb{R}$. В качестве хороших функций в первом случае берутся пространства $\mathcal{P}_{n}$ тригонометрических многочленов степени не выше $n$, а во втором - пространства целых функций $\mathscr{E}_{\sigma}$ экспоненциального типа не выше $\sigma$. Мы будем рассматривать только равномерные приближения.

Классические неравенства Бернштейна играют решающую роль при доказательстве обратных теорем теории приближений. Нетрудно понять, что если в таком доказательстве воспользоваться операторной версией неравенства Бернштейна, то мы получим соответствующую гладкость функции $f$ на множестве унитарных операторов (если речь идёт о функциях на окружности) или на множестве самосопряжённых операторов (если речь идёт о функциях на прямой).

Проиллюстрируем это на примере. Классическая теорема Джексона утверждает, что если функция $f$ входит в класс Гёльдера $\Lambda_{\alpha}(\mathbb{T}), 0<\alpha<1$, то

$$
\operatorname{dist}\left(f, \mathcal{P}_{n}\right) \leqslant \operatorname{const}(n+1)^{-\alpha}\|f\|_{\Lambda_{\alpha}} .
$$

С. Н. Бернштейн доказал, что верно и обратное, т. е. если для некоторой функции $f$ из $C(\mathbb{T})$ при $\alpha \in(0,1)$ выполняются неравенства $(1.7 .1)$, то $f \in \Lambda_{\alpha}(\mathbb{T})$.

Приведём стандартное доказательство этого результата Бернштейна. Не умаляя общности, можно считать, что $c=1$. Для $n \geqslant 0$ найдётся тригонометрический полином $f_{n}$ такой, что $\operatorname{deg} f_{n}<2^{n}$ и $\left\|f-f_{n}\right\|_{C(\mathbb{T})} \leqslant 2^{-\alpha n}$. Ясно, что

$$
\left\|f_{n}-f_{n-1}\right\|_{C(\mathbb{T})} \leqslant\left\|f-f_{n}\right\|_{C(\mathbb{T})}+\left\|f-f_{n-1}\right\|_{C(\mathbb{T})} \leqslant 2^{-\alpha n}\left(1+2^{\alpha}\right) \leqslant 3 \cdot 2^{-\alpha n} .
$$

Следовательно,

$$
\left\|f_{n}-f_{n-1}\right\|_{\operatorname{Lip}(\mathbb{T})} \leqslant 2^{n}\left\|f_{n}-f_{n-1}\right\|_{C(\mathbb{T})} \leqslant 3 \cdot 2^{(1-\alpha) n}
$$

в силу неравенства Бернштейна. Принимая во внимание очевидное равенство $\left\|f_{0}\right\|_{\operatorname{Lip}(\mathbb{T})}=0$, получаем

$\left\|f_{N}\right\|_{\text {Lip }(\mathbb{T})} \leqslant \sum_{n=1}^{N}\left\|f_{n}-f_{n-1}\right\|_{\operatorname{Lip}(\mathbb{T})} \leqslant 3 \sum_{n=1}^{N} 2^{(1-\alpha) n} \leqslant \frac{3}{1-2^{\alpha-1}} 2^{(1-\alpha) N}, \quad N \in \mathbb{Z}_{+}$. 
Пусть $\zeta, \tau \in \mathbb{T}$. Выберем $N \in \mathbb{Z}_{+}$так, чтобы $2^{-N}<|\zeta-\tau| \leqslant 2^{1-N}$. Тогда

$$
\begin{aligned}
|f(\zeta)-f(\tau)| & \leqslant\left|f(\zeta)-f_{N}(\zeta)\right|+\left|f_{N}(\zeta)-f_{N}(\tau)\right|+\left|f_{N}(\xi)-f(\tau)\right| \\
& \leqslant 2\left\|f-f_{N}\right\|_{L^{\infty}}+\left\|f_{N}\right\|_{\text {Lip }}|\zeta-\tau| \\
& \leqslant 2 \cdot 2^{-\alpha N}+\frac{3}{1-2^{\alpha-1}} 2^{(1-\alpha) N}|\zeta-\tau| \\
& \leqslant 2|\zeta-\tau|^{\alpha}+\frac{3 \cdot 2^{1-\alpha}}{1-2^{\alpha-1}}|\zeta-\tau|^{\alpha} \leqslant \frac{8}{1-2^{\alpha-1}}|\zeta-\tau|^{\alpha} .
\end{aligned}
$$

Следующая теорема говорит о том, что всякая функция $f$ из $\Lambda_{\alpha}(\mathbb{T}), 0<$ $\alpha<1$, является операторно гёльдеровой порядка $\alpha$, что резко контрастирует со случаем липшицевых функций.

TeOpема 1.7.1. Пусть $f \in \Lambda_{\alpha}(\mathbb{T})$, где $\alpha \in(0,1)$. Тогда существует константа с такая, что

$$
\|f(U)-f(V)\| \leqslant c(1-\alpha)^{-1}\|f\|_{\Lambda_{\alpha}}\|U-V\|^{\alpha}
$$

для любых унитарных операторов $U u V$.

ДокАзАтельство. Пусть $f \in \Lambda_{\alpha}(\mathbb{T})$. Сначала применяем прямую теорему теории приближений (в данном случае теорему Джексона). В силу этой теоремы

$$
\operatorname{dist}\left(f, \mathcal{P}_{n}\right) \leqslant \operatorname{const}(n+1)^{-\alpha}\|f\|_{\Lambda_{\alpha}}, \quad n \in \mathbb{Z}_{+} .
$$

Повторяя почти слово в слово доказательство соответствующей обратной теоремы, заменяя $\zeta$ и $\tau$ унитарными операторами $U$ и $V$ и применяя операторное неравенство Бернштейна вместо скалярного, получаем искомый результат.

Аналогичное утверждение верно и в случае прямой.

Теорема 1.7.2. Пусть $f \in \Lambda_{\alpha}(\mathbb{R}), \alpha \in(0,1)$. Тогда существует константа с такая, что для любых самосопряжённых операторов $A$ и $B$

$$
\|f(A)-f(B)\| \leqslant c(1-\alpha)^{-1}\|f\|_{\Lambda_{\alpha}}\|A-B\|^{\alpha} .
$$

Доказательство основано на аналогичном описании функций класса $\Lambda_{\alpha}(\mathbb{R})$ в терминах приближения целыми функциями экспоненциального типа.

ПРЯМАЯ ТЕОРЕМА ДЛЯ ПРОСТРАНСТВА $\Lambda_{\alpha}(\mathbb{R})$. Пусть $f \in \Lambda_{\alpha}(\mathbb{R}), 0<\alpha<1$. Тогда существует положительное число с такое, что при всех $\sigma>0$

$$
\inf \left\{h \in \mathscr{E}_{\sigma}:\|f-h\|_{L^{\infty}(\mathbb{R})}\right\} \leqslant c \sigma^{-\alpha}\|f\|_{\Lambda_{\alpha}(\mathbb{T})} .
$$

ОБРАТНАЯ ТЕОРЕМА ДЛЯ ПРОСТРАНСТВА $\Lambda_{\alpha}(\mathbb{R})$. Пустъ $0<\alpha<1$ ufнепрерывная функиия на $\mathbb{R}$ такая, что $\lim _{|x| \rightarrow \infty} x^{-1} f(x)=0$. Предположим, что имеет место (1.7.2) для некоторого положительного числа с и для всех $\sigma>0$. Тогда $f \in \Lambda_{\alpha}(\mathbb{R}) u\|f\|_{\Lambda_{\alpha}(\mathbb{R})} \leqslant 5 c /\left(1-2^{\alpha-1}\right)$.

Более подробно доказательства теорем 1.7 .1 и 1.7.2 изложены в статье [8]. В этой же работе получен и ряд других результатов, основанных, в конечном итоге, на некоторых результатах теории приближений. 
В частности, аналоги теорем 1.7.1 и 1.7.2 получены там для всех $\alpha>0$. Приведём ещё некоторые результаты, полученные в [8], которые также могут быть доказаны методами теории приближений.

Функцию $\omega:[0,+\infty) \rightarrow \mathbb{R}$ будем называть модулем непрерывности, если она является неотрицательной неубывающей непрерывной функцией такой, что $\omega(0)=0, \omega(x)>0$ при $x>0$ и $\omega(x+y) \leqslant \omega(x)+\omega(y)$ для всех $x, y \in[0,+\infty)$.

Обозначим через $\Lambda_{\omega}(\mathbb{R})$ пространство всех непрерывных функций на $\mathbb{R}$ таких, что

$$
\|f\|_{\Lambda_{\omega}(\mathbb{R})} \stackrel{\text { def }}{=} \sup _{x \neq y} \frac{|f(x)-f(y)|}{\omega(|x-y|)}<+\infty .
$$

Аналогичным образом можно определить пространство $\Lambda_{\omega}(\mathbb{T})$.

Положим

$$
\omega_{*}(x) \stackrel{\text { def }}{=} x \int_{x}^{\infty} \frac{\omega(t)}{t^{2}} d t .
$$

Tеорема 1.7.3. Пусть $f \in \Lambda_{\omega}(\mathbb{R})$, где $\omega-$ модуль непреръьности. Тогда

$$
\|f(A)-f(B)\| \leqslant c\|f\|_{\Lambda_{\omega}(\mathbb{R})} \omega_{*}(\|A-B\|)
$$

для любых самосопряљённых операторов $A$ и $B$, где с - абсолютная постоянная.

Аналогичный результат имеет место и для функций $f \in \Lambda_{\omega}(\mathbb{T})$.

\section{8. Гёльдеровские функции при возмущениях операторами класса Шаттена-фон Неймана}

Мы рассмотрим в этом разделе ещё одно приложение операторных неравенств Бернштейна, приведённых в разделе 1.4. Пусть $f$ - функция класса Гёльдера $\Lambda_{\alpha}(\mathbb{R}), 0<\alpha<1$, и пусть $p \geqslant 1$. Предположим, что $A$ и $B$ - самосопряжённые операторы и $B-A \in \boldsymbol{S}_{p}$. Что мы можем сказать об операторе $f(A)-f(B)$ ? Этот вопрос подробно изучался в работе [9]. Здесь мы сформулируем результат работы [9] в случае $p>1$.

TeOpema 1.8.1. Пусть $p>1 u 0<\alpha<1$. Тогдa

$$
\|f(A)-f(B)\|_{S_{p / \alpha}} \leqslant \text { const }\|f\|_{\Lambda_{\alpha}}\|A-B\|_{S_{p}}^{\alpha}
$$

для произвольных самосопряжё̈нных операторов $A$ u $B$, разность которых входит в класс $\boldsymbol{S}_{p}$.

Мы опускаем доказательство теоремы 1.8.1 и отсылаем читателя за доказательством к работе [9]. Случай $p=1$ также подробно рассмотрен в [9]. Заметим, что при $p=1$ заключение теоремы 1.8.1 неверно. В работе [9] также получен аналог теоремы 1.8.1 при всех положительных $\alpha$ и, кроме того, рассматриваются более общие вопросы возмущений операторами из симметрично нормируемых идеалов. 


\section{Глава II. Мультипликаторы Шура и двойные операторные интегралы}

В этой главе мы будем изучать мультипликаторы Шура, как дискретные, так и мультипликаторы Шура по отношению к спектральным мерам. Мы используем описание дискретных мультипликаторов Шура, основанное на теореме Гротендика (см. книгу Ж. Пизье [65] и его работу [66]). Этот результат уточняется в случае, когда исходная функция задана на произведении топологических пространств и является непрерывной по каждой переменной. Получено также уточнение этого результата для борелевских функций, заданных на произведении топологических пространств.

Затем мы определяем двойные операторные интегралы и мультипликаторы Шура по отношению к спектральным мерам. Изучение таких мультипликаторов Шура в случае борелевских функций на произведении топологических пространств сводится к дискретным мультипликаторам Шура.

\section{1. Дискретные мультипликаторы Шура}

Обозначим через $\ell^{p}(\mathcal{T})$ пространство комплексных функций $\alpha: t \mapsto \alpha_{t}$, заданных на не обязательно счётном или конечном множестве $\mathcal{T}$ и таких, что $\sum_{t \in \mathcal{T}}\left|\alpha_{t}\right|^{p}<\infty$, с нормой $\|\alpha\|_{p}=\left(\sum_{t \in \mathcal{T}}\left|\alpha_{t}\right|^{p}\right)^{1 / p}$, где $p \in[1,+\infty)$. При $p=\infty$ пространство $\ell^{p}(\mathcal{T})$ состоит из всех ограниченных комплексных функций $\alpha: t \mapsto$ $\alpha_{t}$, заданных на $\mathcal{T}$, и $\|\alpha\|_{\infty}=\sup _{t \in \mathcal{T}}\left|\alpha_{t}\right|$. В тех случаях, когда необходимо явно указать множество $\mathcal{T}$, на котором задано семейство $\alpha$, мы будем писать $\|\alpha\|_{\ell^{p}(\mathcal{T})}$ вместо $\|\alpha\|_{p}$. Обозначим через $c_{0}(\mathcal{T})$ подпространство пространства $\ell^{\infty}(\mathcal{T})$, состоящее из функций $\alpha$, стремящихся к нулю в бесконечности.

Пусть $\mathcal{S}$ и $\mathcal{T}$ - произвольные непустые множества. Каждому ограниченному оператору $A$, действующему из $\ell^{2}(\mathcal{T})$ в $\ell^{2}(\mathcal{S})$, соответствует единственная

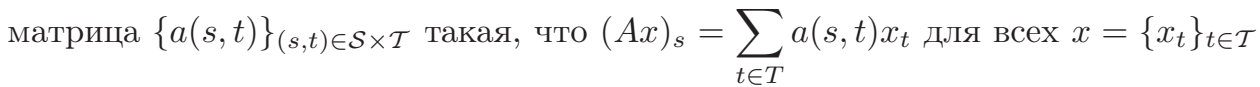
из $\ell^{2}(\mathcal{T})$. В этом случае мы говорим, что матриц $\{a(s, t)\}_{(s, t) \in \mathcal{S} \times \mathcal{T}}$ oпределяет ограниченный оператор $A: \ell^{2}(\mathcal{T}) \rightarrow \ell^{2}(\mathcal{S})$. Положим

$$
\left\|\{a(s, t)\}_{(s, t) \in \mathcal{S} \times \mathcal{T}}\right\| \stackrel{\text { def }}{=}\|A\| \quad \text { и } \quad\left\|\{a(s, t)\}_{(s, t) \in \mathcal{S} \times \mathcal{T}}\right\| \boldsymbol{S}_{1} \stackrel{\text { def }}{=}\|A\|_{\boldsymbol{S}_{1}} .
$$

В случае, когда $A \notin \boldsymbol{S}_{1}\left(\ell^{2}(\mathcal{T}), \ell^{2}(\mathcal{S})\right)$, считаем, что последняя норма равна $\infty$. Если матрица $\{a(s, t)\}(s, t) \in \mathcal{S} \times \mathcal{T}$ не определяет ограниченный оператор из $\ell^{2}(\mathcal{T})$ в $\ell^{2}(\mathcal{S})$, считаем, что её операторная норма (равно как и ядерная) рав-

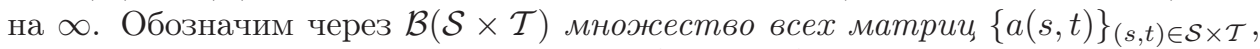
задающих ограниченный оператор из $\ell^{2}(\mathcal{T})$ в $\ell^{2}(\mathcal{S})$. В некоторых случаях

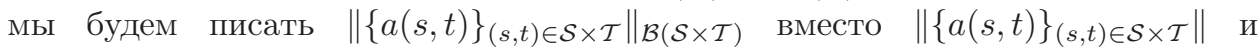
$\left\|\{a(s, t)\}_{(s, t) \in \mathcal{S} \times \mathcal{T}}\right\|_{\boldsymbol{S}_{1}(\mathcal{S} \times \mathcal{T})}$ вместо $\left\|\{a(s, t)\}_{(s, t) \in \mathcal{S} \times \mathcal{T}}\right\|_{\boldsymbol{S}_{1}}$.

Матрица $\Phi=\{\Phi(s, t)\}_{(s, t) \in \mathcal{S} \times \mathcal{T}}$ называется мультипликатором Шура пространства $\mathcal{B}(\mathcal{S} \times \mathcal{T})$, если для любой матрицы $A=\{a(s, t)\}_{(s, t) \in \mathcal{S} \times \mathcal{T}}$ из $\mathcal{B}(\mathcal{S} \times$ $\mathcal{T})$ матрица $\Phi \star A \stackrel{\text { def }}{=}\{\Phi(s, t) a(s, t)\}(s, t) \in \mathcal{S} \times \mathcal{T}$ также принадлежит пространству $\mathcal{B}(\mathcal{S} \times \mathcal{T})$. 
Множество всех мультипликаторов Шура пространства $\mathcal{B}(\mathcal{S} \times \mathcal{T})$ обозначим символом $\mathfrak{M}(\mathcal{S} \times \mathcal{T})$. Из теоремы о замкнутом графике легко вывести, что мультипликаторы Шура порождают ограниченные операторы в $\mathcal{B}(\mathcal{S} \times \mathcal{T})$. Положим

$$
\|\Phi\|_{\mathfrak{M}(\mathcal{S} \times \mathcal{T})} \stackrel{\text { def }}{=} \sup \left\{\|\Phi \star A\|: A \in \mathcal{B}(\mathcal{S} \times \mathcal{T}),\|A\|_{\mathcal{B}} \leqslant 1\right\} .
$$

Отсюда по двойственности получаем, что

$$
\|\Phi\|_{\mathfrak{M}(\mathcal{S} \times \mathcal{T})}=\sup \left\{\|\Phi \star A\|_{\boldsymbol{S}_{1}}: A \in \mathcal{B}(\mathcal{S} \times \mathcal{T}),\|A\|_{S_{1}} \leqslant 1\right\} .
$$

Легко видеть, что

$$
\begin{gathered}
\|A\|_{\mathcal{B}(\mathcal{S} \times \mathcal{T})}=\sup \|A\|_{\mathcal{B}\left(\mathcal{S}_{0} \times \mathcal{T}_{0}\right)}, \quad\|A\|_{\boldsymbol{S}_{1}(S \times \mathcal{T})}=\sup \|A\|_{S_{1}\left(\mathcal{S}_{0} \times \mathcal{T}_{0}\right)}, \\
\|\Phi\|_{\mathfrak{M}(\mathcal{S} \times \mathcal{T})}=\sup \|\boldsymbol{\varphi}\|_{\mathfrak{M}\left(\mathcal{S}_{0} \times \mathcal{T}_{0}\right)},
\end{gathered}
$$

где супремумы берутся по всем конечным подмножествам $\mathcal{S}_{0}$ и $\mathcal{T}_{0}$ множеств $\mathcal{S}$ и $\mathcal{T}$.

Отметим ещё, что $\|\Phi\|_{\ell^{\infty}(\mathcal{S} \times \mathcal{T})} \leqslant\|\Phi\|_{\mathfrak{M}(\mathcal{S} \times \mathcal{T})}$. Легко видеть, что неравенство

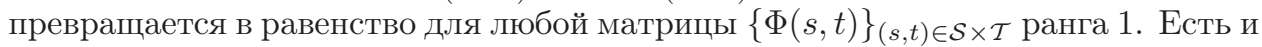
другие классы матриц, для которых это неравенство превращается в равенство. Например, если каждая строка (или каждый столбец) матрицы $\Phi$ содержит не более одного ненулевого элемента, то $\|\Phi\|_{\ell^{\infty}(\mathcal{S} \times \mathcal{T})}=\|\Phi\|_{\mathfrak{M}(\mathcal{S} \times \mathcal{T})}$.

Нам понадобится ещё одна характеристика матрицы $\Phi$. Положим $\|\Phi\|_{\mathfrak{M}_{0}(\mathcal{S} \times \mathcal{T})} \stackrel{\text { def }}{=} \sup \{\|\Phi \star A\|: A \in \mathcal{B}(\mathcal{S} \times \mathcal{T}),\|A\| \leqslant 1, a(t, t)=0$ при $t \in \mathcal{S} \cap \mathcal{T}\}$. Обозначим символом $\mathfrak{M}_{0}(\mathcal{S} \times \mathcal{T})$ множество всех матриц $\Phi=\{\Phi(s, t)\}_{(s, t) \in \mathcal{S} \times \mathcal{T}}$ таких, что $\|\Phi\|_{\mathfrak{M}_{0}(\mathcal{S} \times \mathcal{T})}<+\infty$. Очевидно, что $\|\Phi\|_{\mathfrak{M}_{0}(\mathcal{S} \times \mathcal{T})} \leqslant\|\Phi\|_{\mathfrak{M}(\mathcal{S} \times \mathcal{T})}$. Легко видеть, что

$$
\|\Phi\|_{\mathfrak{M}_{0}(\mathcal{S} \times \mathcal{T})}=\sup \|\Phi\|_{\mathfrak{M}_{0}\left(\mathcal{S}_{0} \times \mathcal{T}_{0}\right)}
$$

где супремум берётся по всем конечным подмножествам $\mathcal{S}_{0}$ и $\mathcal{T}_{0}$ множеств $\mathcal{S}$ и $\mathcal{T}$.

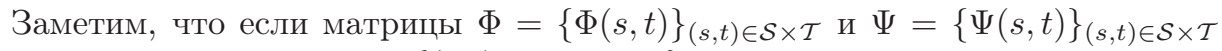
совпадают вне "диагонали" $\{(t, t): t \in \mathcal{S} \cap \mathcal{T}\}$, то

$$
\|\Phi-\Psi\|_{\mathfrak{M}_{0}(\mathcal{S} \times \mathcal{T})}=0 \quad \text { и } \quad\|\Phi\|_{\mathfrak{M}_{0}(\mathcal{S} \times \mathcal{T})}=\|\Psi\|_{\mathfrak{M}_{0}(\mathcal{S} \times \mathcal{T})} .
$$

Отметим, что $\|\Phi\|_{\mathfrak{M}_{0}(\mathcal{S} \times \mathcal{T})}=\|\Phi\|_{\mathfrak{M}(\mathcal{S} \times \mathcal{T})}$, если $\mathcal{S} \cap \mathcal{T}=\varnothing$.

Лемма 2.1.1. Пусть $\Phi \in \ell^{\infty}(\mathcal{S} \times \mathcal{T})$, где $\mathcal{S} u \mathcal{T}$ - произвольные множества mакие, что $\mathcal{S} \cap \mathcal{T} \neq \varnothing$. Тогда

$$
\begin{aligned}
\max \left\{\|\Phi\|_{\mathfrak{M}_{0}(\mathcal{S} \times \mathcal{T})},\|\Phi(t, t)\|_{\ell^{\infty}(\mathcal{S} \cap \mathcal{T})}\right\} & \leqslant\|\Phi\|_{\mathfrak{M}(\mathcal{S} \times \mathcal{T})} \\
& \leqslant 2\|\Phi\|_{\mathfrak{M}_{0}(\mathcal{S} \times \mathcal{T})}+\|\Phi(t, t)\|_{\ell^{\infty}(\mathcal{S} \cap \mathcal{T})} .
\end{aligned}
$$

ДокАзАтельство. Первое неравенство очевидно. Докажем второе. Обозначим символом $\chi$ характеристическую функцию множества $\{(s, t) \in \mathcal{S} \times \mathcal{T}: s=t\}$. Легко видеть, что $\|\chi\|_{\mathfrak{M}(\mathcal{S} \times \mathcal{T})}=1$, откуда $\|1-\chi\|_{\mathfrak{M}(\mathcal{S} \times \mathcal{T})} \leqslant\|1\|_{\mathfrak{M}(\mathcal{S} \times \mathcal{T})}+$ $\|\chi\|_{\mathfrak{M}(\mathcal{S} \times \mathcal{T})}=2$. Пусть $A \in \mathcal{B}(\mathcal{S} \times \mathcal{T})$ и $\|A\| \leqslant 1$. Тогда $\Phi \star A=\Phi \star(1-\chi) \star A+$ $\Phi \star \chi \star A$. Остаётся заметить, что

$$
\begin{gathered}
\|\Phi \star(1-\chi) \star A\| \leqslant\|\Phi\|_{\mathfrak{M}_{0}(\mathcal{S} \times \mathcal{T})}\|(1-\chi) \star A\| \leqslant 2\|\Phi\|_{\mathfrak{M}_{0}(\mathcal{S} \times \mathcal{T})}, \\
\|\Phi \star \chi \star A\| \leqslant\|\Phi \star \chi\|_{\mathfrak{M}(\mathcal{S} \times \mathcal{T})}=\|\Phi(t, t)\|_{\ell^{\infty}(\mathcal{S} \cap \mathcal{T}) .}
\end{gathered}
$$

Лемма доказана. 
СлЕДСТвиЕ 2.1.2. Если $\Phi(t, t)=0$ при всех $t \in \mathcal{S} \cap \mathcal{T}, m o$

$$
\|\Phi\|_{\mathfrak{M}_{0}(\mathcal{S} \times \mathcal{T})} \leqslant\|\Phi\|_{\mathfrak{M}(\mathcal{S} \times \mathcal{T})} \leqslant 2\|\Phi\|_{\mathfrak{M}_{0}(\mathcal{S} \times \mathcal{T})} .
$$

Лемма 2.1.3. Пусть $\mathcal{S}$ - хаусдорфово топологические пространство. Предположим, что никакая точка множества $\mathcal{S} \cap \mathcal{T}$ не является изолированной точкой в пространстве $\mathcal{S}$. Тогда $\|\Phi\|_{\mathfrak{M}_{0}(\mathcal{S} \times \mathcal{T})}=\|\Phi\|_{\mathfrak{M}(\mathcal{S} \times \mathcal{T})}$ для любой функици $\Phi \in \ell^{\infty}(\mathcal{S} \times \mathcal{T})$, непрерывной по переменной $s \in \mathcal{S}$.

ДоказАтельство. Достаточно доказать, что $\|\Phi\|_{\mathfrak{M}(\mathcal{S} \times \mathcal{T})} \leqslant\|\Phi\|_{\mathfrak{M}_{0}(\mathcal{S} \times \mathcal{T})}$ или, что то же самое, $\|\Phi\|_{\mathfrak{M}\left(\mathcal{S}_{0} \times \mathcal{T}_{0}\right)} \leqslant\|\Phi\|_{\mathfrak{M}_{0}(\mathcal{S} \times \mathcal{T})}$ для всех конечных подмножеств $\mathcal{S}_{0}$ и $\mathcal{T}_{0}$ множеств $\mathcal{S}$ и $\mathcal{T}$. Зафиксируем конечные подмножества $\mathcal{S}_{0}$ и $\mathcal{T}_{0}$ множеств $\mathcal{S}$ и $\mathcal{T}$. Ясно, что для любого положительного числа $\varepsilon$ существует возмущение $\widetilde{\mathcal{S}}_{0}$ множества $\mathcal{S}_{0}$ такое, что $\widetilde{\mathcal{S}}_{0} \cap \mathcal{T}_{0}=\varnothing$ и $\|\Phi\|_{\mathfrak{M}\left(\mathcal{S}_{0} \times \mathcal{T}_{0}\right)<}$ $\varepsilon+\|\Phi\|_{\mathfrak{M}\left(\widetilde{\mathcal{S}}_{0} \times \mathcal{T}_{0}\right)} \cdot$ Следовательно,

$$
\|\Phi\|_{\mathfrak{M}\left(\mathcal{S}_{0} \times \mathcal{T}_{0}\right)}<\varepsilon+\|\Phi\|_{\mathfrak{M}\left(\widetilde{\mathcal{S}}_{0} \times \mathcal{T}_{0}\right)}=\varepsilon+\|\Phi\|_{\mathfrak{M}_{0}\left(\widetilde{\mathcal{S}}_{0} \times \mathcal{T}_{0}\right)} \leqslant \varepsilon+\|\Phi\|_{\mathfrak{M}_{0}(\mathcal{S} \times \mathcal{T})}
$$

для любого $\varepsilon>0$. Лемма доказана.

Мы собираемся рассмотреть аналог пространства $\mathfrak{M}_{0}(\mathcal{S} \times \mathcal{T})$, определённый через $\boldsymbol{S}_{1}$-норму вместо операторной нормы. Для этого мы положим

$$
\begin{array}{r}
\|\Phi\|_{\mathfrak{M}_{0, S_{1}}(\mathcal{S} \times \mathcal{T})} \stackrel{\text { def }}{=} \sup \left\{\|\Phi \star A\|_{\boldsymbol{S}_{1}}: A \in \mathcal{B}(\mathcal{S} \times \mathcal{T}),\|A\|_{\boldsymbol{S}_{1}} \leqslant 1,\right. \\
a(t, t)=0 \text { для } t \in \mathcal{S} \cap \mathcal{T}\}
\end{array}
$$

и $\mathfrak{M}_{0, S_{1}}(\mathcal{S} \times \mathcal{T}) \stackrel{\text { def }}{=}\left\{\Phi:\|\Phi\|_{\mathfrak{M}_{0, S_{1}}(\mathcal{S} \times \mathcal{T})}<+\infty\right\}$. При этом следует отметить, что соответствующий аналог пространства $\mathfrak{M}(\mathcal{S} \times \mathcal{T})$ нам не нужно определять, ибо он совпадает с этим же пространством $\mathfrak{M}(\mathcal{S} \times \mathcal{T})$ в силу равенства (2.1.1).

Точно так же, как для операторной нормы, можно доказать следующие утверждения.

ЛЕмма 2.1.4. Пусть $\Phi \in \ell^{\infty}(\mathcal{S} \times \mathcal{T})$, где $\mathcal{S} u \mathcal{T}$ - произвольные множества mакие, ито $\mathcal{S} \cap \mathcal{T} \neq \varnothing$. Тогда

$$
\begin{aligned}
\max \left\{\|\Phi\|_{\mathfrak{M}_{0, S_{1}}(\mathcal{S} \times \mathcal{T})},\|\Phi(t, t)\|_{\ell^{\infty}(\mathcal{S} \cap \mathcal{T})}\right\} & \leqslant\|\Phi\|_{\mathfrak{M}(\mathcal{S} \times \mathcal{T})} \\
& \leqslant 2\|\Phi\|_{\mathfrak{M}_{0, S_{1}}(\mathcal{S} \times \mathcal{T})}+\|\Phi(t, t)\|_{\ell^{\infty}(\mathcal{S} \cap \mathcal{T})} .
\end{aligned}
$$

Если $\Phi(t, t)=0$ при всех $t \in \mathcal{S} \cap \mathcal{T}$, то

$$
\|\Phi\|_{\mathfrak{M}_{0, S_{1}}(\mathcal{S} \times \mathcal{T})} \leqslant\|\Phi\|_{\mathfrak{M}(\mathcal{S} \times \mathcal{T})} \leqslant 2\|\Phi\|_{\mathfrak{M}_{0, S_{1}}(\mathcal{S} \times \mathcal{T})} .
$$

СлеДСтвие 2.1.5. Если $\Phi(t, t)=0$ при всех $t \in \mathcal{S} \cap \mathcal{T}, m o$

$$
\|\Phi\|_{\mathfrak{M}_{0, S_{1}}(\mathcal{S} \times \mathcal{T})} \leqslant\|\Phi\|_{\mathfrak{M}(\mathcal{S} \times \mathcal{T})} \leqslant 2\|\Phi\|_{\mathfrak{M}_{0, S_{1}}(\mathcal{S} \times \mathcal{T})} .
$$

Лемма 2.1.6. Пусть $\mathcal{S}$ - хаусдорфово топологические пространство. Предположим, что никакая точка множества $\mathcal{S} \cap \mathcal{T}$ не является изолированной точкой в пространстве $\mathcal{S}$. Тогда $\|\Phi\|_{\mathfrak{M}_{0, \mathcal{S}_{1}}(\mathcal{S} \times \mathcal{T})}=\|\Phi\|_{\mathfrak{M}(\mathcal{S} \times \mathcal{T})}$ для любой функиии $\Phi$ из $\ell^{\infty}(\mathcal{S} \times \mathcal{T})$, непрерывной по переменной $s \in \mathcal{S}$. 


\section{2. Описание дискретных мультипликаторов Шура}

ТеОрема 2.2.1. Пусть $\left\{u_{s}\right\}_{s \in \mathcal{S}} u\left\{v_{t}\right\}_{t \in \mathcal{T}}$ - семейства векторов в (не обязательно сепарабельном) гильбертовом пространстве $\mathscr{H}$ такие, что $\left\|u_{s}\right\| \times$ $\left\|v_{t}\right\| \leqslant 1$ для всех $s$ из $\mathcal{S}$ и $t$ из $\mathcal{T}$. Положим $\Phi(s, t) \stackrel{\text { def }}{=}\left(u_{s}, v_{t}\right), s \in \mathcal{S}, t \in \mathcal{T}$. Тогда $\Phi \in \mathfrak{M}(\mathcal{S} \times \mathcal{T}) u\|\Phi\|_{\mathfrak{M}(\mathcal{S} \times \mathcal{T})} \leqslant 1$.

ДокАзАТЕльство. В силу (2.1.1) достаточно доказать, что

$$
\left\|\left\{a(s, t)\left(u_{s}, v_{t}\right)\right\}\right\|_{S_{1}} \leqslant\|\{a(s, t)\}\|_{S_{1}}
$$

для любой матрицы $\{a(s, t)\}$, задающей ядерный оператор. Ясно, что достаточно ограничиться случаем, когда $\operatorname{rank}\{a(s, t)\}=1$. Кроме того, можно считать, что $\|a(s, t)\|_{S_{1}}=1$. Тогда $a(s, t)=\alpha_{s} \beta_{t}$ для некоторых $\alpha \in \ell^{2}(\mathcal{S})$ и $\beta \in \ell^{2}(\mathcal{T})$ таких, что $\|\alpha\|_{\ell^{2}(\mathcal{S})}=\|\beta\|_{\ell^{2}(\mathcal{T})}=1$. Пусть $\left\{e_{j}\right\}_{j \in J}-$ ортонормированный базис в гильбертовом пространстве $\mathscr{H}$. Положим $\widehat{x}(j) \stackrel{\text { def }}{=}\left(x, e_{j}\right), j \in J$. Тогда

$$
\begin{aligned}
\left\|\left\{\alpha_{s} \beta_{t}\left(u_{s}, v_{t}\right)\right\}\right\|_{\boldsymbol{S}_{1}} & \left.\leqslant \sum_{j \in J} \|\left\{\alpha_{s} \beta_{t} \widehat{u}_{s}(j) \overline{\widehat{v}_{t}(j)}\right)\right\} \|_{\boldsymbol{S}_{1}} \\
& =\sum_{j \in J}\left\|\left\{\alpha_{s} \widehat{u}_{s}(j)\right\}\right\|_{\ell^{2}(\mathcal{S})}\left\|\left\{\beta_{t} \overline{\widehat{v}_{t}(j)}\right\}\right\|_{\ell^{2}(\mathcal{T})} \\
& \leqslant\left(\sum_{j \in J}\left\|\left\{\alpha_{s} \widehat{u}_{s}(j)\right\}\right\|_{\ell^{2}(\mathcal{S})}^{2}\right)^{1 / 2}\left(\sum_{j \in J}\left\|\left\{\beta_{t} \overline{\widehat{v}_{t}(j)}\right\}\right\|_{\ell^{2}(\mathcal{T})}^{2}\right)^{1 / 2} .
\end{aligned}
$$

Ясно, что

$$
\begin{aligned}
\sum_{j \in J}\left\|\left\{\alpha_{s} \widehat{u}_{s}(j)\right\}\right\|_{\ell^{2}(\mathcal{S})}^{2} & =\sum_{j \in J} \sum_{s \in \mathcal{S}}\left|\alpha_{s}\right|^{2}\left|\widehat{u}_{s}(j)\right|^{2} \\
& =\sum_{s \in \mathcal{S}}\left|\alpha_{s}\right|^{2} \sum_{j \in J}\left|\widehat{u}_{s}(j)\right|^{2}=\sum_{s \in \mathcal{S}}\left|\alpha_{s}\right|^{2}\left\|u_{s}\right\|^{2} \leqslant \sup _{s \in \mathcal{S}}\left\|u_{s}\right\|^{2} .
\end{aligned}
$$

Аналогичным образом, $\sum_{j \in J}\left\|\left\{\beta_{t} \overline{\widehat{v}_{t}(j)}\right\}\right\|_{\ell^{2}(\mathcal{T})}^{2} \leqslant \sup _{t \in \mathcal{T}}\left\|v_{t}\right\|^{2}$. Отсюда следует, что

$$
\left\|\left\{\alpha_{s} \beta_{t}\left(u_{s}, v_{t}\right)\right\}\right\| S_{1} \leqslant \sup _{s \in \mathcal{S}}\left\|u_{s}\right\| \sup _{t \in \mathcal{T}}\left\|u_{t}\right\| \leqslant 1
$$

Теорема доказана.

Весьма нетривиальным является обратное утверждение, которое содержится в теореме 5.1 монографии [65] (см. также [66]). Этот результат мы приводим без доказательства.

TЕорема 2.2.2. Пусть $\Phi \stackrel{\text { def }}{=}\{\Phi(s, t)\}$ - мультипликатор Шура на пространстве $\mathcal{B}(\mathcal{S} \times \mathcal{T})$ u $\|\Phi\|_{\mathfrak{M}} \leqslant 1$. Тогда найдутся два семейства векторов $\left\{u_{s}\right\}_{s \in \mathcal{S}} u\left\{v_{t}\right\}_{t \in \mathcal{T}}$ в (не обязательно сепарабельном) гилъбертовом пространстве $\mathscr{H}$ такие, что $\left\|u_{s}\right\| \leqslant 1$ при всех $s \in \mathcal{S},\left\|v_{t}\right\| \leqslant 1$ при всех $t \in \mathcal{T} u$

$$
\Phi(s, t)=\left(u_{s}, v_{t}\right), \quad s \in \mathcal{S}, \quad t \in \mathcal{T} .
$$


ЗАМЕЧАНИЕ К ТЕОРЕМЕ 2.2.2. В этой теореме можно дополнителъно потребовать, чтобы линейная оболочка как семейства $\left\{u_{s}\right\}_{s \in \mathcal{S}}$, так и семейcтва $\left\{v_{t}\right\}_{t \in \mathcal{T}}$ была всюду плотна в гилъбертовом пространстве $\mathscr{H}$. Действительно, пусть $\mathscr{H}_{1}$ - замыкание линейной оболочки семейства $\left\{v_{t}\right\}_{t \in \mathcal{T}}$ и пусть $P_{1}$ - ортогональный проектор на $\mathscr{H}_{1}$. Тогда $\left\{P_{1} u_{s}\right\}_{s \in \mathcal{S}}$ и $\left.v_{t}\right\}_{t \in \mathcal{T}}$ - семейства в гильбертовом пространстве $\mathscr{H}_{1}$ такие, что $\Phi(s, t)=\left(P_{1} u_{s}, v_{t}\right)$ для всех $(s, t) \in$ $\mathcal{S} \times \mathcal{T}$. Пусть теперь $\mathscr{H}_{2}$ - замыкание линейной оболочки семейства $\left\{P_{1} u_{s}\right\}_{s \in \mathcal{S}}$, a $P_{2}$ - ортогональный проектор на $\mathscr{H}_{2}$. Тогда $\left\{P_{1} u_{s}\right\}_{s \in \mathcal{S}}$ и $\left\{P_{2} v_{t}\right\}_{t \in \mathcal{T}}-$ семейства векторов в $\mathscr{H}_{2}$ такие, что $\Phi(s, t)=\left(P_{1} u_{s}, P_{2} v_{t}\right)$ при $(s, t) \in \mathcal{S} \times \mathcal{T}$. Ясно, что линейные оболочки семейств $\left\{P_{1} u_{s}\right\}_{s \in \mathcal{S}}$ и $\left\{P_{2} v_{t}\right\}_{t \in \mathcal{T}}$ плотны в гильбертовом пространстве $\mathscr{H}_{2}$.

Следующая теорема содержится в результатах работ [39] и [5].

Tеорема 2.2.3. Пусть $\Phi \in \mathfrak{M}(\mathcal{S} \times \mathcal{T})$, где $\mathcal{S}$ u $\mathcal{T}$ - топологические пространства. Предположим, что функиия $\Phi$ непрерывна по каждой переменной. Тогда существуют два семейства $\left\{u_{s}\right\}_{s \in \mathcal{S}} u\left\{v_{t}\right\}_{t \in \mathcal{T}}$ в (не обязательно сепарабельном) гильбертовом пространстве $\mathscr{H}$ такие, что

(a) линейная оболочка семейства $\left\{u_{s}\right\}_{s \in \mathcal{S}}$ плотна в $\mathscr{H}$;

(b) линейная оболочка семейства $\left\{v_{t}\right\}_{t \in \mathcal{T}}$ плотна в $\mathscr{H}$;

(c) отображение $s \mapsto u_{s}$ слабо непрерьвно;

(d) отображение $t \mapsto v_{t}$ слабо непрерывно;

(e) $\left\|u_{s}\right\|^{2} \leqslant\|\Phi\|_{\mathfrak{M}(\mathcal{S} \times \mathcal{T})}$ для всех $s \in \mathcal{S}$;

(f) $\left\|v_{t}\right\|^{2} \leqslant\|\Phi\|_{\mathfrak{M}(\mathcal{S} \times \mathcal{T})}$ для всех $t \in \mathcal{T}$;

(g) $\Phi(s, t)=\left(u_{s}, v_{t}\right)$ для всех $(s, t) \in \mathcal{S} \times \mathcal{T}$.

ДокАЗАТЕЛЬСтво. В силу теоремы 2.2.2 и замечания к ней найдутся семейства $\left\{u_{s}\right\}_{s \in \mathcal{S}}$ и $\left\{v_{t}\right\}_{t \in \mathcal{T}}$, удовлетворяющие условиям (a), (b), (e), (f) и (g). Ясно, что функция $s \mapsto\left(u_{s}, h\right)$ непрерывна для $h=v_{t}$, где $t \in \mathcal{T}$. Следовательно, функция $s \mapsto\left(u_{s}, h\right)$ непрерывна при всех $h \in \mathscr{H}$ в силу (b). Таким образом, отображение $s \mapsto u_{s}$ слабо непрерывно. Аналогично из (а) выводится слабая непрерывность отображения $t \mapsto v_{t}$. Теорема доказана.

ЗАмЕчаниЕ. Если хотя бы одно из пространств $\mathcal{S}$ и $\mathcal{T}$ сепарабельно, то и пространство $\mathscr{H}$ сепарабельно. Действительно, достаточно заметить, что если, например, $\mathcal{S}$ сепарабельно, то замыкание линейной оболочки семейства $\left\{u_{s}\right\}_{s \in S}$ сепарабельно.

Это замечание позволяет получить следующее утверждение.

Теорема 2.2.4. Пусть $\Phi \in \mathfrak{M}(\mathcal{S} \times \mathcal{T})$, где $\mathcal{S}$ u $\mathcal{T}$ - топологические пространства, причём хотя бъ одно из них сепарабельно. Предположим, что функиия $\Phi$ непрерывна по каждой переменной. Тогда существуют последовательность непрерывных функиий $\left\{\varphi_{n}\right\}_{n \geqslant 1}$ на множестве $\mathcal{S}$ и последовательность непрерывных функиий $\left\{\psi_{n}\right\}_{n \geqslant 1}$ на множестве $\mathcal{T}$ такие, что при всех $s \in \mathcal{S} u t \in \mathcal{T}$

$$
\begin{gathered}
\sum_{n=1}^{\infty}\left|\varphi_{n}(s)\right|^{2} \leqslant\|\Phi\|_{\mathfrak{M}(\mathcal{S} \times \mathcal{T})}, \quad \sum_{n=1}^{\infty}\left|\psi_{n}(t)\right|^{2} \leqslant\|\Phi\|_{\mathfrak{M}(\mathcal{S} \times \mathcal{T})}, \\
\sum_{n=1}^{\infty} \varphi_{n}(s) \psi_{n}(t)=\Phi(s, t) .
\end{gathered}
$$


ДоказАтельство. Пусть $\left\{u_{s}\right\}_{s \in \mathcal{S}}$ и $\left\{v_{t}\right\}_{t \in \mathcal{T}}-$ два семейства в гильбертовом пространстве $\mathscr{H}$, существующих по теореме 2.2.3. Из замечания к этой теореме следует, что пространство $\mathscr{H}$ сепарабельно. Пусть $\left\{e_{n}\right\}_{n=1}^{N}$ - ортонормированный базис в $\mathscr{H}$, где $0 \leqslant N \leqslant \infty$. Остаётся положить $\varphi_{n}(s) \stackrel{\text { def }}{=}\left(u_{s}, e_{n}\right)$ и $\psi_{n}(t) \stackrel{\text { def }}{=}\left(e_{n}, v_{t}\right)$; при этом если $N<\infty$, то $\varphi_{n}(s) \stackrel{\text { def }}{=} \psi_{n}(t) \stackrel{\text { def }}{=} 0$ при $n>N$. Теорема доказана.

ОПРЕДЕЛЕНИЕ. Отображение $g$, действующее из топологического пространства $\mathcal{T}$ в гильбертово пространство $\mathscr{H}$, называется слабо измеримым по Борелю, если функция $t \mapsto(g(t), u)$ измерима по Борелю на $T$ для любого вектора $u$ из $\mathscr{H}$.

Легко видеть, что условие измеримости по Борелю функции $t \mapsto(g(t), u)$ достаточно проверить только для векторов $u$ из подмножества гильбертова пространства $\mathscr{H}$, линейная оболочка которого плотна в $\mathscr{H}$.

Теорема 2.2.5. Пусть $\mathcal{S}$ u $\mathcal{T}$ - топологические пространства, а $\Phi \in$ $\mathfrak{M}(\mathcal{S} \times \mathcal{T})$. Предположим, что $\Phi$ измерима по Борелю по каждой переменной. Тогда существуют два семейства $\left\{u_{s}\right\}_{s \in \mathcal{S}} u\left\{v_{t}\right\}_{t \in \mathcal{T}}$ в (не обязательно сепарабельном) гильбертовом пространстве $\mathscr{H}$ такие, что

(a) линейная оболочка семейства $\left\{u_{s}\right\}_{s \in \mathcal{S}}$ плотна в $\mathscr{H}$;

(b) линейная оболочка семейства $\left\{v_{t}\right\}_{t \in \mathcal{T}}$ плотна в $\mathscr{H}$;

(c) отображение $s \mapsto u_{s}$ измеримо по Борелю в слабом смысле;

(d) отображение $t \mapsto v_{t}$ измеримо по Борелю в слабом смысле;

(e) $\left\|u_{s}\right\|^{2} \leqslant\|\Phi\|_{\mathfrak{M}(\mathcal{S} \times \mathcal{T})}$ для всех $s \in \mathcal{S}$;

(f) $\left\|v_{t}\right\|^{2} \leqslant\|\Phi\|_{\mathfrak{M}(\mathcal{S} \times \mathcal{T})}$ для всех $t \in \mathcal{T}$;

(g) $\Phi(s, t)=\left(u_{s}, v_{t}\right)$ для всех $(s, t) \in \mathcal{S} \times \mathcal{T}$.

Доказательство теоремы 2.2.5 по существу слово в слово повторяет доказательство теоремы 2.2.3.

Теорема 2.2.6. Пусть $\mathcal{S}$ u $\mathcal{T}$ - топологические пространства, а $\Phi-$-борелевская функиия из $\mathfrak{M}(\mathcal{S} \times \mathcal{T})$. Предположим, что $\mu$ и $\nu$ - борелевские $\sigma$-конечные меры на $\mathcal{S} u \mathcal{T}$. Тогда существуют последовательности $\left\{\varphi_{k}\right\}_{k \geqslant 1}$ $u\left\{\psi_{k}\right\}_{k \geqslant 1}$ maкue, чmo

(a) $\varphi_{k} \in L^{\infty}(\mu) u \psi_{k} \in L^{\infty}(\nu)$ nрu всех $k \geqslant 1$;

(b) $\sum_{k=1}^{\infty}\left|\varphi_{k}(s)\right|^{2} \leqslant\|\Phi\|_{\mathfrak{M}(\mathcal{S} \times \mathcal{T})}$ nри $\mu$-почти всех $s \in \mathcal{S}$;

(c) $\sum_{k=1}^{\infty}\left|\psi_{k}(t)\right|_{\infty}^{2} \leqslant\|\Phi\|_{\mathfrak{M}(\mathcal{S} \times \mathcal{T})}$ nри $\nu$-почти всех $t \in \mathcal{T}$;

(d) $\Phi(s, t)=\sum_{k=1}^{\infty} \varphi_{k}(s) \psi_{k}(t)$ при $\mu \otimes \nu$-почти всех $(s, t) \in \mathcal{S} \times \mathcal{T}$.

ДоКАЗАТЕЛЬСтво. Ясно, что можно считать, что $\|\Phi\|_{\mathfrak{M}(\mathcal{S} \times \mathcal{T})}=1$. Пусть $\left\{u_{s}\right\}_{s \in \mathcal{S}}$ и $\left\{v_{t}\right\}_{t \in \mathcal{T}}-$ семейства в гильбертовом пространстве $\mathscr{H}$, существование которых утверждается в теореме 2.2.5. Пусть $\left\{e_{j}\right\}_{j \in J}$ - ортонормированный базис в гильбертовом пространстве $\mathscr{H}$. Положим $\varphi_{j}(s) \stackrel{\text { def }}{=}\left(u_{s}, e_{j}\right)$ 
и $\psi_{j}(t) \stackrel{\text { def }}{=}\left(e_{j}, v_{t}\right)$. Тогда функции $\varphi_{j}$ и $\psi_{j}$ измеримы по Борелю,

$$
\sum_{j \in J}\left|\varphi_{j}(s)\right|^{2} \leqslant 1 \quad \text { при всех } s \in \mathcal{S}, \quad \sum_{j \in J}\left|\psi_{j}(t)\right|^{2} \leqslant 1 \quad \text { при всех } t \in \mathcal{T}
$$

и

$$
\Phi(s, t)=\sum_{j \in J} \varphi_{j}(s) \psi_{j}(t) \quad \text { при всех }(s, t) \in \mathcal{S} \times \mathcal{T} .
$$

Отсюда мгновенно вытекает доказываемая теорема в случае, когда множество $J$ не более чем счётно. Чтобы рассмотреть случай произвольного множества $J$, положим $\Psi(s, t) \stackrel{\text { def }}{=} \sum_{j \in J}\left|\varphi_{j}(s)\right| \cdot\left|\psi_{j}(t)\right|$. По неравенству Коши-Буня-
ковского

$$
\Psi(s, t) \leqslant\left(\sum_{j \in J}\left|\varphi_{j}(s)\right|^{2}\right)^{1 / 2}\left(\sum_{j \in J}\left|\psi_{j}(t)\right|^{2}\right)^{1 / 2} \leqslant 1
$$

Можно считать, что меры $\mu$ и $\nu$ вероятностные. Пусть $J_{s} \stackrel{\text { def }}{=}\left\{j \in J: \varphi_{j}(s) \neq 0\right\}$, где $s \in \mathcal{S}$. Заметим, что множество $J_{s}$ не более чем счётно при каждом $s \in \mathcal{S}$, поскольку $\sum_{j \in J}\left|\varphi_{j}(s)\right|^{2} \leqslant 1$. Легко видеть, что для всех $s$ из $\mathcal{S}$

$$
\begin{aligned}
\sum_{j \in J}\left|\varphi_{j}(s)\right| \int_{\mathcal{T}}\left|\psi_{j}(t)\right| d \nu(t) & =\sum_{j \in J_{s}}\left|\varphi_{j}(s)\right| \int_{\mathcal{T}}\left|\psi_{j}(t)\right| d \nu(t) \\
& =\int_{\mathcal{T}}\left(\sum_{j \in J_{s}}\left|\varphi_{j}(s)\right| \cdot\left|\psi_{j}(t)\right|\right) d \nu(t)=\int_{\mathcal{T}} \Psi(s, t) d \nu(t) .
\end{aligned}
$$

Чтобы проинтегрировать теперь по $s$, введём не более чем счётное множество $J_{b} \stackrel{\text { def }}{=}\left\{j \in J: \int_{T}\left|\psi_{j}(t)\right| d \nu(t) \neq 0\right\}$. Тогда

$$
\begin{aligned}
\sum_{j \in J} \int_{\mathcal{S}}\left|\varphi_{j}(s)\right| d \mu(s) \int_{\mathcal{T}}\left|\psi_{j}(t)\right| d \nu(t) & =\sum_{j \in J_{b}} \int_{\mathcal{S}}\left|\varphi_{j}(s)\right| d \mu(s) \int_{\mathcal{T}}\left|\psi_{j}(t)\right| d \nu(t) \\
& =\int_{\mathcal{S}}\left(\int_{\mathcal{T}} \Psi(s, t) d \nu(t)\right) d \mu(s) .
\end{aligned}
$$

Теперь ясно, что $\int_{\mathcal{S}}\left(\int_{\mathcal{T}} \sum_{j \in J \backslash J_{b}}\left|\varphi_{j}(s)\right| \cdot\left|\psi_{j}(t)\right| d \nu(t)\right) d \mu(s)=0$. Отсюда и из неравенства $\left|\Phi(s, t)-\sum_{j \in J_{b}} \varphi_{j}(s) \psi_{j}(t)\right| \leqslant \sum_{j \in J \backslash J_{b}}\left|\varphi_{j}(s)\right| \cdot\left|\psi_{j}(t)\right|$ вытекает, что

$$
\sum_{j \in J_{b}} \varphi_{j}(s) \psi_{j}(t)=\Phi(s, t)
$$

для $\mu \otimes \nu$-почти всех $(s, t) \in \mathcal{S} \times \mathcal{T}$. Теорема доказана.

Рассмотрим некоторые примеры мультипликаторов Шура. Пусть $\mathcal{M}\left(\mathbb{T}^{2}\right)$ обозначает пространство всех комплексных борелевских мер на двухмерном торе $\mathbb{T}^{2}$ с нормой $\|\mu\|_{\mathcal{M}\left(\mathbb{T}^{2}\right)} \stackrel{\text { def }}{=}|\mu|(\mathbb{T})$.

ПримеР. Пусть $\mu \in \mathcal{M}\left(\mathbb{T}^{2}\right)$. Тогда $\{\widehat{\mu}(m, n)\} \in \mathfrak{M}\left(\mathbb{Z}^{2}\right)$ и $\|\widehat{\mu}\|_{\mathfrak{M}(\mathbb{Z} \times \mathbb{Z})} \leqslant\|\mu\|$. 
Это утверждение является очевидным следствием теоремы 2.2.1. Ясно, что далеко не каждый мультипликатор Шура $a \in \mathfrak{M}(\mathbb{Z} \times \mathbb{Z})$ представим в виде $\boldsymbol{a}=\widehat{\mu}$, где $\mu \in \mathcal{M}\left(\mathbb{T}^{2}\right)$. Рассмотрим, например, случай, когда матрица $\boldsymbol{a}=\left\{a_{m n}\right\}_{m, n \in \mathbb{Z}}$ состоит из одинаковых столбцов (или строк). Пусть для определённости $a_{m n}=t_{n}$ при всех $m, n \in \mathbb{Z}$. Тогда $\boldsymbol{a} \in \mathfrak{M}(\mathbb{Z} \times \mathbb{Z})$ в том и только том случае, когда $\boldsymbol{a} \in \ell^{\infty}(\mathbb{Z} \times \mathbb{Z})$ и $\|\boldsymbol{a}\|_{\mathfrak{M}(\mathbb{Z} \times \mathbb{Z})}=\|\boldsymbol{a}\|_{\ell^{\infty}(\mathbb{Z} \times \mathbb{Z})}=\left\|\left\{t_{n}\right\}\right\|_{\ell^{\infty}}$. Разумеется, отнюдь не каждая такая матрица $\boldsymbol{a}$ с ограниченными коэффициентами представима в виде $\boldsymbol{a}=\widehat{\mu}$, где $\mu \in \mathcal{M}\left(\mathbb{T}^{2}\right)$.

С другой стороны, если предположить, что матрица $\boldsymbol{a}=\left\{a_{m n}\right\}_{m, n \in \mathbb{Z}}$ является матрицей Лорана, т. е. $a_{m n}=t_{m-n}$, то ситуация резко изменится.

Tеорема 2.2.7. Пусть $A=\left\{a_{m n}\right\}_{m, n \in \mathbb{Z}}-$ матрица Лорана. Тогда $A \in$ $\mathfrak{M}\left(\mathbb{Z}^{2}\right)$ в том и только том случае, когда $a_{m n}=\widehat{\mu}(m-n)$ для некоторой меры $\mu$ из $\mathcal{M}(\mathbb{T}) ;$ при этом $\|\boldsymbol{a}\|_{\mathfrak{M}\left(\mathbb{Z}^{2}\right)}=\|\mu\|_{\mathcal{M}(\mathbb{T})}$.

Все эти результаты переносятся на локально компактные абелевы группы. При этом в случае недискретной абелевой группы $G$ в формулировке аналога теоремы 2.2.7 нужно потребовать непрерывность соответствующих функций.

Теорема 2.2.8. Пусть $h$ - непрерьвная функиия на $\mathbb{R}$. Тогда матрица $A=\{h(s-t)\}_{s, t \in \mathbb{R}}$ принадлежит пространству $\mathfrak{M}(\mathbb{R} \times \mathbb{R})$ в том и только том случае, когда существует комплексная борелевская мера $\mu$ такая, что $h=\mathscr{F} \mu$. При этом $\|A\|_{\mathfrak{M}(\mathbb{R} \times \mathbb{R})}=\|\mu\|_{\mathcal{M}(\mathbb{R})}$.

\section{3. Двойные операторные интегралы}

Двойные операторные интегралы - это выражения вида

$$
\int_{\mathcal{S}} \int_{\mathcal{T}} \Phi(s, t) d E_{1}(s) T d E_{2}(t)
$$

где $E_{1}$ и $E_{2}$ - спектральные меры в сепарабельном гильбертовом пространстве $\mathscr{H}, \Phi$ - ограниченная измеримая функция, а $T$ - линейный непрерывный оператор в $\mathscr{H}$.

Двойные операторные интегралы появились в [23]. В работах М.Ш. Бирмана и М. З. Соломяка [19]-[21] была создана стройная теория двойных операторных интегралов. Идея Бирмана и Соломяка состояла в том, чтобы сначала определить интегралы вида (2.3.1) для произвольных ограниченных измеримых функций $\Phi$ и для операторов $T$ класса Гильберта-Шмидта $\boldsymbol{S}_{2}$. Для этого рассматривается спектральная мера $\mathscr{E}$, которая принимает значение в множестве ортогональных проекторов в гильбертовом пространстве $\boldsymbol{S}_{2}$ и определяется равенством

$$
\mathscr{E}(\Lambda \times \Delta) T=E_{1}(\Lambda) T E_{2}(\Delta), \quad T \in \boldsymbol{S}_{2},
$$

где $\Lambda$ и $\Delta$ - измеримые подмножества множеств $\mathcal{S}$ и $\mathcal{T}$. Ясно, что умножение слева на $E_{1}(\Lambda)$ коммутирует с умножением справа на $E_{2}(\Delta)$. В работе [22] было доказано, что $\mathscr{E}$ продолжается до спектральной меры на $\mathcal{S} \times \mathcal{T}$. В этой ситуации двойной операторный интеграл (2.3.1) определяется равенством

$$
\int_{\mathcal{S}} \int_{\mathcal{T}} \Phi(s, t) d E_{1}(s) T d E_{2}(t) \stackrel{\text { def }}{=}\left(\int_{\mathcal{S} \times \mathcal{T}} \Phi d \mathscr{E}\right) T .
$$


Из этого определения сразу же вытекает, что

$$
\left\|\int_{\mathcal{S}} \int_{\mathcal{T}} \Phi(s, t) d E_{1}(s) T d E_{2}(t)\right\|_{S_{2}} \leqslant\|\Phi\|_{L^{\infty}}\|T\|_{S_{2}} .
$$

Если функция $\Phi$ обладает свойством

$$
T \in \boldsymbol{S}_{1} \Rightarrow \int_{\mathcal{S}} \int_{\mathcal{T}} \Phi(s, t) d E_{1}(s) T d E_{2}(t) \in \boldsymbol{S}_{1},
$$

то говорят, что $\Phi$ - мулътипликатор Шура пространства $\boldsymbol{S}_{1}$ относителъно спектральных мер $E_{1}$ u $E_{2}$.

Чтобы определить двойные операторные интегралы (2.3.1) для ограниченных операторов $T$, рассмотрим трансформатор

$$
Q \mapsto \int_{\mathcal{T}} \int_{\mathcal{S}} \Phi(t, s) d E_{2}(t) Q d E_{1}(s), \quad Q \in \boldsymbol{S}_{1},
$$

и предположим, что функция $(y, x) \mapsto \Phi(y, x)$ - мультипликатор Шура пространства $\boldsymbol{S}_{1}$ относительно $E_{2}$ и $E_{1}$. В этом случае трансформатор

$$
T \mapsto \int_{\mathcal{S}} \int_{\mathcal{T}} \Phi(s, t) d E_{1}(s) T d E_{2}(t), \quad T \in \boldsymbol{S}_{2},
$$

продолжается по двойственности до ограниченного линейного трансформатора в пространстве ограниченных линейных операторов в $\mathscr{H}$. При этом говорят, что $\Phi$ - мультипликатор Шура (относительно $E_{1}$ u $E_{2}$ ) пространства ограниченных линейных операторов. Обозначим пространство таких мультипликаторов Шура символом $\mathfrak{M}\left(E_{1}, E_{2}\right)$. Норма функции $\Phi$ в $\mathfrak{M}\left(E_{1}, E_{2}\right)$ определяется как норма трансформатора (2.3.2) в пространстве ограниченных линейных операторов.

Легко видеть, что если функция $\Phi$ на $\mathcal{S} \times \mathcal{T}$ входит в проективное тензорное произведение $L^{\infty}\left(E_{1}\right) \widehat{\otimes} L^{\infty}\left(E_{2}\right)$ пространств $L^{\infty}\left(E_{1}\right)$ и $L^{\infty}\left(E_{2}\right)$ (т. е. допускает представление $\Phi(s, t)=\sum_{n \geqslant 0} \varphi_{n}(s) \psi_{n}(t)$, где $\left.\sum_{n \geqslant 0}\left\|\varphi_{n}\right\|_{L^{\infty}\left(E_{1}\right)}\left\|\psi_{n}\right\|_{L^{\infty}\left(E_{2}\right)}<\infty\right)$, то $\Phi \in \mathfrak{M}\left(E_{1}, E_{2}\right)$. Для таких функций $\Phi$ имеем

$$
\int_{\mathcal{S}} \int_{\mathcal{T}} \Phi(s, t) d E_{1}(s) T d E_{2}(t)=\sum_{n \geqslant 0}\left(\int_{\mathcal{S}} \varphi_{n} d E_{1}\right) T\left(\int_{\mathcal{T}} \psi_{n} d E_{2}\right) .
$$

Более общо, $\Phi \in \mathfrak{M}\left(E_{1}, E_{2}\right)$, если $\Phi$ принадлежит интегралъному проективному тензорному произведению $L^{\infty}\left(E_{1}\right) \widehat{\otimes}_{\mathrm{i}} L^{\infty}\left(E_{2}\right)$ пространств $L^{\infty}\left(E_{1}\right)$ и $L^{\infty}\left(E_{2}\right)$, т. е. $\Phi$ допускает представление

$$
\Phi(s, t)=\int_{\Omega} \varphi(s, w) \psi(t, w) d \sigma(w),
$$

где $(\Omega, \sigma)$ - пространство с $\sigma$-конечной мерой, $\varphi$ - измеримая функция на $\mathcal{S} \times \Omega$, $\psi$ - измеримая функция на $\mathcal{T} \times \Omega$ и

$$
\int_{\Omega}\|\varphi(\cdot, w)\|_{L^{\infty}\left(E_{1}\right)}\|\psi(\cdot, w)\|_{L^{\infty}\left(E_{2}\right)} d \sigma(w)<\infty .
$$


Оказывается, что все мультипликаторы Шура могут быть получены таким образом (см. теорему 2.3.1 ниже).

Ещё одно достаточное условие для того, чтобы функция была мультипликатором Шура, может быть сформулировано в терминах тензорного произведения Хогерупа $L^{\infty}\left(E_{1}\right) \widehat{\otimes}_{\mathrm{h}} L^{\infty}\left(E_{2}\right)$, которое определяется как пространство функций $\Phi$ вида

$$
\Phi(s, t)=\sum_{n \geqslant 0} \varphi_{n}(s) \psi_{n}(t),
$$

где $\left\{\varphi_{n}\right\}_{n \geqslant 0} \in L_{E_{1}}^{\infty}\left(\ell^{2}\right)$ и $\left\{\psi_{n}\right\}_{n \geqslant 0} \in L_{E_{2}}^{\infty}\left(\ell^{2}\right)$. При этом

$$
\|\Phi\|_{L^{\infty}\left(E_{1}\right) \widehat{\otimes}_{\mathrm{h}} L^{\infty}\left(E_{2}\right)} \stackrel{\text { def }}{=} \inf \left\|\sum_{n \geqslant 0}\left|\varphi_{n}\right|^{2}\right\|_{L^{\infty}\left(E_{1}\right)}^{1 / 2}\left\|\sum_{n \geqslant 0}\left|\psi_{n}\right|^{2}\right\|_{L^{\infty}\left(E_{2}\right)}^{1 / 2},
$$

где инфимум берётся по всем представлениям функции $\Phi$ вида (2.3.4). Нетрудно проверить, что если $\Phi \in L^{\infty}\left(E_{1}\right) \widehat{\otimes}_{\mathrm{h}} L^{\infty}\left(E_{2}\right)$, то $\Phi \in \mathfrak{M}\left(E_{1}, E_{2}\right)$ и

$$
\iint \Phi(s, t) d E_{1}(s) T d E_{2}(t)=\sum_{n \geqslant 0}\left(\int \varphi_{n} d E_{1}\right) T\left(\int \psi_{n} d E_{2}\right)
$$

причём ряд в правой части сходится в слабой операторной топологии и

$$
\|\Phi\|_{\mathfrak{M}\left(E_{1}, E_{2}\right)} \leqslant\|\Phi\|_{L^{\infty}\left(E_{1}\right)} \otimes_{\mathrm{h}} L^{\infty}\left(E_{2}\right) .
$$

Как видно из следующей теоремы, условие $\Phi \in L^{\infty}\left(E_{1}\right) \widehat{\otimes}_{\mathrm{h}} L^{\infty}\left(E_{2}\right)$ не только достаточно, но и необходимо.

ТеОрема 2.3.1. Пусть $\Phi$ - измеримая функиия на $\mathcal{S} \times \mathcal{T}$, и пусть $\mu$ и $\nu-$ положительные $\sigma$-конечные меры на $\mathcal{S}$ и $\mathcal{T}$, которые взаимно абсолютно непрерывны с мерами $E_{1}$ и $E_{2}$. Следуюшие условия эквивалентны:

(a) $\Phi \in \mathfrak{M}\left(E_{1}, E_{2}\right)$;

(b) $\Phi \in L^{\infty}\left(E_{1}\right) \widehat{\otimes}_{\mathrm{i}} L^{\infty}\left(E_{2}\right)$;

(c) $\Phi \in L^{\infty}\left(E_{1}\right) \widehat{\otimes}_{\mathrm{h}} L^{\infty}\left(E_{2}\right)$;

(d) существуют б-конечная мера $\sigma$ на множестве $\Omega$, измеримые функиии чна $\mathcal{S} \times \Omega$ и н на $\mathcal{T} \times \Omega$ такие, ито имеет место (2.3.3) и

$$
\left\|\left(\int_{\Omega}|\varphi(\cdot, w)|^{2} d \sigma(w)\right)^{1 / 2}\right\|_{L^{\infty}\left(E_{1}\right)}\left\|\left(\int_{\Omega}|\psi(\cdot, w)|^{2} d \sigma(w)\right)^{1 / 2}\right\|_{L^{\infty}\left(E_{2}\right)}<\infty
$$

(е) если интегральный оператор $f \mapsto \int k(x, y) f(y) d \nu(y)$ из $L^{2}(\nu)$ в $L^{2}(\mu)$ входит в класс $\boldsymbol{S}_{1}$, то в этот же класс входит и интегральный оператор $f \mapsto \int \Psi(x, y) k(x, y) f(y) d \nu(y)$.

Импликации $(\mathrm{d}) \Rightarrow(\mathrm{a}) \Leftrightarrow($ е) установлены в [21]. В случае матричных мультипликаторов Шура импликация $(\mathrm{a}) \Rightarrow(\mathrm{b})$ доказана в работе [16]. Мы отсылаем читателя к работе [56] за доказательством эквивалентности утверждений (a), (b) и (d). Эквивалентность (c) и (d) доказывается элементарно.

Легко видеть, что условия (а)-(е) эквивалентны и тому, что функция $\Phi$ является мультипликатором Шура класса $\boldsymbol{S}_{1}$.

Отметим, что с таким же успехом можно определить двойные операторные интегралы вида (2.3.1), в которых $E_{1}$ и $E_{2}$ - спектральные меры в разных гильбертовых пространствах, а $T$ - оператор из одного пространства в другое. 
ЗАмЕчАНИЕ. Из теорем 2.2.6 и 2.3.1 с лёгкостью вытекает, что если $\mathcal{S}$ и $\mathcal{T}$ - топологические пространства, а $\Phi$ - борелевская функция на $\mathcal{S} \times \mathcal{T}$ класса $\mathfrak{M}(\mathcal{S} \times \mathcal{T})\left(\right.$ т. е. $\Phi$ - дискретный мультипликатор Шура), то $\Phi \in \mathfrak{M}\left(E_{1}, E_{2}\right)$ для любых борелевских спектральных мер $E_{1}$ и $E_{2}$ на $\mathcal{S}$ и $\mathcal{T}$.

Двойные операторные интегралы могут быть определены и по отношению к полуспектральным мерам. Напомним, что полуспектральная мера $\mathcal{E}$ на измеримом пространстве $(\mathscr{X}, \mathfrak{B})$ - это отображение, заданное на $\sigma$-алгебре $\mathfrak{B}$, принимающее значения в множестве ограниченных операторов в гильбертовом пространстве $\mathscr{H}$, счётно аддитивное в сильной операторной топологии и такое, что

$$
\mathcal{E}(\Delta) \geqslant \mathbf{0}, \quad \Delta \in \mathfrak{B}, \quad \mathcal{E}(\varnothing)=\mathbf{0}, \quad \mathcal{E}(\mathscr{X})=I .
$$

По теореме Наймарка [46] всякая полуспектральная мера $\mathcal{E}$ имеет спектральную дилатацию, т. е. спектральную меру $E$, заданную на том же измеримом пространстве $(\mathscr{X}, \mathfrak{B})$, принимающую значения в множестве ортогональных проекторов в гильбертовом пространстве $\mathscr{K}$, содержащем $\mathscr{H}$, и такую, что

$$
\mathcal{E}(\Delta)=P_{\mathscr{H}} E(\Delta) \mid \mathscr{H}, \quad \Delta \in \mathfrak{B},
$$

где $P_{\mathscr{H}}$ - ортогональный проектор в $\mathscr{K}$ на подпространство $\mathscr{H}$. Такую спектральную дилатацию можно выбрать минималъной в том смысле, что

$$
\mathscr{K}=\operatorname{clos} \operatorname{span}\{E(\Delta) \mathscr{H}: \Delta \in \mathfrak{B}\} .
$$

Отметим, что в [44] показано, что если $E$ - минимальная спектральная дилатация полуспектральной меры $\mathscr{E}$, то $E$ и $\mathscr{E}$ взаимно абсолютно непрерывны.

Интегралы по полуспектральным мерам определяются следующим образом:

$$
\int_{\mathscr{X}} \varphi(x) d \mathcal{E}(x)=P_{\mathscr{H}}\left(\int_{\mathscr{X}} \varphi(x) d E(x)\right) \mid \mathscr{H}, \quad \varphi \in L^{\infty}(\mathcal{E}) \stackrel{\text { def }}{=} L^{\infty}(E) .
$$

Если $\mathcal{E}_{1}$ и $\mathcal{E}_{2}$ - полуспектральные меры на $\left(\mathscr{X}_{1}, \mathfrak{B}_{1}\right)$ и $\left(\mathscr{X}_{2}, \mathfrak{B}_{2}\right), E_{1}$ и $E_{2}-$ их спектральные дилатации в гильбертовых пространствах $\mathscr{K}_{1}$ и $\mathscr{K}_{2}$, а функция $\Phi$ на $\mathscr{X}_{1} \times \mathscr{X}_{2}$ удовлетворяет одному из условий теоремы 2.3.1, то двойной операторный интеграл по полуспектральным мерам $\mathcal{E}_{1}$ и $\mathcal{E}_{2}$ определяется равенством

$$
\begin{aligned}
& \int_{\mathscr{X}_{1}} \int_{\mathscr{X}_{2}} \Phi\left(x_{1}, x_{2}\right) d \mathcal{E}_{1}\left(x_{1}\right) Q d \mathcal{E}_{2}\left(x_{2}\right) \\
&=P_{\mathscr{H}}^{[1]} \int_{\mathscr{X}_{1}} \int_{\mathscr{X}_{2}} \Phi\left(x_{1}, x_{2}\right) d E_{1}\left(x_{1}\right)\left(Q P_{\mathscr{H}}^{[2]}\right) d E_{2}\left(x_{2}\right) \mid \mathscr{H}
\end{aligned}
$$

для произвольного ограниченного оператора $Q$ в $\mathscr{H}$. Здесь $P_{\mathscr{H}}^{[1]}$ и $P_{\mathscr{H}}^{[2]}-$ ортогональные проекторы из $\mathscr{K}_{1}$ и $\mathscr{K}_{2}$ на $\mathscr{H}$. Если $\Phi \in L^{\infty}\left(E_{1}\right) \otimes_{\mathrm{h}} L^{\infty}\left(E_{2}\right)$, то имеет место равенство

$$
\iint \Phi\left(x_{1}, x_{2}\right) d \mathcal{E}_{1}\left(x_{1}\right) T d \mathcal{E}_{2}\left(x_{2}\right)=\sum_{n \geqslant 0}\left(\int \varphi_{n} d \mathcal{E}_{1}\right) T\left(\int \psi_{n} d \mathcal{E}_{2}\right),
$$

где $T \in \mathcal{B}(\mathscr{H})$, а $\varphi_{n}$ и $\psi_{n}$ - функции из представления (2.3.4).

Двойные операторные интегралы по полуспектральным мерам были введены в работе [57] (см. также [62]). 


\section{Глава III. Операторно липшицевы функции на подмножествах плоскости}

В этой главе мы изучаем операторно липшицевы и коммутаторно липшицевы функции на замкнутых подмножествах комплексной плоскости. При этом значительную роль играют мультипликаторы Шура. Мы предлагаем два метода разностных и коммутаторных оценок. Первый метод использует дискретные мультипликаторы Шура и аппроксимацию операторами с конечным спектром. Второй метод основан на применении двойных операторных интегралов.

\section{1. Операторно липшицевы и коммутаторно липшицевы функции на замкнутых подмножествах плоскости}

Мы определим здесь классы операторно липшицевых функций и коммутаторно липшицевых функций на замкнутых подмножествах плоскости. Мы увидим, что, в отличие от случая функций на прямой и на окружности, эти два класса отнюдь не обязаны совпадать. При определении этих классов будем здесь рассматривать только ограниченные операторы. В следующем разделе мы увидим, что рассмотрение не обязательно ограниченных операторов приводит к тем же классам функций.

Пусть $\mathfrak{F}$ - непустое подмножество комплексной плоскости $\mathbb{C}$. Обозначим символом $\operatorname{Lip}(\mathfrak{F})$ пространство функций $f: \mathfrak{F} \rightarrow \mathbb{C}$, удовлетворяющих условию Липшица:

$$
|f(z)-f(w)| \leqslant C|z-w|, \quad z, w \in \mathbb{C} .
$$

Наименьшую из констант $C \geqslant 0$, удовлетворяющих условию (3.1.1), обозначим через $\|f\|_{\operatorname{Lip}(\mathfrak{F})}=\|f\|_{\text {Lip }}$. Положим $\|f\|_{\text {Lip }} \stackrel{\text { def }}{=} \infty$, если $f \notin \operatorname{Lip}(\mathfrak{F})$.

Обычно мы будем требовать, чтобы множество $\mathfrak{F}$ было замкнутым.

Из спектральной теоремы для пары коммутирующих нормальных операторов легко следует, что неравенство

$$
\left\|f\left(N_{1}\right)-f\left(N_{2}\right)\right\| \leqslant\|f\|_{\operatorname{Lip}(\mathfrak{F})}\left\|N_{1}-N_{2}\right\|
$$

выполняется для любых коммутирующих нормальных операторов $N_{1}$ и $N_{2}$, спектры которых содержатся в $\mathfrak{F}$.

Комплексную непрерывную функцию $f$, заданную на непустом замкнутом множестве $\mathfrak{F} \subset \mathbb{C}$, будем называть операторно липшицевой, если существует положительное число $C$ такое, что

$$
\left\|f\left(N_{1}\right)-f\left(N_{2}\right)\right\| \leqslant C\left\|N_{1}-N_{2}\right\|
$$

для любых нормальных операторов $N_{1}$ и $N_{2}$ со спектрами, лежащими в $\mathfrak{F}$. Пространство всех операторно липшицевых функций на $\mathfrak{F}$ обозначим символом $\mathrm{OL}(\mathfrak{F})$. Наименьшую из констант $C$, удовлетворяющих условию (3.1.3), обозначим через $\|f\|_{\mathrm{OL}(\mathfrak{F})}=\|f\|_{\mathrm{OL}}$. Положим $\|f\|_{\mathrm{OL}}=\infty$, если $f \notin \mathrm{OL}(\mathfrak{F})$.

Если функция $f$ задана на более широком множестве $\mathfrak{G} \supset \mathfrak{F}$, то для краткости мы будем обычно писать $f \in \mathrm{OL}(\mathfrak{F})$ и $\|f\|_{\mathrm{OL}(\mathfrak{F})}$ вместо $f \mid \mathfrak{F} \in \mathrm{OL}(\mathfrak{F})$ и $\|f \mid \mathfrak{F}\|_{\mathrm{OL}(\mathfrak{F})}$. Это же соглашение будет применяться и для других функциональных пространств. 
Легко видеть, что $\mathrm{OL}(\mathfrak{F}) \subset \operatorname{Lip}(\mathfrak{F})$ и $\|f\|_{\operatorname{Lip}(\mathfrak{F})} \leqslant\|f\|_{\mathrm{OL}(\mathfrak{F})}$ для любой функции $f \in \mathrm{OL}(\mathfrak{F})$. Как будет показано в разделе 3.14, равенство $\mathrm{OL}(\mathfrak{F})=\operatorname{Lip}(\mathfrak{F})$ выполняется только для конечных множеств $\mathfrak{F}$.

Заметим, что если $f \in \mathrm{OL}(\mathfrak{F})$ и $\|f\|_{\mathrm{OL}} \leqslant 1$, то

$$
\left\|f\left(N_{1}\right) U-U f\left(N_{2}\right)\right\| \leqslant\left\|N_{1} U-U N_{2}\right\|
$$

для всех унитарных операторов $U$ и всех нормальных операторов $N_{1}$ и $N_{2}$ таких, что $\sigma\left(N_{1}\right), \sigma\left(N_{2}\right) \subset \mathfrak{F}$. Чтобы убедиться в этом, достаточно применить неравенство (3.1.3) с константой $C=1 \mathrm{k}$ нормальным операторам $U^{*} N_{1} U$ и $N_{2}$. Обратно, если неравенство (3.1.4) выполняется для всех унитарных операторов $U$ и всех нормальных операторов $N_{1}$ и $N_{2}$ таких, что $N_{1}=N_{2}$ и $\sigma\left(N_{1}\right)=\sigma\left(N_{2}\right) \subset \mathfrak{F}$, то $f \in \mathrm{OL}(\mathfrak{F})$ и $\|f\|_{\mathrm{OL}(\mathfrak{F})} \leqslant 1$. Действительно, применяя неравенство (3.1.4) к операторам $\mathcal{N}_{1}=\mathcal{N}_{2}=\left(\begin{array}{cc}N_{1} & \mathbf{0} \\ \mathbf{0} & N_{2}\end{array}\right)$ и $\mathcal{U}=\left(\begin{array}{ll}\mathbf{0} & I \\ I & \mathbf{0}\end{array}\right)$, получаем: $\left\|f\left(N_{1}\right)-f\left(N_{2}\right)\right\| \leqslant\left\|N_{1}-N_{2}\right\|$. Заметим, что в этом рассуждении мы обошлись только самосопряжёнными унитарными операторами $\mathcal{U}$, т. е. нормальными операторами $\mathcal{U}$ такими, что $\mathcal{U}^{2}=I$, или, что то же самое, унитарными операторами со спектрами в $\{-1,1\}$.

Теорема 3.1.1. Пусть $f$ - непрерывная функиия на замкнутом подмножестве $\mathfrak{F}$ комплексной плоскости $\mathbb{C}$. Тогда следующие условия равносильны:

(а) $\left\|f\left(N_{1}\right)-f\left(N_{2}\right)\right\| \leqslant\left\|N_{1}-N_{2}\right\|$ для всех нормальных операторов $N_{1}$ u $N_{2}$ makux, иmo $\sigma\left(N_{1}\right), \sigma\left(N_{2}\right) \subset \mathfrak{F}$

(b) $\left\|f\left(N_{1}\right) U-U f\left(N_{2}\right)\right\| \leqslant\left\|N_{1} U-U N_{2}\right\|$ для всех унитарных операторов $U$ и всех нормальных операторов $N_{1}$ u $N_{2}$ таких, что $\sigma\left(N_{1}\right), \sigma\left(N_{2}\right) \subset \mathfrak{F}$;

(с) $\|f(N) U-U f(N)\| \leqslant\|N U-U N\|$ для всех самосопряжённых унитарных операторов $U$ и всех нормальных операторов $N$ таких, что $\sigma(N) \subset \mathfrak{F}$;

(d) $\|f(N) A-A f(N)\| \leqslant\|N A-A N\|$ для всех самосопряжённых операторов $A$ и всех нормальных операторов $N$ таких, что $\sigma(N) \subset \mathfrak{F}$.

Доказательство. Эквивалентность условий (a), (b) и (c) по существу была доказана перед формулировкой теоремы. Импликация $(\mathrm{d}) \Rightarrow(\mathrm{c})$ очевидна. Остаётся доказать, что (c) влечёт (d). Обозначим через $\mathfrak{X}$ множество всех операторов $R$ таких, что $\|f(N) R-R f(N)\| \leqslant\|N R-R N\|$ для всех нормальных операторов $N$ со спектром в $\mathfrak{F}$. Ясно, что множество $\mathfrak{X}$ замкнуто по норме и $\alpha U+\beta I \in \mathfrak{X}$ для всех унитарных операторов $U$ и всех $\alpha, \beta \in \mathbb{C}$. Теперь, чтобы доказать, что любой самосопряжённый оператор $A$ принадлежит множеству $\mathfrak{X}$, достаточно заметить, что оператор $(I-\varepsilon i A)(I+\varepsilon \mathrm{i} A)^{-1}$ является унитарным при всех $\varepsilon$ из $\left(-\|A\|^{-1},\|A\|^{-1}\right)$ и

$$
A=\lim _{\varepsilon \rightarrow 0} \frac{1}{2 \varepsilon \mathrm{i}}\left(I-(I-\varepsilon \mathrm{i} A)(I+\varepsilon \mathrm{i} A)^{-1}\right) .
$$

Теорема доказана.

ЗАмечАниЕ. Унитарный оператор $U$ является самосопряжённым в том и только том случае, когда он представим в виде $U=2 P-I$, где $P$ - ортогональный проектор. Условие (c) теоремы 3.1.1 можно переписать следующим образом: $\|f(N) P-P f(N)\| \leqslant\|N P-P N\|$ для всех ортогональных проекторов $P$ и всех нормальных операторов $N$ таких, что $\sigma(N) \subset \mathfrak{F}$. 
Теорема 3.1.2. Пусть $f$ - непрерывная функиия, заданная на замкнутом подмножестве $\mathfrak{F}$ комплексной плоскости $\mathbb{C}$. Следующие условия равносильны:

(а) $\left\|f\left(N_{1}\right)-f\left(N_{2}\right)\right\| \leqslant\left\|N_{1}-N_{2}\right\|$ для всех нормальных операторов $N_{1} u N_{2}$ maкux, чmо $\sigma\left(N_{1}\right), \sigma\left(N_{2}\right) \subset \mathfrak{F}$;

(b) $\|f(N) R-R f(N)\| \leqslant \max \left\{\|N R-R N\|,\left\|N^{*} R-R N^{*}\right\|\right\}$ для всех операторов $R \in \mathcal{B}(\mathscr{H})$ и всех нормальных операторов $N$ таких, что $\sigma(N) \subset \mathfrak{F}$;

(c) $\left\|f\left(N_{1}\right) R-R f\left(N_{2}\right)\right\| \leqslant \max \left\{\left\|N_{1} R-R N_{2}\right\|,\left\|N_{1}^{*} R-R N_{2}^{*}\right\|\right\}$ для всеx oneраторов $R \in \mathcal{B}(\mathscr{H})$ и всех нормальных операторов $N_{1}$ u $N_{2}$ таких, что $\sigma\left(N_{1}\right), \sigma\left(N_{2}\right) \subset \mathfrak{F}$.

ДокАзАтельство. Докажем сначала импликацию $(\mathrm{a}) \Rightarrow(\mathrm{b})$. Пусть выполнено условие (а). Тогда из теоремы 3.1.1 следует, что $\|f(N) A-A f(N)\| \leqslant$ $\|N A-A N\|$ для всех самосопряжённых операторов $A$ и всех нормальных операторов $N$ таких, что $\sigma(N) \subset \mathfrak{F}$. Применяя это утверждение к нормальному оператору $\left(\begin{array}{cc}N & \mathbf{0} \\ \mathbf{0} & N\end{array}\right)$ и самосопряжённому оператору $\left(\begin{array}{cc}\mathbf{0} & R \\ R^{*} & \mathbf{0}\end{array}\right)$, получим

$$
\begin{aligned}
\max \left\{\|f(N) R-R f(N)\|,\left\|f(N) R^{*}-R^{*} f(N)\right\|\right\} & \\
& \left.\leqslant \max \{\| N R-R N)\|,\| N R^{*}-R^{*} N \|\right\},
\end{aligned}
$$

откуда следует (b).

Применяя (b) к нормальному оператору $\left(\begin{array}{cc}N_{1} & \mathbf{0} \\ \mathbf{0} & N_{2}\end{array}\right)$ и оператору $\left(\begin{array}{cc}\mathbf{0} & R \\ \mathbf{0} & \mathbf{0}\end{array}\right)$, получим (c). Импликация (c) $\Rightarrow$ (а) очевидна. Теорема доказана.

В доказательствах теорем 3.1.1 и 3.1.2 мы использовали стандартный приём, связанный с переходом к блочным операторам, который позволяет в некоторых случаях переходить от одного оператора к паре операторов. Этот приём будет полезен и в дальнейшем. Киттанех [40] называет его трюком Бербериана, имея в виду, видимо, статью Бербериана [17].

Теоремы 3.1.1 и 3.1.2 содержатся в теореме 3.1 статьи [13], но по существу в некотором виде их можно извлечь из статьи [38], где наряду с операторной нормой рассматриваются также произвольные симметричные нормы.

Заметим, что равенство $\left\|N_{1}^{*} R-R N_{2}^{*}\right\|=\left\|N_{1} R-R N_{2}\right\|$, а значит, и равенство

$$
\max \left\{\left\|N_{1} R-R N_{2}\right\|,\left\|N_{1}^{*} R-R N_{2}^{*}\right\|\right\}=\left\|N_{1} R-R N_{2}\right\|,
$$

выполняется в каждом из следующих частных случаев:

1) операторы $N_{1}$ и $N_{2}$ являются самосопряжёнными (это так, если $\mathfrak{F} \subset \mathbb{R}$ );

2) операторы $N_{1}$ и $N_{2}$ являются унитарными (это так, если $\mathfrak{F} \subset \mathbb{T}$ );

3) $R$ - самосопряжённый оператор и $N_{1}=N_{2}$;

4) $R$ - унитарный оператор.

Комплексную функцию $f$, непрерывную на замкнутом множестве $\mathfrak{F} \subset \mathbb{C}$, будем называть коммутаторно липшищевой функцией, если существует число $C \geqslant 0$ такое, что

$$
\|f(N) R-R f(N)\| \leqslant C\|N R-R N\|
$$

для всех $R \in \mathcal{B}(\mathscr{H})$ и всех нормальных операторов $N$ со спектром в $\mathfrak{F}$. Множество всех коммутаторно липшицевых функций, заданных на $\mathfrak{F}$, обозначим символом $\mathrm{CL}(\mathfrak{F})$. Наименьшую из констант $C$, удовлетворяющих условию (3.1.5), обозначим через $\|f\|_{\mathrm{CL}(\mathfrak{F})}=\|f\|_{\mathrm{CL}}$. Положим $\|f\|_{\mathrm{CL}(\mathfrak{F})}=\infty$, если $f \notin \mathrm{CL}(\mathfrak{F})$. 
Теорема 3.1.3. Пусть $f$-непрерывная функиия, заданная на замкнутом подмножестве $\mathfrak{F}$ комплексной плоскости $\mathbb{C}$. Тогда следующие три условия равносильны:

(а) $\|f(N) R-R f(N)\| \leqslant\|N R-R N\|$ для всех операторов $R \in \mathcal{B}(\mathscr{H})$ и всех нормальных операторов $N$ таких, что $\sigma(N) \subset \mathfrak{F}$;

(b) $\left\|f\left(N_{1}\right) R-R f\left(N_{2}\right)\right\| \leqslant\left\|N_{1} R-R N_{2}\right\|$ для всех операторов $R \in \mathcal{B}(\mathscr{H})$ $u$ всех нормальных операторов $N_{1}$ u $N_{2}$ таких, что $\sigma\left(N_{1}\right), \sigma\left(N_{2}\right) \subset \mathfrak{F}$;

(c) $\left\|f\left(N_{1}\right) A-A f\left(N_{2}\right)\right\| \leqslant\left\|N_{1} A-A N_{2}\right\|$ для всех самосопряжённых операторов $A$ и всех нормальных операторов $N_{1}$ и $N_{2}$ mаких, что $\sigma\left(N_{1}\right), \sigma\left(N_{2}\right) \subset \mathfrak{F}$.

ДокАзАтЕльство. Чтобы доказать импликацию $(\mathrm{a}) \Rightarrow(\mathrm{b})$, достаточно применить (а) к нормальному оператору $\left(\begin{array}{cc}N_{1} & \mathbf{0} \\ \mathbf{0} & N_{2}\end{array}\right)$ и оператору $\left(\begin{array}{cc}\mathbf{0} & R \\ \mathbf{0} & \mathbf{0}\end{array}\right)$. Импликация (b) $\Rightarrow$ (c) очевидна. Остаётся доказать, что (c) влечёт (a). Применяя (c) к $N_{1}=U^{*} N U$ и $N_{2}=N$, где $U$ - унитарный оператор, получаем:

$$
\|f(N) U A-U A f(N)\|=\left\|f\left(U^{*} N U\right) A-A f(N)\right\| \leqslant\|N U A-U A N\|
$$

для любых самосопряжённого оператора $A$, унитарного оператора $U$ и нормального оператора $N$ такого, что $\sigma(N) \subset \mathfrak{F}$. Заметим, что если условие (а) выполняется для оператора $R \in \mathcal{B}(\mathscr{H})$, то оно будет выполняться и для оператора $R+\lambda I$, где $\lambda \in \mathbb{C}$. Таким образом, можно считать, что оператор $R$ обратим. Тогда, применяя полярное разложение к обратимому оператору $R$, получаем: $R=U A$, где $U$ - унитарный оператор, $A-$ (положительный) самосопряжённый оператор. Теорема доказана.

Из теорем 3.1.1 и 3.1.3 мгновенно следует, что $\mathrm{CL}(\mathfrak{F}) \subset \mathrm{OL}(\mathfrak{F})$ и $\|f\|_{\mathrm{OL}(\mathfrak{F})} \leqslant$ $\|f\|_{\mathrm{CL}(\mathfrak{F})}$ для всех $f$ из $\mathrm{CL}(\mathfrak{F})$.

ЗАмечАниЕ. В условиях (b) теоремы 3.1.1, (c) теоремы 3.1 .2 и (b) теоремы 3.1.3 можно считать, что нормальные операторы $N_{1}$ и $N_{2}$ действуют в разных гильбертовых пространствах (при этом унитарный оператор $U$ может действовать из одного гильбертова пространства в другое). Это видно из доказательств. В качестве иллюстрации мы приведём здесь соответствующую переформулировку утверждения (b) теоремы 3.1.3: $\left\|f\left(N_{1}\right) R-R f\left(N_{2}\right)\right\| \leqslant$ $\left\|N_{1} R-R N_{2}\right\|$ для всех операторов $R \in \mathcal{B}\left(\mathscr{H}_{2}, \mathscr{H}_{1}\right)$ и всех нормальных операторов $N_{1}$ и $N_{2}$, действующих соответственно в гилъбертовых пространствах $\mathscr{H}_{1}$ и $\mathscr{H}_{2}$ и удовлетворяюших условию $\sigma\left(N_{1}\right), \sigma\left(N_{2}\right) \subset \mathfrak{F}$.

Аналоги теорем 3.1.1-3.1.3 с соответствующими изменениями имеют место по существу с теми же доказательствами для симметрично-нормированных идеалов. Мы рассмотрим здесь только идеал ядерных операторов $\boldsymbol{S}_{1}$. С каждым замкнутым множеством $\mathfrak{F} \subset \mathbb{C}$ мы связываем пространство ядерно липиицевъх (или $\boldsymbol{S}_{1}$-липшицевых) функций $\mathrm{OL}_{\boldsymbol{S}_{1}}(\mathfrak{F})$ и ядерно-коммутаторно липшицевых (или $\boldsymbol{S}_{1}$-коммутаторно липшицевых) функций $\mathrm{CL}_{\boldsymbol{S}_{1}}(\mathfrak{F})$. Чтобы получить определение пространств $\mathrm{OL}_{\boldsymbol{S}_{1}}(\mathfrak{F})$ и $\mathrm{CL}_{\boldsymbol{S}_{1}}(\mathfrak{F})$, нужно только в неравенствах (3.1.3) и (3.1.5) операторную норму заменить ядерной нормой.

Соответствующий “объединённый” аналог теорем 3.1.1 и 3.1.2 для ядерной нормы может быть сформулирован следующим образом. 
Теорема 3.1.4. Пусть $f$ - непрерьвная функция на замкнутом подмножестве $\mathfrak{F}$ комплексной плоскости $\mathbb{C}$. Тогда следующие условия равносильны:

(a) $\left\|f\left(N_{1}\right)-f\left(N_{2}\right)\right\|_{S_{1}} \leqslant\left\|N_{1}-N_{2}\right\|_{S_{1}}$ для всех нормалъных операторов $N_{1}$ u $N_{2}$ maкux, что $\sigma\left(N_{1}\right), \sigma\left(N_{2}\right) \subset \mathfrak{F}$;

(b) $\left\|f\left(N_{1}\right) U-U f\left(N_{2}\right)\right\|_{S_{1}} \leqslant\left\|N_{1} U-U N_{2}\right\|_{S_{1}}$ для всех унитарных операторов $U$ и всех нормальных операторов $N_{1}$ и $N_{2}$ таких, что $\sigma\left(N_{1}\right), \sigma\left(N_{2}\right) \subset \mathfrak{F}$;

(с) $\|f(N) U-U f(N)\|_{S_{1}} \leqslant\|N U-U N\|_{S_{1}}$ для всех самосопряжсённых унитарных операторов $U$ и всех нормальных операторов $N$ таких, что $\sigma(N) \subset \mathfrak{F}$;

(d) $\|f(N) A-A f(N)\|_{S_{1}} \leqslant\|N A-A N\|_{S_{1}}$ для всех самосопряжённых операторов $A$ и всех нормальных операторов $N$ таких, что $\sigma(N) \subset \mathfrak{F}$;

(e) $\|f(N) R-R f(N)\|_{S_{1}}+\|\bar{f}(N) R-R \bar{f}(N)\|_{S_{1}} \leqslant\|N R-R N\|_{S_{1}}+\| N^{*} R-$ $R N^{*} \|_{S_{1}}$ для всех операторов $R \in \mathcal{B}(\mathscr{H})$ и всех нормалъных операторов $N$ makux, чmo $\sigma(N) \subset \mathfrak{F}$;

(f) $\left\|f\left(N_{1}\right) R-R f\left(N_{2}\right)\right\|_{S_{1}}+\left\|\bar{f}\left(N_{1}\right) R-R \bar{f}\left(N_{2}\right)\right\|_{S_{1}} \leqslant\left\|N_{1} R-R N_{2}\right\|_{S_{1}}+\| N_{1}^{*} R-$ $R N_{2}^{*} \|_{S_{1}}$ для всех операторов $R \in \mathcal{B}(\mathscr{H})$ и всех нормальных операторов $N_{1}$ $u N_{2}$ maкux, ито $\sigma\left(N_{1}\right), \sigma\left(N_{2}\right) \subset \mathfrak{F}$.

Приведём теперь аналог теоремы 3.1.3.

Теорема 3.1.5. Пусть $f$ - непрерывная функция, заданная на замкнутом подмножестве $\mathfrak{F}$ комплексной плоскости $\mathbb{C}$. Тогда следующие три условия равносильны:

(а) $\|f(N) R-R f(N)\|_{S_{1}} \leqslant\|N R-R N\|_{S_{1}}$ для всех операторов $R \in \mathcal{B}(\mathscr{H})$ и всех нормальных операторов $N$ таких, что $\sigma(N) \subset \mathfrak{F}$;

(b) $\left\|f\left(N_{1}\right) R-R f\left(N_{2}\right)\right\|_{S_{1}} \leqslant\left\|N_{1} R-R N_{2}\right\|_{S_{1}}$ для всех операторов $R \in \mathcal{B}(\mathscr{H})$ и всех нормалъных операторов $N_{1}$ u $N_{2}$ mаких, ито $\sigma\left(N_{1}\right), \sigma\left(N_{2}\right) \subset \mathfrak{F}$;

(c) $\left\|f\left(N_{1}\right) A-A f\left(N_{2}\right)\right\|_{S_{1}} \leqslant\left\|N_{1} A-A N_{2}\right\|_{S_{1}}$ для всех самосопряжённых операторов $A$ и всех нормальных операторов $N_{1}$ и $N_{2}$ таких, что $\sigma\left(N_{1}\right), \sigma\left(N_{2}\right) \subset \mathfrak{F}$.

Заметим, что для самосопряжённых операторов теорему 3.1.4 можно переформулировать следующим образом.

Теорема 3.1.6. Пусть $f$ - вещественная непреръвная функиия, заданная на замкнутом подмножестве $\mathfrak{F}$ вещественной прямой $\mathbb{R}$. Тогда следующие условия равносильны:

(a) $\|f(A)-f(B)\|_{S_{1}} \leqslant\|A-B\|_{S_{1}}$ для всех самосопряжённых операторов $A$ u $B$ maкux, чmо $\sigma(A), \sigma(B) \subset \mathfrak{F}$;

(b) $\|f(A) U-U f(B)\|_{S_{1}} \leqslant\|A U-U B\|_{S_{1}}$ для всех унитарных операторов $U$ и всех самосопряжеённых операторов $A$ и $B$ таких, что $\sigma(A), \sigma(B) \subset \mathfrak{F}$;

(c) $\|f(A) U-U f(A)\|_{S_{1}} \leqslant\|A U-U A\|_{S_{1}}$ для всех самосопряжённых унитарных операторов $U$ и всех самосопряжённьх операторов $A$ таких, что $\sigma(A) \subset \mathfrak{F}$

(d) $\|f(A) R-R f(A)\|_{S_{1}} \leqslant\|A R-R A\|_{S_{1}}$ для всех самосопряжённых операторов $A$ и $R$ mаких, что $\sigma(A) \subset \mathfrak{F}$;

(е) $\|f(A) R-R f(A)\|_{S_{1}} \leqslant\|A R-R A\|_{S_{1}}$ для всех операторов $R \in \mathcal{B}(\mathscr{H})$ и всех самосопряжённых операторов $A$ таких, что $\sigma(A) \subset \mathfrak{F}$;

(f) $\|f(A) R-R f(B)\|_{S_{1}} \leqslant\|A R-R B\|_{S_{1}}$ для всех операторов $R \in \mathcal{B}(\mathscr{H}) u$ всех самосопряжённых операторов $A$ и $B$ таких, что $\sigma(A), \sigma(B) \subset \mathfrak{F}$. 
СлЕДСТвиЕ 3.1.7. Если $f$ - вещественная непрерывная функция на замкнутом подмножестве $\mathfrak{F}$ вещественной прямой $\mathbb{R}$, mо $\|f\|_{\mathrm{OL}_{S_{1}}(\mathfrak{F})}=\|f\|_{\mathrm{CL}(\mathfrak{F})}$.

Отсюда следует, что для комплексной непрерывной функции $f$ выполнены неравенства $\|f\|_{\mathrm{OL}_{S_{1}}(\mathfrak{F})} \leqslant\|f\|_{\mathrm{CL}_{S_{1}}(\mathfrak{F})} \leqslant 2\|f\|_{\mathrm{OL}_{S_{1}}(\mathfrak{F})}$.

Всё то же самое можно сказать и в случае унитарных операторов $N_{1}$ и $N_{2}$, т. е. в том случае, когда множество $\mathfrak{F}$ лежит на единичной окружности $\mathbb{T}$.

Ясно, что $\bar{z} \in \mathrm{OL}(\mathfrak{F})$ для любого замкнутого множества $\mathfrak{F}$ в $\mathbb{C}$ и $\|\bar{z}\|_{\mathrm{OL}(\mathfrak{F})}=1$, если множество $\mathfrak{F}$ содержит по крайней мере две точки.

ОПредЕЛЕНИЕ. Замкнутое подмножество $\mathfrak{F}$ комплексной плоскости $\mathbb{C}$ называется множеством Фугледе, если $\mathrm{CL}(\mathfrak{F})=\mathrm{OL}(\mathfrak{F})$.

Это понятие было введено Э. Киссиным и В. С. Шульманом в работе [38].

Б. Джонсон и Дж. Вильямс [32] доказали, что каждая функция $f \in \mathrm{CL}(\mathfrak{F})$ дифференцируема в комплексном смысле в каждой неизолированной точке множества $\mathfrak{F}$ (см. ниже теоремы 3.3 .2 и 3.3.3). Заметим, что $\bar{z} \in \mathrm{OL}(\mathfrak{F})$. Таким образом, множество Фугледе не может иметь внутренних точек и даже не может содержать двух пересекающихся отрезков, не лежащих на одной прямой. Киссин и Шульман [38] доказали, что любая компактная кривая класса $C^{2}$ является множеством Фугледе.

Следующая теорема по существу содержится в предложении 4.5 статьи [38].

ТЕОРема 3.1.8. Замкнутое подмножество $\mathfrak{F}$ комплексной плоскости $\mathbb{C}$ является множеством Фугледе в том и только том случае, когда $\bar{z} \in \mathrm{CL}(\mathfrak{F})$. Если $\bar{z} \in \mathrm{CL}(\mathfrak{F})$, mo $\|f\|_{\mathrm{CL}(\mathfrak{F})} \leqslant\|\bar{z}\|_{\mathrm{CL}(\mathfrak{F})}\|f\|_{\mathrm{OL}(\mathfrak{F})}$ для всех $f \in \mathrm{OL}(\mathfrak{F})$.

Очевидным образом эта теорема вытекает из теорем 3.1.2 и 3.1.3.

СлЕДСТВИЕ 3.1.9. Пусть $\mathfrak{F}$ - замкнутое подмножество комплексной плоскости $\mathbb{C}$. Тогда равенство $\mathrm{CL}(\mathfrak{F})=\mathrm{OL}(\mathfrak{F})$ имеет место вместе с равенством полунорм $\|\cdot\|_{\mathrm{CL}(\mathfrak{F})}=\|\cdot\|_{\mathrm{OL}(\mathfrak{F})}$ в том и только том случае, когда $\|\bar{z}\|_{\mathrm{CL}(\mathfrak{F})} \leqslant 1$.

Заметим, что $\|\bar{z}\|_{\mathrm{CL}(\mathfrak{F})} \geqslant\|\bar{z}\|_{\mathrm{OL}(\mathfrak{F})}=1$, если только множество $\mathfrak{F}$ содержит по крайней мере две точки. Таким образом, условие $\|\bar{z}\|_{\mathrm{CL}(\mathfrak{F})} \leqslant 1$ можно заменить условием $\|\bar{z}\|_{\mathrm{CL}(\mathfrak{F})}=1$, если множество $\mathfrak{F}$ содержит по крайней мере две точки.

Теорема 3.1.10. Если все точки замкнутого подмножества $\mathfrak{F}$ комплексной плоскости $\mathbb{C}$ лежат на прямой или окружности, то $\mathfrak{F}$ - множество Фугледе $и\|\cdot\|_{\mathrm{CL}(\mathfrak{F})}=\|\cdot\|_{\mathrm{OL}(\mathfrak{F})}$.

ДоказатеЛЬство. Легко видеть, что (полу)нормы в пространствах CL(F) и $\mathrm{OL}(\mathfrak{F})$ совпадают тогда и только тогда, когда

$$
\left\|N^{*} R-R N^{*}\right\|=\|N R-R N\|
$$

для всех нормальных операторов $N$ со спектром в $\mathfrak{F}$ и всех ограниченных операторов $R$. Как было отмечено выше (см. частные случаи 1) и 2) перед теоремой 3.1.3), это равенство очевидным образом выполняется для самосопряжённых операторов $N$ и унитарных операторов $N$. Это доказывает теорему в следующих двух частных случаях: $\mathfrak{F} \subset \mathbb{R}, \mathfrak{F} \subset \mathbb{T}$. Общий случай сводится к этим частным случаям при помощи аффинных преобразований комплексной плоскости. 
Г. Камовиц [33] доказал, что равенство (3.1.6) выполняется для всех ограниченных операторов $R$ при фиксированном операторе $N$ в том и только том случае, когда $N$ - нормальный оператор со спектром, лежащим на окружности или на прямой. Из этого результата Камовица мгновенно следует, что теорему 3.1.10 можно обратить. Другими словами, равенство $\|\cdot\|_{\mathrm{CL}(\mathfrak{F})}=\|\cdot\|_{\mathrm{OL}(\mathfrak{F})}$ имеет место в том и только том случае, когда множество $\mathfrak{F}$ лежит на окружности или на прямой.

Пусть $\mathfrak{F}_{1}$ и $\mathfrak{F}_{2}$ - непустые замкнутые подмножества комплексной плоскости $\mathbb{C}$. Обозначим через $\mathrm{CL}\left(\mathfrak{F}_{1}, \mathfrak{F}_{2}\right)$ пространство всех непрерывных функций $f$, заданных на $\mathfrak{F}=\mathfrak{F}_{1} \cup \mathfrak{F}_{2}$, для которых существует константа $C \geqslant 0$ такая, что

$$
\left\|f\left(N_{1}\right) R-R f\left(N_{2}\right)\right\| \leqslant C\left\|N_{1} R-R N_{2}\right\|
$$

для всех $R \in \mathcal{B}(\mathscr{H})$ и всех нормальных операторов $N_{1}$ и $N_{2}$ со спектрами, лежащими в $\mathfrak{F}_{1}$ и $\mathfrak{F}_{2}$. Наименьшую из констант $C$, удовлетворяющих условию (3.1.7), обозначим через $\|f\|_{\mathrm{CL}\left(\mathfrak{F}_{1}, \mathfrak{F}_{2}\right)}$. Положим $\|f\|_{\mathrm{CL}\left(\mathfrak{F}_{1}, \mathfrak{F}_{2}\right)}=\infty$, если $f \notin \mathrm{CL}\left(\mathfrak{F}_{1}, \mathfrak{F}_{2}\right)$.

Переходя к сопряжённым операторам, мы видим, что условие (3.1.7) равносильно условию $\left\|R^{*} \bar{f}\left(N_{1}\right)-\bar{f}\left(N_{2}\right) R^{*}\right\| \leqslant C\left\|R^{*} N_{1}^{*}-N_{2}^{*} R^{*}\right\|$. Отсюда следует, что $f \in \mathrm{CL}\left(\mathfrak{F}_{1}, \mathfrak{F}_{2}\right)$ в том и только том случае, когда $\bar{f}(\bar{z}) \in \mathrm{CL}\left(\overline{\mathfrak{F}}_{2}, \overline{\mathfrak{F}}_{1}\right)$ и $\|\bar{f}(\bar{z})\|_{\mathrm{CL}\left(\overline{\mathfrak{F}}_{2}, \overline{\mathfrak{F}}_{1}\right)}=\|f\|_{\mathrm{CL}\left(\mathfrak{F}_{1}, \mathfrak{F}_{2}\right)}$, где для подмножества $\mathfrak{F}$ комплексной плоскости $\mathbb{C}$ символом $\overline{\mathfrak{F}}$ обозначается множество $\{\bar{\zeta}: \zeta \in \mathfrak{F}\}$.

Если условие (3.1.7) переписать в терминах матриц, а затем перейти к транспонированным матрицам, то точно так же получится, что $\operatorname{CL}\left(\mathfrak{F}_{1}, \mathfrak{F}_{2}\right)=\operatorname{CL}\left(\mathfrak{F}_{2}, \mathfrak{F}_{1}\right)$ и $\|\cdot\|_{\mathrm{CL}\left(\mathfrak{F}_{1}, \mathfrak{F}_{2}\right)}=\|\cdot\|_{\mathrm{CL}\left(\mathfrak{F}_{2}, \mathfrak{F}_{1}\right)}$.

ТЕОРема 3.1.11. Пусть $f$ - непрерывная функция, заданная на оббединении $\mathfrak{F}_{1} \cup \mathfrak{F}_{2}$ замкнутых подмножеств $\mathfrak{F}_{1} u \mathfrak{F}_{2}$ комплексной плоскости $\mathbb{C}$. Тогда следуюшие условия равносильны:

(a) $\left\|f\left(N_{1}\right) R-R f\left(N_{2}\right)\right\| \leqslant\left\|N_{1} R-R N_{2}\right\|$ для всех операторов $R \in \mathcal{B}(\mathscr{H}) u$ всех нормальных операторов $N_{1}$ u $N_{2}$ таких, что $\sigma\left(N_{1}\right) \subset \mathfrak{F}_{1} u \sigma\left(N_{2}\right) \subset \mathfrak{F}_{2}$;

(b) $\left\|f\left(N_{1}\right) R-R f\left(N_{2}\right)\right\| \leqslant\left\|N_{1} R-R N_{2}\right\|$ для любого оператора $R$, действующего из произвольного гильбертова пространства $\mathscr{H}_{2}$ в произвольное гильбертово пространство $\mathscr{H}_{1}$, и всех нормальных операторов $N_{1}$ u $N_{2}$, действующих соответственно в пространствах $\mathscr{H}_{1}$ и $\mathscr{H}_{2}$ и таких, ито $\sigma\left(N_{1}\right) \subset \mathfrak{F}_{1}$ $u \sigma\left(N_{2}\right) \subset \mathfrak{F}_{2}$

(c) утверждение (b) выполняется при дополнительном предположении о простоте спектров нормалъных операторов $N_{1}$ u $N_{2}$;

(d) $\left\|f\left(N_{1}\right) A-A f\left(N_{2}\right)\right\| \leqslant\left\|N_{1} A-A N_{2}\right\|$ для всех самосопряжённых операторов $A$ и всех нормальных операторов $N_{1}$ u $N_{2}$ mаких, что $\sigma\left(N_{1}\right) \subset \mathfrak{F}_{1} u$ $\sigma\left(N_{2}\right) \subset \mathfrak{F}_{2}$.

ДокАЗАтЕльство. Импликации $(\mathrm{b}) \Rightarrow(\mathrm{a})$ и $(\mathrm{b}) \Rightarrow(\mathrm{c})$ тривиальны. Докажем, что $(\mathrm{a}) \Rightarrow(\mathrm{b})$ и $(\mathrm{c}) \Rightarrow(\mathrm{b})$. Если пространства $\mathscr{H}_{1}$ и $\mathscr{H}_{2}$ изоморфны, то существует унитарный оператор $U: \mathscr{H}_{1} \rightarrow \mathscr{H}_{2}$. Тогда оператор $R U$ и нормальные операторы $N_{1}$ и $U^{*} N_{2} U$ действуют в одном и том же гильбертовом пространстве $\mathscr{H}_{1}$, поэтому

$$
\left\|f\left(N_{1}\right)(R U)-(R U) f\left(U^{*} N_{2} U\right)\right\| \leqslant\left\|N_{1}(R U)-(R U) U^{*} N_{2} U\right\|
$$


в силу (а), откуда мгновенно следует нужная нам оценка. Чтобы свести общий случай к рассмотренному выше частному, рассмотрим операторы

$$
\mathcal{R} \stackrel{\text { def }}{=} \bigoplus_{j \geqslant 1} R, \quad \mathcal{N}_{1} \stackrel{\text { def }}{=} \bigoplus_{j \geqslant 1} N_{1} \quad \text { и } \quad \mathcal{N}_{2} \stackrel{\text { def }}{=} \bigoplus_{j \geqslant 1} N_{2} .
$$

Легко видеть, что неравенство $\left\|f\left(N_{1}\right) R-R f\left(N_{2}\right)\right\| \leqslant\left\|N_{1} R-R N_{2}\right\|$ равносильно неравенству $\left\|f\left(\mathcal{N}_{1}\right) \mathcal{R}-\mathcal{R} f\left(\mathcal{N}_{2}\right)\right\| \leqslant\left\|\mathcal{N}_{1} \mathcal{R}-\mathcal{R} \mathcal{N}_{2}\right\|$.

Докажем теперь, что (c) влечёт (b). Предположим противное. Тогда существуют оператор $R \in \mathcal{B}\left(\mathscr{H}_{2}, \mathscr{H}_{1}\right)$ и нормальные операторы $N_{1}$ и $N_{2}$, действующие соответственно в пространствах $\mathscr{H}_{1}$ и $\mathscr{H}_{2}$ и такие, что $\sigma\left(N_{1}\right) \subset \mathfrak{F}_{1}$, $\sigma\left(N_{2}\right) \subset \mathfrak{F}_{2},\left\|N_{1} R-R N_{2}\right\|=1$ и $\left\|f\left(N_{1}\right) R-R f\left(N_{2}\right)\right\|>1$. Таким образом, существуют векторы $u_{0} \in \mathscr{H}_{2}$ и $v_{0} \in \mathscr{H}_{1}$ такие, что $\left\|u_{0}\right\|=1,\left\|v_{0}\right\|=1$ и $\left|\left(\left(f\left(N_{1}\right) R-R f\left(N_{2}\right)\right) u_{0}, v_{0}\right)\right|>1$. Пусть $\mathscr{H}_{1}^{0}$ и $\mathscr{H}_{2}^{0}$ обозначают наименьшие приводящие подпространства операторов $N_{1}$ и $N_{2}$, содержащие соответственно $v_{0}$ и $u_{0}$. Пусть $P$ и $Q$ обозначают ортогональные проекторы на подпространства $\mathscr{H}_{1}^{0}$ и $\mathscr{H}_{2}^{0}$ соответственно. Заметим, что $\left\|f\left(N_{1}\right) P R Q-\operatorname{PRQf}\left(N_{2}\right)\right\|>1$, поскольку $\left(\left(f\left(N_{1}\right) P R Q-P R Q f\left(N_{2}\right)\right) u_{0}, v_{0}\right)=\left(\left(f\left(N_{1}\right) R-R f\left(N_{2}\right)\right) u_{0}, v_{0}\right)$. Kроме того, $\left\|N_{1} P R Q-P R Q N_{2}\right\|=\left\|P\left(N_{1} R-R N_{2}\right) Q\right\| \leqslant 1$. Положим $N_{1}^{0} \stackrel{\text { def }}{=} N \mid \mathscr{H}_{1}^{0}$ и $N_{2}^{0} \stackrel{\text { def }}{=} N \mid \mathscr{H}_{2}^{0}$. Тогда операторы $N_{1}^{0}$ и $N_{2}^{0}$ можно рассматривать как нормальные операторы соответственно в пространствах $\mathscr{H}_{1}^{0}$ и $\mathscr{H}_{2}^{0}$. Ясно, что $N_{1}^{0}$ и $N_{2}^{0}$ - нормальные операторы с простыми спектрами. Теперь, чтобы получить противоречие, достаточно заметить, что $\left\|f\left(N_{1}^{0}\right) P R Q-P R Q f\left(N_{2}^{0}\right)\right\|>1$ и $\left\|N_{1}^{0} P R Q-P R Q N_{2}^{0}\right\| \leqslant 1$.

Импликация $(\mathrm{a}) \Rightarrow(\mathrm{d})$ тривиальна. Остаётся доказать, что (d) влечёт (a). Применяя (d) к нормальным операторам $U^{*} N_{1} U$ и $N_{2}$, где $U$ - унитарный оператор, получаем

$$
\left\|f\left(N_{1}\right) U A-U A f\left(N_{2}\right)\right\| \leqslant\left\|N_{1} U A-U A N_{2}\right\|
$$

для любого самосопряжённого оператора $A$, унитарного оператора $U$ и нормальных операторов $N_{1}$ и $N_{2}$ таких, что $\sigma\left(N_{1}\right) \subset \mathfrak{F}_{1}$ и $\sigma\left(N_{2}\right) \subset \mathfrak{F}_{2}$. Отсюда при помощи полярного разложения следует (а) для обратимых операторов $R$. Следовательно, утверждение (а) верно для всех операторов $R$, принадлежащих замыканию по операторной норме множества всех обратимых операторов. Остаётся заметить, что в общем случае блочный оператор $\mathcal{R}=\left(\begin{array}{cc}R & \mathbf{0} \\ \mathbf{0} & \mathbf{0}\end{array}\right)$ в пространстве $\mathscr{H} \oplus \mathscr{H}$ очевидно приближается по операторной норме с любой точностью обратимыми операторами из пространства $\mathcal{B}(\mathscr{H} \oplus \mathscr{H})$. Перейти от оператора $\mathcal{R}$ к оператору $R$ можно при помощи приёма Бербериана, о котором речь шла после доказательства теоремы 3.1.2. Это завершает доказательство.

Нам понадобится следующее хорошо известное элементарное утверждение.

Лемма 3.1.12. Пусть $N$ - ограниченный нормальный оператор. Предположим, что множество $\Lambda \subset \mathbb{C}$, является $\varepsilon$-сетъю спектра $\sigma(N)$ оператоpa $N$, т.е. для любой точки $\zeta \in \sigma(N)$ существует точка $\lambda \in \Lambda$ такая, что $|\lambda-\zeta|<\varepsilon$. Тогда существует нормальный оператор $N_{0}$ такой, что $N N_{0}=N_{0} N,\left\|N-N_{0}\right\|<\varepsilon u \sigma\left(N_{0}\right)$ - конечное подмножество множества $\Lambda$. 
ДокАЗАТЕЛЬство. В силу компактности спектра оператора $N$ существует конечная $\varepsilon$-сеть $\Lambda_{0}$ множества $\sigma(N)$ такая, что $\Lambda_{0} \subset \Lambda$. Тогда мы можем найти борелевскую функцию $\eta: \sigma(N) \rightarrow \Lambda_{0}$ такую, что $\sup \{|z-\eta(z)|: z \in \sigma(N)\}<\varepsilon$. Остаётся положить $N_{0} \stackrel{\text { def }}{=} \eta(N)$. Лемма доказана.

Из этой леммы и из неравенства (3.1.2) легко вытекает, что если неравенство (3.1.3) выполняется для всех нормальных операторов $N_{1}$ и $N_{2}$ с конечными спектрами, лежащими в $\mathfrak{F}$, то $f \in \mathrm{OL}(\mathfrak{F})$ и $\|f\|_{\mathrm{OL}(\mathfrak{F})} \leqslant C$.

Другими словами, для любой непрерывной функции $f$, заданной на замкнутом множестве $\mathfrak{F} \subset \mathbb{C}$, выполняется следующее равенство:

$$
\|f\|_{\mathrm{OL}(\mathfrak{F})}=\sup \left\{\|f\|_{\mathrm{OL}(\Lambda)}: \Lambda \subset \mathfrak{F}, \Lambda \text { конечно }\right\} .
$$

Более того,

$$
\|f\|_{\mathrm{OL}(\mathfrak{F})}=\sup \left\{\|f\|_{\mathrm{OL}(\Lambda)}: \Lambda \subset \mathfrak{F}_{0}, \Lambda \text { конечно }\right\},
$$

где $\mathfrak{F}_{0}$ - всюду плотное подмножество множества $\mathfrak{F}$.

Аналогичные равенства справедливы и для коммутаторно липшицевых полунорм.

Отсюда видно, что по существу мы не получили бы ничего нового, если бы попытались определить пространства $\mathrm{OL}(\mathfrak{F})$ и $\mathrm{CL}(\mathfrak{F})$ для произвольного подмножества $\mathfrak{F}$ комплексной плоскости $\mathbb{C}$.

Остановимся для определённости на пространстве OL(F) (пространство CL(F) может быть рассмотрено аналогично). Скажем, что произвольная функция $f: \mathfrak{F} \rightarrow \mathbb{C}$ принадлежит пространству $\mathrm{OL}(\mathfrak{F})$, если существует константа $C \geqslant 0$ такая, что неравенство (3.1.3) имеет место для всех нормальных операторов $N_{1}$ и $N_{2}$ с конечными спектрами, лежащими в $\mathfrak{F}$. Отметим, что конечность спектров позволяет определить $f\left(N_{1}\right)$ и $f\left(N_{2}\right)$ для произвольной функции $f$. Ясно, что $\mathrm{OL}(\mathfrak{F}) \subset \operatorname{Lip}(\mathfrak{F})$. Таким образом, каждая функция $f$ допускает липшицево продолжение на замыкание $\operatorname{clos} \mathfrak{F}$ множества $\mathfrak{F}$. Из равенства (3.1.9) видно, что эта продолженная функция попадёт в пространство OL(clos $\mathfrak{F})$ и её OL-полунорма не изменится. Таким образом, пространство OL(F) отождествляется естественным образом с пространством OL(clos $\mathfrak{F})$.

Принимая это во внимание, мы будем для краткости использовать обозначения $\mathrm{OL}(\mathbb{D}), \mathrm{OL}\left(\mathbb{C}_{+}\right), \mathrm{CL}(\mathbb{D})$ и $\mathrm{CL}\left(\mathbb{C}_{+}\right)$вместо $\mathrm{OL}(\operatorname{clos} \mathbb{D}), \mathrm{OL}\left(\operatorname{clos} \mathbb{C}_{+}\right), \mathrm{CL}(\operatorname{clos} \mathbb{D})$ и $\mathrm{CL}\left(\operatorname{clos} \mathbb{C}_{+}\right)$.

\section{2. Ограниченные и неограниченные нормальные операторы}

Мы докажем в этом разделе некоторые вспомогательные утверждения, из которых вытекает, что в определении операторно липшицевых и коммутаторно липшицевых (равно как и операторно гёльдеровых) функций можно рассматривать лишь ограниченные нормальные операторы или допустить к рассмотрению неограниченные. В обоих случаях мы получим те же самые классы функций с теми же нормами.

Пусть $N_{1}$ и $N_{2}$ - не обязательно ограниченные нормальные операторы в гильбертовых пространствах $\mathscr{H}_{1}$ и $\mathscr{H}_{2}$ с областями определения $\mathcal{D}_{N_{1}}$ и $\mathcal{D}_{N_{2}}$. Пусть $R$ - ограниченный оператор из $\mathscr{H}_{2}$ в $\mathscr{H}_{1}$. Мы говорим, что $N_{1} R-R N_{2}-$ ограниченный оператор, если $R\left(\mathcal{D}_{N_{2}}\right) \subset \mathcal{D}_{N_{1}}$ и $\left\|N_{1} R u-R N_{2} u\right\| \leqslant C\|u\|$ для 
всех $u \in \mathcal{D}_{N_{2}}$. Тогда существует единственный ограниченный оператор $K$ такой, что $K u=N_{1} R u-R N_{2} u$ для всех $u \in \mathscr{D}_{N_{2}}$. В этом случае мы пишем $K=N_{1} R-R N_{2}$. Таким образом, $N_{1} R-R N_{2}$ - ограниченный оператор в том и только том случае, когда

$$
\left|\left(R u, N_{1}^{*} v\right)-\left(N_{2} u, R^{*} v\right)\right| \leqslant C\|u\| \cdot\|v\|
$$

для всех $u \in \mathcal{D}_{N_{2}}$ и $v \in \mathcal{D}_{N_{1}^{*}}=\mathcal{D}_{N_{1}}$. Легко видеть, что $N_{1} R-R N_{2}$ - ограниченный оператор в том и только том случае, если $N_{2}^{*} R^{*}-R^{*} N_{1}^{*}$ - ограниченный оператор. При этом $\left(N_{1} R-R N_{2}\right)^{*}=-\left(N_{2}^{*} R^{*}-R^{*} N_{1}^{*}\right)$. В частности, мы пишем $N_{1} R=R N_{2}$, если $R\left(\mathcal{D}_{N_{2}}\right) \subset \mathcal{D}_{N_{1}}$ и $N_{1} R u=R N_{2} u$ для всех $u \in \mathcal{D}_{N_{2}}$. Будем говорить, что $\left\|N_{1} R-R N_{2}\right\|=\infty$, если $N_{1} R-R N_{2}$ не является ограниченным оператором.

ЗАмечАниЕ. Пусть $N_{1}$ и $N_{2}$ - нормальные операторы. Предположим, что оператор $N_{1}^{*}$ является замыканием оператора $N_{1 \mathrm{~b}}$, а оператор $N_{2}$ - замыканием оператора $N_{2 \sharp}$. Тогда, если неравенство (3.2.1) выполняется для всех $u \in \mathcal{D}_{N_{2 \sharp}}$ и $v \in \mathcal{D}_{N_{1 b}}$, то оно будет выполняться для всех $u \in \mathcal{D}_{N_{2}}$ и $v \in \mathcal{D}_{N_{1}}$.

Tеорема 3.2.1. Пусть $N_{1}$ и $N_{2}$ - нормальные операторь в гильбертовых пространствах $\mathscr{H}_{1}$ и $\mathscr{H}_{2}$, а $R$ - ограниченный оператор из $\mathscr{H}_{2}$ в $\mathscr{H}_{1}$. Тогда существуют последовательности $\left\{N_{1, n}\right\}_{n \geqslant 1} u\left\{N_{2, n}\right\}_{n \geqslant 1}$ ограниченных нормалъных операторов, действующих в гильбертовых пространствах $\mathscr{H}_{1, n}$ u $\mathscr{H}_{2, n}$, и последовательность $\left\{R_{n}\right\}_{n \geqslant 1}$ ограниченных операторов из $\mathscr{H}_{2, n}$ в $\mathscr{H}_{1, n}$ maкuе, что

(a) последовательность $\left\{\left\|R_{n}\right\|\right\}_{n \geqslant 1}$ не убывает $u \lim _{n \rightarrow \infty}\left\|R_{n}\right\|=\|R\|$;

(b) $\sigma\left(N_{1, n}\right) \subset \sigma\left(N_{1}\right)$ u $\sigma\left(N_{2, n}\right) \subset \sigma\left(N_{2}\right)$ nри всех $n \geqslant 1$;

(c) для любой непрерьвной на множестве $\sigma\left(N_{1}\right) \cup \sigma\left(N_{2}\right)$ функции $f$ последовательность $\left\{\left\|f\left(N_{1, n}\right) R_{n}-R_{n} f\left(N_{2, n}\right)\right\|\right\}_{n \geqslant 1}$ не убъвает и

$$
\lim _{n \rightarrow \infty}\left\|f\left(N_{1, n}\right) R_{n}-R_{n} f\left(N_{2, n}\right)\right\|=\left\|f\left(N_{1}\right) R-R f\left(N_{2}\right)\right\|
$$

(d) для любой непрерьвной на множестве $\sigma\left(N_{1}\right) \cup \sigma\left(N_{2}\right)$ функции $f$ такой, что $\left\|f\left(N_{1}\right) R-R f\left(N_{2}\right)\right\|<\infty$, и любого натурального числа $j$ последовательность сингулярных чисел $\left\{s_{j}\left(f\left(N_{1, n}\right) R_{n}-R_{n} f\left(N_{2, n}\right)\right)\right\}_{n \geqslant 0}$ не убывает и

$$
\lim _{n \rightarrow \infty} s_{j}\left(f\left(N_{1, n}\right) R_{n}-R_{n} f\left(N_{2, n}\right)\right)=s_{j}\left(f\left(N_{1}\right) R-R f\left(N_{2}\right)\right) .
$$

ДокАзАтЕльство. Не умаляя общности, можно считать, что $0 \in \sigma\left(N_{1}\right) \cup$ $\sigma\left(N_{2}\right)$. Положим $P_{1, n} \stackrel{\text { def }}{=} E_{N_{1}}(\{|\lambda| \leqslant n\})$ и $P_{2, n} \stackrel{\text { def }}{=} E_{N_{2}}(\{|\lambda| \leqslant n\})$, где $E_{N_{1}}$ и $E_{N_{2}}$ - спектральные меры нормальных операторов $N_{1}$ и $N_{2}$. Положим

$$
\begin{aligned}
& \widetilde{N}_{1, n} \stackrel{\text { def }}{=} P_{1, n} N_{1}=N_{1} P_{1, n}=P_{1, n} N_{1} P_{1, n}, \\
& \widetilde{N}_{2, n} \stackrel{\text { def }}{=} P_{2, n} N_{2}=N_{2} P_{2, n}=P_{2, n} N_{2} P_{2, n}, \\
& \mathscr{H}_{1, n} \stackrel{\text { def }}{=} P_{1, n} \mathscr{H}_{1} \quad \text { и } \quad \mathscr{H}_{2, n} \stackrel{\text { def }}{=} P_{2, n} \mathscr{H}_{2} .
\end{aligned}
$$

Ясно, что $\widetilde{N}_{1, n}$ и $\widetilde{N}_{2, n}$ - ограниченные нормальные операторы в гильбертовых пространствах $\mathscr{H}_{1}$ и $\mathscr{H}_{2}$, а $\mathscr{H}_{1, n}$ и $\mathscr{H}_{2, n}$ - приводящие подпространства операторов $\widetilde{N}_{1, n}$ и $\widetilde{N}_{2, n}$ соответственно. 
Положим $N_{1, n} \stackrel{\text { def }}{=} \widetilde{N}_{1, n} \mid \mathscr{H}_{1, n}$ и $N_{2, n} \stackrel{\text { def }}{=} \widetilde{N}_{2, n} \mid \mathscr{H}_{2, n}$. Тогда $N_{1, n}$ и $N_{2, n}$ - нормальные операторы в $\mathscr{H}_{1, n}$ и $\mathscr{H}_{2, n}$. Оператор $R_{n}$ из $\mathcal{B}\left(\mathscr{H}_{2, n}, \mathscr{H}_{1, n}\right)$ определяется равенством $R_{n} u \stackrel{\text { def }}{=} P_{1, n} R u=P_{1, n} R P_{2, n} u$ для $u \in \mathscr{H}_{2, n}$. Утверждения (а) и (b) очевидны. Чтобы доказать остальные утверждения, достаточно заметить, что

$$
P_{1, n}\left(f\left(N_{1}\right) R-R f\left(N_{2}\right)\right) P_{2, n} u=\left(f\left(N_{1, n}\right) R_{n}-R_{n} f\left(N_{2, n}\right)\right) u
$$

для всех $u$ из $\mathscr{H}_{2, n}$. Теорема доказана.

\section{3. Разделённые разности и коммутаторная липшицевость}

$\mathrm{C}$ каждой функцией $f$, заданной на замкнутом множестве $\mathfrak{F} \subset \mathbb{C}$, мы связываем функцию $\mathfrak{D}_{0} f: \mathfrak{F} \times \mathfrak{F} \rightarrow \mathbb{C}$,

$$
\left(\mathfrak{D}_{0} f\right)(z, w) \stackrel{\text { def }}{=} \begin{cases}\frac{f(z)-f(w)}{z-w}, & \text { если } z \neq w \\ 0, & \text { если } z=w .\end{cases}
$$

Если множество $\mathfrak{F}$ не имеет изолированных точек и в каждой точке $z$ множества $\mathfrak{F}$ существует конечная производная $f^{\prime}(z)$ в комплексном смысле, то мы можем определить разделённую разность $\mathfrak{D} f: \mathfrak{F} \times \mathfrak{F} \rightarrow \mathbb{C}$ равенством

$$
(\mathfrak{D} f)(z, w) \stackrel{\text { def }}{=} \begin{cases}\frac{f(z)-f(w)}{z-w}, & \text { если } z \neq w, \\ f^{\prime}(z), & \text { если } z=w .\end{cases}
$$

TеОрема 3.3.1. Пусть $f$ - непрерьвная функция на оббединении $\mathfrak{F}_{1} \cup \mathfrak{F}_{2}$ замкнутых подмножеств $\mathfrak{F}_{1} \quad$ u $\mathfrak{F}_{2}$ комплексной плоскости $\mathbb{C}$. Тогда $f \in$ $\mathrm{CL}\left(\mathfrak{F}_{1}, \mathfrak{F}_{2}\right)$ в том и только том случае, если $\mathfrak{D}_{0} f \in \mathfrak{M}\left(\mathfrak{F}_{1} \times \mathfrak{F}_{2}\right)$. При этом

$$
\|f\|_{\mathrm{CL}\left(\mathfrak{F}_{1}, \mathfrak{F}_{2}\right)}=\left\|\mathfrak{D}_{0} f\right\|_{\mathfrak{M}_{0}\left(\mathfrak{F}_{1} \times \mathfrak{F}_{2}\right)} \leqslant\left\|\mathfrak{D}_{0} f\right\|_{\mathfrak{M}_{\left(\mathfrak{F}_{1} \times \mathfrak{F}_{2}\right)} \leqslant 2\|f\|_{\mathrm{CL}\left(\mathfrak{F}_{1}, \mathfrak{F}_{2}\right)} .}
$$

ДокАЗАТЕЛьство. Ограничимся доказательством равенства $\|f\|_{\mathrm{CL}\left(\mathfrak{F}_{1}, \mathfrak{F}_{2}\right)}=$ $\left\|\mathfrak{D}_{0} f\right\|_{\mathfrak{M}_{0}\left(\mathfrak{F}_{1} \times \mathfrak{F}_{2}\right)}$, поскольку всё остальное будет тогда следовать из следствия 2.1.2. Рассмотрим сначала случай конечных множеств $\mathfrak{F}_{1}$ и $\mathfrak{F}_{2}$. Пусть $N_{1}$ и $N_{2}$ - нормальные операторы такие, что $\sigma\left(N_{1}\right) \subset \mathfrak{F}_{1}$ и $\sigma\left(N_{2}\right) \subset \mathfrak{F}_{2}$. В силу теоремы 3.1.11 можно считать, что операторы $N_{1}$ и $N_{2}$ имеют простой спектр. Тогда в пространствах $\mathscr{H}_{1}$ и $\mathscr{H}_{2}$ существуют ортонормированные базисы $\left\{u_{\lambda}\right\}_{\lambda \in \sigma\left(N_{1}\right)}$ и $\left\{v_{\mu}\right\}_{\mu \in \sigma\left(N_{2}\right)}$ такие, что $N_{1} u_{\lambda}=\lambda u_{\lambda}$ при всех $\lambda \in \sigma\left(N_{1}\right)$ и $N_{2} v_{\mu}=\mu v_{\mu}$ при всех $\mu \in \sigma\left(N_{2}\right)$. С каждым оператором $X: \mathscr{H}_{2} \rightarrow \mathscr{H}_{1}$ мы связываем матрицу $\left\{\left(X v_{\mu}, u_{\lambda}\right)\right\}_{(\lambda, \mu) \in \sigma\left(N_{1}\right) \times \sigma\left(N_{2}\right)}$. Имеем

$$
\left(\left(N_{1} R-R N_{2}\right) v_{\mu}, u_{\lambda}\right)=\left(R v_{\mu}, N_{1}^{*} u_{\lambda}\right)-\left(R N_{2} v_{\mu}, u_{\lambda}\right)=(\lambda-\mu)\left(R v_{\mu}, u_{\lambda}\right) .
$$

Аналогично, $\left(\left(f\left(N_{1}\right) R-R f\left(N_{2}\right)\right) v_{\mu}, u_{\lambda}\right)=(f(\lambda)-f(\mu))\left(R v_{\mu}, u_{\lambda}\right)$. Ясно, что

$$
\left\{(f(\lambda)-f(\mu))\left(R v_{\mu}, u_{\lambda}\right)\right\}=\left\{\left(\mathfrak{D}_{0} f\right)(\lambda, \mu)\right\} \star\left\{(\lambda-\mu)\left(R v_{\mu}, u_{\lambda}\right)\right\} .
$$

Заметим, что матрица $\left\{a_{\lambda \mu}\right\}_{(\lambda, \mu) \in \sigma\left(N_{1}\right) \times \sigma\left(N_{2}\right)}$ представима в виде

$$
\left\{a_{\lambda \mu}\right\}_{(\lambda, \mu) \in \sigma\left(N_{1}\right) \times \sigma\left(N_{2}\right)}=\left\{(\lambda-\mu)\left(R v_{\mu}, u_{\lambda}\right)\right\}_{(\lambda, \mu) \in \sigma\left(N_{1}\right) \times \sigma\left(N_{2}\right)},
$$


где $R$ - оператор, действующий из $\mathscr{H}_{2}$ в $\mathscr{H}_{1}$, в том и только том случае, когда $a_{\lambda \mu}=0$ при $\lambda=\mu$. Теперь равенство $\|f\|_{\mathrm{CL}\left(\mathfrak{F}_{1}, \mathfrak{F}_{2}\right)}=\left\|\mathfrak{D}_{0} f\right\|_{\mathfrak{M}_{0}\left(\mathfrak{F}_{1} \times \mathfrak{F}_{2}\right)}$ в случае конечных множеств $\mathfrak{F}_{1}$ и $\mathfrak{F}_{2}$ очевидно.

Сведём теперь общий случай к разобранному случаю конечных множеств $\mathfrak{F}_{1}$ и $\mathfrak{F}_{2}$.

Неравенство $\|f\|_{\mathrm{CL}\left(\mathfrak{F}_{1}, \mathfrak{F}_{2}\right)} \geqslant\left\|\mathfrak{D}_{0} f\right\|_{\mathfrak{M}_{0}\left(\mathfrak{F}_{1} \times \mathfrak{F}_{2}\right)}$ очевидным образом сводится к случаю конечных множеств $\mathfrak{F}_{1}$ и $\mathfrak{F}_{2}$.

Займёмся теперь неравенством $\|f\|_{\mathrm{CL}\left(\mathfrak{F}_{1}, \mathfrak{F}_{2}\right)} \leqslant\left\|\mathfrak{D}_{0} f\right\|_{\mathfrak{M}_{0}\left(\mathfrak{F}_{1} \times \mathfrak{F}_{2}\right)}$, которое означает, что $\left\|f\left(N_{1}\right) R-R f\left(N_{2}\right)\right\| \leqslant\left\|\mathfrak{D}_{0} f\right\|_{\mathfrak{M}_{0}\left(\mathfrak{F}_{1} \times \mathfrak{F}_{2}\right)}\left\|N_{1} R-R N_{2}\right\|$ для любого ограниченного оператора $R$ и для любых ограниченных нормальных операторов $N_{1}$ и $N_{2}$ таких, что $\sigma\left(N_{1}\right) \subset \mathfrak{F}_{1}$ и $\sigma\left(N_{2}\right) \subset \mathfrak{F}_{1}$. Из разобранного выше частного случая следует, что это неравенство заведомо выполняется с случае, когда нормальные операторы $N_{1}$ и $N_{2}$ имеют конечные спектры. Случай произвольных нормальных операторов $N_{1}$ и $N_{2}$ со спектрами в $\mathfrak{F}_{1}$ и $\mathfrak{F}_{2}$ соответственно сводится к этому частному случаю при помощи леммы 3.1.12. Теорема доказана.

ЗАмЕчаниЕ. Неравенство $\|f\|_{\mathrm{CL}\left(\mathfrak{F}_{1}, \mathfrak{F}_{2}\right)} \leqslant\left\|\mathfrak{D}_{0} f\right\|_{\mathfrak{M}\left(\mathfrak{F}_{1} \times \mathfrak{F}_{2}\right)}$ можно доказать и с помощью двойных операторных интегралов (см. замечание к теореме 3.5.2).

Теорема 3.3 .1 в случае, когда $\mathfrak{F}_{1}=\mathfrak{F}_{2}$, приводит к следующему результату.

ТеОРема 3.3.2. Пусть $f$ - функиия, заданная на непустом замкнутом подмножестве $\mathfrak{F}$ комплексной плоскости $\mathbb{C}$. Тогда $f \in \mathrm{CL}(\mathfrak{F})$ в том и только том случае, когда $\mathfrak{D}_{0} f \in \mathfrak{M}(\mathfrak{F} \times \mathfrak{F})$. При этом

$$
\|f\|_{\mathrm{CL}(\mathfrak{F})}=\left\|\mathfrak{D}_{0} f\right\|_{\mathfrak{M}_{0}(\mathfrak{F} \times \mathfrak{F})} \leqslant\left\|\mathfrak{D}_{0} f\right\|_{\mathfrak{M}(\mathfrak{F} \times \mathfrak{F})} \leqslant 2\|f\|_{\mathrm{CL}(\mathfrak{F})} .
$$

Заметим, что если $\mathfrak{D}_{0} f \in \mathfrak{M}(\mathfrak{F} \times \mathfrak{F})$ для функции $f$, заданной на $\mathscr{F}$, то $f$ непрерывна и даже удовлетворяет условию Липшица. Действительно, если $\zeta, \tau \in \mathfrak{F}$, то $\left|\left(\mathfrak{D}_{0} f\right)(\tau, \zeta)\right| \leqslant\left\|\mathfrak{D}_{0} f\right\|_{\mathfrak{M}_{0}(\mathfrak{F} \times \mathfrak{F})}$, откуда $|f(\zeta)-f(\tau)| \leqslant\left\|\mathfrak{D}_{0} f\right\|_{\mathfrak{M}_{0}(\mathfrak{F} \times \mathfrak{F})}|\zeta-\tau|$.

Следующее утверждение получено в работе [32].

ТЕОРема 3.3.3. Пусть $f$ - функиия на замкнутом подмножестве $\mathfrak{F}$ комплексной плоскости $\mathbb{C}$ такая, что $\mathfrak{D}_{0} f \in \mathfrak{M}(\mathfrak{F} \times \mathfrak{F})$. Тогда функиия $f$ дифферениируема в комплексном смысле в каждой неизолированной точке мно-

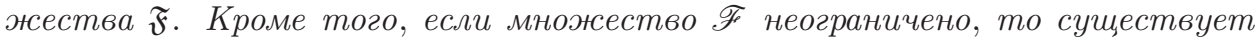
конечный предел $\lim _{|z| \rightarrow \infty} z^{-1} f(z)$.

Нам понадобится элементарная лемма, которую мы приводим без доказательства.

Лемма 3.3.4. Пусть $S$ и $T$ - произвольные множества. Предположим, что последователъность функиий $\left\{\boldsymbol{\varphi}_{n}\right\}$ на $S \times T$ сходится поточечно $к$ функиии ч. Тогда $\|\varphi\|_{\mathfrak{M}(S \times T)} \leqslant \underline{\lim }_{n \rightarrow \infty}\left\|\boldsymbol{\varphi}_{n}\right\|_{\mathfrak{M}(S \times T)}$.

ДокАЗАТЕЛЬСтво теоремы 3.3.3. Докажем сначала дифференцируемость в каждой неизолированной точке $a$ множества $\mathfrak{F}$. Не умаляя общности, можно считать, что $a=0$ и $f(0)=0$. Нужно доказать, что функция $z^{-1} f(z)$ имеет конечный предел при $z \rightarrow 0$. Предположим, что эта функция имеет по крайней мере два конечных (поскольку функция $f$ липшицева) предельных значения при $z \rightarrow 0$. Ясно, что можно считать этими предельными значениями числа 1 
и -1. Таким образом, существуют две стремящиеся к нулю последовательности $\left\{\lambda_{n}\right\}_{n \geqslant 1}$ и $\left\{\mu_{n}\right\}_{n \geqslant 1}$ точек множества $\mathfrak{F} \backslash\{0\}$ такие, что $\lim _{n \rightarrow \infty} \lambda_{n}^{-1} f\left(\lambda_{n}\right)=1$ и $\lim _{n \rightarrow \infty} \mu_{n}^{-1} f\left(\mu_{n}\right)=-1$. Переходя, если нужно, к подпоследовательностям, мы можем добиться выполнения следующих условий:

(a) $\left|\lambda_{n}\right|>\left|\mu_{n}\right|>\left|\lambda_{n+1}\right|$ при всех $n \geqslant 1$;

(b) $\lim _{n \rightarrow \infty} \mu_{n}^{-1} \lambda_{n}=0$ и $\lim _{n \rightarrow \infty} \lambda_{n+1}^{-1} \mu_{n}=0$.

Ясно, что $\left\|\left\{\left(\mathfrak{D}_{0} f\right)\left(\lambda_{m}, \mu_{n}\right)\right\}\right\|_{\mathfrak{M}(\mathbb{N} \times \mathbb{N})} \leqslant\left\|\mathfrak{D}_{0} f\right\|_{\mathfrak{M}(\mathfrak{F} \times \mathfrak{F})}$. Заметим, что последовательность $\left\{\left\|\left\{\left(\mathfrak{D}_{0} f\right)\left(\lambda_{m+k}, \mu_{n+k}\right)\right\}\right\|_{\mathfrak{M}(\mathbb{N} \times \mathbb{N})}\right\}_{k \geqslant 1}$ является невозрастающей и

$$
\lim _{k \rightarrow \infty}\left(\mathfrak{D}_{0} f\right)\left(\lambda_{m+k}, \mu_{n+k}\right)=\operatorname{sgn}\left(m-n+\frac{1}{2}\right) .
$$

Теперь из леммы 3.3 .4 следует, что $\|\{\operatorname{sgn}(m-n+1 / 2)\}\|_{\mathfrak{M}(\mathbb{N} \times \mathbb{N})}<+\infty$, что противоречит теореме 2.2.7.

Существование конечного предела $\lim _{|z| \rightarrow \infty} z^{-1} f(z)$ в случае неограниченного множества $\mathfrak{F}$ доказывается аналогичным образом с той лишь разницей, что теперь нужно выбирать последовательности $\left\{\lambda_{n}\right\}_{n \geqslant 1}$ и $\left\{\mu_{n}\right\}_{n \geqslant 1}$, стремящиеся к бесконечности. Теорема доказана.

СлЕДСТвИЕ 3.3.5. Пространство $\mathrm{CL}(\mathbb{C})$ совпадает с множеством всех функций вида $a z+b$, где $a, b \in \mathbb{C}$.

ДокАЗАТЕЛЬСтво. Ясно, что любая функция вида $a z+b$, где $a, b \in \mathbb{C}$, принадлежит пространству $\mathrm{CL}(\mathbb{C})$. Обратно, из теоремы 3.3 .3 следует, что $f$ - целая функция. Ясно, что функция $f^{\prime}$ ограничена, поскольку $\mathrm{CL}(\mathbb{C}) \subset \mathrm{OL}(\mathbb{C}) \subset$ $\operatorname{Lip}(\mathbb{C})$. Остаётся воспользоваться теоремой Лиувилля.

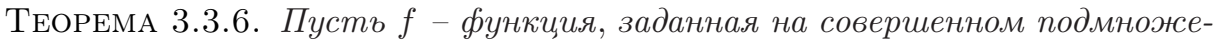
стве $\mathfrak{F}$ комплексной плоскости $\mathbb{C}$. Тогда $f \in \mathrm{CL}(\mathfrak{F})$ в том и только том случае, когда $f$ дифференцируема в комплексном смысле в каждой точке множества $\mathfrak{F}$ и $\mathfrak{D} f \in \mathfrak{M}(\mathfrak{F} \times \mathfrak{F})$. При этом $\|f\|_{\mathrm{CL}(\mathfrak{F})}=\|\mathfrak{D} f\|_{\mathfrak{M}(\mathfrak{F} \times \mathfrak{F})}$.

ДокАзАтельство. Если $f \in \mathrm{CL}(\mathfrak{F})$, то $\mathfrak{D}_{0} f \in \mathfrak{M}(\mathfrak{F} \times \mathfrak{F})$ в силу теоремы 3.3 .2 и следствия 2.1.2. Дифференцируемость функции $f$ следует из теоремы 3.3.3. Обратно, если $\mathfrak{D} f \in \mathfrak{M}(\mathfrak{F} \times \mathfrak{F})$, то $\mathfrak{D}_{0} f \in \mathfrak{M}_{0}(\mathfrak{F} \times \mathfrak{F})$ и можно применить теорему 3.3.2. Равенство $\|f\|_{\mathrm{CL}(\mathfrak{F})}=\|\mathfrak{D} f\|_{\mathfrak{M}(\mathfrak{F} \times \mathfrak{F})}$ следует из теоремы 3.3.2, леммы 2.1.3 и из очевидного равенства $\|\mathfrak{D} f\|_{\mathfrak{M}_{0}(\mathfrak{F} \times \mathfrak{F})}=\left\|\mathfrak{D}_{0} f\right\|_{\mathfrak{M}_{0}(\mathfrak{F} \times \mathfrak{F})}$. Теорема доказана.

Следующая теорема показывает, что для оценки квазикоммутаторной нормы нет необходимости рассматривать все нормальные операторы $N_{1}$ и $N_{2}$, а достаточно ограничиться только одной парой нормальных операторов $N_{1}$ и $N_{2}$ таких, что $\sigma\left(N_{1}\right)=\mathfrak{F}_{1}$ и $\sigma\left(N_{2}\right)=\mathfrak{F}_{2}$. В частности, когда речь идёт о пространстве $\mathrm{CL}(\mathfrak{F})$, можно считать, что $N_{1}=N_{2}$, т. е. ограничиться одним нормальным оператором $N=N_{1}=N_{2}$ таким, что $\sigma(N)=\mathfrak{F}$.

Теорема 3.3.7. Пусть $N_{1}$ и $N_{2}$ - нормальные операторы в гилъбертовых пространствах $\mathscr{H}_{1}$ и $\mathscr{H}_{2}$. Предположим, что непрерывная функиия $f$ на $\sigma\left(N_{1}\right) \cup \sigma\left(N_{2}\right)$ обладает следующим свойством:

$$
\left\|f\left(N_{1}\right) R-R f\left(N_{2}\right)\right\| \leqslant\left\|N_{1} R-R N_{2}\right\|
$$

для всех $R \in \mathcal{B}\left(\mathscr{H}_{2}, \mathscr{H}_{1}\right)$. Тогда $f \in \mathrm{CL}\left(\sigma\left(N_{1}\right), \sigma\left(N_{2}\right)\right) u\|f\|_{\mathrm{CL}\left(\sigma\left(N_{1}\right), \sigma\left(N_{2}\right)\right)} \leqslant 1$. 
Пусть $f$ - непрерывная функция на подмножестве комплексной плоскости. Предположим, что $N_{1}$ и $N_{2}$ - нормальные операторы в гильбертовых пространствах $\mathscr{H}_{1}$ и $\mathscr{H}_{2}$, объединение спектров которых содержится в области определения функции $f$. Будем говорить, что пара $\left(N_{1}, N_{2}\right)$ является $f$-регулярной, если неравенство (3.3.2) справедливо при всех $R \in \mathcal{B}\left(\mathscr{H}_{2}, \mathscr{H}_{1}\right)$.

Теорему 3.3.7 можно переформулировать следующим образом.

Если упорядоченная пара нормальных операторов $\left(N_{1}, N_{2}\right)$ является $f$-регулярной, то $f$-регулярной будет и любая пара нормальных операторов $\left(M_{1}, M_{2}\right)$ такая, что $\sigma\left(M_{1}\right) \subset \sigma\left(N_{1}\right)$ u $\sigma\left(M_{2}\right) \subset \sigma\left(N_{2}\right)$.

Докажем сначала лемму.

Лемма 3.3.8. Пусть $\left(N_{1}, N_{2}\right)$ - $f$-регулярная пара ограниченнъх нормальных операторов в $\mathscr{H}_{1}$ и $\mathscr{H}_{2}$, а $\mathscr{K}_{1}$ u $\mathscr{K}_{2}$ - приводящие подпространства этих операторов. Если оператор $M_{1}$ унитарно эквивалентен оператору $N_{1} \mid \mathscr{K}_{1}$, а оператор $M_{2}$ унитарно эквивалентен оператору $N_{2} \mid \mathscr{K}_{2}$, mо пара $\left(M_{1}, M_{2}\right)$ является $f$-регулярной.

ДоказАтельство. Пусть $M_{1} \in \mathcal{B}\left(\widetilde{\mathscr{H}}_{1}\right)$ и $M_{2} \in \mathcal{B}\left(\widetilde{\mathscr{H}}_{2}\right)$. Достаточно рассмотреть следующие два частных случая.

1. $\mathscr{K}_{1}=\mathscr{H}_{1}$ и $\mathscr{K}_{2}=\mathscr{H}_{2}$. Тогда $M_{1}=U_{1}^{*} N_{1} U_{1}$ и $M_{2}=U_{2}^{*} N_{2} U_{2}$ для некоторых унитарных операторов. Для любого оператора $R$ из $\mathcal{B}\left(\widetilde{\mathscr{H}}_{2}, \widetilde{\mathscr{H}}_{1}\right)$ имеем:

$$
\begin{aligned}
\left\|f\left(M_{1}\right) R-R f\left(M_{2}\right)\right\| & =\left\|U_{1}^{*} f\left(N_{1}\right) U_{1} R-R U_{2}^{*} f\left(N_{2}\right) U_{2}\right\| \\
& =\left\|f\left(N_{1}\right) U_{1} R U_{2}^{*}-U_{1} R U_{2}^{*} f\left(N_{2}\right)\right\| \\
& \leqslant\left\|N_{1} U_{1} R U_{2}^{*}-U_{1} R U_{2}^{*} N_{2}\right\|=\left\|M_{1} R-R M_{2}\right\| .
\end{aligned}
$$

2. $M_{1}=N_{1} \mid \mathscr{K}_{1}$ и $M_{2}=N_{2} \mid \mathscr{K}_{2}$. Пусть $P_{1}$ - ортогональный проектор из $\mathscr{H}_{1}$ на $\mathscr{K}_{1}$ и $P_{2}$ - ортогональный проектор из $\mathscr{H}_{2}$ на $\mathscr{K}_{2}$. Если $R \in \mathcal{B}\left(\mathscr{K}_{2}, \mathscr{K}_{1}\right)$, то

$$
\begin{aligned}
\left\|f\left(M_{1}\right) R-R f\left(M_{2}\right)\right\| & =\left\|P_{1}\left(f\left(M_{1}\right) R-R f\left(M_{2}\right)\right) P_{2}\right\| \\
& =\left\|P_{1}\left(f\left(N_{1}\right) R-R f\left(N_{2}\right)\right) P_{2}\right\| \\
& =\left\|f\left(N_{1}\right) P_{1} R P_{2}-P_{1} R P_{2} f\left(N_{2}\right)\right\| \leqslant\left\|N_{1} P_{1} R P_{2}-P_{1} R P_{2} N_{2}\right\| \\
& =\left\|M_{1} R-R M_{2}\right\| .
\end{aligned}
$$

Лемма доказана.

ДоКАЗАТЕЛЬСТВо тЕОРЕмЫ 3.3.7. В силу теоремы 3.2.1 достаточно рассмотреть случай ограниченных операторов $N_{1}$ и $N_{2}$. Теорема 3.3 .7 в случае, когда спектры операторов $N_{1}$ и $N_{2}$ конечны, мгновенно вытекает из леммы 3.3 .8 и теоремы 3.1.11. Из леммы 3.1 .12 следует, что остаётся доказать, что для любых конечных подмножеств $\Delta_{1}$ и $\Delta_{2}$ множеств $\sigma\left(N_{1}\right)$ и $\sigma\left(N_{2}\right)$ найдутся нормальные операторы $M_{1} \in \mathcal{B}\left(\mathscr{K}_{1}\right)$ и $M_{2} \in \mathcal{B}\left(\mathscr{K}_{2}\right)$ такие, что $\sigma\left(M_{1}\right)=\Delta_{1}$, $\sigma\left(M_{2}\right)=\Delta_{2}$ и $\left\|f\left(M_{1}\right) R-R f\left(M_{2}\right)\right\| \leqslant\left\|M_{1} R-R M_{2}\right\|$ для всех $R$ из $\mathcal{B}\left(\mathscr{K}_{2}, \mathscr{K}_{1}\right)$. C каждым нормальным оператором $N$ свяжем функцию $\alpha_{N}$ такую, что $\alpha_{N}(\zeta)$ есть кратность спектра оператора $N$ в изолированной точке $\zeta$ его спектра $\sigma(N)$ и $\alpha_{N}(\zeta)=\infty$ в каждой неизолированной точке его спектра. 
В качестве операторов $M_{1}$ и $M_{2}$ мы возьмём какие-либо нормальные операторы в гильбертовых пространствах $\mathscr{K}_{1}$ и $\mathscr{K}_{2}$, обладающие следующими свойствами:

1) $\sigma\left(M_{1}\right)=\Delta_{1}$ и $\sigma\left(M_{2}\right)=\Delta_{2}$;

2) функции $\alpha_{M_{1}}$ и $\alpha_{M_{2}}$ являются сужениями функций $\alpha_{N_{1}}$ и $\alpha_{N_{2}}$.

Пусть $\Delta_{1}^{(\varepsilon)}$ и $\Delta_{2}^{(\varepsilon)}$ обозначают замкнутые $\varepsilon$-окрестности множеств $\Delta_{1}$ и $\Delta_{2}$. Пусть $N_{1}^{(\varepsilon)}$ обозначает сужение оператора $N_{1}$ на подпространство $E_{N_{1}}\left(\Delta_{1}^{(\varepsilon)} \cap\right.$ $\left.\sigma\left(N_{1}\right)\right)$, а $N_{2}^{(\varepsilon)}$ - сужение оператора $N_{2}$ на подпространство $E_{N_{2}}\left(\Delta_{2}^{(\varepsilon)} \cap \sigma\left(N_{2}\right)\right)$, где $E_{N_{1}}$ и $E_{N_{2}}$ - спектральные меры операторов $N_{1}$ и $N_{2}$. Ясно, что существуют операторы $M_{1}^{(\varepsilon)}$ из $\mathcal{B}\left(\mathscr{K}_{1}\right)$ и $M_{2}^{(\varepsilon)}$ из $\mathcal{B}\left(\mathscr{K}_{2}\right)$ такие, что оператор $M_{1}^{(\varepsilon)}$ унитарно эквивалентен оператору $N_{1}^{(\varepsilon)}$, оператор $M_{2}^{(\varepsilon)}$ унитарно эквивалентен оператоpy $N_{2}^{(\varepsilon)},\left\|M_{1}-M_{1}^{(\varepsilon)}\right\| \leqslant \varepsilon$ и $\left\|M_{2}-M_{2}^{(\varepsilon)}\right\| \leqslant \varepsilon$. Тогда для любого оператора $R$ из $\mathcal{B}\left(\mathscr{K}_{2}, \mathscr{K}_{1}\right)$ имеем

$$
\begin{gathered}
\left\|f\left(M_{1}\right) R-R f\left(M_{2}\right)\right\| \leqslant\|R\| \cdot\left\|f\left(M_{1}\right)-f\left(M_{1}^{(\varepsilon)}\right)\right\|+\|R\| \cdot\left\|f\left(M_{2}\right)-f\left(M_{2}^{(\varepsilon)}\right)\right\| \\
+\left\|f\left(M_{1}^{(\varepsilon)}\right) R-R f\left(M_{2}^{(\varepsilon)}\right)\right\| \\
\leqslant\|R\| \cdot\left\|f\left(M_{1}\right)-f\left(M_{1}^{(\varepsilon)}\right)\right\|+\|R\| \cdot\left\|f\left(M_{2}\right)-f\left(M_{2}^{(\varepsilon)}\right)\right\| \\
+\left\|M_{1}^{(\varepsilon)} R-R M_{2}^{(\varepsilon)}\right\| \\
\leqslant\|R\| \cdot\left\|f\left(M_{1}\right)-f\left(M_{1}^{(\varepsilon)}\right)\right\|+\|R\| \cdot\left\|f\left(M_{2}\right)-f\left(M_{2}^{(\varepsilon)}\right)\right\| \\
+2 \varepsilon\|R\|+\left\|M_{1} R-R M_{2}\right\| .
\end{gathered}
$$

Остаётся перейти к пределу при $\varepsilon \rightarrow 0$. Теорема доказана.

Следующая теорема содержится в работе [32].

Теорема 3.3.9. Пусть $M$ и $N$ - операторы в гилъбертовом пространстве $\mathscr{H}$, причём оператор $N$ нормальный. Тогда следующие условия равносильны:

(a) $M=f(N)$ для некоторой функиии $f$ из $\mathrm{CL}(\sigma(N))$;

(b) существует константа с такая, что $\|M R-R M\| \leqslant c\|N R-R N\| \partial л я$ всех ограниченных операторов $R$;

(с) существует константа с такая, что $\|M R-R M\|_{S_{1}} \leqslant c\|N R-R N\|_{S_{1}}$ для всех ограниченных операторов $R$;

(d) для любого ограниченного оператора $T$ найдётся ограниченный операmор $S$ такой, что $S N-N S=T M-M T$;

(е) для любого компактного оператора $T$ найдётся ограниченный операmор $S$ mакой, что $S N-N S=T M-M T$;

(f) для любого оператора $T$ из $\boldsymbol{S}_{1}(\mathscr{H})$ найдётся оператор $S$ из $\boldsymbol{S}_{1}(\mathscr{H})$ maкой, что $S N-N S=T M-M T$.

\section{4. Мультипликаторы Шура и операторная липшицевость}

Заметим, что если замкнутое подмножество $\mathfrak{F}$ является множеством Фугледе, то $\mathrm{OL}(\mathfrak{F})=\mathrm{CL}(\mathfrak{F})$ в силу теоремы 3.1.8. Таким образом, в этом случае теорема 3.3.2 даёт полное описание пространства $\mathrm{OL}(\mathfrak{F})$ в терминах мультипликаторов Шура. 
В частности, для множеств $\mathfrak{F}$, лежащих на прямой или окружности, мы имеем полное описание пространства OL(F) в терминах мультипликаторов Шура. Причём в последнем случае и полунорма операторно липшицевой функции выражается через норму мультипликатора Шура.

В случае, когда множество $\mathfrak{F}$ не является множеством Фугледе, нам неизвестно полное описание операторно липшицевых функций на $\mathfrak{F}$ в терминах мультипликаторов Шура.

В этом случае мы можем предложить следующее достаточное условие операторной липшицевости.

ТеОРема 3.4.1. Пусть $f$ - функиия, непрерьвная на замкнутом подмножестве $\mathfrak{F}$ комплексной плоскости $\mathbb{C}$. Предположим, что существуют мультипликаторы Шура $\Phi_{1}, \Phi_{2} \in \mathfrak{M}(\mathfrak{F} \times \mathfrak{F})$ такие, что

$$
f(z)-f(w)=(z-w) \Phi_{1}(z, w)+(\bar{z}-\bar{w}) \Phi_{2}(z, w) .
$$

Тогда $f \in \mathrm{OL}(\mathfrak{F}) u\|f\|_{\mathrm{OL}(\mathfrak{F})} \leqslant\left\|\Phi_{1}\right\|_{\mathfrak{M}(\mathfrak{F} \times \mathfrak{F})}+\left\|\Phi_{2}\right\|_{\mathfrak{M}(\mathfrak{F} \times \mathfrak{F})}$.

Эту теорему можно доказать с помощью аппроксимации операторами с конечными спектрами, как это сделано в доказательстве теоремы 3.3.1. Мы опустим это доказательство, приведя взамен доказательство, основанное на двойных операторных интегралах (см. теорему 3.5.5 и замечание к ней).

ЗАмЕчАниЕ. Теорему 3.4.1 иногда удобнее применять, привлекая вещественные переменные $z=x_{1}+\mathrm{i} y_{1}, w=x_{2}+\mathrm{i} y_{2}$. Предположим, что существуют мультипликаторы Шура $F_{1}, F_{2} \in \mathfrak{M}(\mathfrak{F} \times \mathfrak{F})$ такие, что

$$
f(z)-f(w)=\left(x_{1}-x_{2}\right) F_{1}(z, w)+\left(y_{1}-y_{2}\right) F_{2}(z, w) .
$$

Тогда $f \in \mathrm{OL}(\mathfrak{F})$ и

$\|f\|_{\mathrm{OL}(\mathfrak{F})} \leqslant \frac{1}{2}\left\|F_{1}+\mathrm{i} F_{2}\right\|_{\mathfrak{M}(\mathfrak{F} \times \mathfrak{F})}+\frac{1}{2}\left\|F_{1}-\mathrm{i} F_{2}\right\|_{\mathfrak{M}(\mathfrak{F} \times \mathfrak{F})} \leqslant\left\|F_{1}\right\|_{\mathfrak{M}(\mathfrak{F} \times \mathfrak{F})}+\left\|F_{2}\right\|_{\mathfrak{M}(\mathfrak{F} \times \mathfrak{F})}$.

Tеорема 3.4.2. Пусть $f \in \mathrm{OL}(\mathfrak{F})$, где $\mathfrak{F}$ - замкнутое множество в $\mathbb{C}$. Тогда для любой прямой $l$ сужение $f \mid l \cap \mathfrak{F}$ дифферениируемо в каждой неизолированной точке множества $l \cap \mathfrak{F}$ и в $\infty$, если множество $l \cap \mathfrak{F}$ неограничено.

ДоказАтельство. Ясно, что $f \mid l \cap \mathfrak{F} \in \mathrm{OL}(l \cap \mathfrak{F})$. Остаётся заметить, что $\mathrm{CL}(l \cap \mathfrak{F})=\mathrm{OL}(l \cap \mathfrak{F})$ в силу теоремы 3.1 .10 , и применить теорему 3.3 .6 к функции $f \mid l \cap \mathfrak{F}$.

СлеДСтвиЕ 3.4.3. Пусть $f \in \mathrm{OL}(\mathfrak{F})$, әде $\mathfrak{F}$ - замкнутое подмножество комплексной плоскости. Тогда функиия $f$ дифферениируема по любому направлению в каждой внутренней точке множества $\mathfrak{F}$.

ЗАмЕчаниЕ. Функция $f \in \mathrm{OL}(\mathfrak{F})$ не обязана быть дифференцируемой как функция двух вещественных переменных. Например, нетрудно проверить, что функция $f$, заданная в полярных координатах равенством $f(r, \theta)=r e^{3 i \theta}$, принадлежит пространству $\mathrm{OL}(\mathbb{C})$, но не дифференцируема в нуле как функция двух вещественных переменных $x$ и $y$. Это обстоятельство было отмечено в [13] (см. также [2]). 


\section{5. Роль двойных операторных интегралов}

В этом разделе мы продемонстрируем роль двойных операторных интегралов в оценках операторных разностей и (квази)коммутаторов. Мы начнём с оценок операторных разностей при возмущении самосопряжённого оператора оператором класса Гильберта-Шмидта и обсудим формулу Бирмана-Соломяка.

Далее мы вернёмся к результатам предыдущих двух разделов, где мы получили условия для коммутаторной липшицевости и операторной липшицевости в терминах принадлежности некоторых функций пространству дискретных мультипликаторов Шура. В этом разделе мы приведём другое доказательство достаточности этих условий с помощью двойных операторных интегралов. При этом мы получим полезные формулы, выражающие операторные разности и коммутаторы в терминах двойных операторных интегралов.

Наконец, мы получим формулы для операторных производных в терминах двойных операторных интегралов.

Следующая теорема была получена М. С. Бирманом и М. З. Соломяком в [21].

Теорема 3.5.1. Пусть $f$ - липиицева функиия на $\mathbb{R}$, а $A$ и $B$-самосопряжённые операторы в гильбертовом пространстве, разность $A-B$ которых входит в класс Гилъберта-Шмидта $\boldsymbol{S}_{2}$. Тогда имеет место формула

$$
f(A)-f(B)=\int_{\mathbb{R}} \int_{\mathbb{R}}\left(\mathfrak{D}_{0} f\right)(x, y) d E_{A}(x)(A-B) d E_{B}(y) .
$$

Отметим, что из формулы (3.5.1) непосредственно вытекает неравенство

$$
\|f(A)-f(B)\|_{\boldsymbol{S}_{2}} \leqslant\|f\|_{\text {Lip }}\|A-B\|_{\boldsymbol{S}_{2}} .
$$

Иными словами, липшицевы функции являются $\boldsymbol{S}_{2}$-липшицевыми. Оказывается, что липшицевы функции также являются $\boldsymbol{S}_{p}$-липшицевыми при $p \in(1, \infty)$. Это было недавно доказано в работе [67]. Напомним, что при $p=1$ соответствующее утверждение неверно. Впервые это было доказано в работе [26]. Более того, класс $\boldsymbol{S}_{1}$-липшицевых функций совпадает с классом операторно липшицевых функций (см. теорему 3.6.5).

Займёмся теперь коммутаторной липшицевостью.

ТеОРема 3.5.2. Пусть $\mathfrak{F}_{1} u \mathfrak{F}_{2}$ - замкнутые подмножества плоскости $\mathbb{C}$. Предположим, что $f$ - непрерывная функиия на $\mathfrak{F}_{1} \cup \mathfrak{F}_{2}$ такая, что функиия $\mathfrak{D}_{0} f$, определённая равенством (3.3.1), принадлежит классу мультипликаторов Шура $\mathfrak{M}\left(\mathfrak{F}_{1} \times \mathfrak{F}_{2}\right)$. Тогда если $N_{1}$ и $N_{2}$ - нормальные операторы такие, что $\sigma\left(N_{j}\right) \subset \mathfrak{F}_{j}, j=1,2$, а $R$ - ограниченный линейный оператор, то справедлива формула

$$
f\left(N_{1}\right) R-R f\left(N_{2}\right)=\int_{\mathfrak{F}_{1}} \int_{\mathfrak{F}_{2}}\left(\mathfrak{D}_{0} f\right)\left(\zeta_{1}, \zeta_{2}\right) d E_{1}\left(\zeta_{1}\right)\left(N_{1} R-R N_{2}\right) d E_{2}\left(\zeta_{2}\right),
$$

где $E_{j}$ - спектральная мера оператора $N_{j}$.

ЗАмечАниЕ. Из формулы (3.5.2) сразу же вытекает, что

$$
\begin{aligned}
\left\|f\left(N_{1}\right) R-R f\left(N_{2}\right)\right\| & \leqslant\left\|\mathfrak{D}_{0} f\right\|_{\mathfrak{M}\left(E_{1}, E_{2}\right)}\left\|N_{1} R-R N_{2}\right\| \\
& \leqslant\left\|\mathfrak{D}_{0} f\right\|_{\mathfrak{M}\left(\mathfrak{F}_{1} \times \mathfrak{F}_{2}\right)}\left\|N_{1} R-R N_{2}\right\|
\end{aligned}
$$

и, в частности, $f$ - коммутаторно липшицева функция. 
В частном случае, когда $R$ - единичный оператор, получаем следующий результат.

ТеОРема 3.5.3. Пусть $\mathfrak{F}$ - замкнутое подмножество плоскости $\mathbb{C}$, a $f-$ непрерывная функиия на $\mathfrak{F}$ такая, что $\mathfrak{D}_{0} f \in \mathfrak{M}(\mathfrak{F} \times \mathfrak{F})$. Тогда, если $N_{1}$ и $N_{2}-$ нормальные операторы со спектрами в $\mathfrak{F}$, то справедлива формула

$$
f\left(N_{1}\right)-f\left(N_{2}\right)=\int_{\mathfrak{F}} \int_{\mathfrak{F}}\left(\mathfrak{D}_{0} f\right)\left(\zeta_{1}, \zeta_{2}\right) d E_{1}\left(\zeta_{1}\right)\left(N_{1}-N_{2}\right) d E_{2}\left(\zeta_{2}\right) .
$$

ДоКАЗАТЕЛЬСТВо ТЕОРЕмЫ 3.5.2. Предположим сначала, что операторы $N_{1}$ и $N_{2}$ ограничены. Имеем

$$
\begin{aligned}
\int_{\mathfrak{F}_{1}} & \int_{\mathfrak{F}_{2}}\left(\mathfrak{D}_{0} f\right)\left(\zeta_{1}, \zeta_{2}\right) d E_{1}\left(\zeta_{1}\right)\left(N_{1} R-R N_{2}\right) d E_{2}\left(\zeta_{2}\right) \\
= & \int_{\mathfrak{F}_{1}} \int_{\mathfrak{F}_{2}}\left(\mathfrak{D}_{0} f\right)\left(\zeta_{1}, \zeta_{2}\right) d E_{1}\left(\zeta_{1}\right) N_{1} R d E_{2}\left(\zeta_{2}\right) \\
& -\int_{\mathfrak{F}_{1}} \int_{\mathfrak{F}_{2}}\left(\mathfrak{D}_{0} f\right)\left(\zeta_{1}, \zeta_{2}\right) d E_{1}\left(\zeta_{1}\right) R N_{2} d E_{2}\left(\zeta_{2}\right) .
\end{aligned}
$$

Из определения двойных операторных интегралов следует, что

$$
\begin{aligned}
\int_{\mathfrak{F}_{1}} & \int_{\mathfrak{F}_{2}}\left(\mathfrak{D}_{0} f\right)\left(\zeta_{1}, \zeta_{2}\right) d E_{1}\left(\zeta_{1}\right) N_{1} R d E_{2}\left(\zeta_{2}\right) \\
\quad= & \int_{\mathfrak{F}_{1}} \int_{\mathfrak{F}_{2}} \zeta_{1}\left(\mathfrak{D}_{0} f\right)\left(\zeta_{1}, \zeta_{2}\right) d E_{1}\left(\zeta_{1}\right) R d E_{2}\left(\zeta_{2}\right), \\
\int_{\mathfrak{F}_{1}} & \int_{\mathfrak{F}_{2}}\left(\mathfrak{D}_{0} f\right)\left(\zeta_{1}, \zeta_{2}\right) d E_{1}\left(\zeta_{1}\right) R_{2} d E_{2}\left(\zeta_{2}\right) \\
& =\int_{\mathfrak{F}_{1}} \int_{\mathfrak{F}_{2}} \zeta_{2}\left(\mathfrak{D}_{0} f\right)\left(\zeta_{1}, \zeta_{2}\right) d E_{1}\left(\zeta_{1}\right) R d E_{2}\left(\zeta_{2}\right) .
\end{aligned}
$$

Поскольку $\left(\zeta_{1}-\zeta_{2}\right)\left(\mathfrak{D}_{0} f\right)\left(\zeta_{1}, \zeta_{2}\right)=f\left(\zeta_{1}\right)-f\left(\zeta_{2}\right), \zeta_{1} \in \mathfrak{F}_{1}, \zeta_{2} \in \mathfrak{F}_{2}$, получаем

$$
\begin{aligned}
\int_{\mathfrak{F}_{1}} & \int_{\mathfrak{F}_{2}}\left(\mathfrak{D}_{0} f\right)\left(\zeta_{1}, \zeta_{2}\right) d E_{1}\left(\zeta_{1}\right)\left(N_{1} R-R N_{2}\right) d E_{2}\left(\zeta_{2}\right) \\
& =\int_{\mathfrak{F}_{1}} \int_{\mathfrak{F}_{2}} f\left(\zeta_{1}\right) d E_{1}\left(\zeta_{1}\right) R d E_{2}\left(\zeta_{2}\right)-\int_{\mathfrak{F}_{1}} \int_{\mathfrak{F}_{2}} f\left(\zeta_{2}\right) d E_{1}\left(\zeta_{1}\right) R d E_{2}\left(\zeta_{2}\right) .
\end{aligned}
$$

Также из определения двойных операторных интегралов легко видеть, что

$$
\begin{aligned}
& \int_{\mathfrak{F}_{1}} \int_{\mathfrak{F}_{2}} f\left(\zeta_{1}\right) d E_{1}\left(\zeta_{1}\right) R d E_{2}\left(\zeta_{2}\right)=\left(\int_{\mathfrak{F}_{1}} f\left(\zeta_{1}\right) d E_{1}\left(\zeta_{1}\right)\right) R=f\left(N_{1}\right) R, \\
& \int_{\mathfrak{F}_{1}} \int_{\mathfrak{F}_{2}} f\left(\zeta_{2}\right) d E_{1}\left(\zeta_{1}\right) R d E_{2}\left(\zeta_{2}\right)=R \int_{\mathfrak{F}_{1}} f\left(\zeta_{1}\right) d E_{1}\left(\zeta_{1}\right)=R f\left(N_{2}\right),
\end{aligned}
$$

откуда вытекает равенство (3.5.2).

Пусть теперь $N_{1}$ и $N_{2}$ - не обязательно ограниченные нормальные операторы. Из доказанной части теоремы 3.5.2 и из теоремы 3.2.1 вытекает коммутаторно липшицева оценка, и, стало быть, оператор $f\left(N_{1}\right) R-R f\left(N_{2}\right)$ ограничен.

Положим

$$
P_{k} \stackrel{\text { def }}{=} E_{1}(\{\zeta \in \mathbb{C}:|\zeta| \leqslant k\}), \quad Q_{k} \stackrel{\text { def }}{=} E_{2}(\{\zeta \in \mathbb{C}:|\zeta| \leqslant k\}), \quad k>0 .
$$


Тогда $N_{1, k} \stackrel{\text { def }}{=} P_{k} N_{1}$ и $N_{2, k} \stackrel{\text { def }}{=} Q_{k} N_{2}$ - ограниченные нормальные операторы. Пусть $E_{j, k}$ - спектральная мера оператора $N_{j, k}, j=1,2$. Имеем

$$
\begin{aligned}
P_{k} & \left(\int_{\mathfrak{F}_{1}} \int_{\mathfrak{F}_{2}}\left(\mathfrak{D}_{0} f\right)\left(\zeta_{1}, \zeta_{2}\right) d E_{1}\left(\zeta_{1}\right)\left(N_{1} R-R N_{2}\right) d E_{2}\left(\zeta_{2}\right)\right) Q_{k} \\
& =P_{k}\left(\int_{\mathfrak{F}_{1}} \int_{\mathfrak{F}_{2}}\left(\mathfrak{D}_{0} f\right)\left(\zeta_{1}, \zeta_{2}\right) d E_{1, k}\left(\zeta_{1}\right)\left(P_{k} f\left(N_{1}\right) R-R f\left(N_{2}\right) Q_{k}\right) d E_{2, k}\left(\zeta_{2}\right)\right) Q_{k} .
\end{aligned}
$$

Применяя (3.5.2) к ограниченным нормальным операторам $N_{1, k}$ и $N_{2, k}$, получаем

$$
\begin{aligned}
P_{k}\left(f\left(N_{1, k}\right) R-R f\left(N_{2, k}\right)\right) Q_{k} \\
\quad=P_{k}\left(\int_{\mathfrak{F}_{1}} \int_{\mathfrak{F}_{2}}\left(\mathfrak{D}_{0} f\right)\left(\zeta_{1}, \zeta_{2}\right) d E_{1, k}\left(\zeta_{1}\right)\left(P_{k} N_{1} R-R N_{2} Q_{k}\right) d E_{2, k}\left(\zeta_{2}\right)\right) Q_{k} .
\end{aligned}
$$

Поскольку $P_{k}\left(f\left(N_{1, k}\right) R-R f\left(N_{2, k}\right)\right) Q_{k}=P_{k}\left(f\left(N_{1}\right) R-R f\left(N_{2}\right)\right) Q_{k}$, имеем

$$
\begin{aligned}
& P_{k}\left(f\left(N_{1}\right) R-R f\left(N_{2}\right)\right) Q_{k} \\
& \quad=P_{k}\left(\int_{\mathfrak{F}_{1}} \int_{\mathfrak{F}_{2}}\left(\mathfrak{D}_{0} f\right)\left(\zeta_{1}, \zeta_{2}\right) d E_{1}\left(\zeta_{1}\right)\left(N_{1} R-R N_{2}\right) d E_{2}\left(\zeta_{2}\right)\right) Q_{k} .
\end{aligned}
$$

Остаётся перейти к пределу в сильной операторной топологии. Теорема доказана.

Нетрудно убедиться в том, что во всех формулах настоящего раздела функцию $\mathfrak{D}_{0} f\left(\zeta_{1}, \zeta_{2}\right)$ под знаком двойного операторного интеграла можно заменить любой ограниченной измеримой функцией $F\left(\zeta_{1}, \zeta_{2}\right)$, совпадающей с $\mathfrak{D}_{0} f\left(\zeta_{1}, \zeta_{2}\right)$ при всех $\zeta_{1}$ и $\zeta_{2}$ таких, что $\zeta_{1} \neq \zeta_{2}$.

В частности, в случае, когда $\mathfrak{F}_{1}=\mathfrak{F}_{2}$ и множество $\mathfrak{F}_{1}$ совершенно, можно, ввиду теоремы 3.3.6, в формуле (3.5.2) заменить $\mathfrak{D}_{0} f$ разделённой разностью $\mathfrak{D} f$.

ТеОРема 3.5.4. Пусть $\mathfrak{F}$ - замкнутое совершенное подмножество плоскости $\mathbb{C}$, a $f \in \mathrm{CL}(\mathfrak{F})$. Тогда если $N_{1}$ и $N_{2}$ - нормальные операторы такие, что $\sigma\left(N_{j}\right) \subset \mathfrak{F}, j=1,2$, a $R$ - ограниченныц линейный оператор, то справедлива формула

$$
f\left(N_{1}\right) R-R f\left(N_{2}\right)=\int_{\mathfrak{F}} \int_{\mathfrak{F}}(\mathfrak{D} f)\left(\zeta_{1}, \zeta_{2}\right) d E_{1}\left(\zeta_{1}\right)\left(N_{1} R-R N_{2}\right) d E_{2}\left(\zeta_{2}\right),
$$

где $E_{j}$ - спектральная мера оператора $N_{j}$.

Перейдём теперь к осмыслению результатов раздела 3.4 в свете двойных операторных интегралов. Имеет место следующее утверждение.

Теорема 3.5.5. Пусть $f$ - функиия, непрерьвная на замкнутом подмножестве $\mathfrak{F}$ комплексной плоскости $\mathbb{C}$ такая, что существуют мультипликаторы Шура $\Phi_{1}, \Phi_{2} \in \mathfrak{M}(\mathfrak{F} \times \mathfrak{F})$ такие, что

$$
f\left(\zeta_{1}\right)-f\left(\zeta_{2}\right)=\left(\zeta_{1}-\zeta_{2}\right) \Phi_{1}\left(\zeta_{1}, \zeta_{2}\right)+\left(\bar{\zeta}_{1}-\bar{\zeta}_{2}\right) \Phi_{2}\left(\zeta_{1}, \zeta_{2}\right), \quad \zeta_{1}, \zeta_{2} \in \mathfrak{F}
$$


Пусть $N_{1}$ и $N_{2}$ - нормальные операторы, спектры которых содержатся в $\mathfrak{F}$. Тогда

$$
\begin{aligned}
f\left(N_{1}\right)-f\left(N_{2}\right)= & \int_{\mathfrak{F}} \int_{\mathfrak{F}} \Phi_{1}\left(\zeta_{1}, \zeta_{2}\right) d E_{1}\left(\zeta_{1}\right)\left(N_{1}-N_{2}\right) d E_{2}\left(\zeta_{2}\right) \\
& +\int_{\mathfrak{F}} \int_{\mathfrak{F}} \Phi_{2}\left(\zeta_{1}, \zeta_{2}\right)\left(\zeta_{1}, \zeta_{2}\right) d E_{1}\left(\zeta_{1}\right)\left(N_{1}^{*}-N_{2}^{*}\right) d E_{2}\left(\zeta_{2}\right) .
\end{aligned}
$$

ЗАмечАниЕ. Из (3.5.5) легко следует, что $\left\|f\left(N_{1}\right)-f\left(N_{2}\right)\right\| \leqslant\left(\left\|\Phi_{1}\right\|_{\mathfrak{M}(\mathfrak{F} \times \mathfrak{F})}+\right.$ $\left.\left\|\Phi_{2}\right\|_{\mathfrak{M}(\mathfrak{F} \times \mathfrak{F})}\right)\left\|N_{1}-N_{2}\right\|$ и, в частности, $f$ - операторно липшицева функция.

ДоКАЗАТЕЛЬСтво тЕОРЕмы 3.5.5. Как и в доказательстве теоремы 3.5.2, предположим сначала, что операторы $N_{1}$ и $N_{2}$ ограничены. Тогда

$$
\begin{aligned}
\int_{\mathfrak{F}} & \int_{\mathfrak{F}} \Phi_{1}\left(\zeta_{1}, \zeta_{2}\right) d E_{1}\left(\zeta_{1}\right)\left(N_{1}-N_{2}\right) d E_{2}\left(\zeta_{2}\right) \\
= & \int_{\mathfrak{F}} \int_{\mathfrak{F}} \Phi_{1}\left(\zeta_{1}, \zeta_{2}\right) d E_{1}\left(\zeta_{1}\right) N_{1} d E_{2}\left(\zeta_{2}\right)-\int_{\mathfrak{F}} \int_{\mathfrak{F}} \Phi_{1}\left(\zeta_{1}, \zeta_{2}\right) d E_{1}\left(\zeta_{1}\right) N_{2} d E_{2}\left(\zeta_{2}\right) \\
& =\int_{\mathfrak{F}} \int_{\mathfrak{F}} \zeta_{1} \Phi_{1}\left(\zeta_{1}, \zeta_{2}\right) d E_{1}\left(\zeta_{1}\right) d E_{2}\left(\zeta_{2}\right)-\int_{\mathfrak{F}} \int_{\mathfrak{F}} \zeta_{2} \Phi_{1}\left(\zeta_{1}, \zeta_{2}\right) d E_{1}\left(\zeta_{1}\right) d E_{2}\left(\zeta_{2}\right) \\
& =\int_{\mathfrak{F}} \int_{\mathfrak{F}}\left(\zeta_{1}-\zeta_{2}\right) \Phi_{1}\left(\zeta_{1}, \zeta_{2}\right) d E_{1}\left(\zeta_{1}\right) d E_{2}\left(\zeta_{2}\right) .
\end{aligned}
$$

Аналогично,

$$
\begin{gathered}
\int_{\mathfrak{F}} \int_{\mathfrak{F}} \Phi_{2}\left(\zeta_{1}, \zeta_{2}\right)\left(\zeta_{1}, \zeta_{2}\right) d E_{1}\left(\zeta_{1}\right)\left(N_{1}^{*}-N_{2}^{*}\right) d E_{2}\left(\zeta_{2}\right) \\
=\int_{\mathfrak{F}} \int_{\mathfrak{F}}\left(\bar{\zeta}_{1}-\bar{\zeta}_{2}\right) \Phi_{2}\left(\zeta_{1}, \zeta_{2}\right) d E_{1}\left(\zeta_{1}\right) d E_{2}\left(\zeta_{2}\right) .
\end{gathered}
$$

Таким образом, правая часть равенства (3.5.5) равна

$$
\begin{aligned}
& \int_{\mathfrak{F}} \int_{\mathfrak{F}}\left(f\left(\zeta_{1}\right)-f\left(\zeta_{2}\right)\right) d E_{1}\left(\zeta_{1}\right) d E_{2}\left(\zeta_{2}\right) \\
& \quad=\int_{\mathfrak{F}} f\left(\zeta_{1}\right) d E_{1}\left(\zeta_{1}\right)-\int_{\mathfrak{F}} f\left(\zeta_{2}\right) d E_{2}\left(\zeta_{2}\right)=f\left(N_{1}\right)-f\left(N_{2}\right) .
\end{aligned}
$$

Переход от ограниченных операторов к неограниченным осуществляется так же, как в доказательстве теоремы 3.5.2. Теорема 3.5.5 доказана.

Перейдём теперь к применению двойных операторных интегралов в задачах операторной дифференцируемости.

Теорема 3.5.6. Пусть $f$-операторно липшищева функция на $\mathbb{R}$, a $A$ и $K$ самосопряжённые операторы, причём оператор $K$ ограничен. Тогда

$$
\lim _{t \rightarrow 0} \frac{1}{t}(f(A+t K)-f(A))=\int_{\mathbb{R}} \int_{\mathbb{R}}(\mathfrak{D} f)(x, y) d E_{A}(x) K d E_{A}(y),
$$

где предел берётся в сильной операторной топологии.

Нам понадобится несколько вспомогательных утверждений. Пусть $\widehat{\mathbb{R}} \stackrel{\text { def }}{=}$ $\mathbb{R} \cup\{\infty\}$ обозначает одноточечную компактификацию вещественной прямой $\mathbb{R}$. Напомним, что любая функция $f \in \mathrm{OL}(\mathbb{R})$ дифференцируема всюду на $\widehat{\mathbb{R}}$ (см. теорему 3.3 .3 ). 
Лемма 3.5.7. Пусть $f \in \mathrm{OL}(\mathbb{R})$. Тогда существуют две последовательности непрерывных на $\widehat{\mathbb{R}}$ буни,ий $\left\{\varphi_{n}\right\}_{n \geqslant 0} u\left\{\psi_{n}\right\}_{n \geqslant 0}$ maкие, что

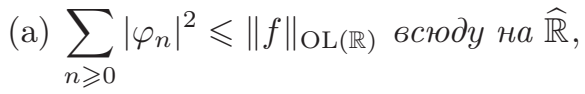
(b) $\sum_{n \geqslant 0}\left|\psi_{n}\right|^{2} \leqslant\|f\|_{\mathrm{OL}(\mathbb{R})}$ всюоу на $\widehat{\mathbb{R}}$,
(c) $(\mathfrak{D} f)(x, y)=\sum_{n \geqslant 0} \varphi_{n}(x) \psi_{n}(y)$ nри всех $x, y \in \mathbb{R}$.

ДокАЗАТЕЛьство. В силу теоремы 3.3.6 мы имеем: $\mathfrak{D} f \in \mathfrak{M}(\mathbb{R} \times \mathbb{R})$ и $\|\mathfrak{D} f\|_{\mathfrak{M}(\mathbb{R} \times \mathbb{R})}=\|f\|_{\mathrm{OL}(\mathbb{R})}$. Продолжим функцию $\mathfrak{D} f$ на множество $\widehat{\mathbb{R}} \times \widehat{\mathbb{R}}$, положив $(\mathfrak{D} f)(x, y)=f^{\prime}(\infty)=\lim _{t \rightarrow \infty} t^{-1} f(t)$ в случае, когда $|x|+|y|=\infty$. Ясно что эта продолженная на $\widehat{\mathbb{R}} \times \widehat{\mathbb{R}}$ функция $\mathfrak{D} f$ непрерывна по каждой переменной. Отсюда получаем:

$$
\begin{aligned}
\|\mathfrak{D} f\|_{\mathfrak{M}(\widehat{\mathbb{R}} \times \widehat{\mathbb{R}})} & =\sup \left\{\|\mathfrak{D} f\|_{\mathfrak{M}\left(\Lambda_{1} \times \Lambda_{2}\right)}: \Lambda_{1}, \Lambda_{2} \subset \widehat{\mathbb{R}}, \Lambda_{1} \text { и } \Lambda_{2} \text { конечны }\right\} \\
& =\sup \left\{\|\mathfrak{D} f\|_{\mathfrak{M}\left(\Lambda_{1} \times \Lambda_{2}\right)}: \Lambda_{1}, \Lambda_{2} \subset \mathbb{R}, \Lambda_{1} \text { и } \Lambda_{2} \text { конечны }\right\} \\
& =\|\mathfrak{D} f\|_{\mathfrak{M}(\mathbb{R} \times \mathbb{R})} .
\end{aligned}
$$

Остаётся применить теорему 2.2 .4 к функции $\mathfrak{D} f: \widehat{\mathbb{R}} \times \widehat{\mathbb{R}} \rightarrow \mathbb{C}$. Лемма доказана.

Лемма 3.5.8. Пусть А и $K$ - самосопряжённые операторь, причём оператор $K$ ограничен. Тогда для любой функции $f$ из $C(\widehat{\mathbb{R}})$ функиия $H, H(t)=$ $f(A+t K)$, действует непрерьвно из $\mathbb{R}$ в пространство $\mathcal{B}(\mathscr{H})$ с нормированной топологией.

Отметим, что в работе [8] получено существенно более сильное утверждение.

ДокАЗАтельство. Можно считать, что $f(\infty)=0$. Тогда мы можем построить последовательность $\left\{f_{n}\right\}_{n \geqslant 0}$ функций класса $C^{\infty}$ с компактным носителем такую, что $f_{n} \rightarrow f$ равномерно. Каждая функция $H_{n}, H_{n}(t)=f_{n}(A+t K)$, непрерывна, поскольку $f_{n} \in \mathrm{OL}(\mathbb{R})$ при $n \geqslant 0$. Остаётся заметить, что $H_{n} \rightarrow H$ равномерно.

Лемма 3.5.9. Пусть $\left\{X_{n}\right\}_{n \geqslant 0}$ - последовательность в пространстве операторов $\mathcal{B}(\mathscr{H})$, а $\left\{u_{n}\right\}_{n \geqslant 0}-$ последовательность в гильбертовом пространстве $\mathscr{H}$. Предположим, что $\sum_{n \geqslant 0} X_{n} X_{n}^{*} \leqslant a^{2} I$ u $\sum_{n \geqslant 0}\left\|u_{n}\right\|^{2} \leqslant b^{2}$ для некоторых неотрицательных чисел а и

$$
\left\|\sum_{n \geqslant 0} X_{n} u_{n}\right\| \leqslant a b
$$

ДоказАТЕЛЬСтво. Пусть $v \in \mathscr{H}$ и $\|v\|=1$. Тогда

$$
\sum_{n \geqslant 0}\left|\left(X_{n} u_{n}, v\right)\right|=\sum_{n \geqslant 0}\left|\left(u_{n}, X_{n}^{*} v\right)\right| \leqslant\left(\sum_{n \geqslant 0}\left\|u_{n}\right\|^{2}\right)^{1 / 2}\left(\sum_{n \geqslant 0}\left\|X_{n}^{*} v\right\|^{2}\right)^{1 / 2} \leqslant a b,
$$

откуда следует доказываемое утверждение. 
ДОКАЗАТЕЛЬСТВО ТЕОРЕМЫ 3.5.6. В силу формул (3.5.4) и (2.3.5) теорему 3.5.6 можно переформулировать следующим образом:

$$
\lim _{t \rightarrow 0} \sum_{n \geqslant 0} \varphi_{n}(A+t K) K \psi_{n}(A)=\sum_{n \geqslant 0} \varphi_{n}(A) K \psi_{n}(A)
$$

в сильной операторной топологии, где $\varphi_{n}$ и $\psi_{n}$ обозначают функции, существование которых утверждается в лемме 3.5.7. Другими словами, нам нужно доказать, что для любого вектора $u \in \mathscr{H}$

$$
\lim _{t \rightarrow 0} \sum_{n \geqslant 0}\left(\varphi_{n}(A+t K)-\varphi_{n}(A)\right) K \psi_{n}(A) u=\mathbf{0},
$$

где ряд суммируется в слабой топологии пространства $\mathscr{H}$, а предел берётся в пространстве $\mathscr{H}$ по норме. Будем считать, что $\|u\|=1$ и $\|f\|_{\mathrm{OL}(\mathbb{R})}=1$. Тогда $\sum_{n \geqslant 0}\left|\varphi_{n}\right|^{2} \leqslant 1$ и $\sum_{n \geqslant 0}\left|\psi_{n}\right|^{2} \leqslant 1$ всюду на $\mathbb{R}$.

Положим $u_{n} \stackrel{\text { def }}{=} K \psi_{n}(A) u$. Имеем:

$$
\sum_{n \geqslant 0}\left\|u_{n}\right\|^{2} \leqslant\|K\|^{2} \sum_{n \geqslant 0}\left\|\psi_{n}(A) u\right\|^{2}=\|K\|^{2} \sum_{n \geqslant 0}\left(\left|\psi_{n}\right|^{2}(A) u, u\right) \leqslant\|K\|^{2}<+\infty .
$$

Пусть $\varepsilon>0$. Выберем натуральное число $N$ так, чтобы $\sum_{n>N}\left\|u_{n}\right\|^{2}<\varepsilon^{2}$. Тогда из леммы 3.5.9 следует, что

$$
\left\|\sum_{n>N}\left(\varphi_{n}(A+t K)-\varphi_{n}(A)\right) u_{n}\right\| \leqslant 2 \varepsilon
$$

при всех $t \in \mathbb{R}$. Заметим, что

$$
\left\|\sum_{n=0}^{N}\left(\varphi_{n}(A+t K)-\varphi_{n}(A)\right) u_{n}\right\| \leqslant\|K\| \sum_{n=0}^{N}\left\|\varphi_{n}(A+t K)-\varphi_{n}(A)\right\|<\varepsilon
$$

при всех достаточно близких к нулю $t$ в силу леммы 3.5.8. Таким образом, $\left\|\sum_{n \geqslant 0}\left(\varphi_{n}(A+t K)-\varphi_{n}(A)\right) u_{n}\right\|<3 \varepsilon$ при всех достаточно близких к нулю $t$. Теорема доказана.

Аналогично теореме 3.5.6 можно доказать следующую теорему.

Теорема 3.5.10. Пусть А и K - ограниченные самосопряжённые операторъ. Тогда

$$
\lim _{t \rightarrow 0} \frac{1}{t}(f(A+t K)-f(A))=\int_{\mathbb{R}} \int_{\mathbb{R}}(\mathfrak{D} f)(x, y) d E_{A}(x) K d E_{A}(y)
$$

при всех $f$ из $\mathrm{OL}_{\mathrm{loc}}(\mathbb{R})$, где предел берётся в сильной операторной топологии.

Из теоремы 3.5.6 вытекает следующее утверждение. 
Теорема 3.5.11. Пусть $f$ - операторно дифферениируемая функиия на $\mathbb{R}$, а $A$ и $K$ - самосопряжённые операторы, причём оператор $K$ ограничен. Тогда для производной функиии $t \mapsto f(A+t K)-f(A)$ по операторной норме справедлива формула

$$
\left.\frac{d}{d t}(f(A+t K)-f(A))\right|_{t=0}=\int_{\mathbb{R}} \int_{\mathbb{R}} \frac{f(x)-f(y)}{x-y} d E_{A}(x) K d E_{A}(y) .
$$

В частности, формула (3.5.6) справедлива для любой функции $f$ класса Бесова $B_{\infty, 1}^{1}(\mathbb{R})$ (см. теорему 1.6.4).

Аналогичные утверждения справедливы и для функций на окружности.

\section{6. Ядерная липшицевость и ядерно-коммутаторная липшицевость}

Цель этого раздела состоит в том, чтобы доказать, что для произвольного замкнутого множества $\mathfrak{F}$ на плоскости классы $\mathrm{CL}(\mathfrak{F})$ и $\mathrm{CL}_{\boldsymbol{S}_{1}}(\mathfrak{F})$ совпадают. В частности, если $\mathfrak{F} \subset \mathbb{R}$, то $\mathrm{OL}(\mathfrak{F})=\mathrm{OL}_{\boldsymbol{S}_{1}}(\mathfrak{F})$ (см. раздел 3.1 , где определяются классы $\mathrm{CL}_{\boldsymbol{S}_{1}}(\mathfrak{F})$ и $\left.\mathrm{OL}_{\boldsymbol{S}_{1}}(\mathfrak{F})\right)$.

Заметим, что определение класса $\mathrm{CL}_{\boldsymbol{S}_{1}}(\mathfrak{F})$ естественным образом обобщается до определения класса $\mathrm{CL}_{\boldsymbol{S}_{1}}\left(\mathfrak{F}_{1}, \mathfrak{F}_{2}\right)$, где $\mathfrak{F}_{1}$ и $\mathfrak{F}_{2}-$ непустые замкнутые подмножества комплексной плоскости $\mathbb{C}$.

Лемма 3.6.1. Пусть $f-$ непрерьвная функиия на обгединении $\mathfrak{F}_{1} \cup \mathfrak{F}_{2}$ замкнутых подмножеств $\mathfrak{F}_{1}$ u $\mathfrak{F}_{2}$ комплексной плоскости $\mathbb{C}$. Тогда

$$
\|f\|_{\mathrm{CL}_{S_{1}}\left(\mathfrak{F}_{1}, \mathfrak{F}_{2}\right)} \geqslant\left\|\mathfrak{D}_{0} f\right\|_{\mathfrak{M}_{0, S_{1}}\left(\mathfrak{F}_{1} \times \mathfrak{F}_{2}\right)} \geqslant \frac{1}{2}\left\|\mathfrak{D}_{0} f\right\|_{\mathfrak{M}_{\left(\mathfrak{F}_{1} \times \mathfrak{F}_{2}\right)}}
$$

ДокАзАтЕльство. Второе неравенство вытекает из следствия 2.1.5. Докажем первое. Достаточно рассмотреть случай конечных множеств $\mathfrak{F}_{1}$ и $\mathfrak{F}_{2}$. Пусть $N_{1}$ и $N_{2}$ - нормальные операторы с простыми спектрами такими, что $\sigma\left(N_{1}\right)=\mathfrak{F}_{1}$ и $\sigma\left(N_{2}\right)=\mathfrak{F}_{2}$. Тогда в $\mathscr{H}_{1}$ и $\mathscr{H}_{2}$ существуют ортонормированные базисы $\left\{u_{\lambda}\right\}_{\lambda \in \sigma\left(N_{1}\right)}$ и $\left\{v_{\mu}\right\}_{\mu \in \sigma\left(N_{2}\right)}$ такие, что $N_{1} u_{\lambda}=\lambda u_{\lambda}$ при $\lambda \in \sigma\left(N_{1}\right)$ и $N_{2} v_{\mu}=\mu v_{\mu}$ при $\mu \in \sigma\left(N_{2}\right)$. С каждым оператором $X: \mathscr{H}_{2} \rightarrow \mathscr{H}_{1}$ мы связываем матрицу $\left\{\left(X v_{\mu}, u_{\lambda}\right)\right\}_{(\lambda, \mu) \in \sigma\left(N_{1}\right) \times \sigma\left(N_{2}\right)}$. Имеем

$$
\left(\left(N_{1} R-R N_{2}\right) v_{\mu}, u_{\lambda}\right)=\left(R v_{\mu}, N_{1}^{*} u_{\lambda}\right)-\left(R N_{2} v_{\mu}, u_{\lambda}\right)=(\lambda-\mu)\left(R v_{\mu}, u_{\lambda}\right) .
$$

Аналогично, $\left(\left(f\left(N_{1}\right) R-R f\left(N_{2}\right)\right) v_{\mu}, u_{\lambda}\right)=(f(\lambda)-f(\mu))\left(R v_{\mu}, u_{\lambda}\right)$. Ясно, что $\left\{(f(\lambda)-f(\mu))\left(R v_{\mu}, u_{\lambda}\right)\right\}=\left\{\left(\mathfrak{D}_{0} f\right)(\lambda, \mu)\right\} \star\left\{(\lambda-\mu)\left(R v_{\mu}, u_{\lambda}\right)\right\}$. Заметим, что матрица $\left\{a_{\lambda \mu}\right\}_{(\lambda, \mu) \in \sigma\left(N_{1}\right) \times \sigma\left(N_{2}\right)}$ представима в виде

$$
\left\{a_{\lambda \mu}\right\}_{(\lambda, \mu) \in \sigma\left(N_{1}\right) \times \sigma\left(N_{2}\right)}=\left\{(\lambda-\mu)\left(R v_{\mu}, u_{\lambda}\right)\right\}_{(\lambda, \mu) \in \sigma\left(N_{1}\right) \times \sigma\left(N_{2}\right)},
$$

где $R$ - оператор, действующий из $\mathscr{H}_{2}$ в $\mathscr{H}_{1}$, в том и только том случае, когда $a_{\lambda \mu}=0$ при $\lambda=\mu$. Теперь неравенство $\|f\|_{\mathrm{CL}\left(\mathfrak{F}_{1}, \mathfrak{F}_{2}\right)} \geqslant\left\|\mathfrak{D}_{0} f\right\|_{\mathfrak{M}_{0, \mathfrak{S}_{1}}\left(\mathfrak{F}_{1} \times \mathfrak{F}_{2}\right)}$ очевидно. Лемма доказана.

СлЕДСтвиЕ 3.6.2. Пусть $f$ - вещественная непрерьвная функиия на замкнутом подмножестве $\mathfrak{F}$ вещественной прямой $\mathbb{R}$. Тогда

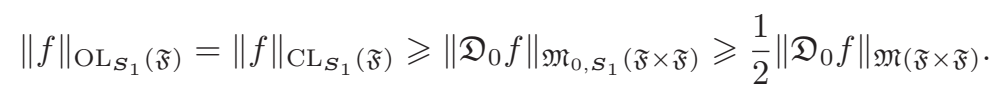


Если отказаться от вещественности функции $f$, то

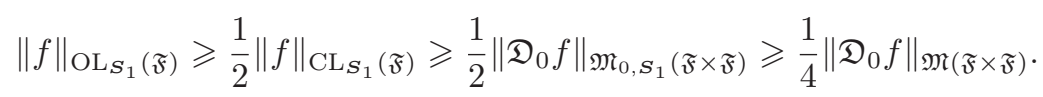

ДоказАтельство. Равенство в (3.6.1) вытекает из следствия 3.1.7. Все неравенства в (3.6.1) уже доказаны выше. Очевидно, что (3.6.2) следует из (3.6.1).

Перейдём к основным результатам этого раздела.

ТЕОРема 3.6.3. Пусть $\mathfrak{F}$ - замкнутое множество в $\mathbb{C}$. Тогда $\mathrm{CL}(\mathfrak{F})=$ $\mathrm{CL}_{\boldsymbol{S}_{1}}(\mathfrak{F}) u$

$$
\frac{1}{2}\|f\|_{\mathrm{CL}_{S_{1}}(\mathfrak{F})} \leqslant\|f\|_{\mathrm{CL}(\mathfrak{F})} \leqslant 2\|f\|_{\mathrm{CL}_{S_{1}}(\mathfrak{F})}, \quad f \in \mathrm{CL}_{(\mathfrak{F}) .}
$$

ДоказАтельство. Пусть $f \in \mathrm{CL}(\mathfrak{F})$, и пусть $N_{1}$ и $N_{2}$ - нормальные операторы со спектрами в $\mathfrak{F}$ такие, что $N_{1} R-R N_{2} \in \boldsymbol{S}_{1}$, а $R$ - ограниченный оператор. Тогда, ввиду замечания к теореме 3.5.2,

$$
\begin{aligned}
\left\|f\left(N_{1}\right) R-R f\left(N_{2}\right)\right\|_{S_{1}} & \leqslant\left\|\mathfrak{D}_{0} f\right\|_{\mathfrak{M}\left(E_{1}, E_{2}\right)}\left\|N_{1} R-R N_{2}\right\|_{S_{1}} \\
& \leqslant\left\|\mathfrak{D}_{0} f\right\|_{\mathfrak{M}(\mathfrak{F} \times \mathfrak{F})}\left\|N_{1} R-R N_{2}\right\|_{S_{1}} \\
& \leqslant 2\|f\|_{\mathrm{CL}(\mathfrak{F})}\left\|N_{1} R-R N_{2}\right\|_{S_{1}}
\end{aligned}
$$

в силу теоремы 3.3.2. Отсюда получаем неравенство $\|f\|_{\mathrm{CL}_{S_{1}}(\mathfrak{F})} \leqslant 2\|f\|_{\mathrm{CL}(\mathfrak{F})}$. С другой стороны, из леммы 3.6.1 и из теоремы 3.3.2 получаем

$$
\|f\|_{\mathrm{CL}(\mathfrak{F})} \leqslant\left\|\mathfrak{D}_{0} f\right\|_{\mathfrak{M}(\mathfrak{F} \times \mathfrak{F})} \leqslant 2\|f\|_{\mathrm{CL}_{S_{1}(\mathfrak{F})}} .
$$

Теорема 3.6.3 доказана.

Если $\mathfrak{F}$ - совершенное множество, можно получить более точный результат.

Теорема 3.6.4. Пусть $\mathfrak{F}$ - замкнутое совершенное множество в $\mathbb{C}$. Тогда $\|f\|_{\mathrm{CL}_{S_{1}}(\mathfrak{F})}=\|f\|_{\mathrm{CL}(\mathfrak{F})}$ для всех $f$ из $\mathrm{CL}(\mathfrak{F})=\mathrm{CL}_{\boldsymbol{S}_{1}}(\mathfrak{F})$.

ДокАзАтельСтво. В силу формулы (3.5.4) при $f \in \mathrm{CL}(\mathfrak{F})$ имеем

$$
\begin{aligned}
\left\|f\left(N_{1}\right) R-R f\left(N_{2}\right)\right\|_{S_{1}} & \leqslant\|\mathfrak{D} f\|_{\mathfrak{M}\left(E_{1}, E_{2}\right)}\left\|N_{1} R-R N_{2}\right\|_{S_{1}} \\
& \leqslant\|\mathfrak{D} f\|_{\mathfrak{M}(\mathfrak{F} \times \mathfrak{F})}\left\|N_{1} R-R N_{2}\right\|_{S_{1}} \\
& =\|f\|_{\mathrm{CL}(\mathfrak{F})}\left\|N_{1} R-R N_{2}\right\|_{S_{1}} .
\end{aligned}
$$

Последнее равенство гарантируется теоремой 3.3.6. Тем самым мы доказали, что $\|f\|_{\mathrm{CL}_{S_{1}}(\mathfrak{F})} \leqslant\|f\|_{\mathrm{CL}(\mathfrak{F})}$. Применяя теперь леммы 3.6.1 и 2.1.6, а также теорему 3.3.6, получаем:

$$
\|f\|_{\mathrm{CL}_{S_{1}}(\mathfrak{F})} \geqslant\left\|\mathfrak{D}_{0} f\right\|_{\mathfrak{M}_{0, S_{1}}(\mathfrak{F} \times \mathfrak{F})}=\|\mathfrak{D} f\|_{\mathfrak{M}(\mathfrak{F} \times \mathfrak{F})}=\|f\|_{\mathrm{CL}(\mathfrak{F})} .
$$

Теорема доказана.

Настало время перейти к центральному результату этого раздела.

Теорема 3.6.5. Пусть $f$-непрерывная функция на $\mathbb{R}$. Следующие условия эквивалентны:

(a) функция $f$ операторно липшищева;

(b) функиия $f$ ядерно липшицева;

(c) $f(A)-f(B) \in \boldsymbol{S}_{1}$, как толъко $A$ и $B$ - самосопряжённые операторы с разностъю $A-B$ из $\boldsymbol{S}_{1}$. 
Отметим, что в утверждении (с) необходимо рассматривать не только ограниченные операторы $A$ и $B$.

ДокАЗАтЕльство. Эквивалентность условий (a) и (b) установлена в следствии 3.6.2. Импликация (b) $\Rightarrow$ (c) тривиальна. Покажем, что (c) $\Rightarrow$ (b). Предположим, что $f \notin \mathrm{CL}_{S_{1}}(\mathbb{R})$. Тогда можно найти последовательности самосопряжённых операторов $A_{n}$ и $B_{n}$ такие, что $A_{n}-B_{n} \in \boldsymbol{S}_{1}$ и $\left\|A_{n}-B_{n}\right\|_{\boldsymbol{S}_{1}}^{-1} \| f\left(A_{n}\right)-$ $f\left(B_{n}\right) \|_{\boldsymbol{S}_{1}} \rightarrow \infty$, когда $n \rightarrow \infty$. Не умаляя общности, можно считать, что $\lim _{n \rightarrow \infty}\left\|A_{n}-B_{n}\right\|_{S_{1}}=0$. Действительно, рассмотрим приращение $A_{n} \mapsto$ $A_{n}+K_{n}$, где $K_{n} \stackrel{\text { def }}{=} B_{n}-A_{n}$. Рассмотрим теперь следующие приращения: $A_{n}+\left(j / M_{n}\right) K_{n} \mapsto A_{n}+\left((j+1) / M_{n}\right) K_{n}, 0 \leqslant j \leqslant M_{n}-1$, где $\left\{M_{n}\right\}$ - последовательность натуральных чисел такая, что $\lim _{n \rightarrow \infty}\left\|A_{n}-B_{n}\right\|_{S_{1}} / M_{n}=0$. Выберем теперь такое число $j$, которое максимизирует число

$$
\left\|f\left(A_{n}+\frac{j+1}{M_{n}} K_{n}\right)-f\left(A_{n}+\frac{j}{M_{n}} K_{n}\right)\right\|_{S_{1}},
$$

и заменим пару $\left(A_{n}, B_{n}\right)$ парой $A_{n}+\left(j / M_{n}\right) K_{n}, A_{n}+\left((j+1) / M_{n}\right) K_{n}$. Тогда

$$
\lim _{n \rightarrow \infty}\left\|A_{n}-B_{n}\right\|_{\boldsymbol{S}_{1}}^{-1}\left\|f\left(A_{n}\right)-f\left(B_{n}\right)\right\|_{\boldsymbol{S}_{1}}=\infty \quad \text { и } \quad \lim _{n \rightarrow \infty}\left\|A_{n}-B_{n}\right\|_{\boldsymbol{S}_{1}}=0 .
$$

Теперь достаточно, если потребуется, выбрать подпоследовательность последовательности $\left(A_{n}, B_{n}\right)$ или повторить некоторые члены этой последовательности, чтобы добиться выполнения условия

$$
\sum_{n}\left\|B_{n}-A_{n}\right\|_{S_{1}}<\infty, \quad \text { но } \quad \sum_{n}\left\|f\left(B_{n}\right)-f\left(A_{n}\right)\right\|_{S_{1}}=\infty .
$$

Пусть $A$ - ортогональная сумма операторов $A_{n}$, а $B$ - ортогональная сумма операторов $B_{n}$. Тогда $B-A \in \boldsymbol{S}_{1}$, а $f(B)-f(A) \notin \boldsymbol{S}_{1}$. Теорема доказана.

ЗАмечАниЕ. Аналогичное утверждение справедливо для функций на единичной окружности и унитарных операторов.

\section{7. Операторно липшицевы функции на плоскости. Достаточное условие}

В этом разделе мы получим достаточное условие для операторной липшицевости в терминах класса Бесова $B_{\infty, 1}^{1}\left(\mathbb{R}^{2}\right)$. Оно аналогично теореме 1.6.1 для функций на вещественной прямой. Результаты этого раздела получены в работе [14].

Напомним (см. формулу (3.5.3)), что в случае функций на прямой операторная липшицевость функции $f$ может быть получена из формулы

$$
f(A)-f(B)=\int_{\mathbb{R}} \int_{\mathbb{R}} \frac{f(s)-f(t)}{s-t} d E_{A}(s)(A-B) d E_{B}(t) .
$$

Здесь $A$ и $B$ - самосопряжённые операторы. Именно так была первоначально установлена операторная липшицевость функций класса $B_{\infty, 1}^{1}(\mathbb{R})$ в [56] и [58].

Естественно было бы попробовать поступить так же и с функциями на плоскости. Однако (см. следствие 3.3.5), если разделённая разность является мультипликатором Шура для любых спектральных мер на $\mathbb{C}$, то функция должна быть линейной. 
В работе [14] был применён другой метод: для нормальных операторов $N_{1}$ и $N_{2}$ разность $f\left(N_{1}\right)-f\left(N_{2}\right)$ представляется в виде суммы двойных операторных интегралов. При этом интегрируются разделённые разности по каждой переменной.

Введём следующие обозначения. Пусть $N_{1}$ и $N_{2}$ - нормальные операторы в гильбертовом пространстве. Положим $A_{j} \stackrel{\text { def }}{=} \operatorname{Re} N_{j}, B_{j} \stackrel{\text { def }}{=} \operatorname{Im} N_{j}, j=1,2$, и пусть $E_{j}-$ спектральная мера оператора $N_{j}$. Другими словами, $N_{j}=A_{j}+\mathrm{i} B_{j}$, $j=1,2$, где $A_{j}$ и $B_{j}$ - коммутирующие самосопряжённые операторы.

Если $f$ - функция на $\mathbb{R}^{2}$, имеющая всюду частные производные по обеим переменным, рассмотрим разделённые разности по каждой переменной:

$$
\begin{aligned}
& \left(\mathfrak{D}_{x} f\right)\left(z_{1}, z_{2}\right) \stackrel{\text { def }}{=} \frac{f\left(x_{1}, y_{2}\right)-f\left(x_{2}, y_{2}\right)}{x_{1}-x_{2}}, \quad z_{1}, z_{2} \in \mathbb{C}, \\
& \left(\mathfrak{D}_{y} f\right)\left(z_{1}, z_{2}\right) \stackrel{\text { def }}{=} \frac{f\left(x_{1}, y_{1}\right)-f\left(x_{1}, y_{2}\right)}{y_{1}-y_{2}}, \quad z_{1}, z_{2} \in \mathbb{C},
\end{aligned}
$$

где $x_{j} \stackrel{\text { def }}{=} \operatorname{Re} z_{j}, y_{j} \stackrel{\text { def }}{=} \operatorname{Im} z_{j}, j=1,2$. На множествах $\left\{\left(z_{1}, z_{2}\right): x_{1}=x_{2}\right\}$ и $\left\{\left(z_{1}, z_{2}\right): y_{1}=y_{2}\right\}$ разделённые разности будут пониматься как соответствующие частные производные функции $f$.

Следующий результат даёт ключевую оценку.

Теорема 3.7.1. Пусть $f$-непрерывная ограниченная функиия на $\mathbb{R}^{2}$, преобразование Фуръе $\mathscr{F} f$ которой имеет компактный носитель. Тогда $\mathfrak{D}_{x} f$ u $\mathfrak{D}_{y} f$ являются мультипликаторами Шура класса $\mathfrak{M}(\mathbb{C} \times \mathbb{C})$. Более того, если $\operatorname{supp} \mathscr{F} f \subset\{\zeta \in \mathbb{C}:|\zeta| \leqslant \sigma\}, \sigma>0$, mo

$$
\left\|\mathfrak{D}_{x} f\right\|_{\mathfrak{M}(\mathbb{C} \times \mathbb{C})} \leqslant \text { const } \sigma\|f\|_{L^{\infty}} \quad u \quad\left\|\mathfrak{D}_{y} f\right\|_{\mathfrak{M}(\mathbb{C} \times \mathbb{C})} \leqslant \text { const } \sigma\|f\|_{L^{\infty}} .
$$

Из определения класса Бесова $B_{\infty, 1}^{1}\left(\mathbb{R}^{2}\right)$ и из теоремы 3.7.1 следует, что для любой функции $f$ класса $B_{\infty, 1}^{1}\left(\mathbb{R}^{2}\right)$ разделённые разности $\mathfrak{D}_{x} f$ и $\mathfrak{D}_{y} f$ являются мультипликаторами Шура и

$$
\left\|\mathfrak{D}_{x} f\right\|_{\mathfrak{M}(\mathbb{C} \times \mathbb{C})} \leqslant \text { const }\|f\|_{B_{\infty, 1}^{1}} \quad \text { и } \quad\left\|\mathfrak{D}_{y} f\right\|_{\mathfrak{M}(\mathbb{C} \times \mathbb{C})} \leqslant \text { const }\|f\|_{B_{\infty, 1}^{1}} .
$$

Неравенства (3.7.2) вместе с теоремой 3.5.5 влекут следующий результат, полученный в работе [14] и являющийся центральным в этом разделе.

Теорема 3.7.2. Пусть $f$ - функиия класса Бесова $B_{\infty, 1}^{1}\left(\mathbb{R}^{2}\right)$. Предположим, что $N_{1}$ и $N_{2}$ - нормальные операторы такие, что оператор $N_{1}-N_{2}$ ограничен. Тогда

$$
\begin{aligned}
f\left(N_{1}\right)-f\left(N_{2}\right)=\iint_{\mathbb{C}^{2}}\left(\mathfrak{D}_{y} f\right)\left(z_{1}, z_{2}\right) d E_{1}\left(z_{1}\right)\left(B_{1}-B_{2}\right) d E_{2}\left(z_{2}\right) \\
\quad+\iint_{\mathbb{C}^{2}}\left(\mathfrak{D}_{x} f\right)\left(z_{1}, z_{2}\right) d E_{1}\left(z_{1}\right)\left(A_{1}-A_{2}\right) d E_{2}\left(z_{2}\right)
\end{aligned}
$$

и имеет место неравенство $\left\|f\left(N_{1}\right)-f\left(N_{2}\right)\right\| \leqslant$ const $\|f\|_{B_{\infty, 1}^{1}}\left\|N_{1}-N_{2}\right\|$, m.е. $f$ - операторно липшищева функиия на $\mathbb{C}$. 
Чтобы доказать теорему 3.7.1, мы воспользуемся формулой для представления разделённой разности в виде элемента тензорного произведения Хогерупа. Напомним, что символом $\mathscr{E}_{\sigma}$ обозначается множество целых функций (одной комплексной переменной) экспоненциального типа не выше $\sigma$.

Лемма 3.7.3. Пусть $\varphi \in \mathscr{E}_{\sigma} \cap L^{\infty}(\mathbb{R})$. Тогда

$$
\frac{\varphi(x)-\varphi(y)}{x-y}=\sum_{n \in \mathbb{Z}} \sigma \frac{\varphi(x)-\varphi\left(\pi n \sigma^{-1}\right)}{\sigma x-\pi n} \frac{\sin (\sigma y-\pi n)}{\sigma y-\pi n} .
$$

Кроме того,

$$
\begin{gathered}
\sum_{n \in \mathbb{Z}} \frac{\left|\varphi(x)-\varphi\left(\pi n \sigma^{-1}\right)\right|^{2}}{(\sigma x-\pi n)^{2}} \leqslant 3\|\varphi\|_{L^{\infty}(\mathbb{R})}^{2}, \quad x \in \mathbb{R} \\
\sum_{n \in \mathbb{Z}} \frac{\sin ^{2}(\sigma y-\pi n)}{(\sigma y-\pi n)^{2}}=1, \quad y \in \mathbb{R} .
\end{gathered}
$$

Мы отсылаем читателя к работе [14], в пятом параграфе которой даётся доказательство леммы 3.7.3, основанное на формуле Котельникова-Шеннона, которая, в свою очередь, основана на том, что семейство функций

$$
\left\{(z-\pi n)^{-1} \sin (z-\pi n)\right\}_{n \in \mathbb{Z}}
$$

образует ортонормированный базис в $\mathscr{E} 1 \cap L^{2}(\mathbb{R})$ (см. [42; лекция 20, п. 3]).

ДокАЗАТЕЛЬСтво тЕОРЕмЫ 3.7.1. Ясно, что $f$ - сужение на $\mathbb{R}^{2}$ целой функции двух комплексных переменных. Более того, $f(\cdot, a), f(a, \cdot)$ принадлежит $\mathscr{E}_{\sigma} \cap L^{\infty}(\mathbb{R})$ при всех $a \in \mathbb{R}$. Не умаляя общности, мы можем предположить, что $\sigma=1$. По лемме 3.7 .3

$$
\begin{aligned}
\left(\mathfrak{D}_{x} f\right)\left(z_{1}, z_{2}\right) & =\frac{f\left(x_{1}, y_{2}\right)-f\left(x_{2}, y_{2}\right)}{x_{1}-x_{2}} \\
& =\sum_{n \in \mathbb{Z}}(-1)^{n} \frac{f\left(\pi n, y_{2}\right)-f\left(x_{2}, y_{2}\right)}{\pi n-x_{2}} \frac{\sin \left(x_{1}-\pi n\right)}{x_{1}-\pi n}, \\
\left(\mathfrak{D}_{y} f\right)\left(z_{1}, z_{2}\right) & =\frac{f\left(x_{1}, y_{1}\right)-f\left(x_{1}, y_{2}\right)}{y_{1}-y_{2}} \\
& =\sum_{n \in \mathbb{Z}}(-1)^{n} \frac{f\left(x_{1}, y_{1}\right)-f\left(x_{1}, \pi n\right)}{y_{1}-\pi n} \frac{\sin \left(y_{2}-\pi n\right)}{y_{2}-\pi n} .
\end{aligned}
$$

Заметим, что выражения $\frac{\sin \left(x_{1}-\pi n\right)}{x_{1}-\pi n}, \frac{f\left(x_{1}, y_{1}\right)-f\left(x_{1}, \pi n\right)}{y_{1}-\pi n}$ зависят от $z_{1}=$ $\left(x_{1}, y_{1}\right)$ и не зависят от $z_{2}=\left(x_{2}, y_{2}\right)$, тогда как выражения $\frac{f\left(\pi n, y_{2}\right)-f\left(x_{2}, y_{2}\right)}{\pi n-x_{2}}$, $\frac{\sin \left(y_{2}-\pi n\right)}{y_{2}-\pi n}$ зависят от $z_{2}=\left(x_{2}, y_{2}\right)$ и не зависят от $z_{1}=\left(x_{1}, y_{1}\right)$. Кроме того, 
по лемме 3.7 .3

$$
\begin{aligned}
& \sum_{n \in \mathbb{Z}} \frac{\left|f\left(x_{1}, y_{1}\right)-f\left(x_{1}, \pi n\right)\right|^{2}}{\left(y_{1}-\pi n\right)^{2}} \leqslant 3\left\|f\left(x_{1}, \cdot\right)\right\|_{L^{\infty}(\mathbb{R})}^{2} \leqslant 3\|f\|_{L^{\infty}(\mathbb{C})}^{2}, \\
& \sum_{n \in \mathbb{Z}} \frac{\left|f\left(\pi n, y_{2}\right)-f\left(x_{2}, y_{2}\right)\right|^{2}}{\left(\pi n-x_{2}\right)^{2}} \leqslant 3\left\|f\left(\cdot, y_{2}\right)\right\|_{L^{\infty}(\mathbb{R})}^{2} \leqslant 3\|f\|_{L^{\infty}(\mathbb{C})}^{2}
\end{aligned}
$$

И

$$
\sum_{n \in \mathbb{Z}} \frac{\sin ^{2}\left(x_{1}-\pi n\right)}{\left(x_{1}-\pi n\right)^{2}}=\sum_{n \in \mathbb{Z}} \frac{\sin ^{2}\left(y_{2}-\pi n\right)}{\left(y_{2}-\pi n\right)^{2}}=1,
$$

что доказывает (3.7.1). Теорема доказана.

Отметим, что неравенства (3.7.1) играют роль операторных неравенств Бернштейна (см. раздел 1.4). Так же, как в случае функций от самосопряжённых операторов, можно доказать следующие утверждения.

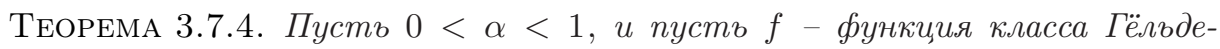
ра $\Lambda_{\alpha}\left(\mathbb{R}^{2}\right)$. Тогда для некоторой константы $с>0$ и для любъх нормальных операторов $N_{1}$ и $N_{2}$ с ограниченной разностъю $N_{1}-N_{2}$

$$
\left\|f\left(N_{1}\right)-f\left(N_{2}\right)\right\| \leqslant c(1-\alpha)^{-1}\|f\|_{\Lambda_{\alpha}}\left\|N_{1}-N_{2}\right\|^{\alpha}
$$

Теорему 3.7.4 можно обобщить на случай произвольных модулей непрерывности и получить аналог теоремы 1.7.3.

ТеОрема 3.7.5. Пусть $0<\alpha<1, p>1$, и пусть $f-$ функция класса Гёльдера $\Lambda_{\alpha}\left(\mathbb{R}^{2}\right)$. Тогда существует положительное число с такое, что

$$
\left\|f\left(N_{1}\right)-f\left(N_{2}\right)\right\|_{S_{p / \alpha}} \leqslant c\|f\|_{\Lambda_{\alpha}}\left\|N_{1}-N_{2}\right\|_{S_{p}}^{\alpha}
$$

для произвольных нормальных операторов $N_{1}$ u $N_{2}$, разность которых входит в класс Шаттена-фон Неймана $\boldsymbol{S}_{p}$.

Мы отсылаем читателя к работе [14], где можно найти доказательства этих утверждений, равно как и других результатов на эту тему.

\section{8. Достаточное условие коммутаторной липшицевости в терминах интегралов Коши}

В этом разделе мы приводим достаточное условие коммутаторной липшицевости, полученное в работе [3].

Пусть $\mathfrak{F}$ - непустое замкнутое подмножество комплексной плоскости $\mathbb{C}$, причём $\mathfrak{F} \neq \mathbb{C}$. Обозначим через $\mathscr{M}(\mathbb{C} \backslash \mathfrak{F})$ множество всех комплексных мер Радона на $\mathbb{C} \backslash \mathfrak{F}$ таких, что

$$
\|\mu\|_{\mathscr{M}(\mathbb{C} \backslash \mathfrak{F})} \stackrel{\text { def }}{=} \sup _{z \in \mathfrak{F}} \int_{\mathbb{C} \backslash \mathfrak{F}} \frac{d|\mu|(\zeta)}{|\zeta-z|^{2}}<+\infty .
$$

Для $\mu \in \mathscr{M}(\mathbb{C} \backslash \mathfrak{F})$ интеграл Коши

$$
\widehat{\mu}(z)=\int_{\mathbb{C} \backslash \mathfrak{F}} \frac{d \mu(\zeta)}{\zeta-z},
$$


вообще говоря, не определён даже при $z \in \mathfrak{F}$, поскольку функция $\zeta \mapsto(\zeta-z)^{-1}$ не обязана быть суммируемой по мере $|\mu|$. С каждой фиксированной точкой $z_{0} \in \mathfrak{F}$ мы связываем модифицированный интеграл Коши следующим образом:

$$
\widehat{\mu}_{z_{0}}(z) \stackrel{\text { def }}{=} \int_{\mathbb{C} \backslash \mathfrak{F}}\left(\frac{1}{\zeta-z}-\frac{1}{\zeta-z_{0}}\right) d \mu(\zeta) .
$$

Из неравенства Коши-Буняковского следует, что величина $\widehat{\mu}_{z_{0}}(z)$ корректно определена при $z \in \mathfrak{F}$ и $\left|\widehat{\mu}_{z_{0}}(z)\right| \leqslant\|\mu\|_{\mathscr{M}(\mathbb{C} \backslash \mathfrak{F})}\left|z-z_{0}\right|$. Kроме того, $\widehat{\mu}_{z_{0}}\left(z_{1}\right)=$ $-\widehat{\mu}_{z_{1}}\left(z_{0}\right)$ и

$$
\left.\left|\widehat{\mu}_{z_{0}}\left(z_{1}\right)-\widehat{\mu}_{z_{0}}\left(z_{2}\right)\right|=\left|\widehat{\mu}_{z_{1}}\left(z_{1}\right)-\widehat{\mu}_{z_{1}}\left(z_{2}\right)\right|=\left|\widehat{\mu}_{z_{1}}\left(z_{2}\right)\right| \leqslant\|\mu\| \mathscr{M}(\mathbb{C} \backslash \mathfrak{F})\right)\left|z_{1}-z_{2}\right|
$$

для любых $z_{1}, z_{2} \in \mathfrak{F}$.

Заметим, что отображение $z \mapsto(\zeta-z)^{-1}$ действует непрерывно из множества $\mathfrak{F}$ в гильбертово пространство $L^{2}(|\mu|)$, наделённое слабой топологией. Это обстоятельство позволяет легко проверить, что функция $\widehat{\mu}_{z_{0}}(z)$ дифференцируема, как функция комплексной переменной, в каждой неизолированной точке множества $\mathfrak{F}$. В частности, $\widehat{\mu}_{z_{0}}(z)$ аналитична во внутренности множества $\mathfrak{F}$.

Обозначим символом $\widehat{\mathscr{M}}(\mathfrak{F})$ множество всех функций $f$ на $\mathfrak{F}$, представимых в виде $f=c+\widehat{\mu}_{z_{0}}$, где $c-$ константа. Положим

$$
\|f\|_{\widehat{\mathscr{M}(\mathfrak{F})}} \stackrel{\text { def }}{=} \inf \left\{\|\mu\|_{\mathscr{M}(\mathbb{C} \backslash \mathfrak{F})}: \mu \in \mathscr{M}(\mathbb{C} \backslash \mathfrak{F}), f-\widehat{\mu}_{z_{0}}=\text { const на } \mathfrak{F}\right\} .
$$

Легко видеть, что определение пространства $\widehat{\mathscr{M}}(\mathfrak{F})$ и полунормы $\|f\|_{\widehat{\mathscr{M}}(\mathfrak{F})}$ не зависит от выбора точки $z_{0} \in \mathfrak{F}$.

Tеорема 3.8.1. Пусть $\mathfrak{F}$ - собственное замкнутое подмножество комплексной плоскости $\mathbb{C}$. Тогда $\widehat{\mathscr{M}(\mathfrak{F}) \subset \mathrm{CL}(\mathfrak{F})} u\|f\|_{\mathrm{CL}(\mathfrak{F})} \leqslant\|f\|_{\widehat{\mathscr{M}}(\mathfrak{F})}$ для всех $f$ из $\widehat{\mathscr{M}}(\mathfrak{F})$.

ДоказАтельство. Пусть $\mu \in \mathscr{M}(\mathbb{C} \backslash \mathfrak{F})$ и $f=\widehat{\mu}_{z_{0}}$. Рассмотрим разделённую разность

$$
\frac{f(z)-f(w)}{z-w}=\frac{1}{z-w} \int_{\mathbb{C} \backslash \mathfrak{F}}\left(\frac{1}{\zeta-z}-\frac{1}{\zeta-w}\right) d \mu(\zeta)=\int_{\mathbb{C} \backslash \mathfrak{F}} \frac{d \mu(\zeta)}{(\zeta-z)(\zeta-w)} .
$$

Неравенство (3.8.1) означает, что эта разделённая разность удовлетворяет условию (d) теоремы 2.3.1. Стало быть, она является мультипликатором Шура для любых спектральных борелевских мер на $\mathfrak{F}$. Причём её мультипликаторная норма не превосходит $\|\mu\|_{\mathscr{M}(\mathbb{C} \backslash \mathfrak{F})}$. Остаётся сослаться на теорему 3.3.2.

Пусть $\widehat{\mathscr{M}}_{\infty}(\mathfrak{F})$ обозначает пространство функций вида $f+a z$, где $f \in \widehat{\mathscr{M}}(\mathfrak{F})$, $a \in \mathbb{C}$. Легко видеть, что линейная функция $a z$ принадлежит пространству $\widehat{\mathscr{M}}(\mathfrak{F})$, если множество $\mathfrak{F}$ компактно. Таким образом, $\widehat{\mathscr{M}}_{\infty}(\mathfrak{F})=\widehat{\mathscr{M}}(\mathfrak{F})$ для компактных $\mathfrak{F}$. В случае неограниченного множества $\mathfrak{F}$ нетрудно проверить, что $f^{\prime}(\infty)=0$ для любой функции $f \in \widehat{\mathscr{M}}(\mathfrak{F})$. Таким образом, $\widehat{\mathscr{M}}_{\infty}(\mathfrak{F}) \neq \widehat{\mathscr{M}}(\mathfrak{F})$ для некомпактных множеств $\mathfrak{F}$. Из теоремы 3.8.1 следует, что $\widehat{\mathscr{M}}_{\infty}(\mathfrak{F}) \subset \mathrm{CL}(\mathfrak{F})$.

Авторам неизвестно, имеет ли место равенство $\widehat{\mathscr{M}}_{\infty}(\mathfrak{F})=\mathrm{CL}(\mathfrak{F})$ даже для таких простых множеств $\mathfrak{F}$, как окружность или прямая. 


\section{9. Коммутаторно липшицевы функции в круге и в полуплоскости}

Мы рассмотрим здесь пространства коммутаторно липшицевых функций в единичном круге $\mathbb{D}$ и в верхней полуплоскости $\mathbb{C}_{+} \stackrel{\text { def }}{=}\{\zeta \in \mathbb{C}: \operatorname{Re} \zeta>0\}$. В частности, мы изложим результаты работы Э. Киссина и В. С. Шульмана [39] и их аналоги для верхней полуплоскости.

Пусть $\mathrm{C}_{\mathrm{A}}$ обозначает диск-алгебру, т. е. пространство всех функций $f$, аналитических в открытом круге $\mathbb{D}$ и непрерывных в его замыкании. В работе [39] доказано, что $\mathrm{CL}(\mathbb{D})=\left\{f \in \mathrm{C}_{\mathrm{A}}: f \in \mathrm{OL}(\mathbb{T})\right\}$. Следующая теорема показывает, что при этом имеет место равенство норм.

TeOpema 3.9.1. Пусть $f \in \mathrm{CL}(\mathbb{D})$. Тогда $f \in \mathrm{C}_{\mathrm{A}} u\|f\|_{\mathrm{CL}(\mathbb{D})}=\|f\|_{\mathrm{CL}(\mathbb{T})}=$ $\|f\|_{\mathrm{OL}(\mathbb{T})}$. Если $f \in \mathrm{C}_{\mathrm{A}}$, то $f \in \mathrm{CL}(\mathbb{D})$ в том и только том случае, когда $f \in \mathrm{OL}(\mathbb{T})$.

ДокАЗАтельство. Равенство $\|f\|_{\mathrm{CL}(\mathbb{T})}=\|f\|_{\mathrm{OL}(\mathbb{T})}$ следует из теоремы 3.1.10. Неравенство $\|f\|_{\mathrm{CL}(\mathbb{T})} \leqslant\|f\|_{\mathrm{CL}(\mathbb{D})}$ очевидно. Остаётся доказать, что $\|f\|_{\mathrm{CL}(\mathbb{D})} \leqslant$ $\|f\|_{\mathrm{CL}(\mathbb{T})}$. Можно считать, что $\|f\|_{\mathrm{CL}(\mathbb{T})}=1$. Тогда $\|\mathfrak{D} f\|_{\mathfrak{M}(\mathbb{T} \times \mathbb{T})}=1$ в силу теоремы 3.3.6. Применим теперь теорему 2.2.3. Тогда мы получим два семейства $\left\{u_{\zeta}\right\}_{\zeta \in \mathbb{T}}$ и $\left\{v_{\tau}\right\}_{\tau \in \mathbb{T}}$ в некотором гильбертовом пространстве $\mathscr{H}$, непрерывно зависящие в слабой топологии от параметров и такие, что $\left\|u_{\zeta}\right\| \leqslant 1,\left\|v_{\tau}\right\| \leqslant 1$ и $(\mathfrak{D} f)(\zeta, \tau)=\left(u_{\zeta}, v_{\tau}\right)$ при всех $\zeta, \tau \in \mathbb{T}$. Рассмотрим гармонические продолжения функций $\zeta \mapsto u_{\zeta}$ и $\tau \mapsto v_{\tau}$ в единичный круг, положив

$$
u_{z} \stackrel{\text { def }}{=} \int_{\mathbb{T}} \frac{1-|z|^{2}}{|z-\zeta|^{2}} u_{\zeta} d \boldsymbol{m}(\zeta) \quad \text { и } \quad v_{w} \stackrel{\text { def }}{=} \int_{\mathbb{T}} \frac{1-|w|^{2}}{|w-\tau|^{2}} v_{\tau} d \boldsymbol{m}(\tau)
$$

при $z, w \in \mathbb{D}$. Интегралы понимаются как интегралы $\mathscr{H}$-значных функций, непрерывных в слабой топологии. Применяя интеграл Пуассона по переменной $\zeta$ к обеим частям равенства $(\mathfrak{D} f)(\zeta, \tau)=\left(u_{\zeta}, v_{\tau}\right)$, получаем $(\mathfrak{D} f)(z, \tau)=$ $\left(u_{z}, v_{\tau}\right)$ при всех $z \in \operatorname{clos} \mathbb{D}$ и $\tau \in \mathbb{T}$. Применяя теперь интеграл Пуассона к последнему равенству, получаем: $(\mathfrak{D} f)(z, w)=\left(u_{z}, v_{w}\right)$ при всех $z \in \operatorname{clos} \mathbb{D}$ и $w \in \operatorname{clos} \mathbb{D}$. Теперь ясно, что

$$
\begin{aligned}
\|f\|_{\mathrm{CL}(\mathbb{D})} & =\|\mathfrak{D} f\|_{\mathfrak{M}(\cos \mathbb{D} \times \operatorname{clos} \mathbb{D})} \leqslant \sup _{z \in \operatorname{clos} \mathbb{D}}\left\|u_{z}\right\| \sup _{w \in \operatorname{clos} \mathbb{D}}\left\|v_{w}\right\| \\
& =\sup _{\zeta \in \mathbb{T}}\left\|u_{\zeta}\right\| \sup _{\tau \in \mathbb{T}}\left\|v_{\tau}\right\|=1 .
\end{aligned}
$$

Теорема доказана.

Приведём теперь аналог теоремы 2.2.4 для функций в единичном круге.

Tеорема 3.9.2. Пусть $f \in \mathrm{CL}(\mathbb{D})$. Тогда в диск-алгебре $\mathrm{C}_{\mathrm{A}}$ существуют последовательности $\left\{\varphi_{n}\right\}_{n \geqslant 1} u\left\{\psi_{n}\right\}_{n \geqslant 1}$ такие, ито

$$
\begin{gathered}
\left(\sup _{z \in \mathbb{D}} \sum_{n=1}^{\infty}\left|\varphi_{n}(z)\right|^{2}\right)\left(\sup _{w \in \mathbb{D}} \sum_{n=1}^{\infty}\left|\psi_{n}(w)\right|^{2}\right)=\|f\|_{\mathrm{CL}(\mathbb{D})}^{2}, \\
(\mathfrak{D} f)(z, w)=\sum_{n=1}^{\infty} \varphi_{n}(z) \psi_{n}(w),
\end{gathered}
$$

при этом все ряды сходятся равномерно, когда переменные $z$ u $w$ пробегают компактное подмножество открытого единичного круга. 
ДоказАтеЛьство. Можно считать, что $\|f\|_{\mathrm{CL}(\mathbb{D})}=1$. Вместо первого равенства достаточно доказать неравенство $\leqslant$, ибо неравенство $\geqslant$ вытекает из теорем 3.3.6 и 2.2.1. Пусть $\mathscr{H}, u_{z}$ и $v_{w}$ обозначают то же, что в доказательстве теоремы 3.9.1. Рассмотрим ортонормированный базис $\left\{e_{n}\right\}_{n=1}^{\infty}$ в $\mathscr{H}$. Положим $\varphi_{n}(z) \stackrel{\text { def }}{=}\left(u_{z}, e_{n}\right)$ и $\psi_{n}(w) \stackrel{\text { def }}{=}\left(e_{n}, v_{w}\right)$ для всех $n \geqslant 1$. Докажем, что $\varphi_{n} \in \mathrm{C}_{\mathrm{A}}$ и $\psi_{n} \in \mathrm{C}_{\mathrm{A}}$. Обозначим символом $X$ множество векторов $e \in \mathscr{H}$ таких, что $\left(u_{z}, e\right) \in \mathrm{C}_{\mathrm{A}}$. Ясно, что $X$ - замкнутое подпространство пространства $\mathscr{H}$. Заметим, что $v_{\tau} \in X$ для всех $\tau \in \mathbb{T}$, поскольку $(\mathfrak{D} f)(\cdot, \tau) \in \mathrm{C}_{\mathrm{A}}$ при всех $\tau \in \mathbb{T}$. Следовательно, $X=\mathscr{H}$, ибо линейная оболочка семейства $\left\{v_{\tau}\right\}_{\tau \in \mathbb{T}}$ плотна в $\mathscr{H}$. Таким образом, $\left(u_{z}, e\right) \in \mathrm{C}_{\mathrm{A}}$ для всех $e \in \mathscr{H}$. Аналогично доказывается, что $\left(e, v_{w}\right) \in \mathrm{C}_{\mathrm{A}}$ для всех $e \in \mathscr{H}$. Остаётся доказать утверждение о равномерной сходимости на компактах. Заметим, что

$$
\left|\sum_{n=N}^{\infty} \varphi_{n}(z) \psi_{n}(w)\right| \leqslant\left(\sum_{n=N}^{\infty}\left|\varphi_{n}(z)\right|^{2}\right)^{1 / 2}\left(\sum_{n=N}^{\infty}\left|\psi_{n}(w)\right|^{2}\right)^{1 / 2} .
$$

Таким образом, достаточно доказать равномерную сходимость на компактных подмножествах круга $\mathbb{D}$ рядов $\sum_{n=1}^{\infty}\left|\varphi_{n}(z)\right|^{2}$ и $\sum_{n=1}^{\infty}\left|\psi_{n}(z)\right|^{2}$. Это вытекает из следующей элементарной леммы.

Лемма 3.9.3. Пусть $\left\{h_{k}\right\}_{k=1}^{\infty}-$ последовательность аналитических в единичном круге $\mathbb{D}$ функций. Предположим, что функиия $\sum_{k=1}^{\infty}\left|h_{k}(z)\right|$ ограничена в $\mathbb{D}$. Тогда ряд $\sum_{k=1}^{\infty}\left|h_{k}(z)\right|$ сходится равномерно на компактных подмножествах открытого единичного круга.

Обозначим через $(\mathrm{OL})_{+}(\mathbb{T})$ пространство функций $f$ из $\mathrm{OL}(\mathbb{T})$, допускающих аналитическое продолжение в единичный круг $\mathbb{D}$, непрерывное вплоть до границы. Из теоремы 3.3 .3 следует, что каждая функция $f \in \mathrm{CL}(\mathbb{D})$ аналитична в $\mathbb{D}$. Таким образом, из теоремы 3.9 .1 вытекает следующий результат работы [39].

ТеОрема 3.9.4. Оператор сужения $f \mapsto f \mid \mathbb{T}$ является линейной изометрией пространства $\mathrm{CL}(\mathbb{D})$ на пространство $(\mathrm{OL})_{+}(\mathbb{T})$.

Аналогичные результаты имеют место и для пространства $\mathrm{CL}\left(\mathbb{C}_{+}\right)$.

Теорема 3.9.5. Пусть $f$ - непрерывная функиия в замкнутой верхней полуплоскости сlos $\mathbb{C}_{+}$. Предположим, что функция $f$ аналитична в открытой полуплоскости $\mathbb{C}_{+}$. Тогда $\|f\|_{\mathrm{CL}\left(\mathbb{C}_{+}\right)}=\|f\|_{\mathrm{CL}(\mathbb{R})}=\|f\|_{\mathrm{OL}(\mathbb{R})} \cdot$. В частности, $f \in \mathrm{CL}\left(\mathbb{C}_{+}\right)$в том и только том случае, когда $f \in \mathrm{OL}(\mathbb{R})$.

Обозначим через $\mathrm{C}_{\mathrm{A}}\left(\mathbb{C}_{+}\right)$множество всех аналитических в $\mathbb{C}_{+}$функций, непрерывных вплоть до границы и имеющих конечный предел в бесконечности. 
Tеорема 3.9.6. Пусть $f \in \mathrm{CL}\left(\mathbb{C}_{+}\right)$. Тогда в пространстве $\mathrm{C}_{\mathrm{A}}\left(\mathbb{C}_{+}\right)$существуют последователъности $\left\{\varphi_{n}\right\}_{n=1}^{\infty} u\left\{\psi_{n}\right\}_{n=1}^{\infty}$ такие, что

$$
\begin{gathered}
\left(\sup _{z \in \mathbb{C}_{+}} \sum_{n=1}^{\infty}\left|\varphi_{n}(z)\right|^{2}\right)\left(\sup _{w \in \mathbb{C}_{+}} \sum_{n=1}^{\infty}\left|\psi_{n}(w)\right|^{2}\right)=\|f\|_{\mathrm{CL}\left(\mathbb{C}_{+}\right)}^{2}, \\
(\mathfrak{D} f)(z, w)=\sum_{n=1}^{\infty} \varphi_{n}(z) \psi_{n}(w)
\end{gathered}
$$

При этом все ряды сходятся равномерно, когда переменные $z$ u $w$ пробегают компактное подмножество открытой верхней полуплоскости.

Мы опускаем здесь доказательства теорем 3.9.5 и 3.9.6. Они аналогичны доказательствам соответствующих результатов для функций в единичном круге.

Имеет место также следующий аналог для прямой теоремы 3.9.4.

Теорема 3.9.7. Оператор сужения $f \mapsto f \mid \mathbb{R}$ является линейной изометрией пространства $\mathrm{CL}\left(\mathbb{C}_{+}\right)$на пространство $(\mathrm{OL})_{+}(\mathbb{R})$.

Отметим, что в статье [5] получен следующий результат, по существу содержащий в себе как теорему 3.9.1, так и теорему 3.9.5.

ТеОРема 3.9.8. Пусть $\mathfrak{F}_{0}$ и $\mathfrak{F}$ - непустые совериенные подмножества комплексной плоскости $\mathbb{C}$ такие, что $\mathfrak{F}_{0} \subset \mathfrak{F} u$ множество $\Omega \stackrel{\text { def }}{=} \mathfrak{F} \backslash \mathfrak{F}_{0}$ открыто. Предположим, что функиия $f_{0} \in \mathrm{CL}\left(\mathfrak{F}_{0}\right)$ допускает непрерывное продолжение $f$ на множество $\mathfrak{F}$ такое, что функиия $f$ аналитична на $\Omega$ $u\left|f(z) z^{-2}\right| \rightarrow 0$ при $z \rightarrow \infty$ в каждой неограниченной ${ }^{3}$ компоненте связности множества $\Omega$. Тогда $f \in \mathrm{CL}(\mathfrak{F}) u\|f\|_{\mathrm{CL}(\mathfrak{F})}=\left\|f_{0}\right\|_{\mathrm{CL}\left(\mathfrak{F}_{0}\right)}$.

Авторам неизвестен ответ на следующий вопрос. Пусть $f$ - непрерывная функция в замкнутом единичном круге, гармоническая внутри круга. Предположим, что $f \in \mathrm{OL}(\mathbb{T})$. Вытекает ли отсюда, что $f \in \mathrm{OL}(\mathbb{D})$ ? Аналогичный вопрос можно поставить для полуплоскости и для других областей.

Напомним, что если $T$ - сжатие в гильбертовом пространстве $\mathscr{H}$, то по теореме Сёкефальви-Надя (см. [72; гл. I, § 5]) существует его унитарная дилатаиия, т. е. такой унитарный оператор $U$ в гильбертовом пространстве $\mathscr{K}, \mathscr{H} \subset \mathscr{K}$, что $T^{n}=P_{\mathscr{H}} U^{n} \mid \mathscr{H}, n \geqslant 0$. Дилатацию всегда можно выбрать минимальной. При этом можно определить линейное и мультипликативное функциональное исчисление: $\varphi \mapsto \varphi(T) \stackrel{\text { def }}{=} P_{\mathscr{H}} \varphi(U) \mid \mathscr{H}, \varphi \in \mathrm{C}_{\mathrm{A}}$. Полуспектральная мера $\mathcal{E}_{T}$ сжатия $T$ определяется равенством $\mathcal{E}_{T}(\Delta) \stackrel{\text { def }}{=} P_{\mathscr{H}} E_{U}(\Delta) \mid h$, где $E_{U}$ - спектральная мера оператора $U$, а $\Delta$ - борелевское подмножество окружности $\mathbb{T}$. Легко видеть, что

$$
\varphi(T)=\int_{\mathbb{T}} \varphi(\zeta) d \mathcal{E}(\zeta), \quad \varphi \in \mathrm{C}_{\mathrm{A}} .
$$

\footnotetext{
${ }^{3}$ Последнее условие выполняется автоматически, если множество $\Omega$ ограничено.
} 
Tеорема 3.9.9. Пусть $f \in \mathrm{CL}(\mathbb{D})$, и пусть $T_{1}$ и $T_{2}-$ сжатия в гильбертовом пространстве $\mathscr{H}$, а $R \in \mathcal{B}(\mathscr{H})$. Тогда

$$
f\left(T_{1}\right) R-R f\left(T_{2}\right)=\int_{\mathbb{T}} \int_{\mathbb{T}}(\mathfrak{D} f)(\zeta, \tau) d \mathcal{E}_{1}(\zeta)\left(T_{1} R-R T_{2}\right) d \mathcal{E}_{2}(\tau),
$$

где $\mathcal{E}_{1}$ и $\mathcal{E}_{2}$ - полуспектральные меры сжатий $T_{1}$ и $T_{2}$, и справедливо неравенcтво

$$
\left\|f\left(T_{1}\right) R-R f\left(T_{2}\right)\right\| \leqslant\|f\|_{\mathrm{CL}(\mathbb{D})}\left\|T_{1} R-R T_{2}\right\| .
$$

ДоказАтельство. Пусть $\left\{\varphi_{n}\right\}_{n \geqslant 1}$ и $\left\{\psi_{n}\right\}_{n \geqslant 1}-$ последовательности функций в диск-алгебре, удовлетворяющие заключению теоремы 3.9.2. Ввиду формулы (2.3.7)

$$
\begin{aligned}
\int_{\mathbb{T}} \int_{\mathbb{T}} & (\mathfrak{D} f)(\zeta, \tau) d \mathcal{E}_{1}(\zeta)\left(T_{1} R-R T_{2}\right) d \mathcal{E}_{2}(\tau)=\sum_{n \geqslant 1} \varphi_{n}\left(T_{1}\right)\left(T_{1} R-R T_{2}\right) \psi_{n}\left(T_{2}\right) \\
= & \sum_{n \geqslant 1} T_{1} \varphi_{n}\left(T_{1}\right) R \psi_{n}\left(T_{2}\right)-\sum_{n \geqslant 1} \varphi_{n}\left(T_{1}\right) R \psi_{n}\left(T_{2}\right) T_{2} \\
= & \int_{\mathbb{T}} \int_{\mathbb{T}} \zeta(\mathfrak{D} f)(\zeta, \tau) d \mathcal{E}_{1}(\zeta) R d \mathcal{E}_{2}(\tau)-\int_{\mathbb{T}} \int_{\mathbb{T}} \tau(\mathfrak{D} f)(\zeta, \tau) d \mathcal{E}_{1}(\zeta) R d \mathcal{E}_{2}(\tau) \\
= & \int_{\mathbb{T}} \int_{\mathbb{T}}(f(\zeta)-f(\tau)) d \mathcal{E}_{1}(\zeta) R d \mathcal{E}_{2}(\tau)=f\left(T_{1}\right) R-R f\left(T_{2}\right),
\end{aligned}
$$

что доказывает формулу (3.9.1), которая немедленно влечёт неравенство (3.9.2). Теорема доказана.

Неравенство (3.9.2) было доказано Э. Киссиным и В. С. Шульманом в [39] другим методом. Доказательство, приведённое здесь, аналогично доказательству теоремы 4.1 в работе [62] (см. также [57]). В случае $f \in B_{\infty, 1}^{1}(\mathbb{T}) \cap \mathrm{C}_{\mathrm{A}}$ и $R=I$ теорема 3.9 .9 была доказана в работе [57].

Аналогичное утверждение можно доказать и для диссипативных операторов (см. [12] по поводу возмущений функций диссипативных операторов).

\subsection{0. Операторно липшицевы функции и дробно-линейные преобразования}

Пусть $\operatorname{Aut}(\widehat{\mathbb{C}})$ обозначает группу Мёбиуса дробно-линейных преобразований расширенной комплексной плоскости $\widehat{\mathbb{C}} \stackrel{\text { def }}{=} \mathbb{C} \cup\{\infty\}$. Другими словами,

$$
\operatorname{Aut}(\widehat{\mathbb{C}})=\left\{\varphi: \varphi(z)=\frac{a z+b}{c z+d}, a, b, c, d \in \mathbb{C}, a d-b c \neq 0\right\} .
$$

Множество линейных преобразований комплексной плоскости обозначим чеpeз $\operatorname{Aut}(\mathbb{C})$, т. е.

$$
\operatorname{Aut}(\mathbb{C})=\{\varphi \in \operatorname{Aut}(\widehat{\mathbb{C}}): \varphi(\infty)=\infty\}=\{\varphi: \varphi(z)=a z+b, a, b \in \mathbb{C}, a \neq 0\} .
$$

Пусть $\widehat{\mathbb{R}}$ обозначает расширенную вещественную прямую: $\widehat{\mathbb{R}} \stackrel{\text { def }}{=} \mathbb{R} \cup\{\infty\}$. Положим

$$
\operatorname{Aut}(\widehat{\mathbb{R}}) \stackrel{\text { def }}{=}\{\varphi \in \operatorname{Aut}(\widehat{\mathbb{C}}): \varphi(\widehat{\mathbb{R}})=\widehat{\mathbb{R}}\} \quad \text { и } \quad \operatorname{Aut}(\mathbb{R}) \stackrel{\text { def }}{=}\{\varphi \in \operatorname{Aut}(\mathbb{C}): \varphi(\mathbb{R})=\mathbb{R}\} .
$$


С каждым дробно-линейным преобразованием $\varphi$ и с каждой функцией $f$, заданной на замкнутом множестве $\mathfrak{F}, \mathfrak{F} \subset \mathbb{C}$, мы связываем функцию $\mathcal{Q}_{\varphi} f$, определённую на замкнутом множестве $\mathfrak{F}_{\varphi} \stackrel{\text { def }}{=} \mathbb{C} \cap \varphi^{-1}(\mathfrak{F} \cup\{\infty\})$ формулой

$$
\left(\mathcal{Q}_{\varphi} f\right)(z) \stackrel{\text { def }}{=} \begin{cases}\frac{f(\varphi(z))}{\varphi^{\prime}(z)}, & \text { если } z \in \mathbb{C}, \varphi(z) \in \mathfrak{F} \text { и } \varphi(z) \neq \infty, \\ 0, & \text { если } z \in \mathbb{C} \text { и } \varphi(z)=\infty .\end{cases}
$$

Легко видеть, что если $\varphi \in \operatorname{Aut}(\mathbb{C})$, то имеют место следующие равенства: $\mathfrak{F}_{\varphi}=\varphi^{-1}(\mathfrak{F}), \mathcal{Q}_{\varphi} f=\left(\varphi^{\prime}(0)\right)^{-1}(f \circ \varphi), \mathcal{Q}_{\varphi}(\mathrm{OL}(\mathfrak{F}))=\operatorname{OL}\left(\mathfrak{F}_{\varphi}\right), \mathcal{Q}_{\varphi}(\mathrm{CL}(\mathfrak{F}))=$ $\mathrm{CL}\left(\mathfrak{F}_{\varphi}\right),\left\|\mathcal{Q}_{\varphi} f\right\|_{\mathrm{OL}\left(\mathfrak{F}_{\varphi}\right)}=\|f\|_{\mathrm{OL}(\mathfrak{F})}$ для всех $f$ из $\mathrm{OL}(\mathfrak{F})$ и $\left\|\mathcal{Q}_{\varphi} f\right\|_{\mathrm{CL}(\mathfrak{F} \varphi)}=\|f\|_{\mathrm{CL}(\mathfrak{F})}$ для всех $f$ из $\mathrm{CL}(\mathfrak{F})$. Поэтому в дальнейшем нас будет в основном интересовать случай, когда $\varphi \notin \operatorname{Aut}(\mathbb{C})$. Заметим, что если $\mathfrak{F}=\mathbb{C}$, то $\mathfrak{F}_{\varphi}=\mathbb{C}$ при всех $\varphi$ из $\operatorname{Aut}(\widehat{\mathbb{C}})$. Если же $\mathfrak{F}=\mathbb{R}$, то $\mathfrak{F}_{\varphi}=\mathbb{R}$ при всех $\varphi$ из $\operatorname{Aut}(\widehat{\mathbb{R}})$.

Пусть $a \in \mathfrak{F}$, где $\mathfrak{F}$ - замкнутое подмножество комплексной плоскости $\mathbb{C}$. Положим

$$
\mathrm{OL}_{a}(\mathfrak{F}) \stackrel{\text { def }}{=}\{f \in \mathrm{OL}(\mathfrak{F}): f(a)=0\} \quad \text { и } \quad \mathrm{CL}_{a}(\mathfrak{F}) \stackrel{\text { def }}{=}\{f \in \mathrm{CL}(\mathfrak{F}): f(a)=0\} .
$$

Ясно, что $\mathrm{OL}_{a}(\mathfrak{F})$ и $\mathrm{CL}_{a}(\mathfrak{F})$ - банаховы пространства.

TеОРема 3.10.1. Пусть $\mathfrak{F}$ - замкнутое подмножество в $\mathbb{C}, a \in \mathfrak{F}$, и пусть $\varphi$ - автоморфизм из Aut $(\widehat{\mathbb{C}})$ такой, что $a \stackrel{\text { def }}{=} \varphi(\infty)$. Тогда $\mathcal{Q}_{\varphi}\left(\mathrm{OL}_{a}(\mathfrak{F})\right) \subset$ $\mathrm{OL}\left(\mathfrak{F}_{\varphi}\right) u$

$$
\left\|\mathcal{Q}_{\varphi} f\right\|_{\mathrm{OL}\left(\mathfrak{F}_{\varphi}\right)} \leqslant 3\|f\|_{\mathrm{OL}(\mathfrak{F})} \quad \text { для всех } f \text { из } \mathrm{OL}_{a}(\mathfrak{F})
$$

ДокАЗАтельство. Рассмотрим сначала случай $\varphi(z)=\phi(z) \stackrel{\text { def }}{=} z^{-1}$. Тогда $a=0$ и нам нужно доказать, что $\mathcal{Q}_{\phi}\left(\mathrm{OL}_{0}(\mathfrak{F})\right) \subset \mathrm{OL}\left(\mathfrak{F}_{\varphi}\right)$ и $\left\|\mathcal{Q}_{\phi} f\right\|_{\mathrm{OL}\left(\mathfrak{F}_{\varphi}\right)} \leqslant$ $3\|f\|_{\mathrm{OL}(\mathfrak{F})}$. Пусть $f \in \mathrm{OL}_{0}(\mathfrak{F})$. Можно считать, что $\|f\|_{\mathrm{OL}(\mathfrak{F})}=1$. Тогда всё сводится к неравенству

$$
\left\|\left(\mathcal{Q}_{\varphi} f\right)(N) R-R\left(\mathcal{Q}_{\varphi} f\right)(N)\right\| \leqslant 3 \max \left\{\|N R-R N\|,\left\|N^{*} R-R N^{*}\right\|\right\}
$$

для любых ограниченных операторов $N$ и $R$ таких, что оператор $N$ нормален и $\sigma(N) \subset \mathfrak{F}_{\phi}$. Рассмотрим функцию $h$ такую, что $h(z)=z f\left(z^{-1}\right)$ при $z \neq 0$ и $f(0)=0$. Легко видеть, что $\sup |h| \leqslant\|f\|_{\operatorname{Lip}(\mathfrak{F})} \leqslant\|f\|_{\mathrm{OL}(\mathbb{R})}=1$, поскольку $f(0)=0$. Заметим, что $\left(\mathcal{Q}_{\phi} f\right)(N)=-N h(N)$. Таким образом, нам нужно доказать, что

$$
\|N h(N) R-R N h(N)\| \leqslant 3 \max \left\{\|N R-R N\|,\left\|N^{*} R-R N^{*}\right\|\right\} .
$$

Мы воспользуемся следующим элементарным тождеством:

$$
\begin{aligned}
N h(N) R-R N h(N)=h & (N)(N R-R N) \\
& +h(N) R N-N R h(N)+(N R-R N) h(N) .
\end{aligned}
$$

Заметим, что

$$
\|h(N)(N R-R N)\| \leqslant\|N R-R N\| \leqslant \max \left\{\|N R-R N\|,\left\|N^{*} R-R N^{*}\right\|\right\} .
$$

Аналогично оценивается и норма оператора $(N R-R N) h(N)$. 
Остаётся доказать, что

$$
\|h(N) R N-N R h(N)\| \leqslant \max \left\{\|N R-R N\|,\left\|N^{*} R-R N^{*}\right\|\right\} .
$$

Если оператор $N$ обратим, то

$$
\begin{aligned}
\|h(N) R N-N R h(N)\| & =\left\|f\left(N^{-1}\right) N R N-N R N f\left(N^{-1}\right)\right\| \\
& \leqslant \max \left\{\|R N-N R\|,\left\|\left(N^{*}\right)^{-1} N R N-N R N\left(N^{*}\right)^{-1}\right\|\right\} \\
& =\max \left\{\|N R-R N\|,\left\|\left(N^{*}\right)^{-1} N\left(R N^{*}-N^{*} R\right) N\left(N^{*}\right)^{-1}\right\|\right\} \\
& =\max \left\{\|N R-R N\|,\left\|N^{*} R-R N^{*}\right\|\right\} .
\end{aligned}
$$

Если 0 - предельная точка множества $\mathfrak{F}_{\phi}$ (т. е. множество $\mathfrak{F}$ неограничено), то на этом доказательство можно закончить, ибо в этом случае каждый нормальный оператор $N$ со спектром в $\mathfrak{F}_{\phi}$ приближается с любой точностью нормальным оператором $M$ таким, что $M N=N M$ и $\sigma(M) \subset \mathfrak{F}_{\varphi} \backslash\{0\}$. Это следует из леммы 3.1.12.

Пусть теперь 0 - изолированная точка множества $\mathfrak{F}_{\phi}$. Рассмотрим необратимый нормальный оператор $N$ со спектром $\mathfrak{F}_{\phi}$. Тогда оператор $N$ представим в виде $N=\mathbf{0} \oplus N_{0}$, где $N_{0}$ - обратимый нормальный оператор. Заметим, что $\mathcal{Q}_{\phi}(N)=\mathbf{0} \oplus N_{0}^{2} f\left(N_{0}^{-1}\right)$. Пусть $P$ обозначает ортогональный проектор на подпространство, в котором действует оператор $N_{0}$. Легко видеть, что

$$
\begin{aligned}
& \|h(N) R N-N R h(N)\|=\|P(h(N) R N-N R h(N)) P\| \\
& \quad=\|h(N) P R P N-N P R P h(N)\|=\left\|h\left(N_{0}\right)(P R P) N_{0}-N_{0}(P R P) h\left(N_{0}\right)\right\| \\
& \quad \leqslant \max \left\{\left\|N_{0}(P R P)-(P R P) N_{0}\right\|,\left\|N_{0}^{*}(P R P)-(P R P) N_{0}^{*}\right\|\right\} \\
& \quad \leqslant \max \left\{\|N R-R N\|,\left\|N^{*} R-R N^{*}\right\|\right\} .
\end{aligned}
$$

Переходим к общему случаю. Положим $b=\varphi^{-1}(\infty)$. Ясно, что $\varphi(z)=$ $a+c \phi(z-b)$, где $c \in \mathbb{C} \backslash\{0\}$. Таким образом, всё сводится к случаю, когда $a=$ $b=0$, т. е. $\varphi=c \phi$, поскольку параллельные переносы сохраняют операторно липшицеву норму. Наконец, случай $\varphi=c \phi$ легко сводится к разобранному случаю $\varphi=\phi$. Теорема доказана.

ПримеР. Пусть $\varphi(z)=z^{-1}, \mathfrak{F}=\mathbb{C}, f=\bar{z}$. Тогда $f \in \mathrm{OL}_{0}(\mathbb{C})$ и $\|f\|_{\mathrm{OL}(\mathbb{C})}=1$. Кроме того, $\left(\mathcal{Q}_{\varphi} f\right)(z)=-\bar{z}^{-1} z^{2}$ и

$$
3=\left\|\mathcal{Q}_{\varphi} f\right\|_{\operatorname{Lip}(\mathbb{T})} \leqslant\left\|\mathcal{Q}_{\varphi} f\right\|_{\operatorname{Lip}(\mathbb{C})} \leqslant\left\|\mathcal{Q}_{\varphi} f\right\|_{\mathrm{OL}(\mathbb{C})} \leqslant 3\|f\|_{\mathrm{OL}(\mathbb{C})}=3
$$

Этот пример показывает, что $\left\|\mathcal{Q}_{\varphi} f\right\|_{\mathrm{OL}(\mathbb{C})}=\left\|\mathcal{Q}_{\varphi} f\right\|_{\operatorname{Lip}(\mathbb{C})}=3$ и константа 3 в теореме 3.10.1 неулучшаема.

Из теоремы 3.10.1 легко вывести следующее утверждение.

TeOpema 3.10.2. Пусть $\varphi \in \operatorname{Aut}(\widehat{\mathbb{C}}), a=\varphi(\infty) u b=\varphi^{-1}(\infty)$. Пусть $\mathfrak{F}$ - замкнутое множество в $\mathbb{C}$, содержащее точку а. Тогда $\mathcal{Q}_{\varphi}\left(\mathrm{OL}_{a}(\mathfrak{F})\right)=$ $\mathrm{OL}_{b}\left(\mathfrak{F}_{\varphi}\right) u$

$$
\frac{1}{3}\|f\|_{\mathrm{OL}(\mathfrak{F})} \leqslant\left\|\mathcal{Q}_{\varphi} f\right\|_{\mathrm{OL}\left(\mathfrak{F}_{\varphi}\right)} \leqslant 3\|f\|_{\mathrm{OL}(\mathfrak{F})} \quad \text { для всех } f \text { из } \mathrm{OL}_{a}(\mathfrak{F}) .
$$


ДоказАтельство. Заметим, что $\left(\mathcal{Q}_{\varphi}\left(\mathrm{OL}_{a}(\mathfrak{F})\right)\right)(b)=0$. Таким образом, из теоремы 3.10 .1 следует, что $\mathcal{Q}_{\varphi}\left(\mathrm{OL}_{a}(\mathfrak{F})\right) \subset \mathrm{OL}_{b}\left(\mathfrak{F}_{\varphi}\right)$ и $\left\|\mathcal{Q}_{\varphi} f\right\|_{\mathrm{OL}\left(\mathfrak{F}_{\varphi}\right)} \leqslant 3\|f\|_{\mathrm{OL}(\mathfrak{F})}$. Чтобы доказать, что $\mathcal{Q}_{\varphi}\left(\mathrm{OL}_{a}(\mathfrak{F})\right) \supset \mathrm{OL}_{b}\left(\mathfrak{F}_{\varphi}\right)$, и получить требуемую оценку снизу для $\left\|\mathcal{Q}_{\varphi} f\right\|_{\mathrm{OL}\left(\mathfrak{F}_{\varphi}\right)}$, достаточно применить теорему 3.10 .1 к замкнутому множеству $\mathfrak{F}_{\varphi}$ и дробно-линейному преобразованию $\varphi^{-1}$. Теорема доказана.

Приведём ещё один близкий результат.

TeOpema 3.10.3. Пyсmъ $\varphi \in \operatorname{Aut}(\widehat{\mathbb{C}}) \backslash \operatorname{Aut}(\mathbb{C}), a=\varphi(\infty)$. Пyсmъ $\mathfrak{F}-$ saмкнутое подмножество комплексной плоскости $\mathbb{C}$ такое, что а $\notin \mathfrak{F}$. Если $z_{0}$ - одна из ближайших ка точек множества $\mathfrak{F}, \operatorname{mo~}_{\varphi}\left(\mathrm{OL}_{z_{0}}(\mathfrak{F})\right) \subset \mathrm{OL}\left(\mathfrak{F}_{\varphi}\right)$ u

$$
\left\|\mathcal{Q}_{\varphi} f\right\|_{\mathrm{OL}\left(\mathfrak{F}_{\varphi}\right)} \leqslant 5\|f\|_{\mathrm{OL}(\mathfrak{F})} \quad \text { для всех } f \in \mathrm{OL}_{z_{0}}(\mathfrak{F}) .
$$

ДокАзАтельство. Так же, как в доказательстве теоремы 3.10.1, достаточно ограничиться случаем $\varphi(z)=\phi(z) \stackrel{\text { def }}{=} z^{-1}$. Пусть $f \in \mathrm{OL}_{z_{0}}(\mathfrak{F})$ и $\|f\|_{\mathrm{OL}_{z_{0}}(\mathfrak{F})}=1$. Нам нужно доказать, что

$$
\left\|\left(\mathcal{Q}_{\varphi} f\right)(N) R-R\left(\mathcal{Q}_{\varphi} f\right)(N)\right\| \leqslant 5 \max \left\{\|N R-R N\|,\left\|N^{*} R-R N^{*}\right\|\right\}
$$

для любых нормальных операторов $N_{1}$ и $N_{2}$ таких, что $\sigma\left(N_{1}\right), \sigma\left(N_{2}\right) \subset \mathfrak{F}_{\phi}$. Пусть $h$ обозначает то же, что и в доказательстве теоремы 3.10.1. Только теперь мы не можем утверждать, что sup $|h| \leqslant 1$. Имеем

$$
\begin{aligned}
\sup _{z \in \mathfrak{F}_{\phi}}|h(z)| & \leqslant \sup \left\{\left|z f\left(z^{-1}\right)\right|: z \in \phi^{-1}(\mathfrak{F})\right\}=\sup \left\{|z|^{-1}\left|f(z)-f\left(z_{0}\right)\right|: z \in \mathfrak{F}\right\} \\
& \leqslant \sup \left\{|z|^{-1}\left|z-z_{0}\right|: z \in \mathfrak{F}\right\} \leqslant \sup \left\{1+|z|^{-1}\left|z_{0}\right|: z \in \mathfrak{F}\right\}=2 .
\end{aligned}
$$

Повторяя теперь рассуждения доказательства теоремы 3.10.1, получаем

$$
\begin{aligned}
& \left\|\left(\mathcal{Q}_{\varphi} f\right)(N) R-R\left(\mathcal{Q}_{\varphi} f\right)(N)\right\| \\
& \quad \leqslant(1+2 \sup |h(z)|) \max \left\{\|N R-R N\|,\left\|N^{*} R-R N^{*}\right\|\right\} \\
& \quad \leqslant 5 \max \left\{\|N R-R N\|,\left\|N^{*} R-R N^{*}\right\|\right\} .
\end{aligned}
$$

Теорема доказана.

Пример. Пусть $\varphi(z)=z^{-1}, \mathfrak{F}=\mathbb{T}, z_{0}=1, f=1-\bar{z}$. Тогда $f \in \mathrm{OL}_{z_{0}}(\mathbb{T})$ и $\|f\|_{\mathrm{OL}(\mathbb{T})}=1$. Легко проверить, что $\left(\mathcal{Q}_{\varphi} f\right)(z)=z^{3}-z^{2}$ и $\left\|\mathcal{Q}_{\varphi} f\right\|_{\operatorname{Lip}(\mathbb{T})} \geqslant 5$. Тогда

$$
5 \leqslant\left\|\mathcal{Q}_{\varphi} f\right\|_{\mathrm{Lip}(\mathbb{T})} \leqslant\left\|\mathcal{Q}_{\varphi} f\right\|_{\mathrm{OL}(\mathbb{T})} \leqslant\left\|z^{3}\right\|_{\mathrm{OL}(\mathbb{T})}+\left\|z^{2}\right\|_{\mathrm{OL}(\mathbb{T})}=5 .
$$

Этот пример показывает, что константа 5 в теореме 3.10 .3 неулучшаема.

ЗАмЕчАниЕ 1. Можно ввести следующее обобщение оператора $\mathcal{Q}_{\varphi}$, положив

$$
\left(\mathcal{Q}_{n, \varphi} f\right)(z) \stackrel{\text { def }}{=} \begin{cases}\frac{\left|\varphi^{\prime}(z)\right|^{n} f(\varphi(z))}{\left(\varphi^{\prime}(z)\right)^{n+1},} & \text { если } z \in \mathbb{C}, \varphi(z) \in \mathfrak{F} \text { и } \varphi(z) \neq \infty, \\ 0, & \text { если } z \in \mathbb{C} \text { и } \varphi(z)=\infty,\end{cases}
$$

где $n \in \mathbb{Z}$. Имеют место аналоги теорем 3.10.1, 3.10.2 и 3.10 .3 для операторов $\mathcal{Q}_{n, \varphi}$ с константами, зависящими от $n$. Аналоги теорем 3.10 .1 и 3.10 .2 можно найти в [2]. Аналог теоремы 3.10.3 может быть получен таким же образом. 
ЗАмечАниЕ 2. Доказательства теорем 3.10.1, 3.10.2 и 3.10.3 работают и для пространств коммутаторно липшицевых функций. Случай теорем 3.10 .1 и 3.10 .2 разобран в [2]. Разумеется, в случае пространств $\mathrm{CL}(\mathfrak{F})$ речь может идти об обобщениях на операторы $\mathcal{Q}_{n, \varphi}$ (см. замечание 1 ) только для "тонких" множеств $\mathfrak{F}$. Например, если множество $\mathfrak{F}$ содержит внутренние точки, то такие обобщения невозможны, поскольку функции класса CL(F) аналитичны на внутренности множества $\mathfrak{F}$.

Далее нас в основном будут интересовать случаи, когда $\mathfrak{F}=\mathbb{R}$ и $\mathfrak{F}=\mathbb{T}$. В этих случаях имеет место равенство $\mathrm{CL}(\mathfrak{F})=\mathrm{OL}(\mathfrak{F})$ вместе с равенством полунорм.

Отметим, что из теоремы 3.10 .1 вытекает следующее утверждение.

Tеорема 3.10.4. Пусть $\varphi \in \operatorname{Aut}(\widehat{\mathbb{C}})$. Предположим, ито $\varphi(\widehat{\mathbb{R}})=\mathbb{T}$. Тогда

$$
\left\|\mathcal{Q}_{\varphi} f\right\|_{\mathrm{OL}(\mathbb{R})} \leqslant 3\|f\|_{\mathrm{OL}(\mathbb{T})}
$$

для всех $f \in \mathrm{OL}_{a}(\mathbb{T})$, где $a=\varphi(\infty)$.

ДокАЗАтельство. Применим теорему $3.10 .2 \mathrm{k} \mathfrak{F}=\mathbb{T}$. Тогда $\mathfrak{F}_{\varphi}=\mathbb{R} \cup$ $\left\{\varphi^{-1}(\infty)\right\}$, и мы имеем

$$
\left\|\mathcal{Q}_{\varphi} f\right\|_{\mathrm{OL}(\mathbb{R})} \leqslant\left\|\mathcal{Q}_{\varphi} f\right\|_{\mathrm{OL}\left(\mathbb{R} \cup\left\{\varphi^{-1}(\infty)\right\}\right)} \leqslant 3\|f\|_{\mathrm{OL}(\mathbb{T})}
$$

для всех $f \in \mathrm{OL}_{a}(\mathbb{T})$. Теорема доказана.

Положим

$$
(\mathrm{OL})^{\prime}(\mathbb{R}) \stackrel{\text { def }}{=}\left\{f^{\prime}: f \in \mathrm{OL}(\mathbb{R})\right\} \quad \text { и } \quad\left\|f^{\prime}\right\|_{(\mathrm{OL})^{\prime}(\mathbb{R})} \stackrel{\text { def }}{=}\|f\|_{\mathrm{OL}(\mathbb{R})} .
$$

Тогда $(\mathrm{OL})^{\prime}(\mathbb{R})$ - банахово пространство, состоящее из функций, заданных на $\widehat{\mathbb{R}}$.

TeOpema 3.10.5. Пyсmъ $f \in \mathrm{OL}(\mathbb{R})$. Тогдa $(x-a)^{-1}(f(x)-f(a)) \in(\mathrm{OL})^{\prime}(\mathbb{R}) u$

$$
\left\|(x-a)^{-1}(f(x)-f(a))\right\|_{(\mathrm{OL})^{\prime}(\mathbb{R})} \leqslant\|f\|_{\mathrm{OL}(\mathbb{R})} \quad \text { nри всех } a \in \mathbb{R} .
$$

ДоказАтельство. Достаточно рассмотреть случай $a=0$ и $f(0)=0$. Положим

$$
F(x)=\int_{0}^{x} \frac{f(t)}{t} d t=\int_{0}^{1} \frac{f(t x)}{t} d t .
$$

Нам нужно доказать, что $F \in \mathrm{OL}(\mathbb{R})$ и $\|F\|_{\mathrm{OL}(\mathbb{R})} \leqslant\|f\|_{\mathrm{OL}(\mathbb{R})}$. Заметим, что при всех $t$ из $(0,1]$ функция $x \mapsto t^{-1} f(t x)$ принадлежит банахову пространству $\mathrm{OL}_{0}(\mathbb{R})($ см. $(3.10 .1))$ и $\left\|t^{-1} f(t x)\right\|_{\mathrm{OL}(\mathbb{R})}=\|f\|_{\mathrm{OL}(\mathbb{R})}$ при всех $t$ из $(0,1]$. Следовательно,

$$
\|F\|_{\mathrm{OL}(\mathbb{R})} \leqslant \int_{0}^{1}\left\|t^{-1} f(t x)\right\|_{\mathrm{OL}(\mathbb{R})} d t=\|f\|_{\mathrm{OL}(\mathbb{R})} .
$$

Теорема доказана.

ЗАмечАниЕ. Аналогично можно доказать, что для любого замкнутого невырожденного промежутка $J$ и для любой функции $f$ из $\mathrm{OL}(J)$ справедливо неравенство

$$
\left\|(x-a)^{-1}(f(x)-f(a))\right\|_{(\mathrm{OL})^{\prime}(J)} \leqslant\|f\|_{\mathrm{OL}(J)} \quad \text { при всех } a \in J,
$$

где $\mathrm{OL}^{\prime}(J) \stackrel{\text { def }}{=}\left\{g^{\prime}: g \in \mathrm{OL}(J)\right\}$ и $\left\|g^{\prime}\right\|_{\mathrm{OL}^{\prime}(J)} \stackrel{\text { def }}{=}\|g\|_{\mathrm{OL}(J)}$. 
Tеорема 3.10.6. Ecлu $f \in \mathrm{OL}(\mathbb{R}), m o(x-a-b \mathrm{i})^{-1}(f(x)-f(a)) \in(\mathrm{OL})^{\prime}(\mathbb{R}) u$ $\left\|(x-a-b \mathrm{i})^{-1}(f(x)-f(a))\right\|_{(\mathrm{OL})^{\prime}(\mathbb{R})} \leqslant 2\|f\|_{\mathrm{OL}(\mathbb{R})} \quad$ nри всех $a, b \in \mathbb{R}$.

ДокАзАтельство. Достаточно рассмотреть случай, когда $a=0, b=1$, $f(0)=0$ и $\|f\|_{\mathrm{OL}(\mathbb{R})}=1$. Тогда из теоремы 3.10 .5 следует, что

$$
\left\|(x-\mathrm{i})^{-1} f(x)\right\|_{(\mathrm{OL})^{\prime}(\mathbb{R})} \leqslant\left\|(x-\mathrm{i})^{-1} x f(x)\right\|_{\mathrm{OL}(\mathbb{R})} .
$$

Остаётся доказать, что $\left\|(x-\mathrm{i})^{-1} x f(x)\right\|_{\mathrm{OL}(\mathbb{R})} \leqslant 2$. Пусть $A$ и $B$ - самосопряжённые операторы. Имеем

$$
\begin{aligned}
A(A-\mathrm{i} I)^{-1} f(A)-B(B-\mathrm{i} I)^{-1} f(B)= & A(A-\mathrm{i} I)^{-1}(f(A)-f(B)) \\
& +\mathrm{i}(A-\mathrm{i} I)^{-1}(B-A)(B-\mathrm{i} I)^{-1} f(B),
\end{aligned}
$$

откуда следует, что

$$
\begin{aligned}
\left\|A(A-\mathrm{i} I)^{-1} f(A)-B(B-\mathrm{i} I)^{-1} f(B)\right\| & \leqslant\|f(A)-f(B)\|+\|A-B\| \cdot\|g(B)\| \\
& \leqslant 2\|A-B\|,
\end{aligned}
$$

где $g(t)=(t-\mathrm{i})^{-1} f(t)$. Теорема доказана.

СлеДСтвиЕ 3.10.7. Пусть $f \in \mathrm{OL}(\mathbb{R})$. Тогда $(x-a-b \mathrm{i})^{-1} f(x) \in(\mathrm{OL})^{\prime}(\mathbb{R}) u$

$$
\left\|(x-a-b \mathrm{i})^{-1} f(x)\right\|_{(\mathrm{OL})^{\prime}(\mathbb{R})} \leqslant\left(2+\frac{|f(a)|}{|b|}\right)\|f\|_{\mathrm{OL}(\mathbb{R})} \quad \text { npu } a, b \in \mathbb{R}, \quad b \neq 0 .
$$

ДоказАТЕЛЬство. Можно считать, что $a=0, b=1$ и $\|f\|_{\mathrm{OL}(\mathbb{R})}=1$. Используя теорему 3.10 .6 и пример 2 в разделе 1.1 , получаем:

$$
\left\|\frac{f(x)}{x-\mathrm{i}}\right\|_{(\mathrm{OL})^{\prime}(\mathbb{R})} \leqslant\left\|\frac{f(x)-f(0)}{x-\mathrm{i}}\right\|_{(\mathrm{OL})^{\prime}(\mathbb{R})}+|f(0)| \cdot\left\|\frac{1}{x-\mathrm{i}}\right\|_{(\mathrm{OL})^{\prime}(\mathbb{R})} \leqslant 2+|f(0)| .
$$

Следствие доказано.

TEOPEMA 3.10.8. Eсли $h \in \mathrm{OL}^{\prime}(\mathbb{R})$, mо $h \circ \varphi \in \mathrm{OL}^{\prime}(\mathbb{R})$ для всех дробно-линейнъх преобразований $\varphi \in \operatorname{Aut}(\widehat{\mathbb{R}}) u\|h\|_{\mathrm{OL}^{\prime}(\mathbb{R})} / 9 \leqslant\|h \circ \varphi\|_{\mathrm{OL}^{\prime}(\mathbb{R})} \leqslant 9\|h\|_{\mathrm{OL}^{\prime}(\mathbb{R})}$.

ДоказАтельство. Теорема очевидна, если $\varphi \in \operatorname{Aut}(\mathbb{R})$. В этом случае $\|h\|_{\mathrm{OL}^{\prime}(\mathbb{R})}=\|h \circ \varphi\|_{\mathrm{OL}^{\prime}(\mathbb{R})}=\|h\|_{\mathrm{OL}^{\prime}(\mathbb{R})}$. Таким образом, всё сводится к случаю $\varphi(t)=\phi(t) \stackrel{\text { def }}{=} t^{-1}$. Пусть $h=f^{\prime}$ для некоторой функции $f \in \mathrm{OL}(\mathbb{R})$ такой, что $f(0)=0$ и $\|f\|_{\mathrm{OL}(\mathbb{R})}=\|h\|_{\mathrm{OL}^{\prime}(\mathbb{R})}$. Из теоремы 3.10 .2 следует, что $\left\|x^{2} f\left(x^{-1}\right)\right\|_{\mathrm{OL}(\mathbb{R})} \leqslant 3\|h\|_{\mathrm{OL}^{\prime}(\mathbb{R})}$, откуда

$$
\left\|\left(x^{2} f\left(x^{-1}\right)\right)^{\prime}\right\|_{\mathrm{OL}^{\prime}(\mathbb{R})}=\left\|2 x f\left(x^{-1}\right)-h\left(x^{-1}\right)\right\|_{\mathrm{OL}^{\prime}(\mathbb{R})} \leqslant 3\|h\|_{\mathrm{OL}^{\prime}(\mathbb{R})} .
$$

Из теоремы 3.10 .5 вытекает неравенство $\left\|x f\left(x^{-1}\right)\right\|_{\mathrm{OL}^{\prime}(\mathbb{R})} \leqslant\left\|x^{2} f\left(x^{-1}\right)\right\|_{\mathrm{OL}(\mathbb{R})} \leqslant$ $3\|h\|_{\text {OL' }(\mathbb{R})}$. Следовательно,

$$
\left\|h\left(x^{-1}\right)\right\|_{\mathrm{OL}^{\prime}(\mathbb{R})} \leqslant\left\|\left(x^{2} f\left(x^{-1}\right)\right)^{\prime}\right\|_{\mathrm{OL}^{\prime}(\mathbb{R})}+2\left\|x f\left(x^{-1}\right)\right\|_{\mathrm{OL}^{\prime}(\mathbb{R})} \leqslant 9\|h\|_{\mathrm{OL}^{\prime}(\mathbb{R})} .
$$

Применяя это неравенство к $h\left(x^{-1}\right)$, получаем $(1 / 9)\left\|h\left(x^{-1}\right)\right\|_{\mathrm{OL}^{\prime}(\mathbb{R})} \leqslant\|h\|_{\mathrm{OL}^{\prime}(\mathbb{R})}$. Теорема доказана. 


\subsection{1. Пространства $\mathrm{OL}(\mathbb{R})$ и $\mathrm{OL}(\mathbb{T})$}

Основная цель этого и следующего разделов состоит в том, чтобы "пересадить" теорему 3.10 .8 с прямой на окружность.

Легко видеть, что если $f \in \mathrm{OL}(\mathbb{T})$, то $f\left(e^{\mathrm{i} t}\right) \in \mathrm{OL}(\mathbb{R})$ и $\left\|f\left(e^{\mathrm{i} t}\right)\right\|_{\mathrm{OL}(\mathbb{R})} \leqslant$ $\|f\|_{\mathrm{OL}(\mathbb{T})}$. В этом разделе мы покажем, что верно и обратное, т. е. любая $2 \pi$-периодическая функция $F$ из $\mathrm{OL}(\mathbb{R})$ представима в виде $F=f\left(e^{\mathrm{i} t}\right)$, где $f \in \mathrm{OL}(\mathbb{T})$ и $\|f\|_{\mathrm{OL}(\mathbb{T})} \leqslant$ const $\|F\|_{\mathrm{OL}(\mathbb{R})}$. Этот факт нетрудно вывести из леммы 9.8 статьи [11] (см. также лемму 5.7 в [2]).

Лемма 3.11.1. Пусть $h(x, y)=(x-y) /\left(e^{\mathrm{i} x}-e^{\mathrm{i} y}\right) . \quad$ Тогда $\|h\|_{\mathfrak{M}\left(J_{1} \times J_{2}\right)} \leqslant$ $3 \sqrt{2} \pi / 4$ для любых промежутков $J_{1}$ u $J_{2}$ таких, что $J_{1}-J_{2} \subset[-3 \pi / 2,3 \pi / 2]$.

ДокАЗАтельство. Рассмотрим $3 \pi$-периодическую функцию $\xi$ такую, что $\xi(t)=t(2 \sin (t / 2))^{-1}$ при $t \in[-3 \pi / 2,3 \pi / 2]$. Тогда

$$
\|h\|_{\mathfrak{M}\left(J_{1} \times J_{2}\right)}=\left\|e^{\mathrm{i} x / 2} h(x, y) e^{\mathrm{i} y / 2}\right\|_{\mathfrak{M}\left(J_{1} \times J_{2}\right)}=\|\xi(x-y)\|_{\mathfrak{M}\left(J_{1} \times J_{2}\right)},
$$

поскольку $x-y \in[-3 \pi / 2,3 \pi / 2]$ при $x \in J_{1}$ и $y \in J_{2}$. Разложим функцию $\xi$ в ряд Фурье:

$$
\xi(t)=\sum_{n \in \mathbb{Z}} a_{n} e^{2 n \mathrm{i} t / 3}=a_{0}+2 \sum_{n=1}^{\infty} a_{n} \cos \frac{2}{3} n t,
$$

поскольку $a_{n}=a_{-n}$ при всех $n \in \mathbb{Z}$. Ясно, что $a_{0}>0$. Заметим, что функция $\xi$ выпукла на промежутке $[-3 \pi / 2,3 \pi / 2]$. Отсюда следует, что $(-1)^{n} a_{n} \geqslant 0$ при всех натуральных $n$ (см. [29; теорема 35]). Остаётся заметить, что

$$
\begin{aligned}
\|\xi(x-y)\|_{\mathfrak{M}\left(J_{1} \times J_{2}\right)} & \leqslant\|\xi(x-y)\|_{\mathfrak{M}(\mathbb{R} \times \mathbb{R})} \leqslant \sum_{n \in \mathbb{Z}}\left|a_{n}\right| \cdot\left\|e^{2 n \mathrm{i} x / 3} e^{-2 n \mathrm{i} y / 3}\right\|_{\mathfrak{M}(\mathbb{R} \times \mathbb{R})} \\
& =\sum_{n \in \mathbb{Z}}\left|a_{n}\right|=\xi\left(\frac{3 \pi}{2}\right)=\frac{3 \sqrt{2} \pi}{4} .
\end{aligned}
$$

Лемма доказана.

Теорема 3.11.2. Пусть $f$-непрерьвная функиия на $\mathbb{T}$. Тогда

$$
\left\|f\left(e^{\mathrm{i} x}\right)\right\|_{\mathrm{OL}(\mathbb{R})} \leqslant\|f\|_{\mathrm{OL}(\mathbb{T})} \leqslant \frac{3 \sqrt{2} \pi}{2}\left\|f\left(e^{\mathrm{i} x}\right)\right\|_{\mathrm{OL}(\mathbb{R})} .
$$

ДокАзАтельство. Как было отмечено выше, первое неравенство очевидно. Докажем второе неравенство. Положим $g(x) \stackrel{\text { def }}{=} f\left(e^{\mathrm{i} x}\right)$. Можно считать, что $\|g\|_{\mathrm{OL}(\mathbb{R})}<\infty$. Тогда функция $g$ дифференцируема всюду на $\mathbb{R}$. Отсюда вытекает, что функция $f$ имеет производную всюду на $\mathbb{T}$. Из теорем 3.1 .10 и 3.3.6 следует, что

$$
\begin{gathered}
\|g\|_{\mathrm{OL}(\mathbb{R})}=\|\mathfrak{D} g\|_{\mathfrak{M}(\mathbb{R} \times \mathbb{R})}, \\
\|f\|_{\mathrm{OL}(\mathbb{T})}=\|\mathfrak{D} f\|_{\mathfrak{M}(\mathbb{T} \times \mathbb{T})}=\left\|(\mathfrak{D} f)\left(e^{\mathrm{i} x}, e^{\mathrm{i} y}\right)\right\|_{\mathfrak{M}([0,2 \pi) \times[-\pi / 2,3 \pi / 2))} .
\end{gathered}
$$

Таким образом, нам нужно доказать, что

$$
\left\|(\mathfrak{D} f)\left(e^{\mathrm{i} x}, e^{\mathrm{i} y}\right)\right\|_{\mathfrak{M}([0,2 \pi) \times[-\pi / 2,3 \pi / 2))} \leqslant \frac{3 \sqrt{2} \pi}{2}\|\mathfrak{D} g\|_{\mathfrak{M}(\mathbb{R} \times \mathbb{R})} .
$$


Обозначим символом $\chi_{j k}$ характеристическую функцию множества $\boldsymbol{J}_{j, k} \stackrel{\text { def }}{=}$ $[j \pi,(j+1) \pi) \times[k \pi-\pi / 2, k \pi+\pi / 2)$, где $j, k \in \mathbb{Z}$. Заметим, что

$$
\chi_{j k}(x, y)(\mathfrak{D} f)\left(e^{\mathrm{i} x}, e^{\mathrm{i} y}\right)=\chi_{j k}(x, y) h(x, y)(\mathfrak{D} g)(x, y),
$$

где $h$ обозначает то же, что в лемме 3.11.1. Отсюда и из леммы 3.11 .1 следует, что

$$
\left\|(\mathfrak{D} f)\left(e^{\mathrm{i} x}, e^{\mathrm{i} y}\right)\right\|_{\mathfrak{M}\left(\boldsymbol{J}_{j, k}\right)} \leqslant \frac{3 \sqrt{2} \pi}{4}\|\mathfrak{D} g\|_{\mathfrak{M}(\mathbb{R} \times \mathbb{R})}
$$

при $(j, k) \in\{0,1\},(j, k) \neq(1,0)$.

Случай, когда $j=1$ и $k=0$, следует рассмотреть отдельно, поскольку в этом случае $J_{1}-J_{2} \not \subset[-3 \pi / 2,3 \pi / 2]$ и мы не можем применить лемму 3.11 .1 напрямую.

Пусть теперь $j=1$ и $k=0$. Имеем:

$$
\chi_{10}(x+2 \pi, y)(\mathfrak{D} f)\left(e^{\mathrm{i} x}, e^{\mathrm{i} y}\right)=\chi_{10}(x+2 \pi, y) h(x, y)(\mathfrak{D} g)(x, y) .
$$

Применяя лемму 3.11.1, получаем:

$$
\left\|(\mathfrak{D} f)\left(e^{\mathrm{i} x}, e^{\mathrm{i} y}\right)\right\|_{\mathfrak{M}\left(\boldsymbol{J}_{1,0}\right)}=\left\|(\mathfrak{D} f)\left(e^{\mathrm{i} x}, e^{\mathrm{i} y}\right)\right\|_{\mathfrak{M}\left(\boldsymbol{J}_{-1,0}\right)} \leqslant \frac{3 \sqrt{2} \pi}{4}\|\mathfrak{D} g\|_{\mathfrak{M}(\mathbb{R} \times \mathbb{R})} .
$$

Положим также $\boldsymbol{J} \stackrel{\text { def }}{=}[0,2 \pi) \times[-\pi / 2,3 \pi / 2)$. Тогда

$$
\begin{aligned}
\left\|(\mathfrak{D} f)\left(e^{\mathrm{i} x}, e^{\mathrm{i} y}\right)\right\|_{\mathfrak{M}(\boldsymbol{J}) \leqslant} \leqslant & \left\|\left(\chi_{00}(x, y)+\chi_{11}(x, y)\right)(\mathfrak{D} f)\left(e^{\mathrm{i} x}, e^{\mathrm{i} y}\right)\right\|_{\mathfrak{M}(\boldsymbol{J})} \\
& +\left\|\left(\chi_{01}(x, y)+\chi_{10}(x, y)\right)(\mathfrak{D} f)\left(e^{\mathrm{i} x}, e^{\mathrm{i} y}\right)\right\|_{\mathfrak{M}(\boldsymbol{J})} \\
\leqslant & \max \left\{\left\|(\mathfrak{D} f)\left(e^{\mathrm{i} x}, e^{\mathrm{i} y}\right)\right\|_{\mathfrak{M}\left(\boldsymbol{J}_{0,0}\right)},\left\|(\mathfrak{D} f)\left(e^{\mathrm{i} x}, e^{\mathrm{i} y}\right)\right\|_{\mathfrak{M}\left(\boldsymbol{J}_{1,1}\right)}\right\} \\
& \quad+\max \left\{\left\|(\mathfrak{D} f)\left(e^{\mathrm{i} x}, e^{\mathrm{i} y}\right)\right\|_{\mathfrak{M}\left(\boldsymbol{J}_{0,1}\right)},\left\|(\mathfrak{D} f)\left(e^{\mathrm{i} x}, e^{\mathrm{i} y}\right)\right\|_{\mathfrak{M}\left(\boldsymbol{J}_{1,0}\right)}\right\} \\
\leqslant & \frac{3 \sqrt{2} \pi}{2}\|\mathfrak{D} g\|_{\mathfrak{M}(\mathbb{R} \times \mathbb{R}) .}
\end{aligned}
$$

Теорема доказана.

ЗАмЕчАниЕ. Из доказательства теоремы видно, что

$$
\left\|f\left(e^{\mathrm{i} x}\right)\right\|_{\mathrm{OL}(\mathbb{R})} \leqslant\|f\|_{\mathrm{OL}(\mathbb{T})} \leqslant \frac{3 \sqrt{2} \pi}{2}\left\|f\left(e^{\mathrm{i} x}\right)\right\|_{\mathrm{OL}(J)}
$$

для любой функции $f$ из $C(\mathbb{T})$, где $J$ - промежуток длины $3 \pi$.

\subsection{2. Пространства $(\mathrm{OL})^{\prime}(\mathbb{R})$ и $(\mathrm{OL})_{\text {loc }}^{\prime}(\mathbb{T})$}

Пространство $(\mathrm{OL})^{\prime}(\mathbb{R})$ было определено в разделе 3.10 (см. (3.10.3)). Определим теперь пространство $(\mathrm{OL})_{\text {loc }}^{\prime}(\mathbb{T})$ следующим образом:

$$
(\mathrm{OL})_{\text {loc }}^{\prime}(\mathbb{T}) \stackrel{\text { def }}{=}\left\{f: f\left(e^{\mathrm{i} t}\right) \in(\mathrm{OL})^{\prime}(\mathbb{R})\right\} \quad \text { и } \quad\|f\|_{(\mathrm{OL})_{\text {loc }}^{\prime}(\mathbb{T})} \stackrel{\text { def }}{=}\left\|f\left(e^{\mathrm{i} t}\right)\right\|_{(\mathrm{OL})^{\prime}(\mathbb{R})} .
$$

Заметим, что $\|f\|_{L^{\infty}(\mathbb{T})}=\left\|f\left(e^{\mathrm{i} t}\right)\right\|_{L^{\infty}(\mathbb{R})} \leqslant\left\|f\left(e^{\mathrm{i} t}\right)\right\|_{(\mathrm{OL})^{\prime}(\mathbb{R})}=\|f\|_{(\mathrm{OL})_{\text {loc }}^{\prime}(\mathbb{T})}$. 
Нам понадобится следующая элементарная лемма.

Лемма 3.12.1. Пусть $f \in \operatorname{Lip}(\mathbb{T})$. Тогдa $f \in(\mathrm{OL})_{\text {loc }}^{\prime}(\mathbb{T}) u$

$$
\|f\|_{(\mathrm{OL})_{\mathrm{loc}}^{\prime}(\mathbb{T})} \leqslant|\widehat{f}(0)|+\frac{\pi}{\sqrt{3}}\|f\|_{\operatorname{Lip}(\mathbb{T})} .
$$

ДокАЗАТЕЛЬСтво. Заметим, что $\left\|f^{\prime}\right\|_{L^{2}(\mathbb{T})} \leqslant\left\|f^{\prime}\right\|_{L^{\infty}(\mathbb{T})} \leqslant\|f\|_{\operatorname{Lip}(\mathbb{T})}$ и при всех $n \in \mathbb{Z}$ выполнено равенство $\left\|z^{n}\right\|_{(\mathrm{OL})^{\prime}(\mathbb{T})}=1$. Следовательно,

$$
\begin{aligned}
\|f\|_{(\mathrm{OL})_{\mathrm{loc}}^{\prime}(\mathbb{T})} & \leqslant \sum_{n \in \mathbb{Z}}|\widehat{f}(n)| \leqslant|\widehat{f}(0)|+\left(\sum_{n \neq 0} n^{2}|\widehat{f}(n)|^{2}\right)^{1 / 2}\left(\sum_{n \neq 0} \frac{1}{n^{2}}\right)^{1 / 2} \\
& =|\widehat{f}(0)|+\frac{\pi}{\sqrt{3}}\left\|f^{\prime}\right\|_{L^{2}(\mathbb{T})} \leqslant|\widehat{f}(0)|+\frac{\pi}{\sqrt{3}}\left\|f^{\prime}\right\|_{L^{\infty}(\mathbb{T})} \\
& \leqslant|\widehat{f}(0)|+\frac{\pi}{\sqrt{3}}\|f\|_{\operatorname{Lip}(\mathbb{T})} .
\end{aligned}
$$

Лемма доказана.

СЛЕДСТВиЕ 3.12.2. Пространство $\mathrm{OL}(\mathbb{T})$ содержится в $(\mathrm{OL})_{\text {loc }}^{\prime}(\mathbb{T})$ u

$$
\|f\|_{(\mathrm{OL})_{\mathrm{loc}}^{\prime}(\mathbb{T})} \leqslant|\widehat{f}(0)|+\frac{\pi}{\sqrt{3}}\|f\|_{\mathrm{OL}(\mathbb{T})} .
$$

ЗАмЕчАНИЕ. Из доказательства леммы 3.12.1 видно, что

$$
\|f\|_{(\mathrm{OL})_{\mathrm{loc}}^{\prime}(\mathbb{T})} \leqslant|\widehat{f}(0)|+\frac{\pi}{\sqrt{3}}\left\|f^{\prime}\right\|_{L^{2}(\mathbb{T})} .
$$

TеOPEмa 3.12.3. Ecлu $f \in \mathrm{OL}(\mathbb{T}), m o z f^{\prime}(z) \in(\mathrm{OL})_{\mathrm{loc}}^{\prime}(\mathbb{T}) u\left\|z f^{\prime}(z)\right\|_{(\mathrm{OL})_{\mathrm{loc}}^{\prime}(\mathbb{T})} \leqslant$ $\|f\|_{\mathrm{OL}(\mathbb{T})}$. Если $f \in(\mathrm{OL})_{\mathrm{loc}}^{\prime}(\mathbb{T})$ u $\int_{\mathbb{T}} f(z) d \boldsymbol{m}(z)=0$, то существует функиия $F$ из $\mathrm{OL}(\mathbb{T})$ такая, что $z F^{\prime}(z)=f u\|F\|_{\mathrm{OL}(\mathbb{T})} \leqslant$ const $\|f\|_{(\mathrm{OL})_{\mathrm{loc}}^{\prime}(\mathbb{T})}$.

Доказательство. Первое утверждение очевидно, ибо если $f \in \mathrm{OL}(\mathbb{T})$, то $\int_{0}^{x} e^{\mathrm{i} t} f^{\prime}\left(e^{\mathrm{i} t}\right) d t=\mathrm{i} f(1)-\mathrm{i} f\left(e^{\mathrm{i} x}\right), \quad\left\|f^{\prime}\right\|_{(\mathrm{OL})_{\mathrm{loc}}^{\prime}(\mathbb{T})}=\left\|f\left(e^{\mathrm{i} x}\right)\right\|_{\mathrm{OL}(\mathbb{R})} \leqslant\|f\|_{\mathrm{OL}(\mathbb{T})}$.

Докажем второе утверждение. Положим $F\left(e^{\mathrm{i} x}\right) \stackrel{\text { def }}{=} \mathrm{i} \int_{0}^{x} f\left(e^{\mathrm{i} t}\right) d t$. Определение корректно, поскольку $\int_{0}^{2 \pi} f\left(e^{\mathrm{i} t}\right) d t=2 \pi \int_{\mathbb{T}} f(z) d \boldsymbol{m}(z)=0$. Ясно, что $z F^{\prime}(z)=f(z)$. Остаётся заметить, что $\|f\|_{(\mathrm{OL})_{\text {loc }}^{\prime}(\mathbb{T})}=\left\|F\left(e^{\mathrm{i} x}\right)\right\|_{\mathrm{OL}(\mathbb{R})}$, и применить теорему 3.11.2. Теорема доказана.

СледСтвиЕ 3.12.4. Функиия $f$ на $\mathbb{T}$ принадлежит $(\mathrm{OL})_{\mathrm{loc}}^{\prime}(\mathbb{T})$ в том и только том случае, когда она представима в виде $f=\widehat{f}(0)+z F^{\prime}(z)$, где $F \in \mathrm{OL}(\mathbb{T})$. При этом

$$
\|f\|_{(\mathrm{OL})_{\mathrm{loc}}^{\prime}(\mathbb{T})} \leqslant|\widehat{f}(0)|+\|F\|_{\mathrm{OL}(\mathbb{T})} \leqslant \text { const }\|f\|_{(\mathrm{OL})_{\mathrm{loc}}^{\prime}(\mathbb{T})}
$$


ДокАзАтельство. Легко видеть, что $\|1\|_{(\mathrm{OL})_{\mathrm{loc}}^{\prime}(\mathbb{T})}=1$. Отсюда и из теоремы 3.12 .3 следует, что если $f=\widehat{f}(0)+z F^{\prime}(z)$ для некоторой функции $F$ из $\mathrm{OL}(\mathbb{T})$, то $f \in(\mathrm{OL})_{\text {loc }}^{\prime}(\mathbb{T})$ и

$$
\begin{aligned}
\|f\|_{(\mathrm{OL})_{\mathrm{loc}}^{\prime}(\mathbb{T})} & \leqslant\left\|\widehat{f}(0)+z F^{\prime}(z)\right\|_{(\mathrm{OL})_{\mathrm{loc}}^{\prime}(\mathbb{T})} \leqslant|\widehat{f}(0)|+\left\|z F^{\prime}(z)\right\|_{(\mathrm{OL})_{\mathrm{loc}}^{\prime}(\mathbb{T})} \\
& \leqslant\|f\|_{(\mathrm{OL})_{\mathrm{loc}}^{\prime}(\mathbb{T})}+\|F\|_{\mathrm{OL}(\mathbb{T})} \leqslant c\|f\|_{(\mathrm{OL})_{\mathrm{loc}}^{\prime}(\mathbb{T}) .}
\end{aligned}
$$

Пусть $f \in(\mathrm{OL})_{\text {loc }}^{\prime}(\mathbb{T})$. Тогда в силу теоремы 3.12 .3 функция $f-\widehat{f}(0)$ представима в виде $f-\widehat{f}(0)=z F^{\prime}(z)$, где $F \in \mathrm{OL}(\mathbb{T})$. Следствие доказано.

СледСтвиЕ 3.12.5. Если $f \in(\mathrm{OL})_{\text {loc }}^{\prime}(\mathbb{T})$, mo $z^{n} f(z) \in(\mathrm{OL})_{\text {loc }}^{\prime}(\mathbb{T})$ для всех $n$ из $\mathbb{Z}$.

ДокАзАтельство. Достаточно рассмотреть случай, когда $f=z F^{\prime}(z)$, где $F \in \mathrm{OL}(\mathbb{T})$. Тогда $z^{n} f(z)=z^{n+1} F^{\prime}(z)=z\left(z^{n} F(z)\right)^{\prime}-n z^{n} F(z) \in(\mathrm{OL})_{\text {loc }}^{\prime}(\mathbb{T})$, поскольку $z^{n} F(z) \in \mathrm{OL}(\mathbb{T})$ и $\mathrm{OL}(\mathbb{T}) \subset(\mathrm{OL})_{\text {loc }}^{\prime}(\mathbb{T})$ в силу следствия 3.12.2. Следствие доказано.

СледСтвиЕ 3.12.6. Функиия $f$ на $\mathbb{T}$ принадлежит $(\mathrm{OL})_{\mathrm{loc}}^{\prime}(\mathbb{T})$ в том и только том случае, когда она представима в виде $f=\widehat{f}(-1) z^{-1}+F^{\prime}(z)$, где $F \in$ $\mathrm{OL}(\mathbb{T})$, при этом

$$
\|f\|_{(\mathrm{OL})_{\mathrm{loc}}^{\prime}(\mathbb{T})} \leqslant|\widehat{f}(-1)|+\|F\|_{\mathrm{OL}(\mathbb{T})} \leqslant \mathrm{const}\|f\|_{(\mathrm{OL})_{\mathrm{loc}}^{\prime}(\mathbb{T})} .
$$

ДокАЗАТЕЛЬСтво. Положим $g(z) \stackrel{\text { def }}{=} z f(z)$. Тогда $\widehat{f}(-1)=\widehat{g}(0)$ и $g(z)=$ $\widehat{g}(0)+z F^{\prime}(z)$. Остаётся сослаться на следствия 3.12.4 и 3.12.5.

Следующее утверждение очевидно.

ЛЕмма 3.12.7. Пусть $f, g \in \mathrm{OL}(J)$, где $J$ - замкнутый ограниченный промежуток вещественной прямой $\mathbb{R}$. Тогда $f g \in \mathrm{OL}(J)$ и

$$
\|f g\|_{\mathrm{OL}(J)} \leqslant\left(\boldsymbol{m}(J)\|g\|_{\mathrm{OL}(J)}+\max _{J}|g|\right)\|f\|_{\mathrm{OL}(J)} .
$$

Лемма 3.12.8. Пусть $f \in \mathrm{OL}(\mathbb{T}) u \zeta \in \mathbb{T}$. Тогда

$$
\frac{f(z)-f(\zeta)}{z-\zeta} \in(\mathrm{OL})_{\mathrm{loc}}^{\prime}(\mathbb{T}) \quad u \quad\left\|\frac{f(z)-f(\zeta)}{z-\zeta}\right\|_{(\mathrm{OL})_{\mathrm{loc}}^{\prime}(\mathbb{T})} \leqslant \text { const }\|f\|_{\mathrm{OL}(\mathbb{T})}
$$

ДокАЗАтельство. Достаточно рассмотреть случай $\zeta=1$. Можно считать, что $f(1)=0$. Нам нужно оценить $\mathrm{OL}(\mathbb{R})$-полунорму функции $\Phi$, заданной формулой

$$
\Phi(x) \stackrel{\text { def }}{=} \int_{0}^{x} \frac{f\left(e^{\mathrm{i} t}\right)}{e^{\mathrm{i} t}-1} d t .
$$

Ясно, что функция $\Phi$ представима в виде $\Phi(x)=\lambda x+\Phi_{0}(x)$, где $\Phi_{0}-$ функция с периодом $2 \pi$. Имеем

$$
|\lambda|=\left|\frac{1}{2 \pi} \int_{0}^{2 \pi} \frac{f\left(e^{\mathrm{i} t}\right)}{e^{\mathrm{i} t}-1} d t\right| \leqslant \frac{1}{2 \pi} \int_{0}^{2 \pi} \frac{\left|f\left(e^{\mathrm{i} t}\right)-f(1)\right|}{\left|e^{\mathrm{i} t}-1\right|} d t \leqslant\|f\|_{\operatorname{Lip}(\mathbb{T})} \leqslant\|f\|_{\mathrm{OL}(\mathbb{T})} .
$$

Таким образом, остаётся оценить $\mathrm{OL}(\mathbb{R})$-полунорму функции $\Phi_{0}$. 
Оценим сначала $\|\Phi\|_{\mathrm{OL}([-3 \pi / 2,3 \pi / 2])}$. В силу замечания к теореме 3.10 .5 и леммы 3.12.7

$$
\begin{aligned}
\|\Phi\|_{\mathrm{OL}([-3 \pi / 2,3 \pi / 2])} & \leqslant\left\|\frac{t f\left(e^{\mathrm{i} t}\right)}{e^{\mathrm{i} t}-1}\right\|_{\mathrm{OL}([-3 \pi / 2,3 \pi / 2])} \leqslant \operatorname{const}\left\|f\left(e^{\mathrm{i} t}\right)\right\|_{\mathrm{OL}([-3 \pi / 2,3 \pi / 2])} \\
& \leqslant \operatorname{const}\|f\|_{\mathrm{OL}(\mathbb{T})},
\end{aligned}
$$

поскольку функция $t \mapsto t /\left(e^{\mathrm{i} t}-1\right)$ бесконечно дифференцируема на $[-3 \pi / 2,3 \pi / 2]$. Отсюда следует, что $\left\|\Phi_{0}\right\|_{\mathrm{OL}([-3 \pi / 2,3 \pi / 2])} \leqslant$ const $\|f\|_{\mathrm{OL}(\mathbb{T})}$. Используя теперь замечание к теореме 3.11 .2 , получаем

$$
\left\|\Phi_{0}\right\|_{\mathrm{OL}(\mathbb{R})} \leqslant \frac{3 \sqrt{2} \pi}{2}\left\|\Phi_{0}\right\|_{\mathrm{OL}([-3 \pi / 2,3 \pi / 2])} \leqslant \text { const }\|f\|_{\mathrm{OL}(\mathbb{T})} .
$$

Лемма доказана.

Теорема 3.12.9. Пусть $f$ - бункиия, заданная на $\mathbb{T}, a \psi$-дробно-линейное преобразование такое, что $\psi(\widehat{\mathbb{R}})=\mathbb{T}$. Тогда $f \in(\mathrm{OL})_{\text {loc }}^{\prime}(\mathbb{T})$ в том и только том случае, когда $f \circ \psi \in(\mathrm{OL})^{\prime}(\mathbb{R})$, при этом

$$
c_{1}\|f\|_{(\mathrm{OL})_{\mathrm{loc}}^{\prime}(\mathbb{T})} \leqslant\|f \circ \psi\|_{(\mathrm{OL})^{\prime}(\mathbb{R})} \leqslant c_{2}\|f\|_{(\mathrm{OL})_{\mathrm{loc}}^{\prime}(\mathbb{T})},
$$

где $c_{1}$ и $c_{2}$ - абсолютные положительные константы.

ДокАзАтельство. Положим $a=\psi^{-1}(0)$. Легко видеть, что $a \in \mathbb{C} \backslash \mathbb{R}$ и $\psi(z)=\zeta(z-\bar{a})^{-1}(z-a)$ при всех $z \in \widehat{\mathbb{C}}$, где $|\zeta|=1$. Не умаляя общности, можно считать, что $\zeta=1$. Докажем сначала второе неравенство. Пусть $f \in$ $(\mathrm{OL})_{\text {loc }}^{\prime}(\mathbb{T})$. В силу следствия 3.12 .6 функция $f$ представима в виде $f(z)=$ $\widehat{f}(-1) z^{-1}+F^{\prime}(z)$, где $F \in \mathrm{OL}(\mathbb{T})$, при этом $|\widehat{f}(-1)|+\|F\|_{\mathrm{OL}(\mathbb{T})} \leqslant c\|f\|_{(\mathrm{OL})_{\text {loc }}^{\prime}(\mathbb{T})}$. Имеем

$$
\begin{aligned}
\|f \circ \psi\|_{(\mathrm{OL})^{\prime}(\mathbb{R})} & =\left\|\widehat{f}(-1) \frac{1}{\psi}+F^{\prime} \circ \psi\right\|_{(\mathrm{OL})^{\prime}(\mathbb{R})} \\
& \leqslant c\left\|\frac{1}{\psi}\right\|_{(\mathrm{OL})^{\prime}(\mathbb{R})}\|f\|_{(\mathrm{OL})_{\mathrm{loc}}^{\prime}(\mathbb{T})}+\left\|F^{\prime} \circ \psi\right\|_{(\mathrm{OL})^{\prime}(\mathbb{R})} .
\end{aligned}
$$

Заметим, что

$$
\left\|\frac{1}{\psi}\right\|_{(\mathrm{OL})^{\prime}(\mathbb{R})}=\left\|(t-a)^{-1}(t-\bar{a})\right\|_{(\mathrm{OL})^{\prime}(\mathbb{R})} \leqslant 1+2|\operatorname{Im} a| \cdot\left\|(t-a)^{-1}\right\|_{(\mathrm{OL})^{\prime}(\mathbb{R})} \leqslant 3
$$

что легко следует из примера 2 в разделе 1.1 . Оценим теперь $\left\|F^{\prime} \circ \psi\right\|_{(\mathrm{OL})^{\prime}(\mathbb{R})}$. Функцию $F$ выберем так, чтобы $F(1)=F(\psi(\infty))=0$. Из теоремы 3.10 .4 следует, что

$$
\left\|\frac{F \circ \psi}{\psi^{\prime}}\right\|_{\mathrm{OL}(\mathbb{R})}=\left\|\mathcal{Q}_{\psi} F\right\|_{\mathrm{OL}(\mathbb{R})} \leqslant 3\|F\|_{\mathrm{OL}(\mathbb{T})} \leqslant \operatorname{const}\|f\|_{(\mathrm{OL})_{\mathrm{loc}}^{\prime}(\mathbb{T})} .
$$

Следовательно,

$$
\left\|F^{\prime} \circ \psi-\frac{(F \circ \psi) \psi^{\prime \prime}}{\left(\psi^{\prime}\right)^{2}}\right\|_{(\mathrm{OL})^{\prime}(\mathbb{R})}=\left\|\left(\frac{F \circ \psi}{\psi^{\prime}}\right)^{\prime}\right\|_{(\mathrm{OL})^{\prime}(\mathbb{R})} \leqslant \text { const }\|f\|_{(\mathrm{OL})_{\mathrm{loc}}^{\prime}(\mathbb{T})} .
$$


Остаётся оценить

$$
\left\|\frac{(F \circ \psi) \psi^{\prime \prime}}{\left(\psi^{\prime}\right)^{2}}\right\|_{(\mathrm{OL})^{\prime}(\mathbb{R})}=\left\|\frac{\left(\mathcal{Q}_{\psi} F\right) \psi^{\prime \prime}}{\psi^{\prime}}\right\|_{(\mathrm{OL})^{\prime}(\mathbb{R})}=2\left\|(z-\bar{a})^{-1} \mathcal{Q}_{\psi} F\right\|_{(\mathrm{OL})^{\prime}(\mathbb{R})^{\circ}}
$$

Используя теорему 3.10.6, получаем:

$$
\begin{aligned}
\left\|(z-\bar{a})^{-1} \mathcal{Q}_{\psi} F\right\|_{(\mathrm{OL})^{\prime}(\mathbb{R}) \leqslant} \leqslant & \left\|(z-\bar{a})^{-1}\left(\mathcal{Q}_{\psi} F-\left(\mathcal{Q}_{\psi} F\right)(\operatorname{Re} a)\right)\right\|_{(\mathrm{OL})^{\prime}(\mathbb{R})} \\
& +\left|\left(\mathcal{Q}_{\psi} F\right)(\operatorname{Re} a)\right| \cdot\left\|(z-\bar{a})^{-1}\right\|_{(\mathrm{OL})^{\prime}(\mathbb{R})} \\
\leqslant & 2\left\|\mathcal{Q}_{\psi} F\right\|_{\mathrm{OL}(\mathbb{R})}+2|F(-1)| \cdot|\operatorname{Im} a| \cdot\left\|(z-\bar{a})^{-1}\right\|_{(\mathrm{OL})^{\prime}(\mathbb{R})} \\
\leqslant & 6\|F\|_{\mathrm{OL}(\mathbb{T})}+2|F(-1)-F(1)| \leqslant 10\|F\|_{\mathrm{OL}(\mathbb{T})} \\
\leqslant & \operatorname{const}\|f\|_{(\mathrm{OL})_{\mathrm{loc}}^{\prime}(\mathbb{T})} .
\end{aligned}
$$

Докажем теперь первое неравенство. Возьмём функцию $g \in \mathrm{OL}(\mathbb{R})$ такую, что $g^{\prime}(t) \stackrel{\text { def }}{=} f(\psi(t)) \in(\mathrm{OL})^{\prime}(\mathbb{R})$ и $g(\operatorname{Re} a)=0$. Пусть $\varkappa-$ дробно-линейное преобразование, обратное преобразованию $\psi$, т. е. $\varkappa(z)=(1-z)^{-1}(a-\bar{a} z)$. Из теоремы 3.10.3 вытекает, что

$$
\left\|(2 \operatorname{Im} a)^{-1}(1-z)^{2} g(\varkappa(z))\right\|_{\mathrm{OL}(\mathbb{T})} \leqslant 5\|g\|_{\mathrm{OL}(\mathbb{R})} .
$$

Поэтому $\left\|(\operatorname{Im} a)^{-1}(z-1) g(\varkappa(z))+f(z)\right\|_{(\mathrm{OL})_{\mathrm{loc}}^{\prime}(\mathbb{T})} \leqslant 5\|g\|_{\mathrm{OL}(\mathbb{R})}=5\|f \circ \psi\|_{(\mathrm{OL})^{\prime}(\mathbb{R})}$ в силу следствия 3.12.6. Остаётся доказать, что

$$
\left\|(\operatorname{Im} a)^{-1}(z-1) g(\varkappa(z))\right\|_{(\mathrm{OL})_{\mathrm{loc}}^{\prime}(\mathbb{T})} \leqslant \text { const }\|f \circ \psi\|_{(\mathrm{OL})^{\prime}(\mathbb{R})} .
$$

Это мгновенно вытекает из (3.12.2) и леммы 3.12.8. Теорема доказана.

Tеорема 3.12.10. Пусть $f \in(\mathrm{OL})_{\mathrm{loc}}^{\prime}(\mathbb{T})$, a $\varphi$ - дробно-линейное преобразование такое, что $\varphi(\mathbb{T})=\mathbb{T}$. Тогда $f \circ \varphi \in(\mathrm{OL})_{\mathrm{loc}}^{\prime}(\mathbb{T})$ u $c^{-1}\|f\|_{(\mathrm{OL})_{\mathrm{loc}}^{\prime}(\mathbb{T})} \leqslant$ $\|f \circ \varphi\|_{(\mathrm{OL})_{\mathrm{loc}}^{\prime}(\mathbb{T})} \leqslant c\|f\|_{(\mathrm{OL})_{\mathrm{loc}}^{\prime}(\mathbb{T})}$ для некоторого положстелъного числа $c$.

ДокАЗАТЕльство. Эта теорема по существу является аналогом для окружности $\mathbb{T}$ теоремы 3.10.8, в которой речь шла о прямой $\mathbb{R}$. Теорема 3.12 .9 позволяет “пересадить" теорему 3.10 .8 с прямой $\mathbb{R}$ на окружность $\mathbb{T}$.

\subsection{3. Вокруг достаточного условия Арази-Бартона-Фридмана}

В этом разделе мы рассмотрим достаточное условие для операторной липшицевости на окружности $\mathbb{T}$, найденное в работе Дж. Арази, Т. Бартона и Я. Фридмана [15], а также его аналог для прямой $\mathbb{R}$. Следуя [3], мы покажем, как вывести эти достаточные условия из теоремы 3.8.1. Далее мы введём понятия карлесоновой меры в сильном смысле и переформулируем эти достаточные условия в терминах таких мер. Мы также покажем, как можно вывести из них достаточные условия в терминах классов Бесова (см. раздел 1.6). Начнём со случая прямой.

Положим $(\mathrm{CL})^{\prime}\left(\mathbb{C}_{+}\right) \stackrel{\text { def }}{=}\left\{g^{\prime}: g \in \mathrm{CL}\left(\mathbb{C}_{+}\right)\right\}$и $\left\|g^{\prime}\right\|_{(\mathrm{CL})^{\prime}\left(\mathbb{C}_{+}\right)}=\|g\|_{\mathrm{CL}\left(\mathbb{C}_{+}\right)}$. Ясно, что $(\mathrm{CL})^{\prime}\left(\mathbb{C}_{+}\right)$- банахово пространство. Заметим, что функции класca $(\mathrm{CL})^{\prime}\left(\mathbb{C}_{+}\right)$заданы всюду на $\operatorname{clos} \mathbb{C}_{+} \cup\{\infty\}$. Легко видеть, что для любой функции $g$ из $\mathrm{CL}\left(\mathbb{C}_{+}\right)$интеграл Пуассона функции $g^{\prime} \mid \mathbb{R}$ совпадает с $g^{\prime} \mid \mathbb{C}_{+}$. 
Для этого достаточно заметить, что при всех $t>0$ интеграл Пуассона функции $t^{-1}\left(g_{t}-g\right) \mid \mathbb{R}$ совпадает с функцией $t^{-1}\left(g_{t}-g\right) \mid \mathbb{C}_{+}$, где $g_{t}(z) \stackrel{\text { def }}{=} g(z+t)$, и перейти к пределу при $t \rightarrow 0^{+}$. Обозначим через $(\mathrm{OL})_{+}(\mathbb{R})$ пространство всех функций $f \in \mathrm{OL}(\mathbb{R})$, допускающих аналитическое продолжение в верхнюю полуплоскость $\mathbb{C}_{+}$, непрерывное вплоть до границы. Положим $(\mathrm{OL})_{+}^{\prime}(\mathbb{R}) \stackrel{\text { def }}{=}\left\{g^{\prime}: g \in\right.$ $\left.\mathrm{OL}_{+}(\mathbb{R})\right\}$.

Из теоремы 3.9 .7 следует, что пространство $(\mathrm{OL})_{+}^{\prime}(\mathbb{R})$ отождествляется естественным образом с пространством $(\mathrm{CL})^{\prime}\left(\mathbb{C}_{+}\right)$. При этом

$$
\left\|f^{\prime}\right\|_{(\mathrm{OL})^{\prime}(\mathbb{R})}=\|f\|_{\mathrm{OL}(\mathbb{R})}=\|f\|_{\mathrm{CL}\left(\mathbb{C}_{+}\right)}=\left\|f^{\prime}\right\|_{(\mathrm{CL})^{\prime}\left(\mathbb{C}_{+}\right)} \quad \text { для всех } f \in(\mathrm{CL})\left(\mathbb{C}_{+}\right) .
$$

Аналог теоремы Арази-Бартона-Фридмана для полуплоскости можно сформулировать следующим образом.

Теорема 3.13.1. Пусть $f$ - бункиия, аналитическая в верхней полуплоскости. Предположим, что $\sup _{t \in \mathbb{R}} \int_{\mathbb{C}_{+}} \frac{(\operatorname{Im} w)\left|f^{\prime}(w)\right| d \boldsymbol{m}_{2}(w)}{|t-w|^{2}}<+\infty$. Тогда функция $f$ имеет конечные угловые граничные значения вскоду на $\widehat{\mathbb{R}}$, которые мы будем обозначать той же буквой $f, f \in(\mathrm{CL})^{\prime}\left(\mathbb{C}_{+}\right)$u

$$
\|f-f(\infty)\|_{(\mathrm{CL})^{\prime}\left(\mathbb{C}_{+}\right)} \leqslant \frac{2}{\pi} \sup _{t \in \mathbb{R}} \int_{\mathbb{C}_{+}} \frac{(\operatorname{Im} w)\left|f^{\prime}(w)\right| d \boldsymbol{m}_{2}(w)}{|t-w|^{2}}
$$

ЛЕмма 3.13.2. Пусть $f$ - аналитическая в верхней полуплоскости $\mathbb{C}_{+}$ функция. Предположим, что $\int_{\mathbb{C}_{+}}(\operatorname{Im} w)\left(1+|w|^{2}\right)^{-1}\left|f^{\prime}(w)\right| d \boldsymbol{m}_{2}(w)<+\infty$. Тогда функиия $f$ имеет конечное угловое значение $f(\infty)$ в бесконечности и

$$
f(z)-f(\infty)=\frac{2 \mathrm{i}}{\pi} \int_{\mathbb{C}_{+}} \frac{(\operatorname{Im} w) f^{\prime}(w) d \boldsymbol{m}_{2}(w)}{(\bar{w}-z)^{2}} \quad \text { при всех } z \in \mathbb{C}_{+} .
$$

ДоКАЗАТЕЛЬСТво. Положим

$$
g(z) \stackrel{\text { def }}{=} \frac{2 \mathrm{i}}{\pi} \int_{\mathbb{C}_{+}} \frac{(\operatorname{Im} w) f^{\prime}(w) d \boldsymbol{m}_{2}(w)}{(\bar{w}-z)^{2}} \quad \text { для } z \in \mathbb{C}_{+} .
$$

Ясно, что функция $g$ аналитична в $\mathbb{C}_{+}$и $g^{\prime}(z)=\frac{4 \mathrm{i}}{\pi} \int_{\mathbb{C}_{+}} \frac{f^{\prime}(w) d \boldsymbol{m}_{2}(w)}{(\bar{w}-z)^{3}}=f^{\prime}(z)$ для всех $z \in \mathbb{C}_{+}$, где последнее равенство следует из того, что $4 \mathrm{i}(\pi)^{-1}(\bar{w}-z)^{-3}$ воспроизводящее ядро для пространства Бергмана, состоящего из аналитических в $\mathbb{C}_{+}$функций класса $L^{2}\left(\mathbb{C}_{+}, y d \boldsymbol{m}_{2}(x+\mathrm{i} y)\right)$. Это хорошо известный и легко проверяемый факт. Остаётся доказать, что некасательный предел функции $g$ в бесконечности равен нулю. Из равенства

$$
g(z)=\frac{2 \mathrm{i}}{\pi} \int_{\mathbb{C}_{+}}\left(\frac{\bar{w}-\mathrm{i}}{\bar{w}-z}\right)^{2} \frac{f^{\prime}(w) d \boldsymbol{m}_{2}(w)}{(\bar{w}-\mathrm{i})^{2}}
$$

и теоремы Лебега о предельном переходе под знаком интеграла следует, что сужение функции $g(z)$ на любую полуплоскость $\varepsilon \mathrm{i}+\mathbb{C}_{+}$, где $\varepsilon>0$, стремится к нулю при $|z| \rightarrow \infty$. Лемма доказана.

\footnotetext{
${ }^{4}$ Под $f(\infty)$ понимается $\lim f(z)$, когда $|z| \rightarrow \infty$ и $z$ остаётся в любом замкнутом угле с вершиной в $\mathbb{R}$, все точки которого, кроме вершины, лежат в $\mathbb{C}_{+}$.
} 
ДОКАЗАТЕЛЬСТВО ТЕОРЕМЫ 3.13.1. Положим

$F(z) \stackrel{\text { def }}{=} \frac{2 \mathrm{i}}{\pi} \int_{\mathbb{C}_{+}}(\operatorname{Im} w) f^{\prime}(w)\left(\frac{1}{\bar{w}-z}-\frac{1}{\bar{w}}\right) d \boldsymbol{m}_{2}(w)=\frac{2 \mathrm{i} z}{\pi} \int_{\mathbb{C}_{+}} \frac{(\operatorname{Im} w) f^{\prime}(w) d \boldsymbol{m}_{2}(w)}{\bar{w}(\bar{w}-z)}$

для всех $z \in \mathbb{C}$ таких, что $\operatorname{Im} z \geqslant 0$. Сходимость интегралов при вещественных $z$ вытекает из неравенства Коши-Буняковского, если принять во внимание оче$\begin{aligned} & \text { видное неравенство } \\ & \text { метим, что }\end{aligned} \int_{\mathbb{C}_{+}} \frac{(\operatorname{Im} w)\left|f^{\prime}(w)\right| d \boldsymbol{m}_{2}(w)}{|z-\bar{w}|^{2}} \leqslant \int_{\mathbb{C}_{+}} \frac{(\operatorname{Im} w)\left|f^{\prime}(w)\right| d \boldsymbol{m}_{2}(w)}{|\operatorname{Re} z-w|^{2}}$. За-

$$
F^{\prime}(z)=\frac{2 \mathrm{i}}{\pi} \int_{\mathbb{C}_{+}} \frac{(\operatorname{Im} w) f^{\prime}(w) d \boldsymbol{m}_{2}(w)}{(\bar{w}-z)^{2}}=f(z)-f(\infty)
$$

в силу леммы 3.13.2. Рассмотрим меру Радона $\mu$ в нижней полуплоскости $\mathbb{C}_{-}$:

$$
d \mu(w) \stackrel{\text { def }}{=} \frac{2 \mathrm{i}}{\pi}(\operatorname{Im} \bar{w}) f^{\prime}(\bar{w}) d \boldsymbol{m}_{2}(w) .
$$

Тогда $F(z)=\widehat{\mu}_{0}(z)$ при всех $z$ таких, что $\operatorname{Im} z \geqslant 0$, и

$$
\begin{aligned}
\|\mu\|_{\mathscr{M}\left(\mathbb{C}_{-}\right)} & =\frac{2}{\pi} \sup _{z \in \mathbb{C}_{+}} \int_{\mathbb{C}_{+}} \frac{(\operatorname{Im} w)\left|f^{\prime}(w)\right| d \boldsymbol{m}_{2}(w)}{|\bar{z}-w|^{2}} \\
& =\frac{2}{\pi} \sup _{t \in \mathbb{R}} \int_{\mathbb{C}_{+}} \frac{(\operatorname{Im} w)\left|f^{\prime}(w)\right| d \boldsymbol{m}_{2}(w)}{|t-w|^{2}} .
\end{aligned}
$$

Теперь из теоремы 3.8.1 следует, что

$$
\|f-f(\infty)\|_{(\mathrm{CL})^{\prime}\left(\mathbb{C}_{+}\right)}=\|F\|_{\mathrm{CL}\left(\mathbb{C}_{+}\right)} \leqslant \frac{2}{\pi} \sup _{t \in \mathbb{R}} \int_{\mathbb{C}_{+}} \frac{(\operatorname{Im} w)\left|f^{\prime}(w)\right| d \boldsymbol{m}_{2}(w)}{|t-w|^{2}} .
$$

Теорема доказана.

Обозначим символом $\mathcal{P} M\left(\mathbb{C}_{+}\right)$пространство всех комплексных гармонических функций $u$, заданных в верхней полуплоскости $\mathbb{C}_{+}$и таких, что

$$
\|u\|_{\mathcal{P} M\left(\mathbb{C}_{+}\right)} \stackrel{\text { def }}{=} \sup _{y>0} \int_{\mathbb{R}}|u(x+\mathrm{i} y)| d x<+\infty .
$$

Хорошо известно (см., например, [71; теоремы 2.3 и 2.5 гл. II]), что пространство $\mathcal{P} M\left(\mathbb{C}_{+}\right)$совпадает с множеством всех функций $u$, представимых в виде

$$
u(z)=(\mathcal{P} \nu)(z) \stackrel{\text { def }}{=} \frac{1}{\pi} \int_{\mathbb{R}} \frac{\operatorname{Im} z d \nu(t)}{|z-t|^{2}}, \quad z \in \mathbb{C}_{+},
$$

где $\nu$ - комплексная борелевская мера на $\mathbb{R}$, при этом $\|u\|_{\mathcal{P} M\left(\mathbb{C}_{+}\right)}=\|\nu\|_{M(\mathbb{R})} \stackrel{\text { def }}{=}$ $|\mu|(\mathbb{R})$.

Обозначим символом $\mathcal{P} L\left(\mathbb{C}_{+}\right)$подпространство пространства $\mathcal{P} M\left(\mathbb{C}_{+}\right)$, состоящее из всех функций $u \in \mathcal{P} L\left(\mathbb{C}_{+}\right)$, которые соответствуют абсолютно непрерывным мерам $\nu$. 
Положительную меру $\mu$ на $\mathbb{C}_{+}$будем называть мерой Карлесона в сильном смысле, если $\int_{\mathbb{C}_{+}}|u(z)| d \mu(z)<+\infty$ для любой функции $u \in \mathcal{P} M\left(\mathbb{C}_{+}\right)$. Заметим, что пространство $\mathcal{P} M\left(\mathbb{C}_{+}\right)$содержит класс Харди $H^{1}$ в верхней полуплоскости $\mathbb{C}_{+}$. Отсюда следует, что любая мера Карлесона в сильном смысле является мерой Карлесона в обычном смысле. Обозначим символом $\mathrm{CM}_{\mathrm{S}}\left(\mathbb{C}_{+}\right)$ пространство всех мер Радона $\mu$ в $\mathbb{C}_{+}$таких, что $|\mu|-$ мера Карлесона в сильном смысле. Положим

$$
\|\mu\|_{\mathrm{CM}_{\mathrm{s}}\left(\mathbb{C}_{+}\right)} \stackrel{\text { def }}{=} \sup \left\{\int_{\mathbb{C}_{+}}|u(z)| d \mu(z): u \in \mathcal{P} M\left(\mathbb{C}_{+}\right),\|u\|_{\mathcal{P} M\left(\mathbb{C}_{+}\right)} \leqslant 1\right\} .
$$

Легко видеть, что

$$
\|\mu\|_{\mathrm{CM}_{\mathrm{s}}\left(\mathbb{C}_{+}\right)}=\sup \left\{\int_{\mathbb{C}_{+}}|u(z)| d \mu(z): u \in \mathcal{P} L\left(\mathbb{C}_{+}\right),\|u\|_{\mathcal{P} M\left(\mathbb{C}_{+}\right)} \leqslant 1\right\}
$$

И

$$
\|\mu\|_{\mathrm{CM}_{\mathrm{s}}\left(\mathbb{C}_{+}\right)}=\frac{1}{\pi} \sup _{t \in \mathbb{R}} \int_{\mathbb{C}_{+}} \frac{(\operatorname{Im} w) d \mu(w)}{|t-w|^{2}}=\frac{1}{\pi} \sup _{z \in \mathbb{C}_{+}} \int_{\mathbb{C}_{+}} \frac{(\operatorname{Im} w) d \mu(w)}{|\bar{z}-w|^{2}} .
$$

Теперь аналог достаточного условия Арази-Бартона-Фридмана для полуплоскости можно переформулировать следующим образом.

ТЕОрема 3.13.3. Пусть $f$ - функиия, аналитическая в верхней полуплоскости $\mathbb{C}_{+}$. Предположим, что $\left|f^{\prime}\right| d \boldsymbol{m}_{2} \in \mathrm{CM}_{\mathrm{s}}\left(\mathbb{C}_{+}\right)$. Тогда функиия $f$ имеет конечные граничные значения всюду на расширенной числовой прямой (которые мы обозначаем той же буквой $f), f \in(\mathrm{CL})^{\prime}\left(\mathbb{C}_{+}\right)$и

$$
\|f-f(\infty)\|_{(\mathrm{CL})^{\prime}\left(\mathbb{C}_{+}\right)} \leqslant 2\left\|f^{\prime} d \boldsymbol{m}_{2}\right\|_{\mathrm{CM}_{\mathrm{s}}\left(\mathbb{C}_{+}\right)} .
$$

Аналогично можно получить ещё один вариант теоремы Арази-БартонаФридмана. В следующей теореме, как и во всём этом разделе, $\|(\nabla u)(a)\|$ будет обозначать операторную норму дифференциала $d_{a} u$ функции $u$ в точке $a$.

Теорема 3.13.4. Пусть и - (комплексная) гармоническая функиия в верхней полуплоскости $\mathbb{C}_{+}$. Предположим, ито $\|\nabla u\| d \boldsymbol{m}_{2} \in \mathrm{CM}_{\mathrm{S}}\left(\mathbb{C}_{+}\right)$. Тогда функиия и имеет некасательные граничные значения всюду на $\widehat{\mathbb{R}}, u \mid \mathbb{R} \in(\mathrm{OL})^{\prime}(\mathbb{R})$ u

$$
\|u-u(\infty)\|_{(\mathrm{OL})^{\prime}(\mathbb{R})} \leqslant 2\|\| \nabla u\left\|d \boldsymbol{m}_{2}\right\|_{\mathrm{CM}_{\mathrm{s}}\left(\mathbb{C}_{+}\right)} .
$$

ДоказАтельство. Рассмотрим аналитические в $\mathbb{C}_{+}$функции $f$ и $g$ такие, что $f+\bar{g}=u$. Тогда $f^{\prime}=\frac{\partial u}{\partial z}=\frac{1}{2}\left(\frac{\partial u}{\partial x}-\mathrm{i} \frac{\partial u}{\partial y}\right)$ и $\overline{g^{\prime}}=\frac{\partial u}{\partial \bar{z}}=\frac{1}{2}\left(\frac{\partial u}{\partial x}+\mathrm{i} \frac{\partial u}{\partial y}\right)$. Положим

$$
\begin{aligned}
F(z) & \stackrel{\text { def }}{=} \frac{2 \mathrm{i}}{\pi} \int_{\mathbb{C}_{+}}(\operatorname{Im} w) f^{\prime}(w)\left(\frac{1}{\bar{w}-z}-\frac{1}{\bar{w}}\right) d \boldsymbol{m}_{2}(w) \\
& =\frac{2 \mathrm{i} z}{\pi} \int_{\mathbb{C}_{+}} \frac{(\operatorname{Im} w) f^{\prime}(w) d \boldsymbol{m}_{2}(w)}{\bar{w}(\bar{w}-z)} \\
G(z) & \stackrel{\text { def }}{=} \frac{2 \mathrm{i}}{\pi} \int_{\mathbb{C}_{+}}(\operatorname{Im} w) g^{\prime}(w)\left(\frac{1}{\bar{w}-z}-\frac{1}{\bar{w}}\right) d \boldsymbol{m}_{2}(w) \\
& =\frac{2 \mathrm{i} z}{\pi} \int_{\mathbb{C}_{+}} \frac{(\operatorname{Im} w) g^{\prime}(w) d \boldsymbol{m}_{2}(w)}{\bar{w}(\bar{w}-z)}
\end{aligned}
$$


для всех $z \in \mathbb{C}$ таких, что $\operatorname{Im} z \geqslant 0$. Применяя тождество (3.13.1) и аналогичное тождество для функции $G$, получаем

$$
\begin{aligned}
u(x)-u(\infty) & =F^{\prime}(x)+\bar{G}^{\prime}(x) \\
& =\frac{2 \mathrm{i}}{\pi} \int_{\mathbb{C}_{+}} \frac{(\operatorname{Im} w) f^{\prime}(w) d \boldsymbol{m}_{2}(w)}{(\bar{w}-x)^{2}}+\frac{2 \mathrm{i}}{\pi} \int_{\mathbb{C}_{+}} \frac{(\operatorname{Im} w) \overline{g^{\prime}(w)} d \boldsymbol{m}_{2}(w)}{(w-x)^{2}} .
\end{aligned}
$$

Применяя теорему 3.8.1, получаем:

$$
\begin{aligned}
\| u- & u(\infty) \|_{(\mathrm{OL})^{\prime}(\mathbb{R})} \\
& \leqslant \frac{2}{\pi} \sup _{x \in \mathbb{R}}\left(\int_{\mathbb{C}_{+}} \frac{(\operatorname{Im} w)\left|f^{\prime}(w)\right| d \boldsymbol{m}_{2}(w)}{|x-\bar{w}|^{2}}+\int_{\mathbb{C}_{+}} \frac{(\operatorname{Im} w)\left|g^{\prime}(w)\right| d \boldsymbol{m}_{2}(w)}{|x-w|^{2}}\right) \\
& =\sup _{x \in \mathbb{R}} \int_{\mathbb{C}_{+}} \frac{(\operatorname{Im} w)\left(\left|f^{\prime}(w)\right|+\left|g^{\prime}(w)\right|\right) d \boldsymbol{m}_{2}(w)}{|x-w|^{2}} .
\end{aligned}
$$

Остаётся заметить, что $\left|f^{\prime}(w)\right|+\left|g^{\prime}(w)\right|=\|(\nabla u)(w)\|$ для всех $w$ из $\mathbb{C}_{+}$, поскольку операторная норма линейного отображения $h \mapsto \alpha h+\beta \bar{h}$ равна $|\alpha|+|\beta|$. Теорема доказана.

СлеДСтвиЕ 3.13.5. Пусть $f \in \operatorname{Lip}(\mathbb{R}) u\|\operatorname{Hess} \mathcal{P} f\| d \boldsymbol{m}_{2} \in \mathrm{CM}_{\mathrm{s}}\left(\mathbb{C}_{+}\right)$. Тогда $f \in \mathrm{OL}(\mathbb{R})$.

Теперь мы покажем, что из достаточного условия Арази-Бартона-Фридмана вытекает достаточное условие для операторной липшицевости, полученное в [56] и [58] (см. теорему 1.6.1 в настоящем обзоре).

Чтобы получить это достаточное условие, нам понадобится следующее элементарное неравенство:

$$
\left\|\varphi d \boldsymbol{m}_{2}\right\|_{\mathrm{CM}_{\mathrm{s}}\left(\mathbb{C}_{+}\right)} \leqslant \int_{0}^{\infty} \operatorname{ess} \sup \{\varphi(x+\mathrm{i} y): x \in \mathbb{R}\} d y
$$

для любой измеримой неотрицательной функции $\varphi$ на $\mathbb{C}_{+}$.

Переходим теперь к альтернативному доказательству достаточного условия, полученного в [56], [58].

TEOPEMA 3.13.6. Пусть $f \in B_{\infty, 1}^{1}(\mathbb{R})$. Тогда $f \in \mathrm{OL}(\mathbb{R})$.

ДокаЗАтельство. Ясно, что $f^{\prime} \in L^{\infty}(\mathbb{R})$. Пусть $u$ - интеграл Пуассона функции $f^{\prime}$. Как известно (см. раздел 2), включение $f \in B_{\infty, 1}^{1}(\mathbb{R})$ равносильно следующему условию: $\int_{0}^{\infty} \sup _{x \in \mathbb{R}}\|\nabla u(x+\mathrm{i} y)\| d y<+\infty$. Остаётся воспользоваться неравенством (3.13.2) и сослаться на теорему 3.13.4.

Рассмотрим теперь случай круга. Положим $(\mathrm{CL})^{\prime}(\mathbb{D}) \stackrel{\text { def }}{=}\left\{g^{\prime}: g \in \mathrm{CL}\left(\mathbb{C}_{+}\right)\right\}$и $\left\|g^{\prime}\right\|_{(\mathrm{CL})^{\prime}(\mathbb{D})}=\|g\|_{\mathrm{CL}(\mathbb{D})}$.

Обозначим через $\mathcal{P} M(\mathbb{D})$ пространство всех комплексных гармонических функций $u$, заданных в единичном круге $\mathbb{D}$, таких, что

$$
\|u\|_{\mathcal{P} M(\mathbb{D})} \stackrel{\text { def }}{=} \sup _{0 \leqslant r<1} \int_{\mathbb{T}}|u(r \zeta)||d \zeta|<+\infty .
$$


Хорошо известно (см., например, [31; гл. 3], что пространство $\mathcal{P} M(\mathbb{D})$ совпадает с множеством всех функций $u$, представимых в виде

$$
u(z)=(\mathcal{P} \nu)(z) \stackrel{\text { def }}{=} \frac{1}{2 \pi} \int_{\mathbb{T}} \frac{\left(1-|z|^{2}\right) d \nu(\zeta)}{|z-\zeta|^{2}}, \quad z \in \mathbb{D},
$$

где $\nu$ - комплексная борелевская мера на $\mathbb{T}$, при этом $\|u\|_{\mathcal{P} M(\mathbb{D})}=\|\nu\|_{M(\mathbb{T})} \stackrel{\text { def }}{=}$ $|\mu|(\mathbb{T})$.

Обозначим через через $\mathcal{P} L(\mathbb{D})$ подпространство пространства $\mathcal{P} M(\mathbb{D})$, состоящее из функций $u \in \mathcal{P} L(\mathbb{D})$, которым соответствует абсолютно непрерывная мера $\nu$.

Положительную меру $\mu$ на $\mathbb{D}$ будем называть мерой Карлесона в сильном смысле, если $\int_{\mathbb{D}}|u(z)| d \mu(z)<\infty$ для любой функции $u \in \mathcal{P} M(\mathbb{D})$. Заметим, что пространство $\mathcal{P} M(\mathbb{D})$ содержит класс Харди $H^{1}$ в единичном круге $\mathbb{D}$. Отсюда следует, что любая мера Карлесона в сильном смысле является мерой Карлесона в обычном смысле. Обозначим символом $\mathrm{CM}_{\mathrm{S}}(\mathbb{D})$ пространство всех мер Радона $\mu$ в $\mathbb{D}$ таких, что $|\mu|$ - мера Карлесона в сильном смысле. Положим

$$
\|\mu\|_{\mathrm{CM}_{\mathrm{s}}(\mathbb{D})} \stackrel{\text { def }}{=} \sup \left\{\int_{\mathbb{D}}|u(z)| d \mu(z): u \in \mathcal{P} M(\mathbb{D}),\|u\|_{\mathcal{P} M(\mathbb{D})} \leqslant 1\right\} .
$$

Легко видеть, что

$$
\|\mu\|_{\mathrm{CM}_{\mathrm{s}}(\mathbb{D})}=\sup \left\{\int_{\mathbb{D}}|u(z)| d \mu(z): u \in \mathcal{P} L(\mathbb{D}),\|u\|_{\mathcal{P} M(\mathbb{D})} \leqslant 1\right\}
$$

и

$$
\|\mu\|_{\mathrm{CM}_{\mathrm{s}}(\mathbb{D})}=\frac{1}{2 \pi} \sup _{\zeta \in \mathbb{T}} \int_{\mathbb{D}} \frac{\left(1-|w|^{2}\right) d \mu(w)}{|\zeta-w|^{2}}
$$

Заметим, что

$$
\sup _{\zeta \in \mathbb{T}} \int_{\mathbb{D}} \frac{\left(1-|w|^{2}\right) d \mu(w)}{|\zeta-w|^{2}}=\sup _{z \in \mathbb{D}} \int_{\mathbb{D}} \frac{\left(1-|w|^{2}\right) d \mu(w)}{|1-z \bar{w}|^{2}} .
$$

Это вытекает из принципа максимума для $L^{2}$-значных аналитических функций в круге $\mathbb{D}$.

Сформулируем теперь в наших обозначениях достаточное условие АразиБартона-Фридмана в случае окружности (см. [15]).

Теорема 3.13.7. Пусть $f$ - функиия, аналитическая в единичном круге $\mathbb{D}$. Предположим, что $\zeta^{-1} f^{\prime}(\zeta) d \boldsymbol{m}_{2}(\zeta) \in \mathrm{CM}_{\mathrm{s}}(\mathbb{D})$. Тогда функиия $f$ имеет конечные граничные значения всюоу на окружности $\mathbb{T}$ (которые мы обозначаем той же буквой $f), f \in(\mathrm{CL})^{\prime}(\mathbb{D}) u$

$$
\|f-f(0)\|_{(\mathrm{CL})^{\prime}(\mathbb{D})} \leqslant 2\left\|\zeta^{-1} f^{\prime}(\zeta) d \boldsymbol{m}_{2}(\zeta)\right\|_{\mathrm{CM}_{\mathrm{s}}(\mathbb{D})} .
$$


Нам понадобится аналог леммы 3.13.2.

Лемма 3.13.8. Пусть $f$ - аналитическая в единичном круге $\mathbb{D}$ функиия. Предположим, что $\int_{\mathbb{D}}\left(1-|w|^{2}\right)\left|f^{\prime}(w)\right| d \boldsymbol{m}_{2}(w)<+\infty$. Тогда при всех $z \in \mathbb{C}_{+}$ $f(z)-f(0)=\frac{1}{\pi} \int_{\mathbb{D}} \frac{\left(1-|w|^{2}\right) f^{\prime}(w) d \boldsymbol{m}_{2}(w)}{(1-z \bar{w})^{2} \bar{w}}=\frac{1}{\pi} \int_{\mathbb{C} \backslash \mathbb{D}} \frac{\left(|w|^{2}-1\right) f^{\prime}\left(\bar{w}^{-1}\right) d \boldsymbol{m}_{2}(w)}{(w-z)^{2} \bar{w}^{3}}$.

ДокАЗАТЕльство. Мы докажем только первое равенство, поскольку второе равенство получается из первого при помощи замены переменной $w \mapsto \bar{w}^{-1}$. При $z=0$ доказываемое равенство вытекает из теоремы о среднем. Остаётся заметить, что

$$
f^{\prime}(z)=\frac{2}{\pi} \int_{\mathbb{D}} \frac{\left(1-|w|^{2}\right) f^{\prime}(w) d \boldsymbol{m}_{2}(w)}{(1-z \bar{w})^{3}}
$$

для всех $z \in \mathbb{D}$ (см., например, [30; следствие 1.5]).

Отметим ещё, что первое равенство этой леммы может быть получено дифференцированием по $z$ равенства (4.3) статьи [15].

ДОКАЗАТЕЛЬСТВО ТЕОРЕМЫ 3.13.7. ПоЛоЖим

$$
\begin{aligned}
F(z) & \stackrel{\text { def }}{=} \frac{1}{\pi} \int_{\mathbb{D}} \frac{\left(1-|w|^{2}\right) f^{\prime}(w)}{\bar{w}^{2}}\left(\frac{1}{1-z \bar{w}}-1\right) d \boldsymbol{m}_{2}(w) \\
& =\frac{z}{\pi} \int_{\mathbb{D}} \frac{\left(1-|w|^{2}\right) f^{\prime}(w) d \boldsymbol{m}_{2}(w)}{\bar{w}(1-z \bar{w})}
\end{aligned}
$$

для всех $z$ из $\mathbb{C}$ таких, что $|z| \leqslant 1$. Сходимость интегралов при $z \in \mathbb{T}$ вытекает из неравенства Коши-Буняковского, если принять во внимание тождество (3.13.3). Заметим, что $F^{\prime}(z)=\frac{1}{\pi} \int_{\mathbb{D}} \frac{\left(1-|w|^{2}\right) f^{\prime}(w) d \boldsymbol{m}_{2}(w)}{(1-z \bar{w})^{2} \bar{w}}=f(z)-f(0)$ в силу леммы 3.13.2. Рассмотрим меру Радона $\mu$ в $\mathbb{C} \backslash \overline{\mathbb{D}}$ :

$$
d \mu(w) \stackrel{\text { def }}{=} \frac{1}{\pi} \bar{w}^{-3}\left(|w|^{2}-1\right) f^{\prime}\left(\bar{w}^{-1}\right) d \boldsymbol{m}_{2}(w) .
$$

Тогда

$$
\begin{aligned}
\widehat{\mu}_{0}(z) & =\frac{1}{\pi} \int_{\mathbb{C} \backslash \mathbb{D}} \frac{\left(|w|^{2}-1\right) f^{\prime}\left(\bar{w}^{-1}\right)}{\bar{w}^{3}}\left(\frac{1}{w-z}-\frac{1}{w}\right) d \boldsymbol{m}_{2}(w) \\
& =\frac{1}{\pi} \int_{\mathbb{D}} \frac{\left(1-|w|^{2}\right) f^{\prime}(w)}{\bar{w}^{2}}\left(\frac{1}{1-z \bar{w}}-1\right) d \boldsymbol{m}_{2}(w)=F(z) .
\end{aligned}
$$


Заметим, что

$$
\begin{aligned}
\|\mu\|_{\mathscr{M}(\mathbb{C} \backslash \operatorname{clos} \mathbb{D})} & =\sup _{z \in \operatorname{clos} \mathbb{D}} \int_{\mathbb{C} \backslash \operatorname{clos} \mathbb{D}} \frac{d|\mu|(w)}{|w-z|^{2}} \\
& =\frac{1}{\pi} \sup _{z \in \mathbb{D}} \int_{\mathbb{C} \backslash \operatorname{clos} \mathbb{D}} \frac{\left(|w|^{2}-1\right)\left|f^{\prime}\left(\bar{w}^{-1}\right)\right|}{|w-z|^{2}|w|^{3}} d \boldsymbol{m}_{2}(w) \\
& =\frac{1}{\pi} \sup _{z \in \operatorname{clos} \mathbb{D}} \int_{\mathbb{D}} \frac{\left(1-|w|^{2}\right)\left|f^{\prime}(w)\right|}{|1-z \bar{w}|^{2}|w|} d \boldsymbol{m}_{2}(w) \\
& =\frac{1}{\pi} \sup _{\zeta \in \mathbb{T}} \int_{\mathbb{D}} \frac{\left(1-|w|^{2}\right)\left|f^{\prime}(w)\right|}{|\zeta-w|^{2}|w|} d \boldsymbol{m}_{2}(w)=2\left\|\zeta^{-1} f^{\prime}(\zeta) d \boldsymbol{m}_{2}(\zeta)\right\|_{\mathrm{CM}_{\mathbb{s}}(\mathbb{D})} .
\end{aligned}
$$

Теперь из теоремы 3.8.1 следует, что

$$
\|f-f(0)\|_{(\mathrm{CL})^{\prime}(\mathbb{D})}=\|F\|_{\mathrm{CL}(\mathbb{D})} \leqslant\|\mu\|_{\mathscr{M}(\mathbb{C} \backslash \operatorname{clos} \mathbb{D})}=2\left\|\zeta^{-1} f^{\prime}(\zeta) d \boldsymbol{m}_{2}(\zeta)\right\|_{\mathrm{CM}_{\mathrm{s}}(\mathbb{D})} .
$$

Теорема доказана.

СЛЕДСТВИЕ 3.13.9. Пусть $f$ - функиия, аналитическая в единичном круге $\mathbb{D}$. Предположим, что $f^{\prime} d \boldsymbol{m}_{2} \in \mathrm{CM}_{\mathrm{S}}(\mathbb{D})$. Тогда функиия $f$ имеет конечные граничные значения всюду на окружности $\mathbb{T}$ (которые мы обозначаем той же буквой $f), f \in(\mathrm{CL})^{\prime}(\mathbb{D}) u\|f-f(0)\|_{(\mathrm{CL})^{\prime}(\mathbb{D})} \leqslant$ const $\left\|f^{\prime} d \boldsymbol{m}_{2}\right\|_{\mathrm{CM}_{\mathrm{s}}(\mathbb{D})}$.

ДокАзАтельство. Достаточно заметить, что для любой непрерывной в $\mathbb{D}$ функции $h$ условие $h d \boldsymbol{m}_{2} \in \mathrm{CM}_{\mathrm{s}}(\mathbb{D})$ влечёт, что $\zeta^{-1} h(\zeta) d \boldsymbol{m}_{2}(\zeta) \in \mathrm{CM}_{\mathrm{s}}(\mathbb{D})$. Остаётся сослаться на теорему о замкнутом графике. Следствие доказано.

ЗАмЕЧАНИЕ. Можно отказаться от использования теоремы о замкнутом графике и получить следующую явную оценку:

$$
C_{s}\left(|\zeta|^{-1} h(\zeta) d \boldsymbol{m}_{2}(\zeta)\right) \leqslant \frac{8}{3} C_{s}\left(h d \boldsymbol{m}_{2}\right)
$$

для любой субгармонической в $\mathbb{D}$ функции $h$, но нам это не понадобится.

Tеорема 3.13.10. Если $\|\nabla u\| d \boldsymbol{m}_{2} \in \mathrm{CM}_{\mathrm{S}}(\mathbb{D})$ для некоторой гармонической в $\mathbb{D}$ функции $и$, то и имеет граничные значения всюду на $\mathbb{T} u u \in(\mathrm{OL})_{\mathrm{loc}}^{\prime}(\mathbb{T})$.

ДокАЗАтельство. Функцию $u$ можно представить в виде $u=f+\bar{g}$, где $f$ и $g$ - аналитические в $\mathbb{D}$ функции. Из следствия 3.13 .9 вытекает, что $f, g \in$ $(\mathrm{OL})^{\prime}(\mathbb{T})$. Остаётся заметить, что из определения пространства $(\mathrm{OL})_{\text {loc }}^{\prime}(\mathbb{T})$ мгновенно вытекает его инвариантность относительно комплексного сопряжения. Теорема доказана.

СлЕДСтвие 3.13.11. Если $\|$ Hess $u \| d \boldsymbol{m}_{2} \in \mathrm{CM}_{\mathrm{s}}(\mathbb{D})$ для некоторой гармонической в $\mathbb{D}$ функции $и$, то и продолжается до непрерьвной функции на $\mathbb{D} \cup \mathbb{T}$ $u u \in \mathrm{OL}(\mathbb{T})$.

Отсюда легко вытекает следующий результат работы [56], доказательство которого приведено в разделе 1.6 настоящего обзора (см. теорему 1.6.2).

Teорема 3.13.12. Пусть $f \in B_{\infty, 1}^{1}(\mathbb{T})$. Тогдa $f \in \mathrm{OL}(\mathbb{T})$. 
Мы вывели достаточное условие Арази-Бартона-Фридмана из теоремы 3.8.1. Можно показать, что теорема 3.8.1 даёт примеры операторно липшицевых функций, которые не удовлетворяют аналогу достаточного условия Арази-Бартона-Фридмана для $\mathbb{C}_{+}$. В [3] построен пример функции $f \in \widehat{\mathscr{M}}\left(\operatorname{clos} \mathbb{C}_{+}\right)$такой, что $f^{\prime \prime} d \boldsymbol{m}_{2} \notin \mathrm{CM}_{\mathrm{S}}\left(\mathbb{C}_{+}\right)$. Из теоремы 3.8.1 следует, что такая функция $f$ принадлежит пространству $(\mathrm{CL})^{\prime}\left(\mathbb{C}_{+}\right)$, хотя достаточное условие Арази-Бартона-Фридмана к этой функции не применимо. Аналогичное утверждение справедливо и для функций в $\mathbb{D}$.

ЗАмечАниЕ. В работе Арази-Бартона-Фридмана [15] было отмечено, что их достаточное условие операторной липшицевости функции, заданной на единичной окружности, влечёт достаточное условие, полученное в работе [56] (см. также раздел 1.6 настоящего обзора). Из результатов статьи [3] следует, что достаточное условие операторной липшицевости Арази-Бартона-Фридмана может работать и в том случае, когда функция $f^{\prime}$ не является непрерывной. С другой стороны, легко видеть, что если $f \in B_{\infty, 1}^{1}(\mathbb{T})$, то $f^{\prime} \in C(\mathbb{T})$. То же самое можно сказать и по поводу функций класса $B_{\infty, 1}^{1}(\mathbb{R})$ (см. теорему 1.6.4). Действительно, нетрудно проверить, что функция $f(z)=\exp \left(-\mathrm{i} z^{-1}\right)$ удовлетворяет условиям теоремы 3.13.1, хотя её сужение на вещественную прямую терпит разрыв в нуле. Отметим ещё, что в работе [3] по существу доказано, что подмножество вещественной прямой может быть множеством всех точек разрыва функции $f \mid \mathbb{R}$, где $f$ удовлетворяет условиям теоремы 3.13 .1 , в том и только том случае, когда оно принадлежит к типу $F_{\sigma}$ и не имеет внутренних точек. Можно сделать аналогичное замечание и для функций на $\mathbb{T}$ и $\mathbb{D}$.

Интересно сравнить достаточное условие операторной липшицевости, приведённое в этом разделе, с необходимым условием, приведённым в разделе 1.5. Комбинация этих условий дана в следующей теореме.

Tеорема 3.13.13. Если $f \in \operatorname{Lip}(\mathbb{R})$ u $\|$ Hess $\mathcal{P} f \| d \boldsymbol{m}_{2}-$ мера Карлесона $в$ сильном смысле, то $f \in \mathrm{OL}(\mathbb{R})$. Если же $f \in \mathrm{OL}(\mathbb{R})$, mо $\|\operatorname{Hess} \mathcal{P} f\| d \boldsymbol{m}_{2}-$ мера Карлесона.

Аналогичное утверждение справедливо и для функций на окружности $\mathbb{T}$.

\subsection{4. В каких случаях имеет место равенство $\mathrm{OL}(\mathfrak{F})=\operatorname{Lip}(\mathfrak{F})$ ?}

Tеорема 3.14.1. Предположим, что $\mathrm{OL}(\mathfrak{F})=\operatorname{Lip}(\mathfrak{F})$ для некоторого замкнутого подмножества $\mathfrak{F}$ комплексной плоскости $\mathbb{C}$. Тогда множество $\mathfrak{F}$ конечно.

ДокАЗАТЕльство. Предположим, что множество $\mathfrak{F}$ бесконечно. Тогда множество $\mathfrak{F}$ содержит предельную точку $a \in \widehat{\mathbb{C}} \stackrel{\text { def }}{=} \mathbb{C} \cup\{\infty\}$. Если $a \in \mathbb{C}$, то можно считать, что $a=0$. Случай $a=\infty$ может быть рассмотрен аналогично. Кроме того, случай $a=\infty$ сводится к случаю $a=0$ при помощи дробно-линейных преобразований.

Предположим сначала, что $\mathfrak{F} \subset \mathbb{R}$. Тогда легко построить функцию $f \in$ $\operatorname{Lip}(\mathfrak{F})$, у которой нет производной в нуле. Ясно, что $f \notin \mathrm{OL}(\mathfrak{F})$.

Чтобы отказаться от предположения $\mathfrak{F} \subset \mathbb{R}$, нам понадобится следующая лемма. 
Лемма 3.14.2. Пусть $0<q<1 u\left\{a_{n}\right\}_{n \geqslant 1}$ - последовательность положительных чисел таких, что $a_{n+1} \leqslant q a_{n}$ при всех $n \geqslant 1$. Тогда для любой числовой последовательности $b_{n}$, удовлетворяющей условию $\sum_{n \geqslant 1}\left|b_{n}\right| a_{n}^{-1}<+\infty$, найдётся функиия $v \in \mathrm{OL}(\mathbb{R})$ такая, что $v\left(a_{n}\right)=b_{n}$ при всех $n \geqslant 1$.

ДокАЗАТЕЛЬство. Зафиксируем функцию $\varphi$ класса $C^{\infty}(\mathbb{R})$ такую, что $\varphi(0)=1$ и $\operatorname{supp} \varphi \subset[-\delta, \delta]$, где число $\delta$ будет выбрано в конце доказательства. Положим $v(t) \stackrel{\text { def }}{=} \sum_{n \geqslant 1} b_{n} \varphi\left(a_{n}^{-1}\left(t-a_{n}\right)\right)$. Тогда

$$
\|v\|_{\mathrm{OL}(\mathbb{R})} \leqslant \sum_{n \geqslant 1}\left|b_{n}\right| \cdot\left\|\varphi\left(a_{n}^{-1}\left(t-a_{n}\right)\right)\right\|_{\mathrm{OL}(\mathbb{R})}=\|\varphi\|_{\mathrm{OL}(\mathbb{R})} \sum_{n \geqslant 1}\left|b_{n}\right| a_{n}^{-1}<+\infty
$$

и $v\left(a_{n}\right)=b_{n}$ при всех $n \geqslant 1$, если только число $\delta$ достаточно мало. Лемма доказана.

Продолжим доказательство теоремы 3.14.1. Хорошо известно, что любая липшицева функция, заданная на подмножестве комплексной плоскости $\mathbb{C}$, продолжается до липшицевой функции, заданной на всей комплексной плоскости $\mathbb{C}$ (см., например, [70; гл. VI, §2, теорема 3]). Таким образом, достаточно ограничиться случаем, когда множество $\mathfrak{F} \backslash\{0\}$ состоит из членов последовательности $\left\{\lambda_{n}\right\}_{n \geqslant 1}$, сколь угодно быстро стремящейся к нулю. Пусть $\lambda_{n}=a_{n}+\mathrm{i} b_{n}$. Можно считать, что $\lim _{n \rightarrow \infty} \lambda_{n} /\left|\lambda_{n}\right|=1$ и вещественные последовательности $\left\{a_{n}\right\}_{n \geqslant 1}$ и $\left\{b_{n}\right\}_{n \geqslant 1}$ удовлетворяют условиям леммы 3.14.2. Положим $h(t) \stackrel{\text { def }}{=} t+\mathrm{i} v(t)$, где $v$ обозначает то же, что в лемме 3.14.2. Теперь случай множества $\mathfrak{F}$ сводится к уже разобранному случаю множества $\operatorname{Re} \mathfrak{F}$, поскольку

$$
\|A-B\| \leqslant\|h(A)-h(B)\| \leqslant\left(1+\|v\|_{\mathrm{OL}(\mathbb{R})}\right)\|A-B\|
$$

для любых самосопряжённых операторов $A$ и $B$ таких, что $\sigma(A), \sigma(B) \subset \operatorname{Re} \mathfrak{F}$. Теорема доказана.

\section{Заключительные замечания}

В этом разделе мы вкратце коснёмся некоторых результатов, которые не вошли в основную часть статьи.

1. Операторные модули непрерывности. Для непрерывной функции $f$ на $\mathbb{R}$ операторный модуль непрерывности $\Omega_{f}$ определяется равенством

$\Omega_{f}(\delta) \stackrel{\text { def }}{=} \sup \{\|f(A)-f(B)\|: A, B$ - самосопряжённые операторы, $\|A-B\|<\delta\}$.

Операторные модули непрерывности были введены в [8] и подробно изучались в [11]. Теорема 1.7.3, сформулированная в настоящей статье, означает, что если $f \in \Lambda_{\omega}(\mathbb{R})$, где $\omega$ - модуль непрерывности, то

$$
\Omega_{f}(\delta) \leqslant \operatorname{const} \omega_{*}(\delta), \quad \text { где } \quad \omega_{*}(\delta) \stackrel{\text { def }}{=} \delta \int_{\delta}^{\infty} \frac{\omega(t)}{t^{2}} d t .
$$


В работе [11] обсуждается точность таких оценок и получены значительно более точные оценки для непрерывных "кусочно выпукло-вогнутых" функций $f$. В частности, получена следующая неулучшаемая оценка:

$$
\||A|-|B|\| \leqslant C\|A-B\| \log \left(2+\log \frac{\|A\|+\|B\|}{\|A-B\|}\right)
$$

для ограниченных самосопряжённых операторов $A$ и $B$. Это неравенство значительно улучшает оценку Като, полученную в работе [34].

2. Коммутаторные оценки для функций нормальных операторов. Лемма 3.7.3 позволяет получить следующую квазикоммутаторную оценку:

$$
\left\|f\left(N_{1}\right) R-R f\left(N_{2}\right)\right\| \leqslant \operatorname{const} \omega_{*}\left(\max \left\{\left\|N_{1} R-R N_{2}\right\|,\left\|N_{1}^{*} R-R N_{2}^{*}\right\|\right\}\right)
$$

для любого модуля непрерывности $\omega$, для любой функции $f$ класса $\Lambda_{\omega}(\mathbb{R})$, для любого линейного оператора $R$ нормы 1 и для любых нормальных операторов $N_{1}$ и $N_{2}$ (см. [14]). В работе [13] норма квазикоммутатора в левой части неравенства оценивается только через норму $\left\|N_{1} R-R N_{2}\right\|$. Правда, при этом нужно заменить в правой части неравенства $\omega_{*}$ на $\omega_{* *} \stackrel{\text { def }}{=}\left(\omega_{*}\right)_{*}$ :

$$
\left\|f\left(N_{1}\right) R-R f\left(N_{2}\right)\right\| \leqslant \operatorname{const} \omega_{* *}\left(\left\{\left\|N_{1} R-R N_{2}\right\|\right\}\right) .
$$

Отметим, что в случае классов Гёльдера, т.е. $\omega(t)=t^{\alpha}, 0<\alpha<1$, имеет место неравенство $\omega_{* *}(t) \leqslant \operatorname{const}(1-\alpha)^{-2} t^{\alpha}$. Иными словами, мы получаем коммутаторно гёльдерову оценку.

\section{3. Подход Никольской-Фарфоровской к операторно гёльдеровым} функциям. В работе [49] предлагается альтернативный подход к операторно гёльдеровым функциям. Он основан на следующем утверждении.

Пусть $0<\alpha<1$. Тогда $\Lambda_{\alpha}(\mathbb{Z}) \subset \mathrm{OL}(\mathbb{Z})$. При этом существует число $c_{\alpha}$ maкое, ито $\|f\|_{\mathrm{OL}(\mathbb{Z})} \leqslant c_{\alpha}\|f\|_{\Lambda_{\alpha}(\mathbb{Z})}$.

Из этого утверждения нетрудно вывести теорему 1.7.2, если воспользоваться легко проверяемым неравенством $\Omega_{f}(\delta) \leqslant 2 \omega_{f}(\delta / 2)+2\|f(\delta x)\|_{\mathrm{OL}(\mathbb{Z})}$. Само это утверждение можно доказать посредством интерполирования функции класса $\Lambda_{\alpha}(\mathbb{Z})$ функцией класса $B_{\infty, 1}^{1}(\mathbb{R})$ и применения теоремы 1.6 .1 , хотя в работе [49] это было доказано совсем другим способом.

4. Функции наборов коммутирующих самосопряжённых операторов. Изучение функций от нормальных операторов эквивалентно изучению функций от пар коммутирующих самосопряжённых операторов. В работе [48] удалось распространить результаты работы [14] (см. раздел 3.7 настоящего обзора) на случай функций от произвольного числа коммутирующих самосопряжённых операторов. При этом использовались совсем другие методы.

В работе [4] получены обобщения на операторно липшицевы функции от $n$ переменных некоторых результатов работы [2] о дробно-линейных подстановках (см. раздел 3.10 настоящего обзора). В многомерной ситуации роль дробно-линейных преобразований играют преобразования Мёбиуса, т. е. суперпозиции конечного числа инверсий. 
5. Липшицевы функции наборов коммутирующих самосопряжённых операторов. В работе [35] результаты работы [67] обобщаются на случай функций наборов $n$ коммутирующих самосопряжённых операторов и получается липшицева оценка в норме $\boldsymbol{S}_{p}, 1<p<\infty$, для липшицевых функций в $\mathbb{R}^{n}$.

\section{6. Функции пар некоммутирующих самосопряжённых операторов.}

Для пары $(A, B)$ не обязательно коммутирующих самосопряжённых операторов в работе [6] рассматривались функции $f(A, B)$, которые определяются с помощью двойных операторных интегралов, и изучалось поведение таких функций при возмущении пары. Оказалось, что, в отличие от функций коммутирующих операторов, липшицевы оценки в операторной норме и в ядерной норме сильно отличаются друг от друга. В частности, в работе [6] показано, что для $f \in B_{\infty, 1}^{1}\left(\mathbb{R}^{2}\right)$ имеет место неравенство

$$
\left\|f\left(A_{1}, B_{1}\right)-f\left(A_{2}, B_{2}\right)\right\|_{S_{p}} \leqslant \text { const }\|f\|_{B_{\infty, 1}^{1}} \max \left\{\left\|A_{1}-A_{2}\right\|_{S_{p}},\left\|B_{1}-B_{2}\right\|_{S_{p}}\right\}
$$

при $p \in[1,2]$. Такое неравенство было получено ранее в [14] для функций коммутирующих операторов при $p \geqslant 1$. Однако в случае функций некоммутирующих операторов это неравенство для $p \geqslant 2 u$ для операторной нормы неверно (см. [6]).

В качестве инструмента в [6] использовались тройные операторные интегралы и были введены модифицированные хогеруповы тензорные произведения пространств $L^{\infty}$.

7. Операторно липшицевы функции и формула следов Лифшица-Крейна. Пусть $A$ и $B$ - самосопряжённые операторы с ядерной разностью $A-B$. Такой паре соответствует единственная вещественная функция $\xi$ класса $L^{1}(\mathbb{R})$, называемая функиией спектрального сдвига, такая, что для достаточно хороших функций $f$ на $\mathbb{R}$ имеет место формула следов Лифшица-Крейна

$$
\operatorname{trace}(f(A)-f(B))=\int_{\mathbb{R}} f^{\prime}(t) \xi(t) d t
$$

(см. [43] и [41]). В работе М. Г. Крейна [41] показано, что эта формула справедлива для функций $f$, производная которых является преобразованием Фурье комплексной меры. В работе [58] формула следов распространена на функции $f$ класса Бесова $B_{\infty, 1}^{1}(\mathbb{R})$. В теореме 3.6.5 настоящего обзора показано, что для того, чтобы оператор $f(A)-f(B)$ был ядерным при условии ядерности оператора $A-B$, необходимо и достаточно, чтобы функция $f$ была операторно липшицевой. Наконец, в недавней работе [64] показано, что для операторно липшицевых функций левая часть формулы следов Лифшица-Крейна не только имеет смысл, но и совпадает с её правой частью. Другими словами, формула Лифиица-Крейна справедлива для любых самосопряжённых операторов $A$ и $B$ с ядерной разностью в том и только том случае, когда функиия $f$ операторно липшицева.

В завершение упомянем недавний обзор [63], в котором рассматриваются приложения кратных операторных интегралов в различных задачах теории возмущений. 


\section{Список литературы}

[1] Н. И. Ахиезер, Лекции по теории аппроксимации, 2-е изд., Наука, М., 1965, 407 с.; нем. пер.: N. I. Achieser, Vorlesungen über Approximationstheorie, Math. Lehrbücher und Monogr., II, Akademie-Verlag, Berlin, 1967, xiii+412 pp.

[2] А. Б. Александров, "Операторно липшицевы функции и дробно-линейные преобразования", Исследования по линейным операторам и теории функций. 40, Зап. науч. сем. ПОМИ, 401, ПОМИ, СПб., 2012, 5-52; англ. пер.: А. В. Aleksandrov, "Operator Lipschitz functions and linear fractional transformations", J. Math. Sci. (N. Y.), 194:6 (2013), 603-627.

[3] А.Б. Александров, "Операторно липшицевы функции и модельные пространства", Исследования по линейным операторам и теории функиий. 41, Зап. науч. сем. ПОМИ, 416, ПОМИ, СПб., 2013, 5-58; англ. пер.: А. В. Aleksandrov, "Operator Lipschitz functions and model spaces", J. Math. Sci. (N. Y.), 202:4 (2014), 485-518.

[4] А.Б. Александров, "Операторно липшицевы функции нескольких переменных и преобразования Мёбиуса", Исследования по линейным операторам и теории функиий. 42, Зап. науч. сем. ПОМИ, 424, ПОМИ, СПб., 2014, 5-32; англ. пер.: A. B. Aleksandrov, "Operator Lipschitz functions in several variables and Möbius transformations", J. Math. Sci. (N. Y.), 209:5 (2015), 665-682.

[5] А.Б. Александров, "Коммутаторно липшицевы функции и аналитическое продолжение", Исследования по линейным операторам и теории функиий. 43, Зап. науч. сем. ПОМИ, 434, ПОМИ, СПб., 2015, 5-18.

[6] A. Aleksandrov, F. Nazarov, V. Peller, "Triple operator integrals in Schatten-von Neumann norms and functions of perturbed noncommuting operators", C. R. Math. Acad. Sci. Paris, 353:8 (2015), 723-728.

[7] A. Aleksandrov, V. Peller, "Functions of perturbed operators", C. R. Math. Acad. Sci. Paris, 347:9-10 (2009), 483-488.

[8] A. B. Aleksandrov, V.V. Peller, "Operator Hölder-Zygmund functions", Adv. Math., 224:3 (2010), 910-966.

[9] A. B. Aleksandrov, V.V. Peller, "Functions of operators under perturbations of class $\mathbf{S}_{p} "$, J. Funct. Anal., 258:11 (2010), 3675-3724.

[10] A. B. Aleksandrov, V. V. Peller, "Functions of perturbed unbounded self-adjoint operators. Operator Bernstein type inequalities", Indiana Univ. Math. J., 59:4 (2010), 1451-1490.

[11] A.B. Aleksandrov, V.V. Peller, "Estimates of operator moduli of continuity", J. Funct. Anal., 261:10 (2011), 2741-2796.

[12] А. Б. Александров, В. В. Пеллер, "Функции от возмущённых диссипативных операторов", Алгебра и анализ, 23:2 (2011), 9-51; англ. пер.: А. B. Aleksandrov, V. V. Peller, "Functions of perturbed dissipative operators", St. Petersburg Math. J., 23:2 (2012), 209-238.

[13] A. B. Aleksandrov, V. V. Peller, "Operator and commutator moduli of continuity for normal operators", Proc. Lond. Math. Soc. (3), 105:4 (2012), 821-851.

[14] A. B. Aleksandrov, V. V. Peller, D. S. Potapov, F. A. Sukochev, "Functions of normal operators under perturbations", Adv. Math., 226:6 (2011), 5216-5251.

[15] J. Arazy, T. J. Barton, Y. Friedman, "Operator differentiable functions", Integral Equations Operator Theory, 13:4 (1990), 461-487.

[16] G. Bennett, "Schur multipliers", Duke Math. J., 44:3 (1977), 603-639.

[17] S. K. Berberian, "Note on a theorem of Fuglede and Putnam", Proc. Amer. Math. Soc., 10:2 (1959), 175-182.

[18] С. Н. Бернштейн, "Распространение неравенства С. Б. Стечкина на целые функции конечной степени", Докл. АН СССР, 60:9 (1948), 1487-1490. 
[19] М.Ш. Бирман, М. З. Соломяк, "Двойные операторные интегралы Стилтьеса", Спектральная теория и волновые процессы, Пробл. матем. физ., $\mathbf{1}$, ЛГУ, Л., 1966, 33-67.

[20] М. Ш. Бирман, М. З. Соломяк, “Двойные операторные интегралы Стилтьеса. II”, Спектральная теория, проблемы дифракиии, Пробл. матем. физ., 2, ЛГУ, Л., 1967, 26-60.

[21] М.Ш. Бирман, М.З. Соломяк, “Двойные операторные интегралы Стилтьеса. III. Предельный переход под знаком интеграла", Теория функиий. Спектралъная теория. Распространение волн, Пробл. матем. физ., 6, ЛГУ, Л., 1973, 27-53.

[22] M. Birman, M. Solomyak, "Tensor product of a finite number of spectral measures is always a spectral measure", Integral Equations Operator Theory, 24:2 (1996), 179-187.

[23] Ю. Л. Далецкий, С. Г. Крейн, "Интегрирование и дифференцирование функций эрмитовых операторов и приложения к теории возмущений”, Труды семинара по функц. анализу, 1, ВГУ, Воронеж, 1956, 81-105.

[24] В.К. Дзядык, Введение в теорию равномерного приближения функиий полиномами, Наука, М., 1977, 511 с.

[25] Ю.Б. Фарфоровская, "О связи метрики Канторовича-Рубинштейна для спектральных разложений самосопряжённых операторов с функциями от операторов", Вестн. Ленингр. ун-та. Матем., мех., астроном., 1968, № 4, 94-97.

[26] Ю. Б. Фарфоровская, "Пример липшицевой функции от самосопряжённого оператора, дающей неядерное приращение при ядерном возмущении", Исследования по линейным операторам и теории функиий. III, Зап. науч. сем. ЛОМИ, 30, Изд-во "Наука", Ленинград. отд., Л., 1972, 146-153; англ. пер.: Yu. B. Farforovskaya, "Example of a Lipschitz function of self-adjoint operators that gives a nonnuclear increment under a nuclear perturbation", J. Soviet Math., 4:4 (1975), 426-433.

[27] M. Frazier, B. Jawerth, "A discrete transform and decompositions of distribution spaces", J. Funct. Anal., 93 (1990), 34-170.

[28] И.Ц. Гохберг, М.Г. Крейн, Введение в теорию линейных несамосопряжённых операторов, Наука, М., 1965, 448 с.; англ. пер.: I. C. Gohberg, M. G. Kreйn, Introduction to the theory of linear nonselfadjoint operators, Transl. Math. Monogr., 18, Amer. Math. Soc., Providence, RI, 1969, xv+378 pp.

[29] Г. Х. Харди, В.В. Рогозинский, Рядъ Фуръе, Физматгиз, М., 1962, 156 с.; пер. с англ.: G. H. Hardy, W. W. Rogosinski, Fourier series, Cambridge Tracts in Math. and Math. Phys., 38, Cambridge Univ. Press, Cambridge, 1944, 100 pp.

[30] H. Hedenmalm, B. Korenblum, K. Zhu, Theory of Bergman spaces, Grad. Texts in Math., 199, Springer-Verlag, New York, 2000, x+286 pp.

[31] К. Гофман, Банаховы пространства аналитических функций, ИЛ, М., 1963, 312 с.; пер. с англ.: K. Hoffman, Banach spaces of analytic functions, Prentice-Hall Series in Modern Analysis, Prentice-Hall, Englewood Cliffs, NJ, 1962, xiii+217 pp.

[32] B. E. Johnson, J. P. Williams, "The range of a normal derivation", Pacific J. Math., 58:1 (1975), 105-122.

[33] H. M. Kamowitz, "On operators whose spectrum lies on a circle or a line", Pacific J. Math., 20 (1967), 65-68.

[34] T. Kato, "Continuity of the map $S \mapsto|S|$ for linear operators", Proc. Japan Acad., 49:3 (1973), 157-160.

[35] E. Kissin, D. Potapov, V. Shulman, F. Sukochev, "Operator smoothness in Schatten norms for functions of several variables: Lipschitz conditions, differentiability and unbounded derivations", Proc. Lond. Math. Soc. (3), 105:4 (2012), 661-702.

[36] E. Kissin, V.S. Shulman, "On a problem of J. P. Williams", Proc. Amer. Math. Soc., 130:12 (2002), 3605-3608. 
[37] E. Kissin, V.S. Shulman, "Classes of operator-smooth functions. II. Operator-differentiable functions", Integral Equations Operator Theory, 49:2 (2004), 165-210.

[38] E. Kissin, V. S. Shulman, "Classes of operator-smooth functions. I. Operator-Lipschitz functions", Proc. Edinb. Math. Soc. (2), 48:1 (2005), 151-173.

[39] E. Kissin, V.S. Shulman, "On fully operator Lipschitz functions", J. Funct. Anal., 253:2 (2007), 711-728.

[40] F. Kittaneh, "On Lipschitz functions of normal operators", Proc. Amer. Math. Soc., 94:3 (1985), 416-418.

[41] М.Г. Крейн, "О формуле следов в теории возмущений", Матем. сб., 33(75):3 (1953), 597-626.

[42] B. Ya. Levin, Lectures on entire functions, Transl. Math. Monogr., 150, Amer. Math. Soc., Providence, RI, 1996, xvi+248 pp.

[43] И. М. Лифшиц, "Об одной задаче теории возмущений, связанной с квантовой статистикой", УМН, 7:1(47) (1952), 171-180.

[44] М. М. Маламуд, С. М. Маламуд, "Спектральная теория операторных мер в гильбертовом пространстве", Алгебра и анализ, 15:3 (2003), 1-77; англ. пер.: M. M. Malamud, S. M. Malamud, "Spectral theory of operator measures in Hilbert space", St. Petersburg Math. J., 15:3 (2004), 323-373.

[45] A. McIntosh, "Counterexample to a question on commutators", Proc. Amer. Math. Soc., 29:2 (1971), 337-340.

[46] М. А. Наймарк, "Спектральные функции симметрического оператора", Изв. АН СССР. Сер. матем., 4:3 (1940), 277-318.

[47] И.П. Натансон, Конструктивная теория функиий, Гостехиздат, М.-Л., 1949, 688 с.; нем. пер.: I. P. Natanson, Konstruktive Funktionentheorie, Akademie-Verlag, Berlin, 1955, xiv+515 pp.

[48] F. L. Nazarov, V. V. Peller, "Functions of perturbed $n$-tuples of commuting self-adjoint operators", J. Funct. Anal., 266:8 (2014), 5398-5428.

[49] Л.Н. Никольская, Ю.Б. Фарфоровская, "Операторная гёльдеровость функций Гёльдера", Алгебра и анализ, 22:4 (2010), 198-213; англ. пер.: L. N. Nikol'skaya, Yu. B. Farforovskaya, "Hölder functions are operator-Hölder", St. Petersburg Math. J., 22:4 (2011), 657-668.

[50] Н. К. Никольский, Лекиии об операторе сдвига, Наука, М., 1980, 384 с.; англ. пер.: N.K. Nikol'skii, Treatise on the shift operator. Spectral function theory, Grundlehren Math. Wiss., 273, Springer-Verlag, Berlin, 1986, xii+491 pp.

[51] N. K. Nikolski, Operators, functions, and systems: an easy reading, v. 1: Hardy, Hankel, and Toeplitz, Math. Surveys Monogr., 92, Amer. Math. Soc., Providence, RI, 2002, xiv+461 pp.

[52] J. Peetre, New thoughts on Besov spaces, Duke University Mathematics Series, 1, Mathematics Department, Duke University, Durham, NC, 1976, vi+305 pp.

[53] А.А. Пекарский, "Классы аналитических функций, определяемые наилучшими рациональными приближениями в $H_{p}$ ", Матем. сб., 127(169):1(5) (1985), 3-20; англ. пер.: A. A. Pekarskii, "Classes of analytic functions determined by best rational approximations in $H_{p}$ ", Math. USSR-Sb., 55:1 (1986), 1-18.

[54] В.В. Пеллер, "Операторы Ганкеля класса $\mathfrak{S}_{p}$ и их приложения (рациональная аппроксимация, гауссовские процессы, проблема мажорации операторов)", $M a$ тем. сб., 113(155):4(12) (1980), 538-581; англ. пер.: V. V. Peller, "Hankel operators of class $\mathfrak{S}_{p}$ and their applications (rational approximation, Gaussian processes, the problem of majorizing operators)", Math. USSR-Sb., 41:4 (1982), 443-479.

[55] В. В. Пеллер, "Описание операторов Ганкеля класса $\mathfrak{S}_{p}$ при $p>0$, исследование скорости рациональной аппроксимации и другие приложения", Матем. сб., 122(164):4(12) (1983), 481-510; англ. пер.: V. V. Peller, "A description of Hankel 
operators of class $\mathfrak{S}_{p}$ for $p>0$, an investigation of the rate of rational approximation, and other applications", Math. USSR-Sb., 50:2 (1985), 465-494.

[56] В. В. Пеллер, "Операторы Ганкеля в теории возмущений унитарных и самосопряжённых операторов", Функи. анализ и его прил., 19:2 (1985), 37-51; англ. пер.: V. V. Peller, "Hankel operators in the perturbation theory of unitary and self-adjoint operators", Funct. Anal. Appl., 19:2 (1985), 111-123.

[57] V. V. Peller, "For which $f$ does $A-B \in \mathbf{S}_{p}$ imply that $f(A)-f(B) \in \mathbf{S}_{p}$ ?", Operators in indefinite metric spaces, scattering theory and other topics (Bucharest, 1985), Oper. Theory Adv. Appl., 24, Birkhäuser, Basel, 1987, 289-294.

[58] V. V. Peller, "Hankel operators in the perturbation theory of unbounded self-adjoint operators", Analysis and partial differential equations, Lecture Notes in Pure and Appl. Math., 122, Dekker, New York, 1990, 529-544.

[59] V. V. Peller, "Functional calculus for a pair of almost commuting selfadjoint operators", J. Funct. Anal., 112:2 (1993), 325-345.

[60] В.В. Пеллер, Операторь Ганкеля и их приложения, НИЦ "Регулярная и хаотическая динамика", М.-Ижевск, 2005, 1028 с.; пер. с англ.: V. V. Peller, Hankel operators and their applications, Springer Monogr. Math., Springer-Verlag, New York, 2003, xvi+784 pp.

[61] V. V. Peller, "Multiple operator integrals and higher operator derivatives", J. Funct. Anal., 233:2 (2006), 515-544.

[62] V. V. Peller, "Differentiability of functions of contractions", Linear and complex analysis, Amer. Math. Soc. Transl. Ser. 2, 226, Amer. Math. Soc., Providence, RI, 2009, 109-131.

[63] V.V. Peller, "Multiple operator integrals in perturbation theory", Bull. Math. Sci., 6:1 (2016), 15-88.

[64] V. Peller, The Lifshits-Krein trace formula and operator Lipschitz functions, 2016, 9 pp., arXiv: 1601.00490.

[65] G. Pisier, Similarity problems and completely bounded maps, Includes the solution to "The Halmos problem", 2nd, expanded ed., Lecture Notes in Math., 1618, Springer-Verlag, Berlin, 2001, viii+198 pp.

[66] G. Pisier, "Grothendieck's theorem, past and present", Bull. Amer. Math. Soc. (N. S.), 49:2 (2012), 237-323.

[67] D. Potapov, F. Sukochev, "Operator-Lipschitz functions in Schatten-von Neumann classes", Acta Math., 207:2 (2011), 375-389.

[68] У. Рудин, Функииональный анализ, Мир, М., 1975, 443 с.; пер. с англ.: W. Rudin, Functional analysis, McGraw-Hill Series in Higher Mathematics, New York-Düsseldorf-Johannesburg, 1973, xiii+397 pp.

[69] S. Semmes, "Trace ideal criteria for Hankel operators, and applications to Besov spaces", Integral Equations Operator Theory, 7:2 (1984), 241-281.

[70] И. Стейн, Сингулярные интегралы и дифференииальные свойства функиий, Мир, M., 1973, 342 с.; пер. с англ.: Е. M. Stein, Singular integrals and differentiability properties of functions, Princeton Math. Ser., 30, Princeton Univ. Press, Princeton, NJ, 1970, xiv+290 pp.

[71] И. Стейн, Г. Вейс, Введение в гармонический анализ на евклидовых пространствах, Мир, М., 1974, 332 с.; пер. с англ.: Е. M. Stein, G. Weiss, Introduction to Fourier analysis on Euclidean spaces, Princeton Math. Ser., 32, Princeton Univ. Press, Princeton, NJ, 1971, x+297 pp.

[72] Б. Секефальви-Надь, Ч. Фояш, Гармонический анализ операторов в гилъбертовом пространстве, Мир, М., 1970, 431 с.; пер. с фр.: B. Sz.-Nagy, C. Foiaş, Analyse harmonique des opérateurs de l'espace de Hilbert, Akadémiaí Kiadó, Budapest; Masson et Cie, Paris, 1967, xi+373 pp. 
[73] А.Ф. Тиман, Теория приближений функиий действительного переменного, Физматгиз, М., 1960, 624 с.; англ. пер.: А.F. Timan, Theory of approximation of functions of a real variable, Internat. Ser. Monogr. Pure Appl. Math., 34, A Pergamon Press Book. The Macmillan Co., New York, 1963, xii+631 pp.

[74] Х. Трибель, Теория функиионалъных пространств, Мир, М., 1986, 448 с.; пер. с англ.: H. Triebel, Theory of function spaces, Monogr. Math., 78, Birkhäuser Verlag, Basel-Boston-Stuttgart, 1983, x+285 pp.

[75] H. Widom, "When are differentiable functions differentiable?", Linear and complex analysis. Problem book. 199 research problems, Lecture Notes in Math., 1043, Springer-Verlag, Berlin, 1984, 184-188.

[76] J.P. Williams, "Derivation ranges: open problems", Topics in modern operator theory (Timişoara/Herculane, 1980), Operator Theory: Adv. Appl., 2, Birkhäuser, Basel-Boston, MA, 1981, 319-328.

[77] К. Иосида, Функииональный анализ, Мир, М., 1967, 624 с.; пер. с англ.: K. Yosida, Functional analysis, Grundlehren Math. Wiss., 123, Academic Press, Inc., New York; Springer-Verlag, Berlin, 1965, xi+458 pp.

\section{Алексей Борисович Александров}

Поступила в редакцию

(Alexei B. Aleksandrov)

02.05 .2016

Санкт-Петербургское отделение

Математического института им. В. А. Стеклова

Российской академии наук

E-mail: alex@pdmi.ras.ru

\section{Владимир Всеволодович Пеллер}

(Vladimir V. Peller)

Michigan State University, East Lansing,

Michigan, USA

E-mail: peller@math.msu.edu 